\title{
THE EFFECTS OF COLOR CONCENTRATES ON THE RHEOLOGY OF TINT BASES
}

\author{
A Project Report \\ Presented to \\ the Faculty of California Polytechnic State University, \\ San Luis Obispo
}

In Partial Fulfillment

of the Requirements for the Degree of Master of Science in Polymers and Coatings Chemistry

by

Douglas James Herrick

December 2012 
(C) 2012

Douglas James Herrick

ALL RIGHTS RESERVED 


\section{COMMITTEE MEMBERSHIP}

TITLE:

AUTHOR:

DATE SUBMITTED:

COMMITTEE CHAIR:

COMMITTEE MEMBER:

COMMITTEE MEMBER
THE EFFECTS OF COLOR CONCENTRATES ON THE RHEOLOGY OF TINT BASES

Douglas James Herrick

August 24, 2012

Raymond H. Fernando, Professor

Andres W. Martinez, Assistant Professor

John P. Hagen, Professor 


\section{ABSTRACT \\ THE EFFECTS OF COLOR CONCENTRATES ON THE RHEOLOGY OF TINT BASES}

\section{Douglas James Herrick}

Waterborne coatings are formulated with a number of different ingredients; water, latex polymers, pigments, surfactants, dispersants, defoamers, biocides, coalescing aids, and rheology modifiers or thickeners. Rheology modifiers are necessary in order to improve the physical properties of the coating before, during, and after application to a substrate. There are two kinds of rheology modifiers used in waterborne coatings; associative thickeners and non-associative thickeners. Coatings formulated with associative thickeners are quite sensitive to coating variations; the slightest change in the formulation has profound effects on the rheology of the coating. The opposite is true for coatings formulated with non-associative thickeners, where the rheology of the coating is not affected by minor changes in the formulation. The rheological properties of coatings are most influenced by the latex, thickener, and surfactant components of the coating. Previous studies have shown that the most ideal balance of rheological properties come from using associative thickeners. However, when waterborne coatings with associative thickeners are tinted with colorants containing high levels of surfactants they exhibit a significant decrease in viscosity. This change in viscosity results in poor sag resistance, poor brush loading, and may also cause a reduction in tint strength of the coating.

In this work, the effect of tinting paints with six different colorants on the viscosity of the paint was studied for four different paint formulations: a pastel base and a deeptone base formulated with hydroxyethylcellulose (HEC-type) non-associative thickeners, and a pastel base and a deeptone base formulated with hydrophobically-modified, ethoxylated polyurethane (HEUR-type) associative thickeners. Gloss values and tinting strengths were obtained in accordance with ASTM methods D523-08 and D4838-88. In addition, dynamic stress and 
frequency sweeps were taken in order to study the effect of colorant addition on the viscoelastic properties of each sample. Colorant addition had little to no effects on the viscosity of the bases formulated with HEC thickeners, while there was a dramatic decrease in viscosity upon colorant addition to the bases with HEUR thickeners. Similar results were observed in the viscoelastic property analysis: little to no effects on the elastic and viscous moduli was seen with the tinted coatings formulated with the non-associative thickeners, while both the elastic and viscous moduli decreased for the coatings formulated with associative thickeners. A few of the deeptone bases showed increased moduli upon tinting compared to the parent deeptone base. The addition of colorant resulted in a decrease in tinting strength and an increase in gloss for those samples with associative thickeners, while the opposite was found for those samples with non-associative thickeners. 


\section{Acknowledgements}

I would like to thank Dr. Dane Jones for helping me out with the proper use of the color spectrophotometer, Dr. John Hagen and Dr. Andres Martinez for serving on my advisory committee and helping to make sure my presentation was clear and understandable, and most of all I would like to thank Dr. Ray Fernando for the above, providing me with funding through the Cal Poly Bill Moore Fellowship Fund, and for answering all of my questions and helping me whenever he could! 


\section{Table of Contents}

List of Tables .. $\mathbf{x}$

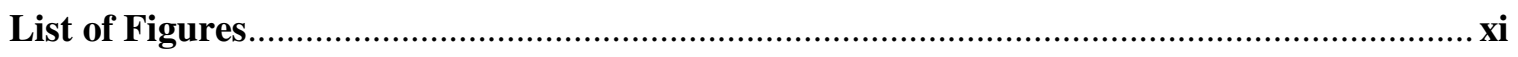

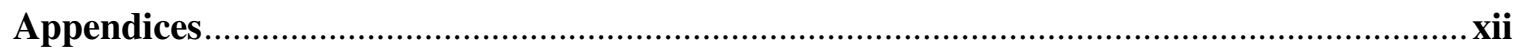

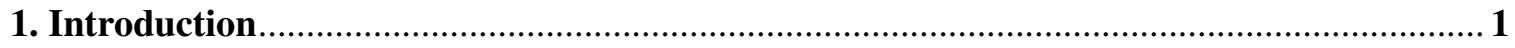

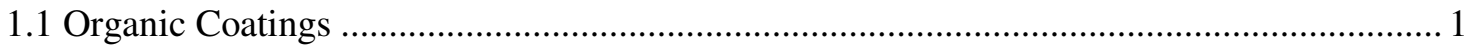

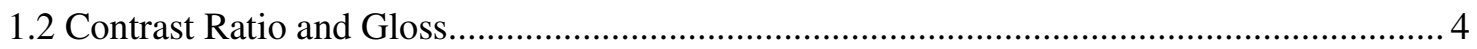

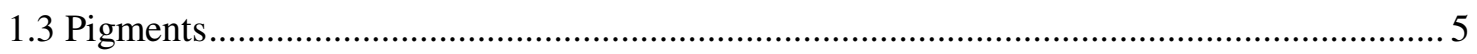

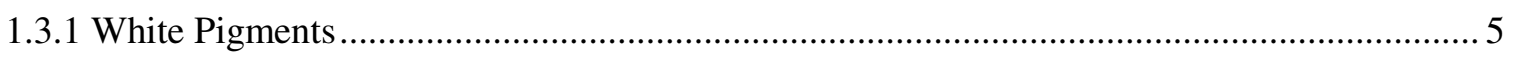

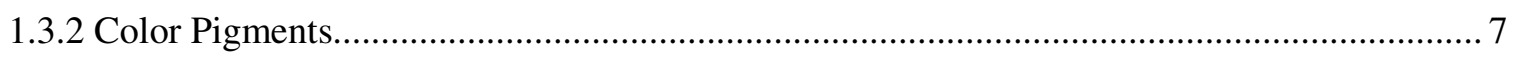

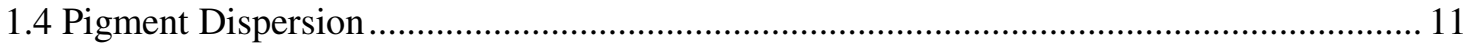

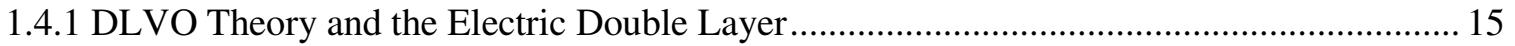

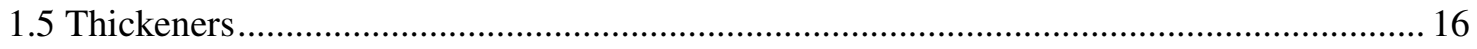

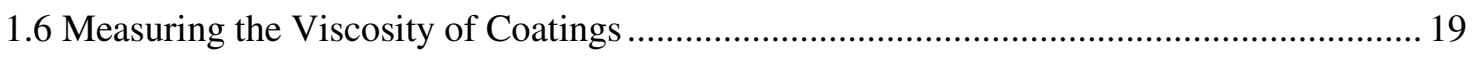

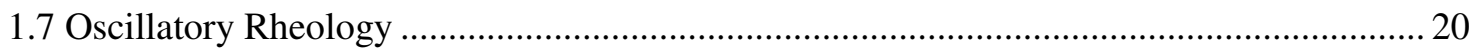

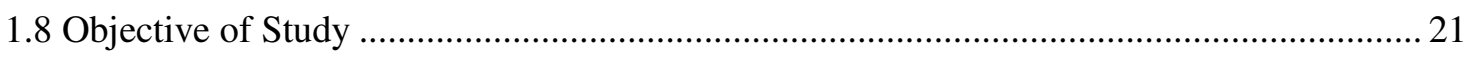

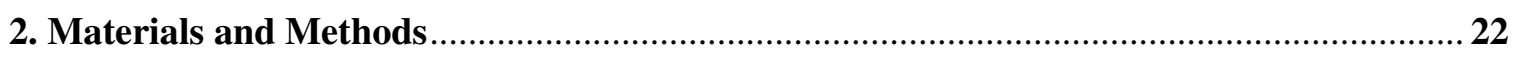

2.1 Effects of Colorant Addition on Rheology of Pastel Bases with Associative and Non-

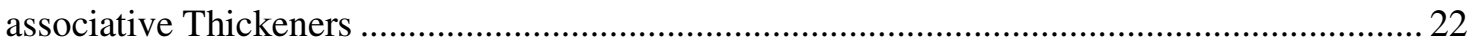

2.1.1 Pastel Base Formulation with HEC Thickener ................................................................. 22

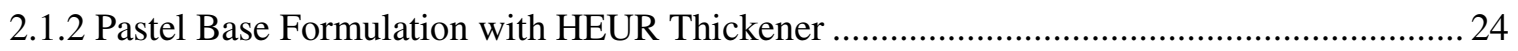

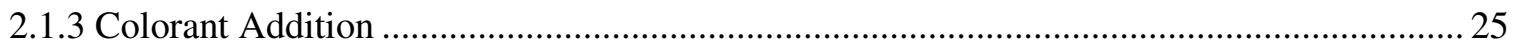

2.1.4 Viscosity Measurements and Viscoelastic Characterization............................................ 25

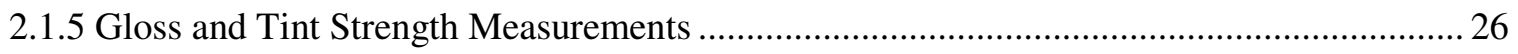

2.2 Effects of Colorant Addition on Rheology of Deeptone Bases with Associative and Non-

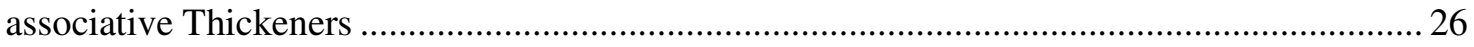

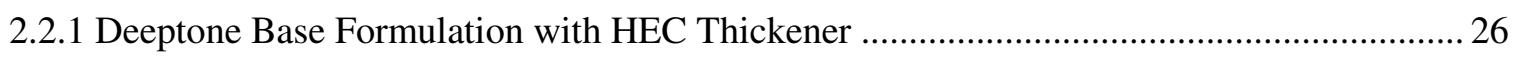

2.2.2 Deeptone Base Formulation with HEUR Thickener.......................................................... 27

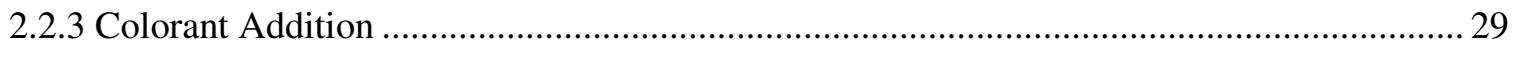

2.2.4 Viscosity Measurements and Viscoelastic Characterization..............................................29

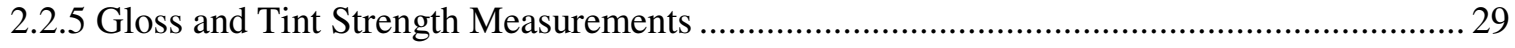

2.3 Effect of Colorant Addition on Rheology of Paints with Different Coalescing Aids.......... 30

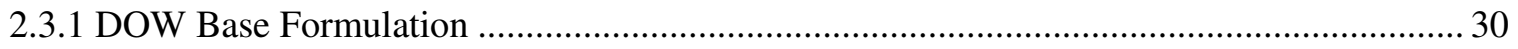




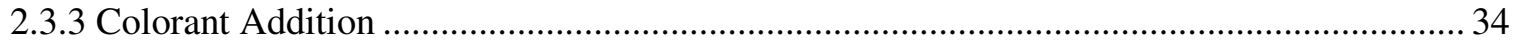

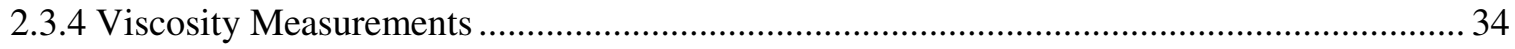

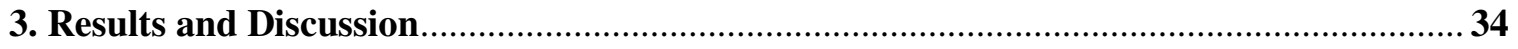

3.1 Effects of Colorant Addition on Rheology of Pastel Bases with Associative and Non-

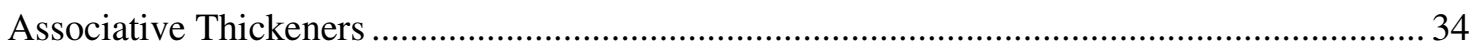

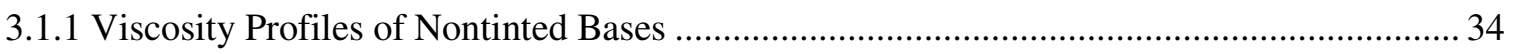

3.1.2 Viscosity Profiles of Samples Arranged by Thickener and Colorant Series......................... 35

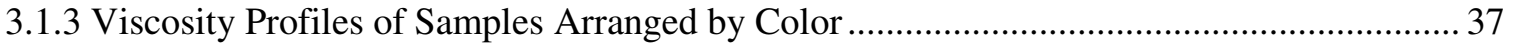

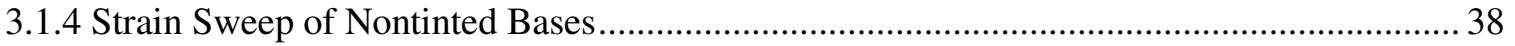

3.1.5 Strain Sweep of Samples Arranged by Thickener and Colorant Series ................................ 40

3.1.6 Strain Sweep of Samples Arranged by Thickener and Color ............................................. 41

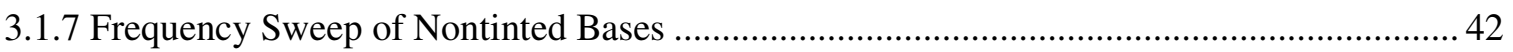

3.1.8 Frequency Sweep of Samples Arranged by Thickener and Colorant Series........................ 43

3.1.9 Frequency Sweep of Samples Arranged by Thickener and Color .......................................... 46

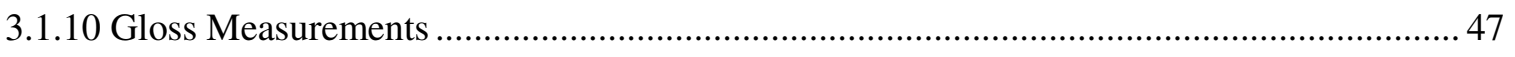

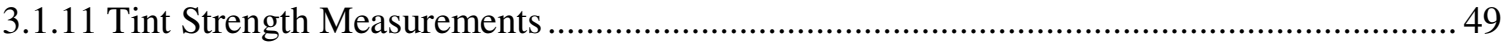

3.2 Effects of Colorant Addition on Rheology of Deeptone Bases with Associative and Non-

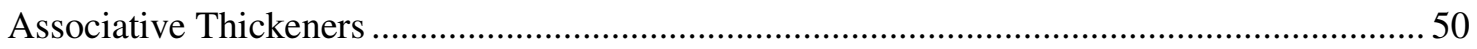

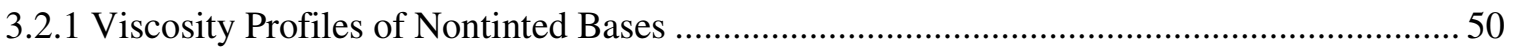

3.2.2 Viscosity Profiles of Samples Arranged by Thickener and Colorant Series......................... 51

3.2.3 Viscosity Profiles of Samples Arranged by Color ............................................................. 52

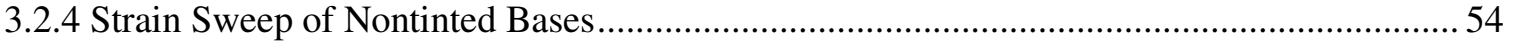

3.2.5 Strain Sweep of Samples Arranged by Thickener and Colorant Series................................ 54

3.2.6 Strain Sweep of Samples Arranged by Thickener and Color ............................................56

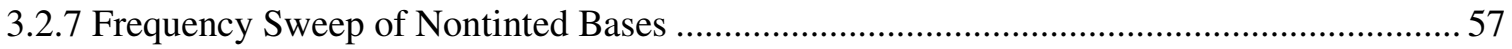

3.2.8 Frequency Sweep of Samples Arranged by Thickener and Colorant Series......................... 58

3.2.9 Frequency Sweep of Samples Arranged by Thickener and Color ....................................... 60

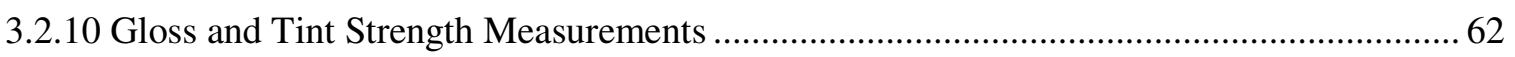

3.3 Effect of Colorant Addition on Rheology of Paints with Different Coalescing Aids.......... 63

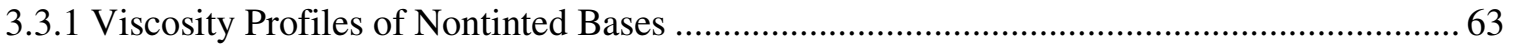

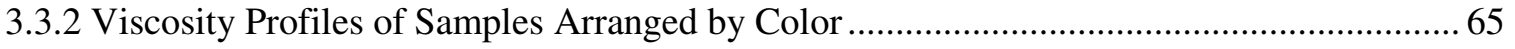

3.3.3 Viscosity Profiles of Samples Arranged by Coalescing Aid ............................................... 68 


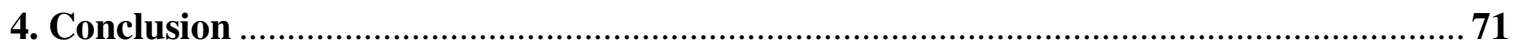

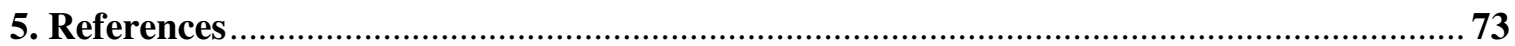




\section{LIST OF TABLES}

Table Page

1 List of Colorants and Corresponding CI Pigments Used in This Study 8

2 Pastel Base with HEC Formulation 23

3 Pastel Base with HEUR Formulation $\quad 24$

$4 \quad$ Deeptone Base with HEC Formulation 27

5 Deeptone Base with HEUR Formulation 28

6 DOW Base Formulation 31

$7 \quad$ Turk Base Formulation 33

8 Stormer Viscosities and Solids Content of Pastel Bases 35

$9 \quad$ Stormer Viscosities and Solids Content of Deeptone Bases 51 


\section{LIST OF FIGURES}

Figure Page

1 Structure of Pigment Red $122 \quad 8$

$2 \quad$ Possible Structures of Pigment Green 7 and Pigment Blue 15:2 9

$3 \quad$ Chemical Structure of Pigment Yellow $74 \quad 10$

$4 \quad$ Structures of coalescing aids used in this study 32

5 Viscosity Profiles of Pastel Bases $\quad 35$

6 Viscosity Profiles of Pastel Samples by Thickener and Colorant Series 36

$7 \quad$ Viscosity Profiles of Pastel Samples by Color $\quad 37$

$8 \quad$ Strain Sweep of Pastel Bases $\quad 39$

9 Strain Sweep of Pastel Samples Arranged by Thickener and Colorant Series $\quad 40-41$

10 Strain Sweep of Pastel Samples Arranged by Color 42

$11 \quad$ Frequency Sweep of Pastel Bases $\quad 43$

12 Frequency Sweep of Pastel Samples Arranged by Thickener and Colorant 44-45

Series

$14 \quad 60^{\circ}$ Gloss of Pastel Samples $\quad 48$

$1585^{\circ}$ Gloss of Pastel Samples $\quad 49$

16 Tint Strength of Pastel Samples $\quad 50$

17 Viscosity Profiles of Deeptone Bases $\quad 51$

18 Viscosity Profiles of Deeptone Samples by Thickener and Colorant Series 52

19 Viscosity Profiles of Deeptone Samples by Color 53

$20 \quad$ Strain Sweep of Deeptone Bases $\quad 54$

21 Strain Sweep of Deeptone Samples Arranged by Thickener and Colorant Series 55-56 
23 Frequency Sweep of Deeptone Bases

24 Frequency Sweep of Deeptone Samples Arranged by Thickener and Colorant Series

25 Frequency Sweep of Deeptone Samples Arranged by Color

$26 \quad 60^{\circ}$ and $85^{\circ}$ Gloss of Deeptone Samples

$62-63$

27 Viscosity Profiles of DOW and Turk Formulation Nontinted Bases

28 Stormer viscosities of DOW and Turk Bases with Coalescing Aids

30 Viscosity Profiles of DOW and Turk Formulation Samples Arranged by Color

31 Viscosity Profiles of DOW and Turk Formulation Samples Arranged by 


\section{APPENDICES}

1
Viscosity Profiles of Pastel Samples Arranged by Thickener and Colorant Series

Viscosity Profiles of Pastel Samples Arranged by Color

Strain Sweep of Pastel Samples Arranged by Thickener and Colorant Series

Strain Sweep of Pastel Samples Arranged by Thickener and Color

Frequency Sweep of Pastel Samples Arranged by Thickener and Colorant Series

90

Frequency Sweep of Pastel Samples Arranged by Thickener and Color

Colorant Series

Viscosity Profiles of Deeptone Samples Arranged by Color

Strain Sweep of Deeptone Samples Arranged by Thickener and Colorant Series

Strain Sweep of Deeptone Samples Arranged by Thickener and Color

102

Frequency Sweep of Deeptone Samples Arranged by Thickener and

108 Colorant Series

Frequency Sweep of Deeptone Samples Arranged by Thickener and Color Series

Gloss, Tint Strengths, and Contrast Ratios for Pastel Samples

Gloss and Contrast Ratios for Deeptone Samples

Viscosity Profiles of DOW and Turk Samples Arranged by Color

Viscosity Profiles of DOW and Turk Samples Arranged by Coalescing Aid 


\section{Introduction}

\subsection{Organic Coatings and Measuring Viscosity Loss upon Tinting}

Organic coatings are an important part of our day-to-day lives. Coatings are found everywhere, inside and outside of our homes (walls, refrigerator, outdoor siding, etc.), on buildings, bridges, boats, airplanes, even on electronic equipment. An organic coating is a liquid that is applied to a substrate which "dries" to produce a solid thin film intended to improve the properties of the material onto which it is applied. Architectural coatings are used to improve the aesthetics of a house, coatings are applied to metal structures like bridges in order to prevent corrosion, and anti-fouling coatings are applied to the underside of boats in order to prevent marine organisms from attaching. Organic coatings are made from many different ingredients. In broad terms, the ingredients can be arranged into four categories: binders, pigments, volatile components, and additives. Binders are organic polymers that are responsible for forming the film that adheres to the substrate. Pigments are solid particles that give color and opacity to the film. The volatile components constitute the dispersion medium; they make the coating low enough in viscosity for application. Additives are materials added in small quantities in order to modify properties of the coating. Rheology modifiers or thickeners are a type of additives that are used in coatings in order to modify the rheological properties of the coating. There are two types of rheology modifiers, associative thickeners and non-associative thickeners. Associative thickeners contain hydrophilic and hydrophobic regions. The hydrophobic regions of associative thickeners participate in inter- and intra-molecular associations with the surfaces of latex polymer particles and other solid particles (Hester, 1997). Non-associative thickeners contribute to viscosity by occupation of hydrodynamic volume. Higher molecular weight non-associative thickeners contribute to viscosity by participating in chain entanglements (Hester, 1997). They also differ in that associative thickeners are sensitive to coating formulation variables, while non-associative thickeners are not. The growing need to reduce volatile organic component (VOC) emissions is 
slowly phasing out the use of organic solvents altogether. Because of this trend, many formulators have replaced organic solvents with water as the liquid medium for the binder, pigments, and additives. These coatings are called waterborne coatings.

Waterborne coatings were virtually nonexistent before 1950. Restrictions on VOC content in coatings placed in the 1970's began the start of the gradual shift in the market toward the use of waterborne coatings over the traditional solventborne coatings (Jones, 2007). Waterborne coatings have three main advantages over solventborne coatings; they emit no harmful evaporated solvents, they are much less of a fire hazard and, for the user, there is greater ease in cleaning paint brushes, paint cans, and in cleaning up accidental spills. The general problem that formulators and researchers face with waterborne coatings is how to achieve a similar cost-performance relationship with solventborne coatings. Because water has a high heat capacity and heat of vaporization, energy requirements are high in order for waterborne coatings to evaporate (Jones, 2007). Also, water increases corrosion of storage tanks; this requires that corrosion resistant equipment be used in applying waterborne coatings, which increases capital cost (Jones, 2007). Many of the components of an organic coating behave differently in an aqueous medium vs. an organic medium. For example, special additives are required in a waterborne coating in order to lower the surface tension of the substrate and the surface tension of the water in order to wet the pigment particles - (this will be discussed in detail later). The presence of these surfactants tends to give coatings with poorer water resistance than solventborne coatings (Jones, 2007). This is not necessary with organic solvents, because they have low surface tensions. In addition, because associative thickeners are sensitive to coating formulation variables, coatings formulated with associative thickeners have different rheological properties in waterborne vs. solventborne coatings. By gaining an understanding of how these components interact differently in waterborne coatings, it will be possible to design formulations that perform at least as well as solventborne coatings. 
A typical waterborne coating is initially formulated in two steps called the grind and the letdown respectively. The grind is the process where the dry components (hiding pigments, fillers, etc.) are mixed together with some of the additives (biocide, defoamer, dispersing agents, surfactants, water, etc.) under high shear. During the letdown, the rest of the components, including the latex polymer, coalescing aids, defoamer, and the thickeners are added to the coating under low shear. The formulation containing all of these ingredients together is known as the coating base. Surfactants are added to the formulation in order to provide extra stabilization to the latex polymer particles and prevent them from aggregating, a process called flocculating (this will be described in detail later). The final step is called tinting the base and involves adding the color pigment, which usually comes in the form of a liquid called a colorant, to the base.

The colorant is formulated with a high concentration of surfactants, which are necessary in order to keep the pigment particles stabilized. When the colorant is added to bases formulated with associative thickeners, the viscosity of the base drops significantly due to the surfactant in the colorant (Saucy, 2008). The viscosity of the coating is directly related to the behavior of the coating as it is applied to a substrate. When a coating is applied to a substrate, it should form an even film and not sag or drip (from application onto vertical substrates). There exists an ASTM method D4400-99 and D4062-11 that quantify the sag and leveling properties of coatings. The sag and leveling properties of coatings are negatively impacted as a consequence of viscosity loss (Saucy, 2008). In addition, it is more difficult to obtain an appropriate coating thickness at lower coating viscosities. The consumer places a lot of judgment on the quality of the coating by the appearance, and a low coating viscosity could result in a poor in-can appearance (Saucy, 2008). 


\subsection{Contrast Ratio and Gloss}

Surface reflection, refraction, absorption, and scattering are all intrinsic properties that define the color of a coating. Sometimes light is able to reach all the way through the coating, reflect off of the substrate, and go back through the coating to the surface. These coatings are said to exhibit poor "hiding," a term used to describe the extent to which a coating can visually cover a substrate. The factors that determine the hiding power of a coating are the same that affect absorption and scattering. Surface roughness, increasing absorption and scattering (refractive index difference), large particle sizes, and colorant concentration all increase hiding. The color of the substrate also affects hiding because different colors reflect and absorb light at different wavelengths. Coatings chemists often use the term "contrast ratio" in order to numerically determine the extent of the hiding power of a coating. The contrast ratio of a coating is obtained by measuring the amount of light reflected off the surface of a coating over a black substrate (absorbing) over the light reflected off of the coating over a white substrate (reflecting).

According to the test method, if the contrast ratio is 0.98 or greater, the coating can be considered to completely hide the substrate.

Another important aspect regarding visual appearance of coatings is gloss. The gloss of a coating is the difference between the specular and diffuse reflection when irradiated with light at a specific angle of incidence (Jones, 2007). When a coating reflects light, it will be reflected in many different directions, and at different intensities in each direction. A coating that reflects a large fraction of light at the specular angle is said to have high gloss, while a coating that reflects a large fraction of light at angles other than the specular angle, is said to have low gloss. The gloss of a coating is mainly determined by the nature of the surface. All coatings have some degree of surface irregularities at the microscopic level causing reflection at angles other than the specular angle. A theoretically perfectly smooth coating would reflect light only at the specular angle. Pigment particles in the formulation can cause irregularities in the surface of a latex-based 
film as it coalesces, and so if the pigment volume concentration (PVC) is increased, the gloss of the film will decrease. The size of the pigment can also affect gloss because smaller pigment particles have higher surface areas, which can lead to more particles at the surface, reducing gloss. In this study, we are concerned with latex-based waterborne coatings, which generally have lower gloss values than solventborne coatings. One of the reasons for this is that, upon drying, latex-based coatings do not have the same ability to give a pigment-depleted thin top layer of the film as solventborne coatings do because latex-based coatings have both resin and pigment particles in the dispersed phase. Also, latex-based coatings are often formulated with watersoluble polymers and dispersants that are not soluble in the latex. An excess of surfactants can bloom to the surface of the film, reducing gloss. There is sometimes a loss in the gloss of a coating upon drying; as certain films expand, they crack and become brittle. This action serves to free the pigment particles from the binder; leaving them exposed at the surface. This process is called chalking. The most common method of gloss measurement is with the gloss meter, which is just a simplified goniophotometer that measures the reflectance of the coating at the specular angle.

\subsection{Pigments}

One of the most important factors affecting gloss is pigmentation (Jones, 2007). Pigments are finely divided, insoluble particles that are added to a coating in order to do one or more of the following: to modify the physical properties of the film, to hide the substrate, or to provide color. Pigments can actually reduce the cost of a coating through either one or a combination of the properties above. The particle size of a given pigment affects many different coating properties such as exterior durability, solvent resistance, transparency/opacity, and tint strength. The tint strength of a coating is the measure to the extent of which the base is affected by a certain quantity of colorant. One may think of tinting strength as the intensity of color imparted to a base from a certain quantity of colorant. The tint strength of a coating depends on the size and nature 
of the pigment particles used; typically organic pigments give higher tinting strength than inorganic pigments and smaller particles impart higher tinting strength than larger ones.

\subsubsection{White Pigments}

White pigments are discussed separately from color pigments because they are used in almost all coatings in addition to color pigments. The main function of white pigments in coatings is to provide hiding and opacity. The ideal white pigment would absorb no visible light, have a high scattering coefficient, and good hiding power. Because light scattering at the interface between media depends on the refractive index difference, it is a critical property of white pigments. The most commonly used white pigment for coatings is titanium dioxide $\left(\mathrm{TiO}_{2}\right)$, the first advantage being the high refractive index of the pigment. There are two main crystal structures of $\mathrm{TiO}_{2}$ : anatase and rutile. Anatase $\mathrm{TiO}_{2}$ has an average (with incident light of wavelength in the visible region) refractive index of 2.55 , absorbs no visible light, and costs less than rutile $\mathrm{TiO}_{2}$. Rutile $\mathrm{TiO}_{2}$ has an average refractive index of 2.73 and absorbs some violet light, but still gives about $20 \%$ greater hiding power than anatase. The refractive index of $\mathrm{TiO}_{2}$ also varies with the incident wavelength of light. In the visible spectrum, $\mathrm{TiO}_{2}$ has a higher refractive index at shorter wavelengths (blue region) and a lower refractive index at longer wavelengths (red region). This is what accounts for the loss in hiding power of $\mathrm{TiO}_{2}$ paints with red or yellow pigments. There are other white pigments besides titanium dioxide: lead carbonate $\left(2 \mathrm{PbCO}_{3} \cdot \mathrm{Pb}(\mathrm{OH})_{2}\right)$ was once used as a white pigment. It was discontinued in the late 1930 's when titanium dioxide became available (which provided much more hiding power) (Jones, 2007). White lead is soluble to a certain extent in water, and is toxic. Because of this, the lead content of any commercial paint sold in the U.S. must be $0.06 \%$ or lower of the dry weight. Other white pigments used are zinc oxide, zinc sulfide, and lithopone $\left(\mathrm{ZnS} / \mathrm{BaSO}_{4}\right)$. These pigments are rarely used in coatings because they cannot compete with titanium dioxide in terms of hiding (average refractive indices of 2.02, 2.37, and 2.0, vs. 2.73 for rutile $\mathrm{TiO}_{2}$ ). Many formulators take 
advantage of the cheap hiding power afforded by air bubbles trapped within the coating. The refractive index of air is 1.0, while that of a typical binder's is about 1.5. This gives a small refractive index difference of 0.5 , which is less than the difference with rutile $\mathrm{TiO}_{2}(1.23)$, but the approach is much cheaper because less hiding pigment is necessary. Including air bubbles in the coating can be achieved by formulating above the critical pigment volume concentration, which is the point at which there is just sufficient binder to provide a complete adsorbed layer on the pigment surfaces and to fill all of the spaces between the particles in a close-packed system.

\subsubsection{Color Pigments}

Color pigments are another type of pigment used in coatings. In this study, two types of seven colorants were used-all with different pigments (Table 1). The pigments in the 1900 series colorants were formulated conventionally, with organic solvents. The 8800 series colorants are formulated with zero VOC's. The magenta pigment used in this study is PR 122, a substituted quinacridone pigment. Among the quinacridone pigments, there are a variety of different shades of red available. The specific shade depends on the different substituents on the molecules and the specific crystal structure in which the molecules are arranged (Patton, 1973). As a result of the two methyl groups on the ring structure of PR 122, the pigment has a magenta color (Patton, 1973). Quinacridone pigments (Figure 1) are expensive, but they can be used to provide good exterior durability. In addition, they are nonbleeding and heat and chemical resistant. These properties are attributed to the high degree of intermolecular hydrogen bonding possible between the carbonyl and amino groups in the lattice structure. 
Table 1. List of Colorants and Corresponding CI Pigments used in this Study

\begin{tabular}{|l|l|}
\hline Colorant (Low VOC/Zero VOC) & CI Pigment Name (Pigment) \\
\hline 1921/8821N Phthalo Green & PG7 (Phthalocyanine Green) \\
\hline 1982/8882N Magenta & PR122 (Quinacridone) \\
\hline 1977/8878N Yellow Oxide & PY42 (Yellow Iron Oxide) \\
\hline 1935/8835N Red Oxide & PR101 (Red Iron Oxide) \\
\hline 1913/8813N Yellow & PY74 (Monoarylide Yellow) \\
\hline 1991/8891N Lamp Black & PBK7 (Carbon Black) \\
\hline 1932/8832N Phthalo Blue & PB15:2 (Phthalocyanine Blue) \\
\hline
\end{tabular}

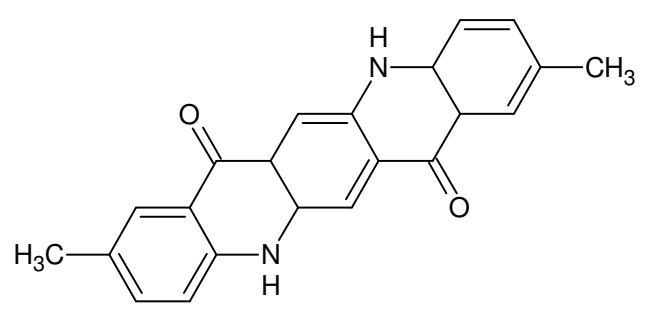

Figure 1. Structure of Pigment Red 122

Both of the green and blue pigments used in this study, phthalocyanine green (PG 7) and phthalocyanine blue (PB 15:2) are based on a copper phthalocyanine structure. The color of phthalocyanine green is imparted by chlorinating the hydrogens on the benzene rings of the phthalocyanine blue structure. The actual structure is a mixture of isomers in which many or all of the hydrogens have been replaced by either chlorine or bromine. By doing this, an improvement in the hue of the applied coating vs. the hydrogen analogue may be realized (Zollinger, 1991). There are three different crystal structures of phthalocyanine pigments used in coatings: alpha, beta, and epsilon. Phthalocyanine pigments have excellent resistance towards acids, bases, and solvents. The alpha structure of phthalocyanine blue will convert to the beta form at temperatures above $200^{\circ} \mathrm{F}$, and this will result in a loss of tint strength and shade of the color. On the other hand, phthalocyanine greens are stable toward heat. The stability of the pigment can be improved further by incorporating basic groups (such as a tertiary amino group) 
as substituents on the benzene rings. These molecules may act as adsorbers of binding agents on the surface of the pigment crystals, providing a sterically stabilized system. This is one of the techniques used to overcome the inherent flocculation problem with phthalocyanine pigments (due to their low surface energy) (Zollinger, 1991). Flocculation and steric stabilization are two topics that will be covered later.

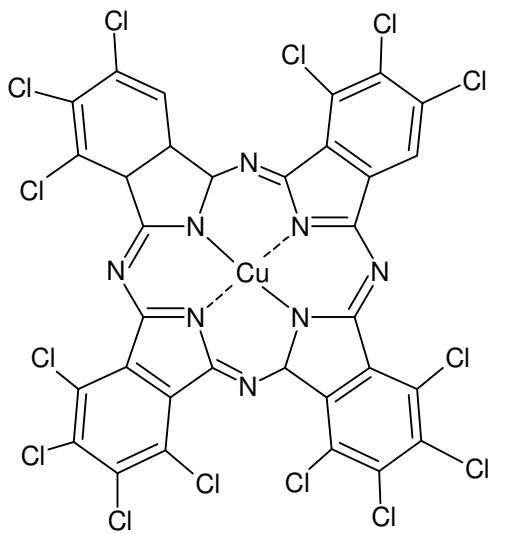

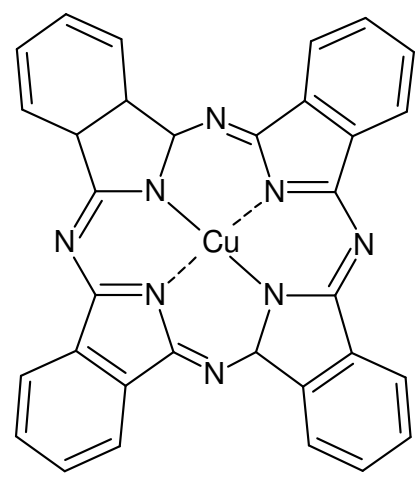

Figure 2. Possible Structure of Pigment Green 7 (left) and Actual Structure of Pigment Blue 15:2 (right)

The yellow and red pigments (PY 42 and PR 101 respectively) used in this study are both derived from synthetic iron oxide. There are four types of synthetic red iron oxide pigments commonly used; all are manufactured differently with different particle sizes and shapes. They all contain the same chemical composition: $\mathrm{Fe}_{2} \mathrm{O}_{3}$. Red iron oxides are exhibit excellent heat resistance; they are also stable toward chemical action (acids/bases) and UV light. Because they reflect almost no light below incident radiation wavelengths of $400 \mathrm{~nm}$, they are useful in protecting binders in various coating compositions against UV degradation. They also provide excellent hiding due to their high refractive indices (2.94-3.22 on average). Yellow iron oxides are composed of hydrated iron oxide particles $\left(\mathrm{Fe}_{2} \mathrm{O}_{3} \cdot \mathrm{xH}_{2} \mathrm{O}\right)$. They have the same excellent bleed resistance, acid/base chemical resistance, and light-fastness properties as their red counterparts, 
but limited heat resistance. When heated above $350^{\circ} \mathrm{F}$, yellow iron oxides are subject to color changes due to the evaporation of the water content.

In addition to the inorganic yellow (PY 42), an organic monoarylide yellow pigment was used (PY 74). These pigments are characterized by the presence of a nitrogen-nitrogen double bond called an azo group $(\mathrm{N}=\mathrm{N})$. Molecules of this type are susceptible to photoisomerization about the $\mathrm{N}=\mathrm{N}$ axis, which can change the color properties (and also explains the weakening of the properties on exposure to sunlight). There are at least 20 different monoarylide yellow pigments known, each with different chemical structures giving different shades of color from greenish-yellow to a deeper reddish-orange. Monoarylide yellows have high chromas, but have poor bleed resistance and may sublime upon exposure to high temperatures. Also, monoarylide yellows have acid-base properties; they may be protonated at the azo group or a nearby amino group (Figure 3) (Zollinger, 1991). The conjugate acid and/or base will have a different color than the parent molecule. They do have some advantages over their inorganic counterparts however; monoarylide yellows have much greater tinting strengths, brighter hues and higher chromas. Their use in aqueous systems is widespread due to their excellent alkaline chemical resistance.<smiles>COc1cc([N+](=O)[O-])ccc1/N=N/C(C(=O)O)C(=O)Nc1ccccc1OC</smiles>

Figure 3. Structure of Pigment Yellow 74

The most non-polar pigment used in this study is PBK 7 Lamp Black. Lamp black pigments are the largest particle size black pigments, with sizes on the order of $0.5 \mu \mathrm{m}$. Because of these large particle sizes, lamp black pigments often result in coatings with a grayish black shade. The carbon black pigments are composed of elemental carbon, arranged in the most stable 6-membered ring conformation. However, their surfaces and specific chemical structure differ 
from pigment to pigment. The surfaces of carbon black pigment particles are often quite polar and sometimes even acidic (Jones, 2007). These properties are a result of the specific method used to manufacture the pigment. Usually, carbon black pigments are oxidized after synthesis. This treatment can result in carbon blacks with up to $15 \%$ oxygen content, resulting in a strongly hydrophilic pigment. Oxidation also increases the porosity of carbon blacks, and so they have relatively large ratios of surface area to volume compared to other pigments. This can result in coatings with high viscosities at low pigment concentrations.

\subsection{Pigment Dispersion}

Before the addition of the pigment to the base (in the form of the color concentrate), the pigment must be dispersed in the color concentrate. Effective dispersion of the pigment in the color concentrate and the coating is one of the most important steps of the coating formulation process. Many aspects of colloid and surface science come into play and must be considered when adding pigment dispersions to coating formulations. Pigments are small, solid particles that must be dispersed in an aqueous medium. Small particles tend to aggregate over time due to the attractive force that comes into play when the molecules collide with each other as a result of Brownian motion. Groups of particles that are held together by an attractive force are called aggregates. The frequency of collisions between particles in a colloidal dispersion is determined by the concentration and the physical properties of the particles (such as the size, surface energy, and shape), the viscosity, and the temperature of the continuous medium. Mysels (Mysels, 1959) used Einstein's law to determine the effect of particle dimensions on the rates of Brownian motion. From his research, he found that smaller particles travel a further distance than larger ones when compared on the same time scale. He also found that larger particles have a higher chance of colliding with one another. These results are important concepts that must be taken into account when formulating pigment dispersions. 
The forces governing attraction between different particles in a coating are almost always van der Waal's interactions. The strength of interaction between two spherical molecules from any van der Waal's force decays exponentially as the particles move further apart (for this discussion, we can safely assume the colloidal particles in a coating are spherical in shape). It is evident that these attractive forces can be strong when the particles are close together. In addition, there also exists a repulsive force between two particles as they approach one another closely. In order for a colloidal system to be stable, the repulsive forces must be stronger than the van der Waal's attractive forces between the particles in a system. DLVO theory (formulated by Derjaguin, Landau, Verwey, and Overbeek) suggests that the stability of a colloidal system is a function of the potential energy of the particles that make up the system (Malvern, 2012). This theory and the attractive force that exists between two particles will be described in more detail in section 1.4.1. As two particles move further apart, there is a secondary minimum in the interaction potential that causes flocculation. Flocculation results in the formation of a loose, open network of particles that may or may not separate from the rest of the system. The difference between flocculation and coagulation is the interparticle distances, which are greater in flocculated systems. Flocculation is a phenomenon that coatings formulators seek to avoid. Because the forces holding particles together in a flocculated system are weaker (as opposed to coagulated particles), flocculation can be reversed by applying low levels of shear. Flocculation not only occurs with pigment particles, but latex particles as well. Although the clusters formed during flocculation can be re-dispersed by applying low levels of shear stress, the properties of a flocculated, dried coating cannot be reversed (Jones, 2007). Flocculation reduces light scattering and hiding as a result due to the larger particle sizes. On the other end of the spectrum, flocculation results in less absorption by the color pigments, which in turn results in lower tinting strengths of the coating. Gloss is also affected in flocculated dispersions. Flocculation leads to higher coating viscosities at low shear rates and therefore shear-thinning properties. Coatings 
formulators have many different tools at their disposal in order to combat flocculation, and they will be discussed below.

There are three steps to formulate a coating with effectively dispersed pigments: wetting, separation, and stabilization. Wetting is the displacement of air, water, and other contaminants from the surface of pigment particles and aggregates. This requires that the surface tension of the vehicle be lower than the surface free energy of the pigment to be dispersed. This is one of the main problems in formulating pigmented waterborne coatings. Because the surface tension of water is so high $(72.7 \mathrm{mN} / \mathrm{m}$ compared to $25-50 \mathrm{mN} / \mathrm{m}$ for typical organic solvents), special additives called surfactants are required in order to lower the surface tension so that wetting may occur. Surfactants are molecules with specific hydrophobic and hydrophilic regions designed to lower the surface tension of a component of a material at the interface with the continuous phase. The next step in pigment dispersion is to separate the particles. Separation is the process by which the pigment aggregates are broken up into individual particles (without grinding the crystals to a smaller particle size). This is accomplished with the use of specific types of machines that apply shear forces to the aggregates suspended in the vehicle. One example of a machine used for pigment separation (which is used in this study) is the high-speed disk disperser. This instrument consists of a rotating disc with blades at the edges, attached to a shaft. The disc is placed in the coating and is made to rotate at high speeds. The shearing force comes from the differential laminar flow rates streaming outwards from the edges of the disk. The final step when making pigment dispersions is to stabilize the particles. Stabilization of the pigment particles is necessary following separation in order to prevent flocculation. In addition to the pigment particles, the latex particles must also be stabilized, because they are susceptible to flocculation as well. Latex particles are supplied as stabilized dispersions. In order to stabilize a pigment or latex particle, the surface of the particles is modified in such a way as to prevent them from closely approaching each other. 
There are two important ways of stabilizing particles: charge and steric stabilization. Charge stabilization can be realized by chemically attaching equivalent ionic groups onto the surface of the particles. When equivalent charges are on the surface of the particles, they will avoid each other due to electrostatic repulsion. Steric stabilization can be conferred by chemically attaching layers of material (usually polymer molecules) on the surface of the particles. When the surface layer polymer particles approach those of another molecule, there is a reduction in the number of possible conformations they may adopt, and hence a localized reduction in entropy. In order to exist in a higher entropic state, the surface layer polymer molecules will not approach those of another molecule closely, and therefore the stabilized particles will not approach each other, remaining evenly dispersed (Jones, 2007). Stabilization requires the addition of a dispersing aid. A dispersing aid is a type of additive whose purpose is solely to keep the pigment and latex particles dispersed. Dispersants adsorb onto the surface of polymer or pigment particles and provide either charge or steric stabilization. Some dispersants are formulated to provide both charge and steric stabilization; this is often referred to as electrosteric stabilization. These materials must adsorb onto the surface of the particles to be stabilized in order to function properly. The process of getting the dispersants to adsorb onto the surface of the latex and/or pigment particles is easier in theory, because in practice, the surfactants and dispersants are in competition for adsorption on the pigment/latex particles with molecules from the continuous phase and other parts of the coating.

Careful attention must be paid to the selection of surfactants in waterborne coatings. Certain surfactants have higher adsorption affinities for certain pigments; at the same time others prefer to remain in the aqueous phase. Surfactants are only soluble up to a certain point in aqueous solution. Above a certain concentration, surfactants will spontaneously assemble in clusters called micelles. This upper solubility limit is called the critical micelle concentration (CMC). In aqueous solution, micelles contain about 30-100 surfactant molecules. The 
hydrophobic ends are oriented toward the center of the micelle, while the hydrophilic ends are pointed outward. The formation of micelles can have a dramatic impact on the dispersion stability of the latex and pigment particles in coatings. Each surfactant has a different proportion of hydrophilic and hydrophobic groups; this is described by an empirical quantity called the hydrophile-lipophile balance (HLB). Every surfactant has a unique HLB value; those with lower values are more hydrophobic and more easily dispersed in non-polar media, while those with higher values are more hydrophilic and are more easily dispersed in water. Hydrophilic pigments such as titanium dioxide are wetted spontaneously in aqueous media and often only need a dispersant to stabilize them (Paranthaman, 2000). Hydrophobic organic pigments often need surfactants of a lower HLB value in order to stabilize them, while inorganic pigments require surfactants of a higher HLB value. In addition to affecting the pigments, different surfactants can impart different rheological characteristics to a coating. The use of anionic surfactants leads to the formation of essentially rigid particles (due to electrostatic stabilization). Latex formulations with anionic surfactants can have low viscosities at high solids contents. Latex formulations with nonionic surfactants have higher viscosities at lower solids contents. The surfaces of nonionic surfactant-stabilized particles are not rigid, and so latex particles stabilized by nonionic surfactants can be distorted when shear stress is applied, imparting shear-thinning characteristics to the coating (Parfitt, 1973).

\subsubsection{DLVO Theory and the Electric Double Layer}

The theory of Derjaguin, Landau, Verwey, and Overbeek (DLVO theory) describes mathematically the interaction force between two colloidal particles in solution. In order to be stable, the steric and electrostatic repulsion forces must be higher than the attractive van der Walls forces to such an extent that an energy barrier of several orders of magnitude higher than the thermal energy of the particles exists (Parfitt, 1973.). All dispersed particles in a continuous medium will acquire a surface charge, and the state of this electric charge is an important factor 
governing the stability of the particles toward flocculation. The charge on the surface of a colloidal particle in a continuous medium will depend on the $\mathrm{pH}$ of the solution. The $\mathrm{pH}$ at which the surface charge is zero is called the isoelectric point (IEP). Below the IEP, the surface has a net positive charge and above the IEP, the surface has a net negative charge. The electrostatic interactions between a surface and the surrounding medium are quite complex, and there are many theories that seek to describe these interactions.

In a colloidal dispersion at equilibrium, ions of opposite charge will migrate toward the surface of the charged colloidal particles, forming what is called an electrical double layer. The electrical double layer is the region in the vicinity of the solid-continuous medium interface in which there is a change in mean potentials or a discontinuity of charge or electrical properties. There are four different mechanisms by which solid particles acquire charge in an aqueous medium; the dissociation of any ionogenic groups present on the particle surface (dispersing agents, surfactants, etc.), the unequal dissolution of oppositely charged ions of which the particle may be composed, if the particle adsorbs ions in solution, or if there is adsorption and orientation of dipolar molecules at the particle interface. These are important concepts to consider when making a stable dispersion of pigment particles in a coating.

There are many theories out there regarding the nature of the electrical double layer. The most widely accepted theory is the Stern model. In the Stern layer, the surface of the solid particle is charged, and is surrounded by a layer of ions of opposite charge at a certain distance away from the surface (usually the radius of the ions). Waterborne coatings usually contain either monomeric or polymeric dispersants, or a combination of both. These dispersants can be charged or nonionic. In the case of charged dispersants, these molecules alter the surface chemistry of the pigment particles and act to extend the distance of the Stern layer out further into solution. The Stern model has been shown to be accurate for a number of colloidal systems. 


\subsection{Thickeners}

Waterborne coatings require a shear-thinning profile because the ideal application viscosity in a shear-thinning fluid corresponds directly with the shear rate of various modes of application for coatings. In the early days, the approach to modify the rheology of the coating to make it shear-thinning was to add water-soluble polymers like hydroxyethylcellulose (HEC). HEC-based polymers thicken the aqueous phase through their contribution to the hydrodynamic volume of the system and by participating in chain entanglements with other components of the system (Glass, 1999). By using HEC polymers of different molecular weights, the rheology of the coating could be tailored for specific applications. These water-soluble HEC polymers were a cheap way to modify the rheology of coatings early on, but their use created problems. Depletion flocculation, an entropically driven process, occurs as a result of the presence of the high molecular weight HEC polymer chains (Glass, 1999). The cellulose polymer chains in the interstitial region between latex particles are restricted with respect to the number of conformations they can adopt (Shay, 1995). By migrating out of the low entropy interstitial region into a less occupied space, they can exist in a higher entropic state. As the cellulose polymer chains migrate away from the latex particles, the latex particles move closer toward each other and flocculation occurs. On a larger scale, this can be seen in coatings formulated with HEC-based thickeners when a clear liquid top layer containing the water-soluble latex forms; this type of separation is called syneresis (Svanholm, 1997). Flocculation also leads to a reduction in gloss and leveling properties of the coating. In addition, flocculation raises the low shear viscosity, giving the coating a more pronounced shear thinning profile. Extensional flow is a mode of flow often encountered in coating application; it occurs when the fluid is deformed as a result of stretching. Extensional flow is increased through the use of coatings with high molecular weight water-soluble polymers like cellulosic thickeners. This extensional flow leads to an increase in roller spatter, which occurs when the coating forms ligaments in the direction of 
extensional shear. Finally, the backbone of HEC-based thickeners is susceptible to enzymatic attack at the linkage sites between adjacent glucose units.

About 30 years ago, companies introduced a different type of thickener designed to overcome the drawbacks from using HEC-based thickeners. These thickeners were called associative thickeners (and subsequently, HEC-based thickeners were referred to as nonassociative thickeners). There are many different types of associative thickeners; they interact with the other parts of the coating differently than non-associative thickeners and also are more complex. All associative thickeners have a water-soluble hydrophilic backbone with two or more hydrophobic groups attached. Associative thickeners thicken the aqueous phase through hydrophobic associations with the surface of latex particles and hydrophobic/hydrophilic associations with other associative thickener polymers. This surface interaction forms a network system, whereby the motion of the latex particles becomes restricted (due to the connecting associative thickeners), causing an increase in the effective volume fraction of the dispersed phase, and therefore an increase in viscosity. They have been shown to produce coatings with ideal rheological properties; however their performance is extremely sensitive to changes in the coating formulation (especially surfactant concentration) (Glass, 1986). The competition for adsorption sites on the latex surface from the surfactants forms the basis for the most widely accepted theory regarding viscosity loss in coatings upon colorant addition. As more and more surfactants adsorb onto the surface of latex and pigment particles, they become more rigid, and so the coating viscosity also increases. However, there exists a critical concentration of surfactant (exceeding the CMC) at which the viscosity of the formulation drops (Mahli, 2003). Fernando (Fernando, R.H., Unpublished Results 1997) measured the viscosity of solutions of Natrosol (non-associative thickener) and Acrysol (associative thickener) with increasing concentrations of Tergitol $15 \mathrm{~S} 7$ surfactant. Increasing the surfactant concentration from $0.1 \%$ to $2.0 \%$ resulted in a decrease in viscosity of the solution. Past the critical surfactant concentration, which was either at 
or below $2.0 \%$ for the last example, the surfactant molecules envelop the associative thickener molecules and screen out any hydrophobic/hydrophilic interactions they once had with the surface of the latex. This results in the breakdown of the network structure imparted by the associative thickeners and the following loss in viscosity. The surfactants will also decrease the strength of the association between the associative thickener molecules themselves, which also contributes to the decrease in viscosity. Each one of these negative aspects correlated with nonassociative thickeners depends on the molecular weight of the polymer, and some can be avoided with the careful selection of two or more thickeners at low and high molecular weights. Depending on the desired application of the coating, associative thickeners or non-associative thickeners may be used.

\subsection{Measuring the Viscosity of Coatings}

The purpose of a polymeric thickener is to increase the viscosity of the coatings. Polymeric thickeners are also known as rheology modifiers. In this study, the viscosity of the coatings was tested with two different instruments; a Brookfield KU (Krebs Units) Viscometer and a Rheometer. Brookfield KU viscometers are instruments with an attached spindle that is inserted into the coating and rotated at 200rpm. The force necessary to turn the paddle is converted by the instrument into Krebs Units (KU), a unit of viscosity unique to paddle viscometers. However, Brookfield KU viscometers only give the viscosity of the coating at one specific rate of deformation. A coating is subject to a wide range of shear forces before, during, and after application. Because of this, the Brookfield KU viscometer only provides a brief glimpse into how the coating will behave during its lifetime. The rheometer can measure the viscosity of a coating over a wide range of shear rates. By measuring the viscosity of the coating over a wide range of shear rates, it is possible to accurately predict how the coating will behave in many applications. To measure the viscosity of the coating, a small sample of the material is 
placed onto a stage and is brought into contact with a rotating disc. The disc rotates, while the instrument measures the torque necessary for each revolution.

There are four main geometries to apply shear in rheological testing: concentric cylinders, cone and plate discs, parallel plate discs, and rectangular torsion geometries. Concentric cylinders are mainly used for low to medium viscosity fluids, and rectangular torsion geometries are used to test soft or rigid solids. The difference between using a parallel plate vs. a cone and plate geometry is the shear rate applied to the material. The shear rate applied to the material in a parallel plate system is not uniform; it increases from the center of the plate as you move toward the outside. This can be a problem for coatings because the viscoelastic properties of coatings (along with other colloidal dispersions) are shear rate dependent. Inaccurate viscosity readings are bound to occur by applying a non-uniform shear rate across the sample. On the other hand, the rate of shear applied to materials with a cone and plate geometry is constant from the middle to the edge of the disc; these geometries are therefore more suited toward the analysis of viscoelastic coatings.

\subsection{Oscillatory Rheology}

The rheometer is a useful tool, and it can be used to study more than just the viscosity of coatings. Oscillatory rheology is a technique in which a sinusoidal deformation is applied to a sample, while the instrument detects the response of the material. The goal of oscillatory rheology is to study the viscoelastic properties of materials. An ordinary elastic solid will respond immediately to an imposed tensile stress in conformance with Hooke's Law, while an ideal viscous material will be permanently deformed as a result of an imposed stress. Viscoelastic materials have properties in between those of a perfectly elastic solid and an ideal liquid. The elastic part of a viscoelastic material will recover from an imposed deformation, however part of the material will be permanently deformed due to the viscous component. Most waterborne 
coatings are viscoelastic materials, and much information can be gained with oscillatory rheology. There are two important parameters that will be examined in this study: the elastic modulus $\left(\mathrm{G}^{\prime}\right)$, which is a measure of the ability of the material to store energy, and the viscous modulus (G'), which is a measure of the ability of the material to dissipate energy. In this study, a dynamic strain deformation at a specific frequency will be imposed on the samples. A typical viscoelastic material will initially respond linearly to an imposed sinusoidal strain. After a certain point (known as the critical strain), G' will start to decrease, while G' will increase. Within the linear viscoelastic region, G' and G" are independent of the applied strain. The imposed strain is not high enough to disrupt the coating suspension. At the critical strain, the coating network is disrupted, resulting in a decrease in G' and a slight increase in G'. After determining the linear viscoelastic region, the second part of the test in this study is to determine the rate-dependant effects of shear deformation on the samples. This is done by running a frequency sweep test. This test involves imposing a deformation on the material at a certain percent strain (within the linear viscoelastic region) at an increasing frequency as the test progresses. This allows the researcher to study the rate-dependant shear deformation effects on a material. Because the test is run within the linear viscoelastic region, the test can be done without disrupting the microstructure of the sample.

\subsection{Objective of Study}

Coatings formulated with associative thickeners are sensitive to formulation variables, especially surfactant concentration. When colorant is added to waterborne coating bases, the surfactants in the colorant interfere with the thickening mechanism of the associative rheology modifiers, which causes the viscosity of the coating to decrease significantly and dramatically alters the coating's rheological properties. Typical waterborne coatings are formulated to have viscosities in the neighborhood of $100 \mathrm{Krebs}$ units before colorant addition. Upon the addition of colorant, the viscosity of the coating typically decreases by 30-35 Krebs units, but there have 
been reports of decreases as high as 38 Krebs units. This is an important effect because the viscosity of the coating as a function of shear rate affects the ease of brushing the coating onto a substrate, the film thickness, the sag and leveling properties, and settling. The purpose of this research is to characterize the effects of colorant addition on the rheological properties of waterborne coatings in an attempt to explain the changes in rheology of coatings upon tinting, and to serve as a basis for future research.

\section{Materials and Methods}

\subsection{Effect of Colorant Addition on Rheology of Pastel Bases with Associative and Non- associative Thickeners}

\subsubsection{Pastel Base Formulation with HEC Thickener}

The formula used in this study was based on the EPS 2792 Interior White Semi-Gloss

Pastel Base, with acceptable substitutions made according to the materials available (Table 2). Instead of EPS 2792, a different acrylic-emulsion latex was used: EPS 2757. EPS 2757 is formulated with no APEO raw materials and is designed for use in high performance zero-VOC paints. Both pastel bases were formulated to be as similar as possible, with the exception of the thickener. Of particular importance is that the formulations were made with a high molecular weight polyacrylic acid dispersant (Tamol 850) and a silicone surfactant (BYK-348). Also, the thickeners used were both in the middle of the molecular weight range of available Natrosol HEC thickeners (Natrosol 250HR being a little higher than Natrosol 250GR). All ingredients in the grind were added in succession and dispersed to a Hegman of 7.5, followed by sequential addition of the letdown ingredients. After the formulation was completed, the Stormer viscosity was measured, and then measured again after 72 hours at rest. Following this, the solids fraction was determined by following the procedure outlined in ASTM D2369. 
Table 2. Pastel Base with HEC Formulation

\begin{tabular}{|c|c|c|}
\hline Raw Material & Chemical Function & Weight in Formulation (g) \\
\hline \multicolumn{3}{|l|}{ Grind } \\
\hline Water & Continuous medium & 50.2 \\
\hline Natrosol 250GR & Non-associative thickener & 50.2 \\
\hline Tamol 850 & Dispersing agent & 4.70 \\
\hline$B Y K-348$ & Surfactant & 2.10 \\
\hline$B Y K-023$ & Surfactant & 4.0 \\
\hline Nuosept 95 & Biocide & 3.30 \\
\hline Minex-10 & Mineral for tint retention, brightness & 10.07 \\
\hline TiPure R902 & White pigment & 229.9 \\
\hline \multicolumn{3}{|l|}{ Let-Down } \\
\hline EPS-2757 & Acrylic emulsion latex & 448.2 \\
\hline Propylene Glycol & Freeze-thaw agent, coalescing aid & 4.56 \\
\hline Texanol & Coalescing aid & 9.35 \\
\hline$B Y K-023$ & Surfactant & 8.1 \\
\hline Ammonium Hydroxide & Used to adjust $\mathrm{pH}$ of formulation & 1.1 \\
\hline Natrosol 250GR & Low MW Non-associative thickener & 125.8 \\
\hline Natrosol 250HR & High MW Non-associative thickener & 143.7 \\
\hline Total Weight & & 1095.3 \\
\hline \multicolumn{3}{|c|}{ Properties } \\
\hline \multicolumn{2}{|c|}{$\%$ Non-Volatiles by Weight (\%NVW) } & 44.09 \\
\hline \multicolumn{2}{|c|}{$\%$ Non-Volatiles by Volume (\%NVV) } & 32.61 \\
\hline \multicolumn{2}{|c|}{ PVC (Pigment Volume Concentration) } & 21.74 \\
\hline
\end{tabular}




\subsubsection{Pastel Base Formulation with HEUR Thickener}

The HEUR-thickened base was formulated with a combination of two nonionic HEUR thickeners: Acrysol RM-2020NPR (for high shear viscosity) and Acrysol SCT-275 (for low-shear viscosity). All ingredients in the grind were added in order and then dispersed to a Hegman of 7.5. This was followed by the sequential addition of the ingredients in the letdown. After the formulation was completed, the same procedures were used as with the HEC-thickened sample to obtain the Stormer viscosity and solids content.

Table 3. Pastel Base with HEUR Formulation

\begin{tabular}{|c|c|c|}
\hline Raw Material & Chemical Function & $\begin{array}{c}\text { Weight in Formulation } \\
(\mathrm{g})\end{array}$ \\
\hline \multicolumn{3}{|l|}{ Grind } \\
\hline Water & Continuous medium & 90.1 \\
\hline Acrysol RM-2020NPR & Associative thickener & 10.0 \\
\hline Tamol 850 & Dispersing agent & 5.4 \\
\hline BYK-348 & Surfactant & 2.029 \\
\hline BYK-023 & Surfactant & 4.5 \\
\hline Nuosept 95 & Biocide & 3.2 \\
\hline Minex-10 & Mineral for tint retention, brightness & 10.05 \\
\hline TiPure R902 & White pigment & 230.3 \\
\hline \multicolumn{3}{|l|}{ Let-Down } \\
\hline$E P S-2757$ & 50.00 & 448.7 \\
\hline Water & 0.00 & 227.5 \\
\hline Propylene Glycol & 0.00 & 4.5 \\
\hline Texanol & 0.00 & 9.04 \\
\hline$B Y K-023$ & 18.50 & 7.50 \\
\hline Ammonium Hydroxide & 0.00 & 1.4 \\
\hline Acrysol RM-2020NPR & 20.00 & 11.5 \\
\hline Acrysol SCT-275 & 17.50 & 8.2 \\
\hline Total Weight & & 1073.9 \\
\hline \multicolumn{3}{|c|}{ Properties } \\
\hline \multicolumn{2}{|c|}{$\%$ Non-Volatiles by Weight (\%NVW) } & 44.64 \\
\hline \multicolumn{2}{|c|}{$\%$ Non-Volatiles by Volume (\%NVV) } & 32.08 \\
\hline \multicolumn{2}{|c|}{ Pigment Volume Concentration (PVC) } & 22.04 \\
\hline
\end{tabular}




\subsubsection{Colorant Addition}

Two sets of seven colorants were used for the study on the EPS 2757 Pastel and Deeptone Bases; one set was from the CCA 1900 line of low-VOC colorants, and in the second set were the corresponding zero-VOC colorants from the NovoColor® II line. All in all, 28 samples were prepared (14 with the HEC-thickened base and 14 with the HEUR thickened base). The chemical compositions of the colorants were not available, but the main pigments used in each of the samples are outlined in table 1 . Approximately $40 \mathrm{~g}$ of the pastel base were weighed into a plastic mixing cup, followed by five grams of colorant. The samples were mixed in a Thinky AR100 speed mixer for 60 seconds, stirred vigorously with a spatula for an additional 20 seconds, and then transferred to a 4oz. glass jar. The viscosity profile of each sample was obtained approximately 24 hours following colorant addition

\subsubsection{Viscosity Measurements and Viscoelastic Characterization}

The viscosity profile of each sample was obtained at $25^{\circ} \mathrm{C}$ with a TA Instruments AR2000 rheometer equipped with a 40mm, $1^{\circ}$ cone. All experiments were run with the tip of the cone kept at a distance of $55 \mu \mathrm{m}$ from the Peltier plate. The rheometer was run in a steady-state flow mode from $0.02-200 \mathrm{~s}^{-1}$ shear rate. Each sample was stirred gently for about 15 seconds with a spatula before analysis. The samples were designated by an $\mathrm{N}$ (for Natrosol), followed by the colorant designation for the HEC-thickened samples. The same naming procedure was used to designate the HEUR-thickened samples, which were represented by an A (for Acrysol) (for example, N-1921 would be the sample formulated with HEC and 1921 green colorant and A$8882 \mathrm{~N}$ would be the sample formulated with HEUR and 8882N magenta colorant).

Viscoelastic properties were determined by oscillatory measurements as a function of the shear strain of each sample. Each sample was run in the direction of increasing percent shear strain, from $0.01-100 \%$ at $1 \mathrm{~Hz}$ frequency. Immediately following this, frequency sweeps were performed at percent strains located within the linear viscoelastic region (LVR) of each sample. Each frequency sweep test was run in the increasing direction, from $0.01 \mathrm{~Hz}$ to $100 \mathrm{~Hz}$ at the 
chosen strain percentage value. All HEC-thickened samples were run at $0.1 \%$ strain, while the chosen value for the HEUR-thickened samples was varied and is indicated in the graphs.

\subsubsection{Gloss and Tint Strength Measurements}

Immediately following the rheology analysis, drawdowns of each sample were obtained alongside their parent bases at a thickness of 6 millimeters. At least 24 hours of drying time were allowed for all drawdowns. Tristimulus values were measured with a Datacolor Mercury spectrophotometer using a D65 standard illuminant. Tint strengths were calculated according to the procedure outlined in ASTM D4838-88 using both pastel base drawdowns as standards for each set of samples. The contrast ratios were also determined from these samples. Contrast ratios were measured as the reflectance of the coating over the black portion of the Leneta chart divided by their reflectance over the white portion. Gloss values were recorded as the average of three measurements at $60^{\circ}$ and $85^{\circ}$ incident angles with a BYK-Gardner micro-Tri-glossmeter.

\subsection{Effect of Colorant Addition on Rheology of Deeptone Bases with Associative and}

\section{Non-associative Thickeners}

\subsubsection{Deeptone Base Formulation with HEC Thickener}

Both formulations used in this study were based on the pastel bases made above. In order to make them deeptone bases, the $\mathrm{TiO}_{2}$ in each formulation was replaced with latex (Table 4). The deeptone bases were also formulated to be as similar as possible, with the exception of the thickener. All ingredients in the grind were added in succession and dispersed with a high-speed disk disperser. This was followed by the sequential addition of the letdown ingredients. After the formulation was completed, the Stormer viscosity was measured, and then measured again after 72 hours at rest. The solids fraction in each base was determined by following the procedure outlined in ASTM D2369. 
Table 4. Deeptone Base with HEC Formulation

\begin{tabular}{|c|c|c|}
\hline Raw Material & Chemical Function & Weight In Formulation (g) \\
\hline \multicolumn{3}{|l|}{ Grind } \\
\hline Water & Continuous medium & 61.30 \\
\hline Natrosol 250GR & Non-associative thickener & 68.10 \\
\hline Tamol 850 & Dispersing agent & 0.70 \\
\hline$B Y K-348$ & Surfactant & 2.91 \\
\hline$B Y K-023$ & Surfactant & 8.30 \\
\hline Nuosept 95 & Biocide & 4.10 \\
\hline Minex-10 & Mineral for tint retention, brightness & 13.60 \\
\hline \multicolumn{3}{|l|}{ Let-Down } \\
\hline$E P S-2757$ & Acrylic emulsion latex & 611.00 \\
\hline Propylene Glycol & Freeze-thaw agent, coalescing aid & 5.93 \\
\hline Texanol & Coalescing aid & 15.70 \\
\hline$B Y K-023$ & Surfactant & 8.10 \\
\hline $\begin{array}{c}\text { Ammonium } \\
\text { Hydroxide }\end{array}$ & Used to adjust $\mathrm{pH}$ of formulation & 1.50 \\
\hline Natrosol 250GR & Low MW Non-associative thickener & 102.7 \\
\hline Natrosol $250 H R$ & High MW Non-associative thickener & 139.8 \\
\hline Total Weight & & 1049.7 \\
\hline \multicolumn{3}{|c|}{ Properties } \\
\hline \multicolumn{2}{|c|}{$\%$ Non-Volatiles by Weight (\%NVW) } & 32.47 \\
\hline \multicolumn{2}{|c|}{$\%$ Non-Volatiles by Volume (\%NVV) } & 30.80 \\
\hline \multicolumn{2}{|c|}{ Pigment Volume Concentration (PVC) } & 1.73 \\
\hline
\end{tabular}

2.2.2 Deeptone Base Formulation with HEUR Thickener

The HEUR-thickened base was formulated with a combination of two nonionic HEUR thickeners: Acrysol RM-2020NPR (for high shear viscosity) and Acrysol SCT-275 (for low-shear viscosity) (Table 5). All ingredients in the grind were added in order and then dispersed with a high-speed disk disperser. This was followed by the sequential addition of the ingredients in the 
letdown. After the formulation was completed, the same procedures were used as with the HECthickened sample to obtain the Stormer viscosity and solids content.

Table 5. Deeptone Base with HEUR Formulation

\begin{tabular}{|c|c|c|}
\hline Raw Material & Chemical Function & Weight In Formulation (g) \\
\hline \multicolumn{3}{|l|}{ Grind } \\
\hline Water & Continuous medium & 126.1 \\
\hline Natrosol 250GR & Non-associative thickener & 14.1 \\
\hline Tamol 850 & Dispersing agent & 0.70 \\
\hline BYK-348 & Surfactant & 2.94 \\
\hline BYK-023 & Surfactant & 9.3 \\
\hline Nuosept 95 & Biocide & 4.3 \\
\hline Minex-10 & Mineral for tint retention, brightness & 14.1 \\
\hline \multicolumn{3}{|l|}{ Let-Down } \\
\hline EPS-2757 & Acrylic emulsion latex & 628.30 \\
\hline Water & Continuous medium & 179.60 \\
\hline Propylene Glycol & Freeze-thaw agent, coalescing aid & 6.11 \\
\hline Texanol & Coalescing aid & 12.70 \\
\hline BYK-023 & Surfactant & 7.50 \\
\hline Ammonium Hydroxide & Used to adjust $\mathrm{pH}$ of formulation & 1.50 \\
\hline Acrysol RM-2020NPR & Associative thickener & 14.10 \\
\hline Acrysol SCT-275 & High MW Non-associative thickener & 17.90 \\
\hline Total Weight & & 1039.25 \\
\hline \multicolumn{3}{|c|}{ Properties } \\
\hline \multicolumn{2}{|c|}{$\%$ Non-Volatiles by Weight (\%NVW) } & 33.43 \\
\hline \multicolumn{2}{|c|}{$\%$ Non-Volatiles by Volume (\%NVV) } & 31.03 \\
\hline \multicolumn{2}{|c|}{ Pigment Volume Concentration (PVC) } & 1.75 \\
\hline
\end{tabular}




\subsubsection{Colorant Addition}

The CCA colorants used in the pastel base study were also used here. Again, 28 samples were prepared (14 with the HEC-thickened base and 14 with the HEUR thickened base). The pigments used in each of the colorants are outlined in table 1 . Approximately $40 \mathrm{~g}$ of the pastel base were weighed into a plastic mixing cup, followed by five grams of colorant. The samples were mixed in a Thinky AR100 speed mixer for 60 seconds, stirred vigorously with a spatula for an additional 20 seconds, and then transferred to a 4oz. glass jar. The viscosity profile of each sample was obtained approximately 24 hours following colorant addition.

\subsubsection{Viscosity Measurements and Viscoelastic Characterization}

The viscosity profile of each sample was obtained at $25^{\circ} \mathrm{C}$ with a TA Instruments AR2000 rheometer equipped with a 40mm, $1^{\circ}$ cone. All experiments were run with the tip of the cone kept at a distance of $55 \mu \mathrm{m}$ from the Peltier plate. The rheometer was run in a steady-state flow mode from $0.02-200 \mathrm{~s}^{-1}$ shear rate. Each sample was stirred gently for about 15 seconds with a spatula before analysis. The samples were designated by an $\mathrm{N}$ (for Natrosol), followed by the colorant designation for the HEC-thickened samples. The same naming procedure was used to designate the HEUR-thickened samples, which were represented by an A (for Acrysol).

Viscoelastic properties were determined by oscillatory measurements as a function of the shear strain of each sample. Each sample was run in the direction of increasing percent shear strain, from $0.01-100 \%$ at $1 \mathrm{~Hz}$ frequency. Immediately following this, frequency sweeps were performed at percent strains located within the linear viscoelastic region (LVR) of each sample. Each frequency sweep test was run in the increasing direction, from $0.01 \mathrm{~Hz}$ to $100 \mathrm{~Hz}$ at the chosen strain percentage value. In this study, $0.1 \%$ strain was a common value within the LVR of each sample, and so frequency sweeps on all deeptone samples were run at $0.1 \%$ strain.

\subsubsection{Gloss and Tint Strength Measurements}

Immediately following the rheology analysis, drawdowns of each sample were obtained alongside their parent bases at a thickness of 6 millimeters. At least 24 hours of drying time were 
allowed for all drawdowns. Tristimulus values were measured with a Datacolor Mercury spectrophotometer using a D65 standard illuminant. Tint strengths were calculated according to the procedure outlined in ASTM D4838-88 using both deeptone base drawdowns as standards for each set of samples. The contrast ratios were also determined from these samples. Contrast ratios were measured as the reflectance of the coating over the black portion of the Leneta chart divided by their reflectance over the white portion. Because of the lack of hiding power resulting from the absence of $\mathrm{TiO}_{2}$, the only samples to achieve a contrast ratio sufficient to measure the tint strength (contrast ratios of 0.98 are required) were those with red, yellow oxide, and black colorants. In order to increase the hiding power, coating drawdowns were made for the rest of the samples at 15 millimeters thickness. The contrast ratio of each of these samples was determined after drying, but none were found to be sufficient to measure the tint strength. A final attempt was made to produce drawdowns with enough hiding power to measure the tint strength by making drawdowns with the samples N-1921 green and A-1913 yellow at 21 millimeters thickness, but these were also found to be insufficient. Gloss values were recorded as the average of three measurements at $60^{\circ}$ and $85^{\circ}$ incident angles on the 6 millimeter samples with a BYK-Gardner micro-Tri-glossmeter.

\subsection{Effects of Colorant Addition on Rheology of Paints with Different Coalescing Aids}

\subsubsection{DOW Base Formulation}

All materials used to formulate the coating bases were purchased by industrial suppliers. The DOW paint bases were formulated with an acrylic emulsion latex. Sodium dioctyl sulfosuccinate, an anionic compound, was used as the surfactant in the DOW formulation. In addition, an anionic and a nonionic HEUR type rheology modifier were used. All ingredients were added in the order of the formulation list (minus the coalescing aid; see Table 6) under shear in order to make a $2500 \mathrm{~g}$ master batch. This master batch was divided into six separate batches of 
$350 \mathrm{~g}$ each $(700 \mathrm{~g}$ was allotted for the batch with Texanol coalescing aid). Five different coalescing aids (Fig. 4) were added to the auxillary batches, while the sixth served as a control. The coalescing aids used were dioctyl succinate (DOSx), trimethylpentanediol monoisobutyrate (Texanol), 2, 2, 4-trimethyl-1, 3-pentanediol diisobutyrate (TXIB), Optifilm Enhancer 400 (OE400), and dipropylene glycol n-butyl ether (DPnB). The Stormer viscosity of each sample was obtained once immediately after dispersing the coalescing aid, and again after allowing the sample to rest for 24 hours.

Table 6. DOW SG-30-1 Formulation

\begin{tabular}{|c|c|c|}
\hline Raw Material & Chemical Function & Weight in Formulation (g) \\
\hline \multicolumn{3}{|l|}{ Grind } \\
\hline $\mathrm{TiO}_{2}$ slurry & Dispersed white pigment & 775.4 \\
\hline Water & Continuous medium & 188.9 \\
\hline Propylene Glycol & Freeze-thaw agent, coalescing aid & 82.42 \\
\hline Kathon LX $1.5 \%$ & Biocide & 4.288 \\
\hline \multicolumn{3}{|l|}{ Letdown } \\
\hline Rhoplex SG-30 & Acrylic emulsion latex & 1194.0 \\
\hline Coalescing Aid & Coalescing aid & 29.78 \\
\hline Aerosol OT-75 & Surfactant & 3.573 \\
\hline BYK-022 & Defoamer & 5.002 \\
\hline Acrysol RM-2020NPR & Associative thickener & 41.21 \\
\hline Acrysol SCT-275 & Associative thickener & 38.59 \\
\hline Water & Continuous medium & 134.1 \\
\hline Total Weight & & 2500 \\
\hline \multicolumn{3}{|c|}{ Properties } \\
\hline \multicolumn{2}{|l|}{ Total VOC $(\mathrm{g} / \mathrm{l})$} & 150 \\
\hline \multicolumn{2}{|l|}{ Density (lb/gal) } & 10.49 \\
\hline \multicolumn{2}{|c|}{$\%$ Non-Volatiles by Volume (\%NVV) } & 33.97 \\
\hline \multicolumn{2}{|c|}{ Pigment Volume Concentration (PVC) } & 21.95 \\
\hline
\end{tabular}




\subsubsection{Turk Base Formulation}

The Turk paint bases were formulated with an acrylic polymer latex. A hydrophobic surfactant $(\mathrm{HLB}=1.6)$ containing no alkylphenyl ethoxylates was used in addition to two polyurethane associative thickeners. The ingredients for the grind were added in the order of the formulation list under shear. Following addition of the Attagel 50 rheology modifier, the mixture was sheared for 30 minutes to a Hegman Grind of 7 . The ingredients of the letdown were also added in order of the formulation list under shear. As with the Dow paint base, $2500 \mathrm{~g}$ total of the Turk formulation was made and divided into 6 different batches of $350 \mathrm{~g}$ each $(700 \mathrm{~g}$ for the sample with Texanol coalescing aid). The same five coalescing aids (Figure 4) were added to the Turk sample batches under shear, while the sixth served as a control. The Stormer viscosity of each sample was obtained once immediately after dispersing the coalescing aid, and again after the sample was at rest for 24 hours.

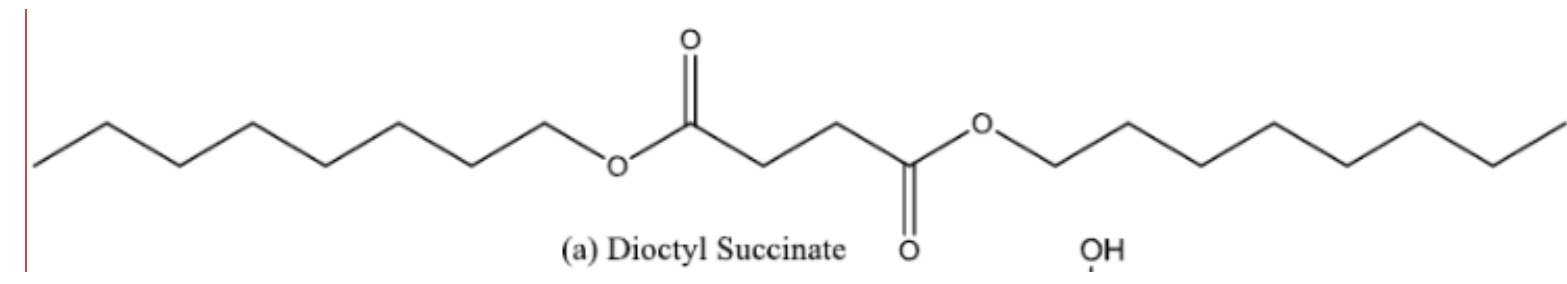

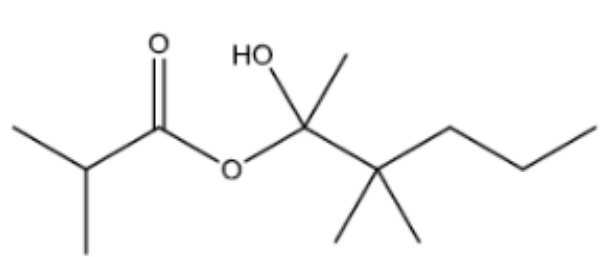

(b) Texanol

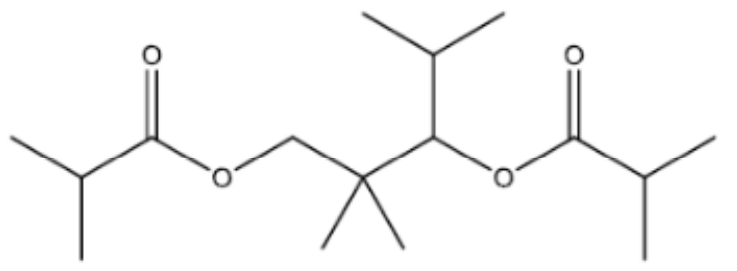

(c) TXIB

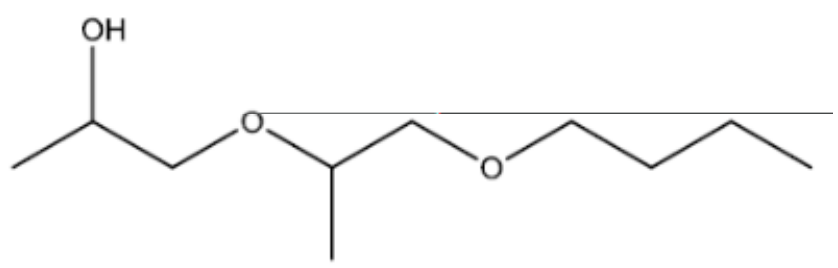

(d) $\mathrm{DPnB}$

Figure 4. Structures of coalescing aids used in this study: (a) DOSx, (b) TX, (c) TXIB, (d) DPnB. (Note: no structure was available for Optifilm Enhancer 400) 
Table 7. Turk Semi-Gloss Formulation

\begin{tabular}{|c|c|c|}
\hline Raw Material & Chemical Function & Weight In Formulation (g) \\
\hline \multicolumn{3}{|l|}{ Grind } \\
\hline Water & Continuous medium & 68.79 \\
\hline Tamol 731A & Scale inhibitor & 18.98 \\
\hline DeIonic LF 80MOD & Surfactant-wetting agent & 4.744 \\
\hline Dee Fo 3010A & Defoamer & 1.186 \\
\hline Tafigel PUR 80 & Associative thickener & 40.32 \\
\hline Minex 10 & Mineral for tint retention, brightness & 118.6 \\
\hline Attagel 50 & Non-associative thickener & 7.116 \\
\hline Water & Continuous medium & 192.1 \\
\hline \multicolumn{3}{|l|}{ Letdown } \\
\hline $\mathrm{TiO}_{2}$ slurry & Dispersed white pigment & 664.1 \\
\hline Water & Continuous medium & 189.8 \\
\hline Tafigel PUR 80 & Associative thickener & 28.46 \\
\hline Ropaque Ultra & $\begin{array}{l}\text { Film-forming, light-scattering } \\
\text { polymer }\end{array}$ & 142.3 \\
\hline Orgal P850RR & Acrylic emulsion latex & 972.5 \\
\hline Coalescing Aid & Coalescing aid & 23.72 \\
\hline Dee Fo 3010A & Defoamer & 3.558 \\
\hline Tafigel PUR 61 & Associative thickener & 23.72 \\
\hline Total Weight & & 2500 \\
\hline \multicolumn{3}{|c|}{ Properties } \\
\hline \multicolumn{2}{|l|}{ Total VOC $(\mathrm{g} / \mathrm{l})$} & 4 \\
\hline \multicolumn{2}{|c|}{ Density (lb/gal) } & 10.54 \\
\hline \multicolumn{2}{|c|}{$\%$ Non-volatiles by Weight (\%NVW) } & 48.79 \\
\hline \multicolumn{2}{|c|}{$\%$ Non-volatiles by Volume (\%NVV) } & 36.84 \\
\hline \multicolumn{2}{|c|}{ Pigment Volume Concentration (PVC) } & 34.09 \\
\hline
\end{tabular}




\subsubsection{Colorant Addition}

All colorants used in this study were provided by Color Corporation of America. The blue, yellow, and red colorants used phthalo blue (PB15:2), medium yellow (blend), and red oxide (PR101) pigments respectively. Detailed descriptions of the ingredients in each colorant formulation were not available. From each batch of the DOW and Turk formulations above, $3 \times 40 \mathrm{~g}$ aliquots were sampled. Each of these samples was mixed with $5 \mathrm{~g}$ of colorant (one red, one yellow, one blue, for each coalescing aid sample) for 30 seconds on a Thinky AR100 conditioning mixer, followed by 30 seconds of mild stirring with a spatula. The 48 total samples were allowed at least 24 hours to equilibrate before rheological analysis.

\subsubsection{Viscosity Measurements}

The viscosity profile of each sample was obtained at $25^{\circ} \mathrm{C}$ with a TA Instruments AR2000 rheometer equipped with a 40mm, $1^{\circ}$ cone. All experiments were run with the tip of the cone kept at a distance of $55 \mu \mathrm{m}$ from the Peltier plate. The rheometer was run in a steady-state flow mode from $0.02 \mathrm{~s}^{-1}-200 \mathrm{~s}^{-1}$. Each sample was stirred gently for about 15 seconds with a spatula before analysis.

\section{Results and Discussion}

\subsection{Effect of Colorant Addition on Rheology of Pastel Bases}

\subsubsection{Viscosity Profiles of Nontinted Bases}

The solids content of HEUR-thickened and HEC-thickened pastel base formulations were similar to what was calculated based upon the weight of the ingredients added (Table 1,2). Since solids content has a large impact on rheology and gloss, it was important to formulate the bases to be as similar as possible. The HEUR-thickened base had a much higher Stormer viscosity than the HEC-thickened base (Table 7). This is because the strength of the hydrophobic network 
imparted by associative thickeners is stronger than a network built upon high MW polymer chain entanglement (given similar formulations). The viscosity profiles of each base show that with the exception in the extreme low shear viscosity region, the viscosity imparted by the HEUR thickeners remains higher than the samples with HEC thickeners. The shear rates corresponding to the Stormer viscosities (approximately $10-30 \mathrm{~s}^{-1}$ ) of each base are indicated.

Table 8. Stormer viscosity and solids content of Pastel Bases

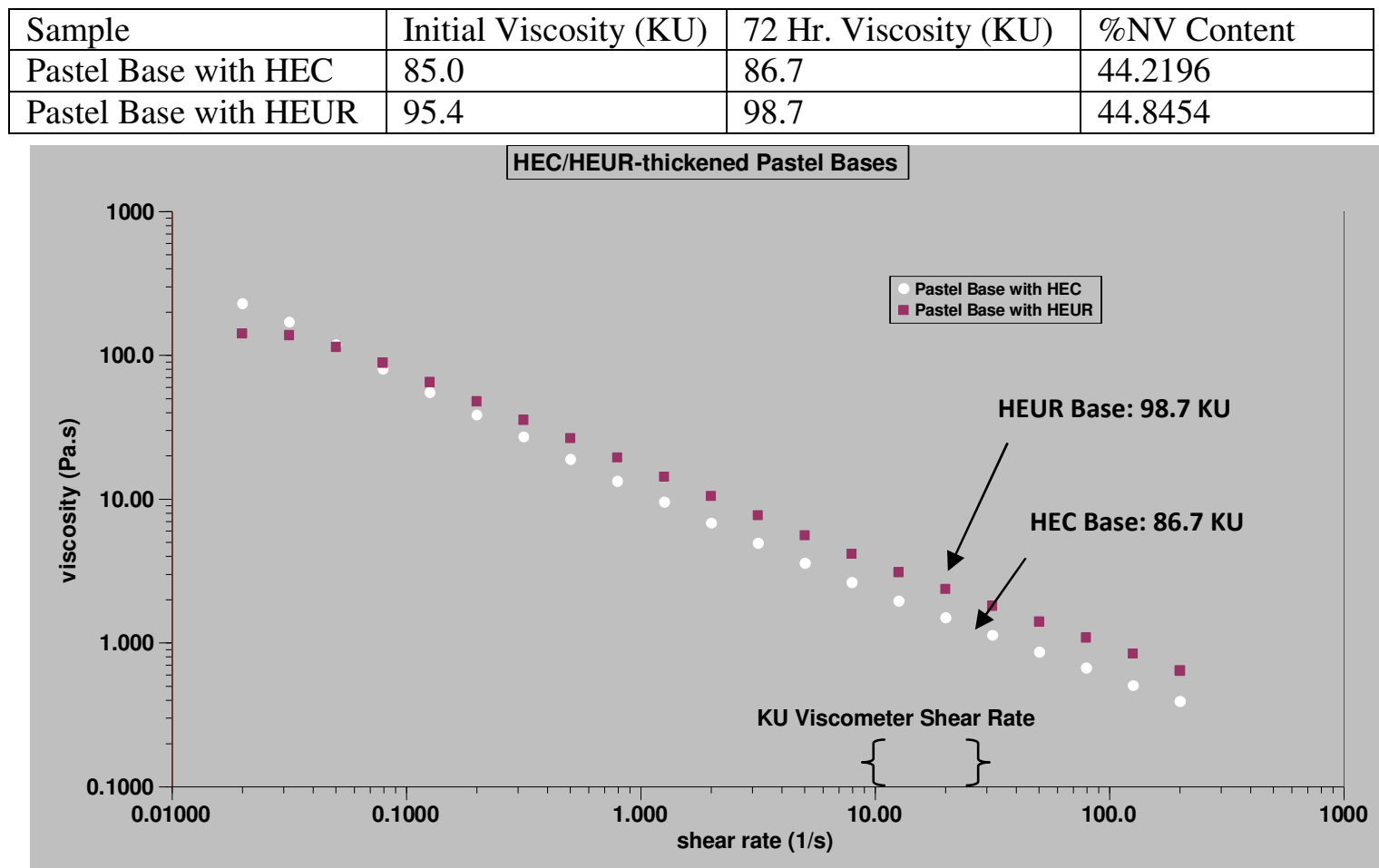

Figure 5. Viscosity profiles of pastel bases with associative and non-associative thickeners

3.1.2 Viscosity Profiles of Samples Arranged by Thickener and Colorant Series

With the exception of the HEC-thickened samples mentioned above, it can be seen (Appendix 1) that HEC-thickened coatings are not influenced by surfactant level. The viscosity profiles of the HEUR-thickened samples were, for the most part, randomly distributed. Generally speaking, the red and black samples were lower in viscosity while both yellows (organic and 
inorganic) were higher in viscosity. One possible explanation for this is there is a difference in the crystal structure and particle size of the pigments in these samples.

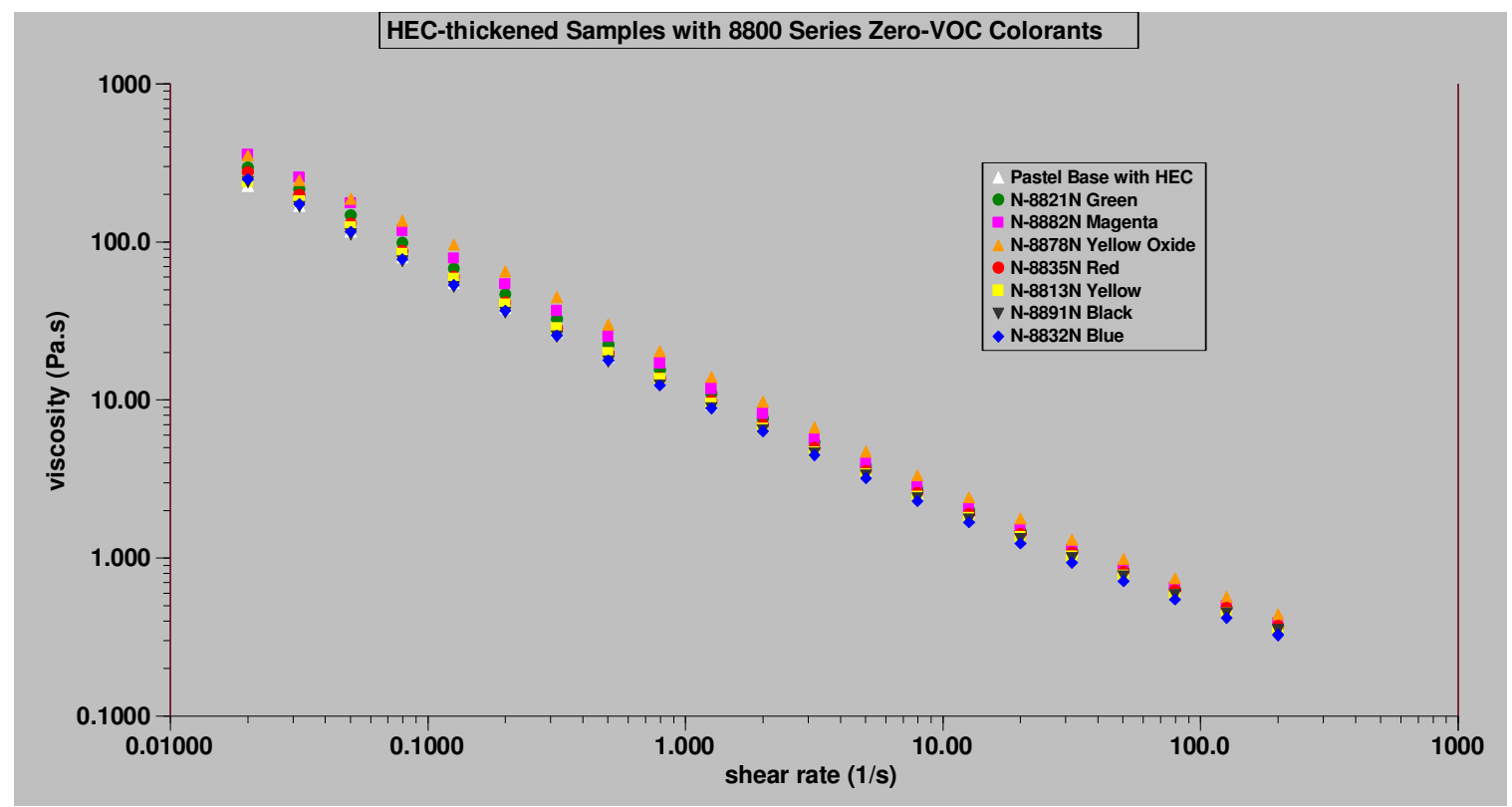

(a)

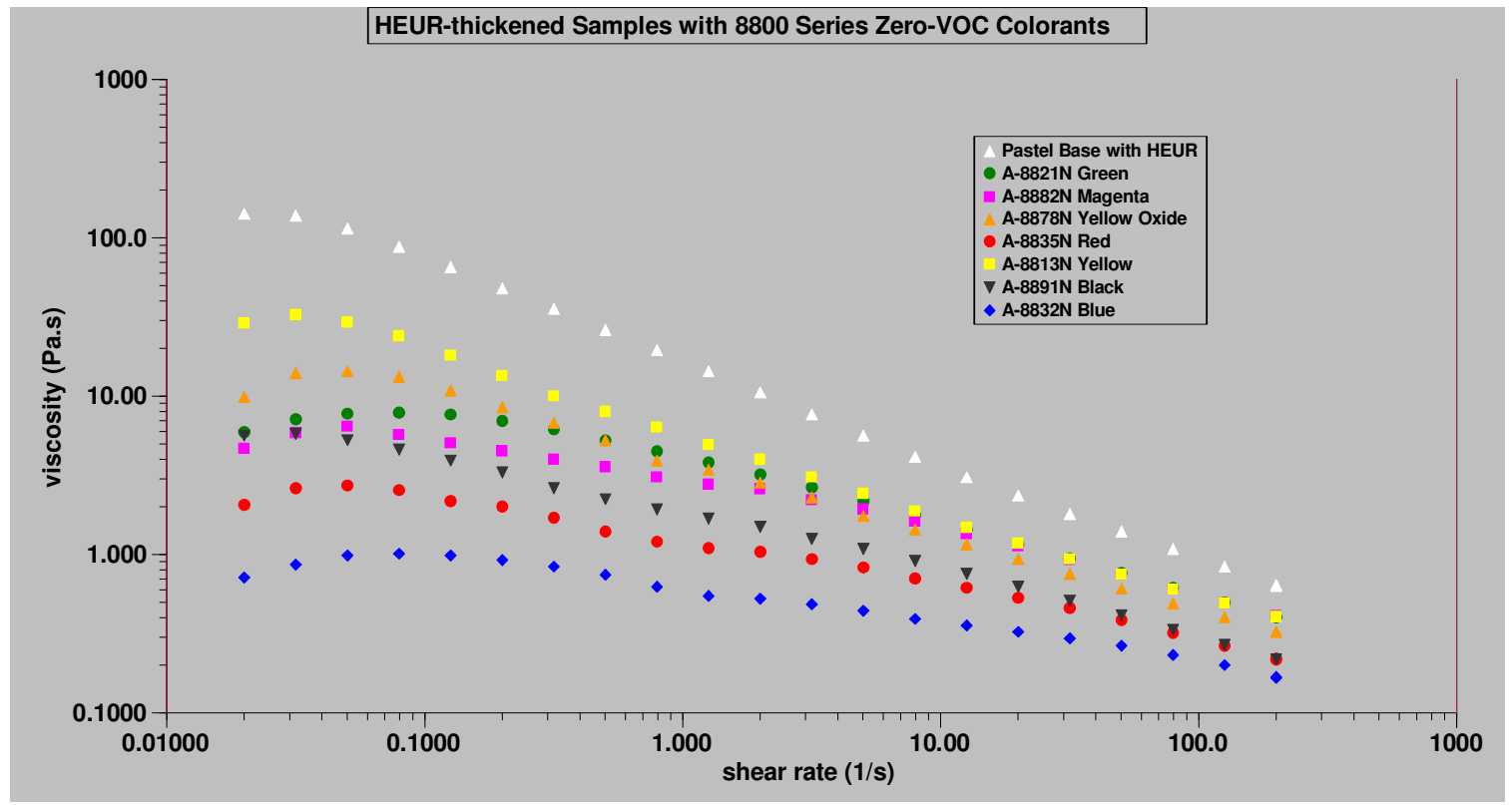

(b)

Figure 6. Viscosity profiles of samples by thickener and colorant series: (a) HEC-thickened samples and (b) HEUR-thickened samples (both with 8800 series zero-VOC colorant) 
3.1.3 Viscosity profiles of samples arranged by color

The phenomenon of viscosity loss upon tinting was evident in only the samples

formulated with the associative thickeners. Most of the samples formulated with the cellulosic thickeners were overlapping each other (Appendix 2, Figure 7).

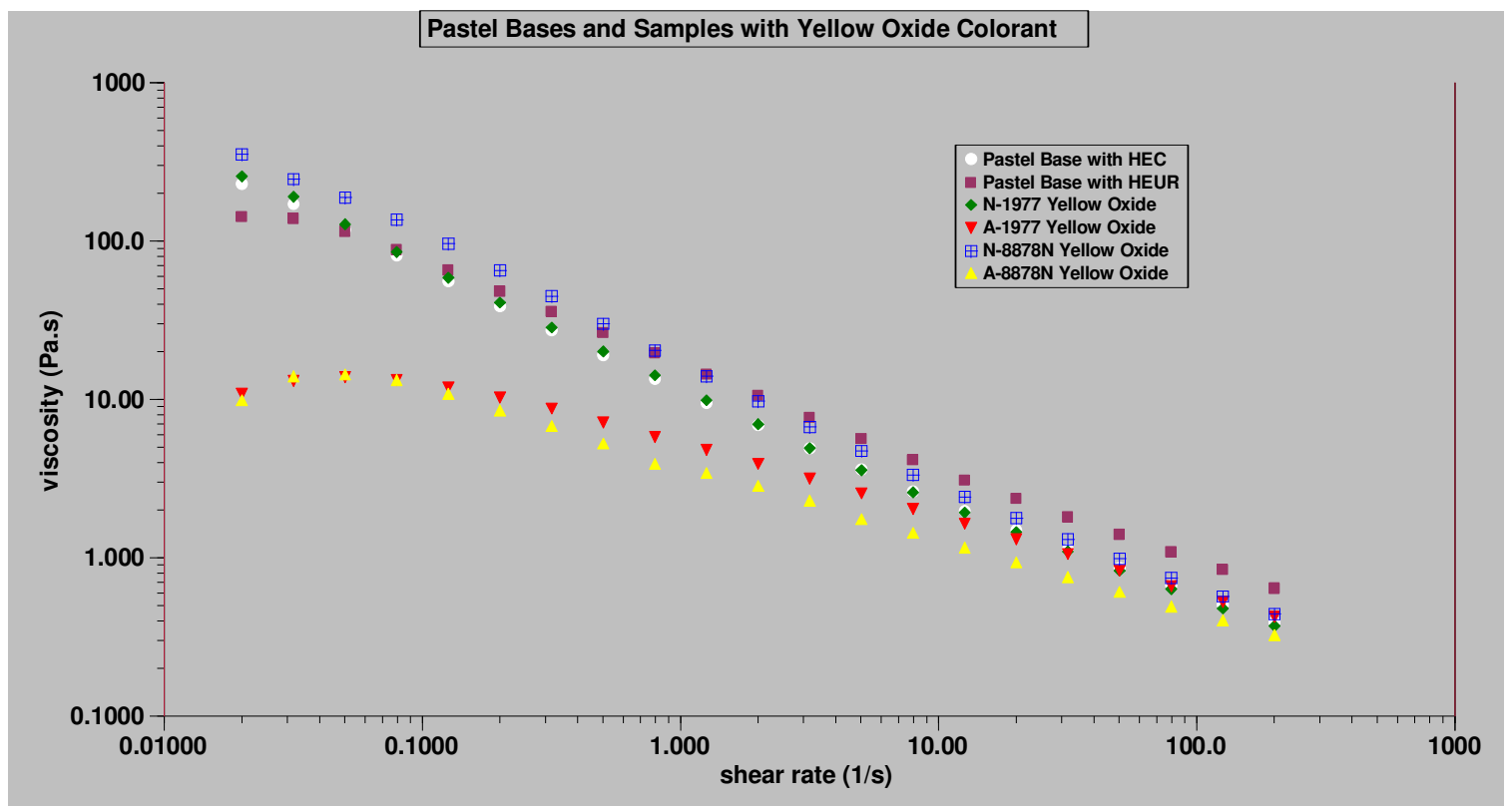

(a)

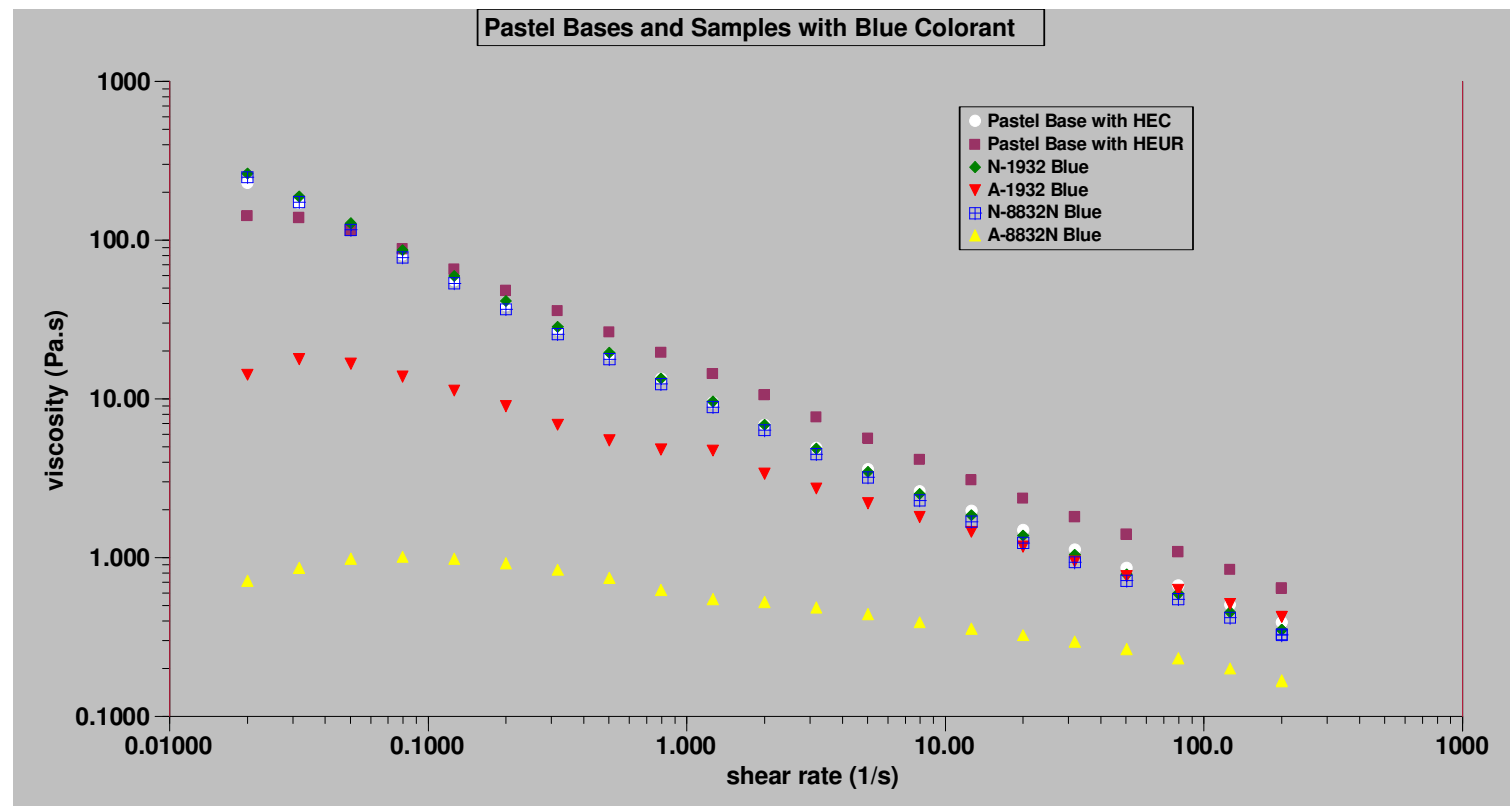

(b)

Figure 7. Viscosity profiles of: (a) yellow oxide samples and (b) blue samples 
The samples formulated with yellow oxide and blue colorants are examples of the general trend seen in the results; which was that the HEUR-thickened samples had significantly lower viscosities after colorant addition compared to the HEC-thickened samples. The excess amount of surfactants in the samples displaced the associative thickener particles from the latex surfaces and prevented thickener interparticle interactions. Without the network structure imparted by the associative thickeners, the viscosity of the system is as if there were no thickeners present.

Typically in a colloidal suspension, the forces of attraction holding the particles together are broken apart with increasing strain. The almost linear nature of the viscous modulus in each of the HEUR-thickened samples means that the structure was not affected by the increasing strain. The associative thickener molecules do not align themselves along the direction of the shear field; they resist the force moving in the shear direction. This is why the moduli of the HEUR-thickened pastel base are almost linear.

\subsubsection{Strain Sweep of Nontinted Bases}

The strain sweep profiles of both bases are shown in figure 8 . This was an interesting result because it went against what was predicted (that the HEC-thickened base would be lower in modulus). The HEC-thickened base is actually higher in modulus up until about $20 \%$ strain, despite the fact that the viscosity is actually lower than the HEUR-thickened base. This is because the HEC-thickened base is sterically crowded, due to the high MW hydroxyethylcellulose chains distributed throughout the system. Chain entanglements are responsible for the high modulus at lower strains, and after $10 \%$ strain is reached, the cellulose polymers are separated and the viscous forces dominate the behavior of the system. Under shear, the thickener becomes aligned along the shear field; this is why there is such a dramatic decrease in elastic modulus. The network formed from the hydrophobic/hydrophilic interactions in the HEUR-thickened samples is flexible, as noted by the low modulus at lower percent strains. The chemical attraction between hydrophobic thickener and latex is the reason why the modulus is not affected that much as the 


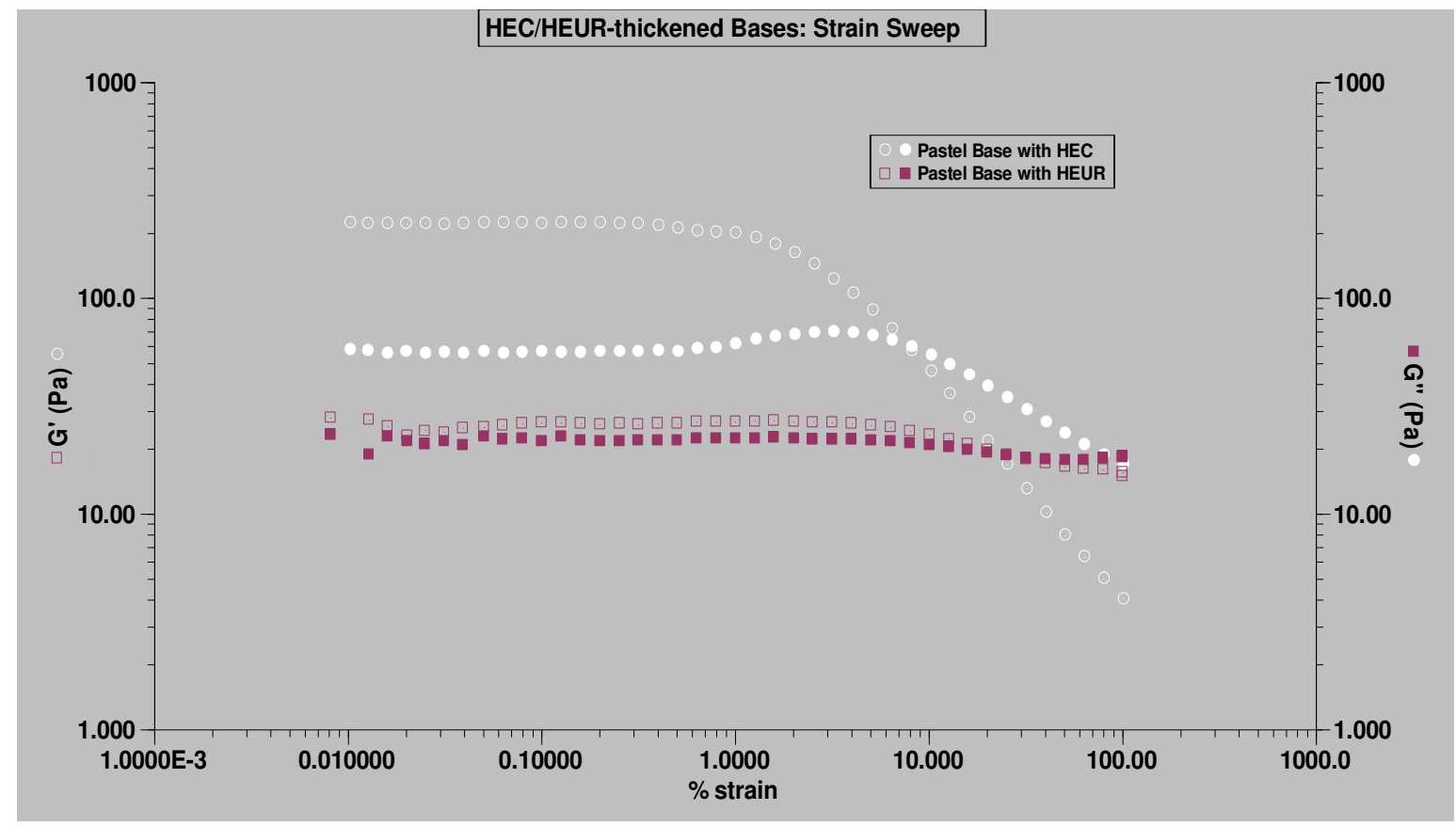

Figure 8. Strain sweep of nontinted pastel bases

3.1.5 Strain Sweep of Samples by Thickener and Colorant Series

The first thing to note is that the HEC-thickened samples formulated with the conventional lowVOC colorants ( 8800 series) exhibited similar behavior to those formulated with the zero-VOC colorants (1900 series) (Appendix 3, Figure 9). This is further evidence that non-associative thickeners do not interact chemically with the other ingredients in a given coating formulation. The profiles of the HEUR-thickened samples with the conventional low-VOC colorants were similar to those formulated with the zero-VOC colorants, but were shifted in different directions (shown in figure 9 below). One might conclude that the samples are randomly distributed below the base curve, but upon closer observation, there are two groups in the HEUR-thickened samples with the conventional 1900 series colorants. The magenta and yellow samples are similar; both moduli start off increasing with increasing strain, and then decrease and intersect each other at around 10\% strain. The rest of the samples (along with A-8821N) are the only HEUR-thickened samples that are stable toward separation (before 10\% strain). The reason for this is that the viscous modulus is higher than the elastic modulus for each of these samples. 


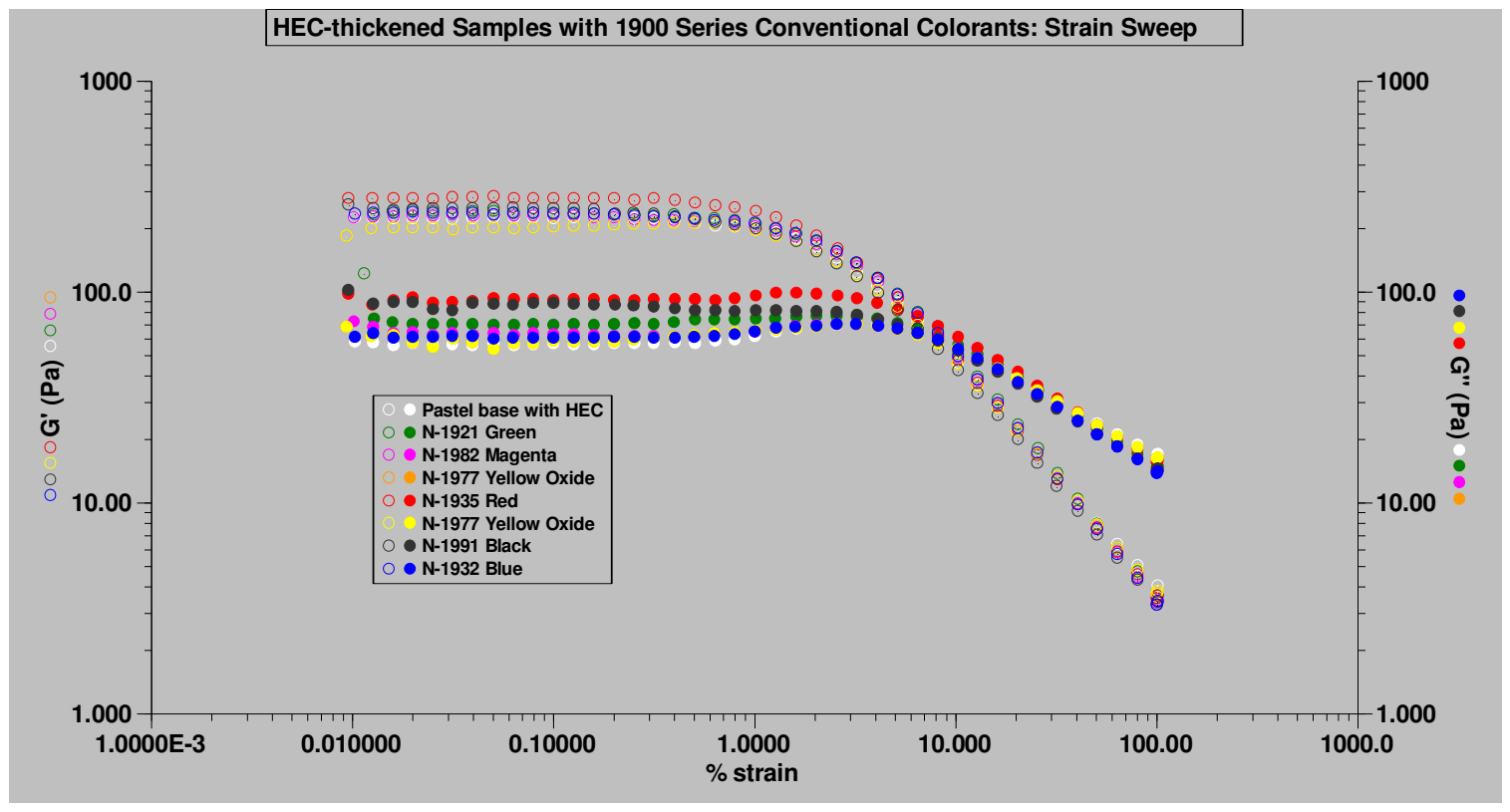

(a)

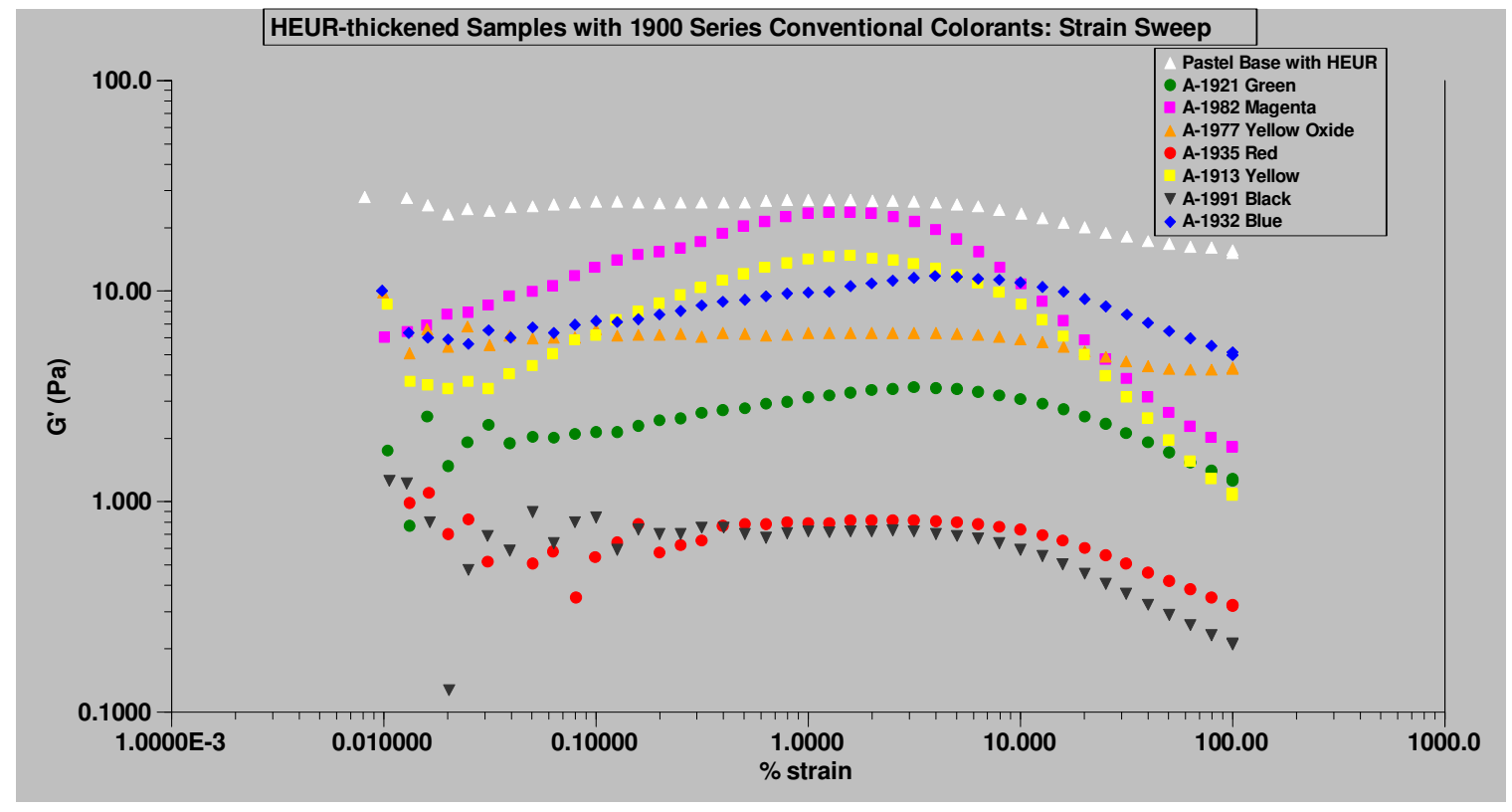

(b) 


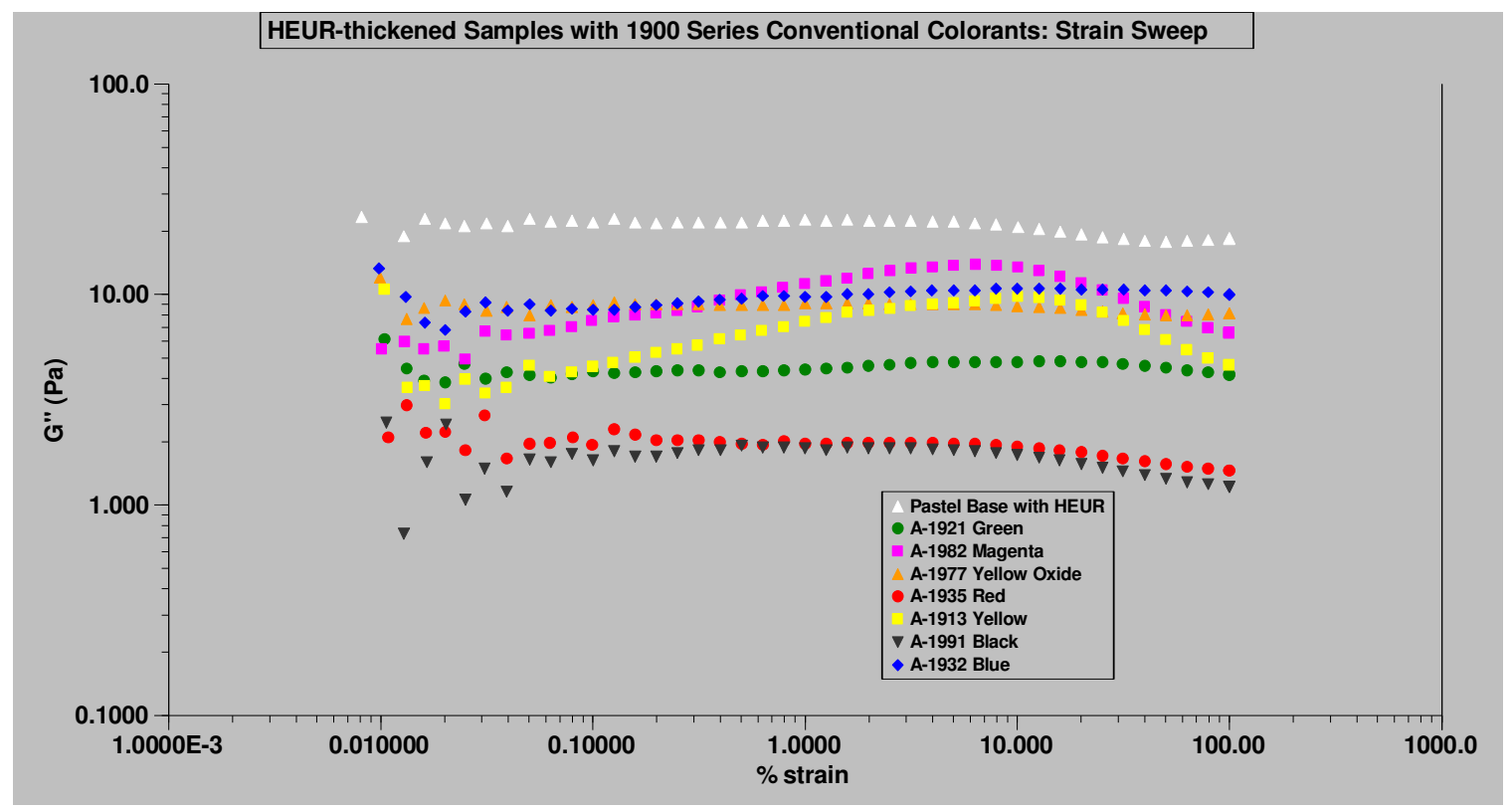

(c)

Figure 9. Strain sweeps of: (a) HEC-thickened samples with 1900 series conventional colorants,

(b) Elastic moduli of HEUR-thickened samples with 8800 series zero-VOC colorants, and (c)

Viscous moduli of HEUR-thickened samples with 8800 series zero-VOC colorants

\subsubsection{Strain Sweep of Samples by Thickener and Color}

The curves in the profiles of the HEC-thickened samples were all similar to the original

base formulation, and there is not much more that can be added to the discussion. For the HEUR-

thickened samples however, separating the samples by color makes it possible to examine the characteristic behavior imparted by each colorant. The simple fact that both G' and G" curves decreased upon addition of colorant demonstrates the influence of excess surfactant on coating rheology. Both of the HEC and HEUR-thickened 1913 yellow samples are shown in figure 10. It can also be seen here that for all of the HEUR-thickened samples except A-8821 green, A-1982, magenta, and A-1913 yellow, the viscous modulus is higher than the elastic modulus. This is indicative of a weakly-structured system, making phase separation quite possible, and indeed it was visually observed in many of these samples. According to these results, separation should not happen at rest for the three samples mentioned above. 


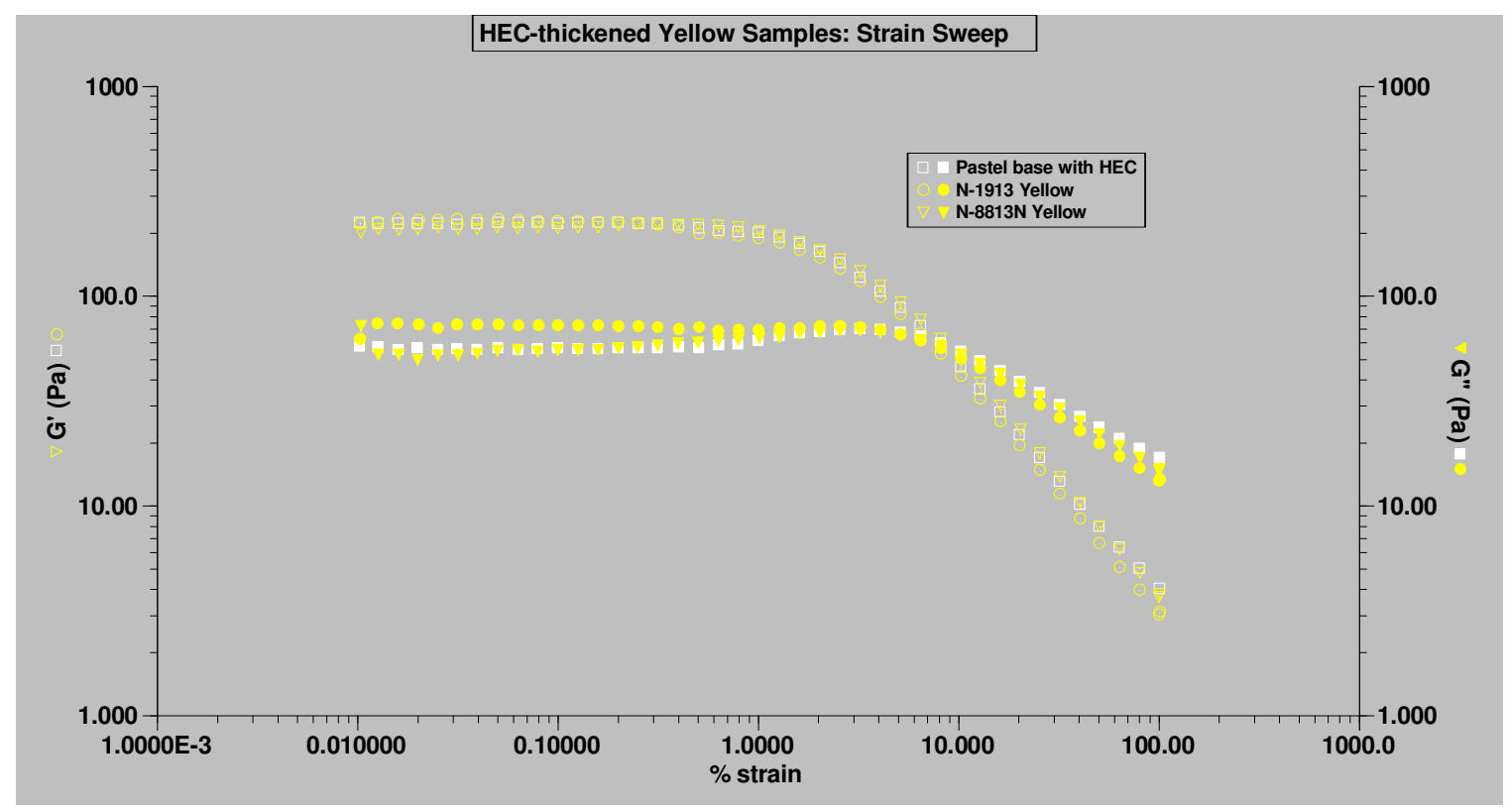

(a)

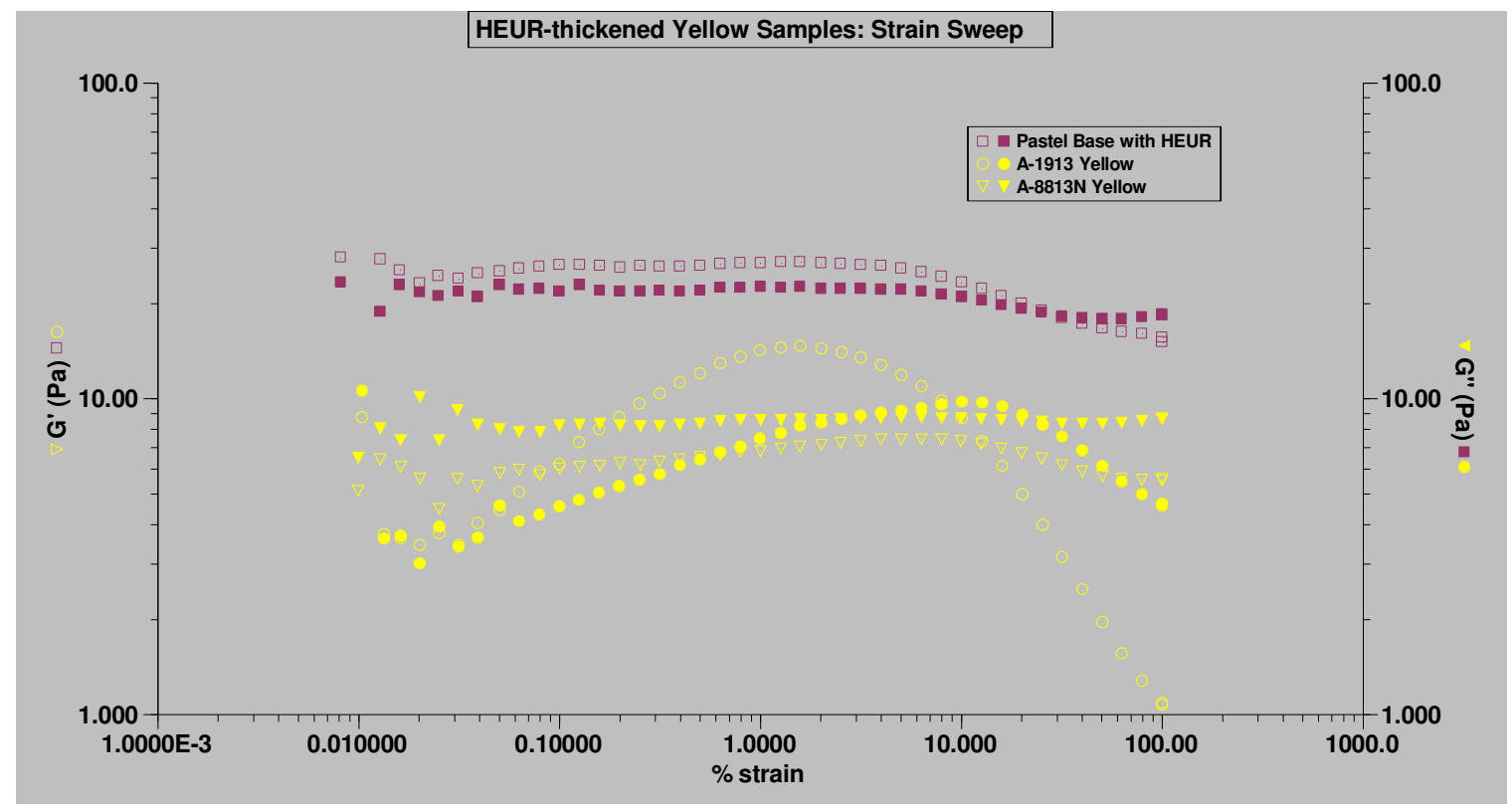

(b)

Figure 10. Strain sweep of: (a) HEC-thickened sample with 1913 yellow colorant (b) HEURthickened sample with 1913 yellow colorant

\subsubsection{Frequency Sweep of Pastel Bases}

The frequency sweep experiment results are highly impacted by the percent strains at which they were run at. However, there is still much to learn from these results (figure 11). The 
curves resemble what would be expected from a colloidal dispersion. The higher elastic modulus throughout the frequency oscillation regime indicates a more solid-like network, which is typical in colloidal systems. As the frequency of oscillation is increased, the coating dries quicker and becomes more solid-like. This is why both moduli increase as the frequency increases. Because the HEC-thickened base was run at a lower percent strain, the elastic and viscous modulus curves are higher than those of the HEUR-thickened base.

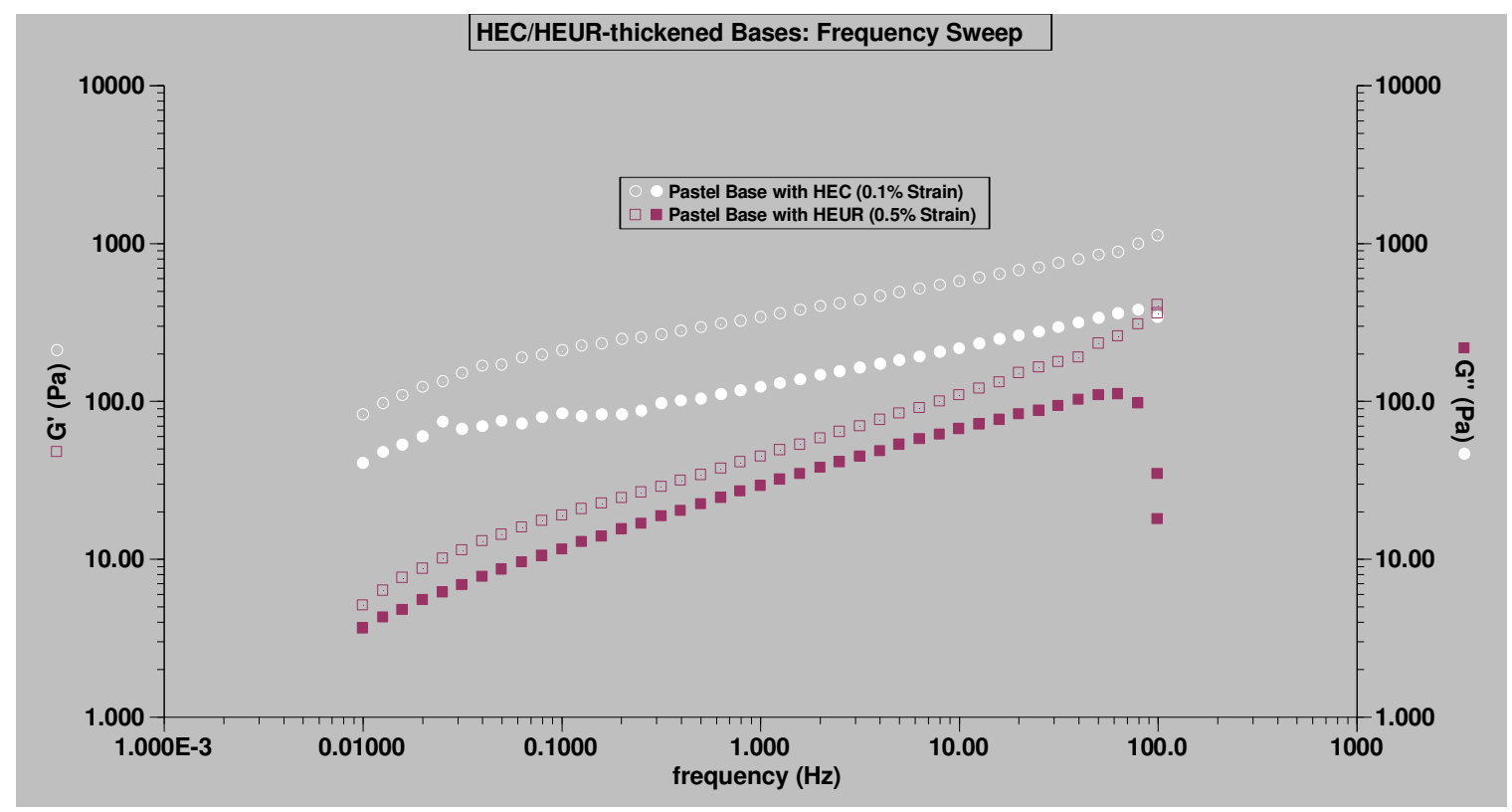

Figure 11. Frequency sweep of nontinted pastel bases

\subsubsection{Frequency Sweep of Samples by Thickener and Colorant Series}

One common theme among the HEUR-thickened samples is that despite the different percent strain the samples were run at, the samples with the 8800 series zero-VOC colorants had consistently higher elastic and viscous modulus curves (Appendix 5, Figure 12). On average, the 8800 series zero-VOC colorants contain a higher percentage of solids. Because this would increase the fraction of dispersed phase; this may be why those samples have higher elastic and viscous modulus curves. Currently, no explanation can be offered for the low elastic moduli of the samples N-1977 yellow oxide and N-8821N green. 


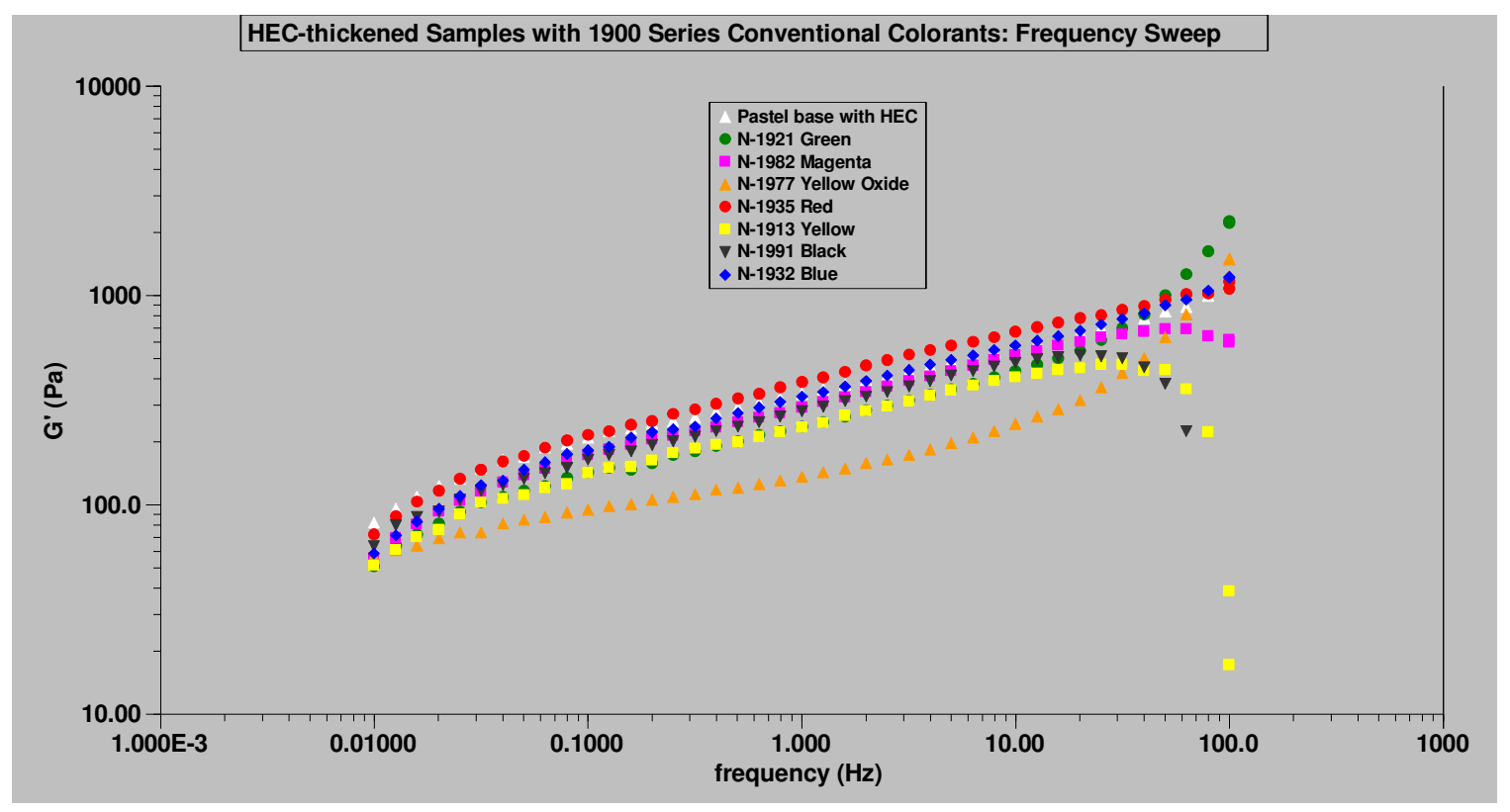

(a)

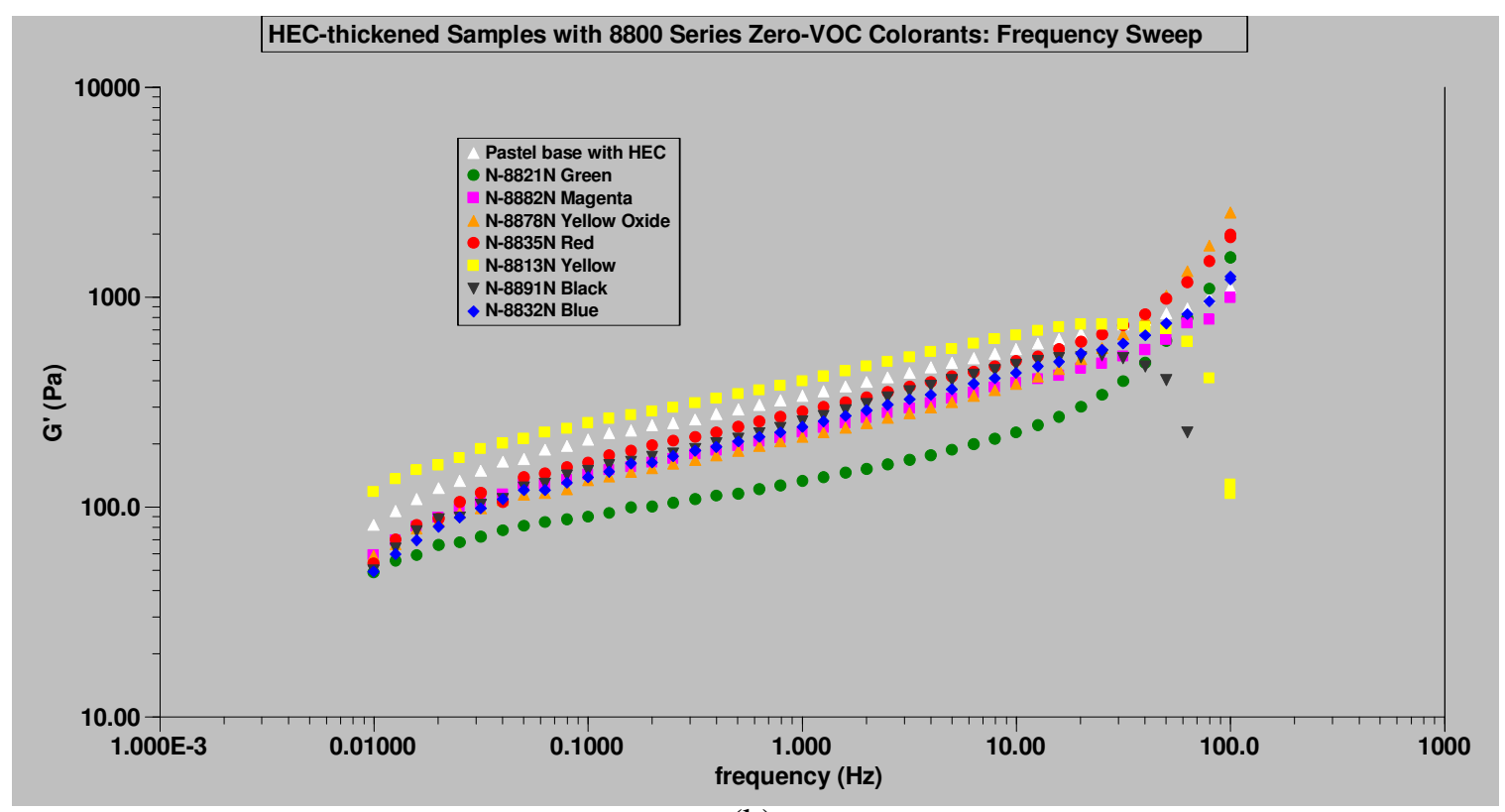

(b) 


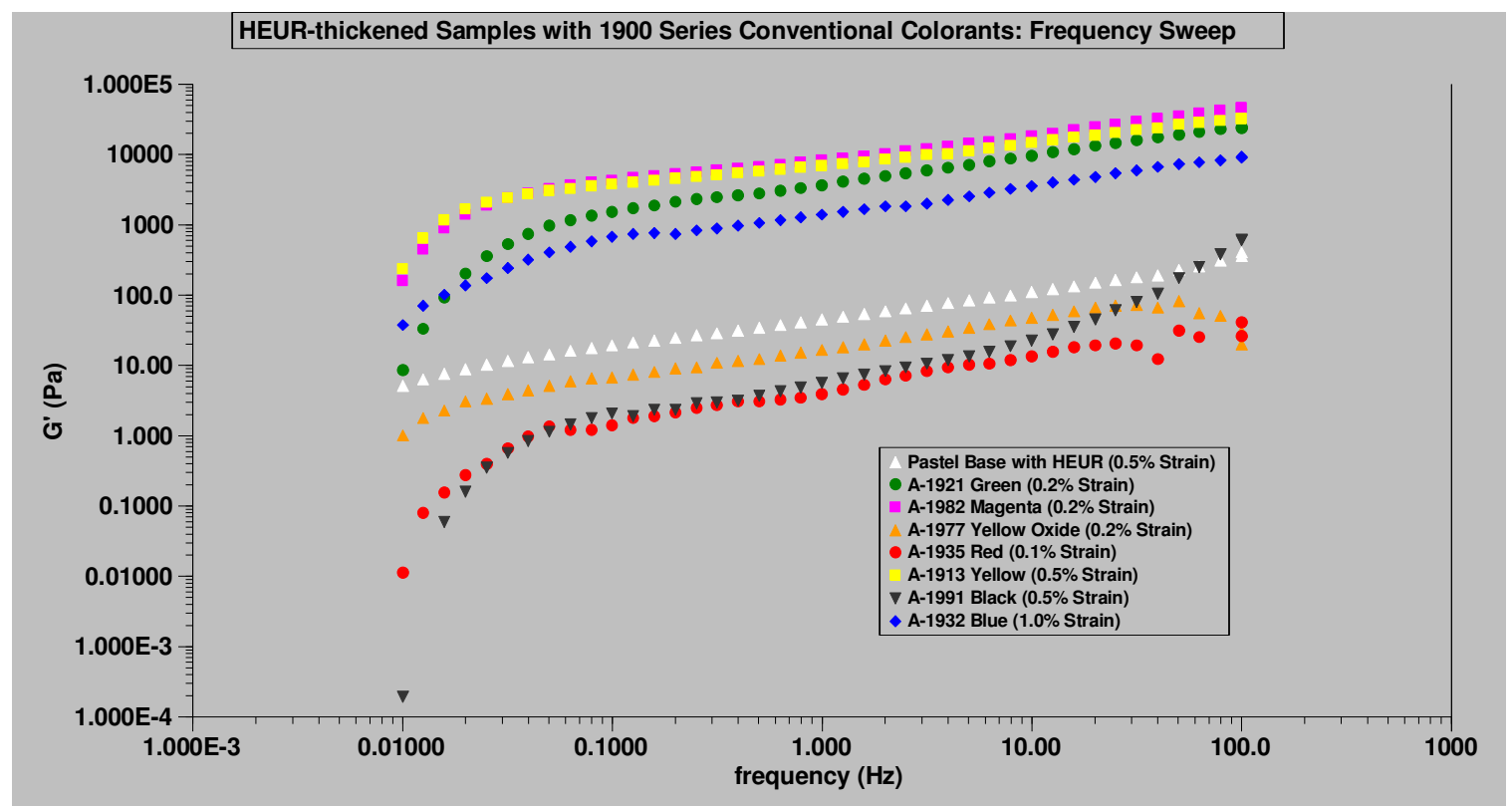

(c)

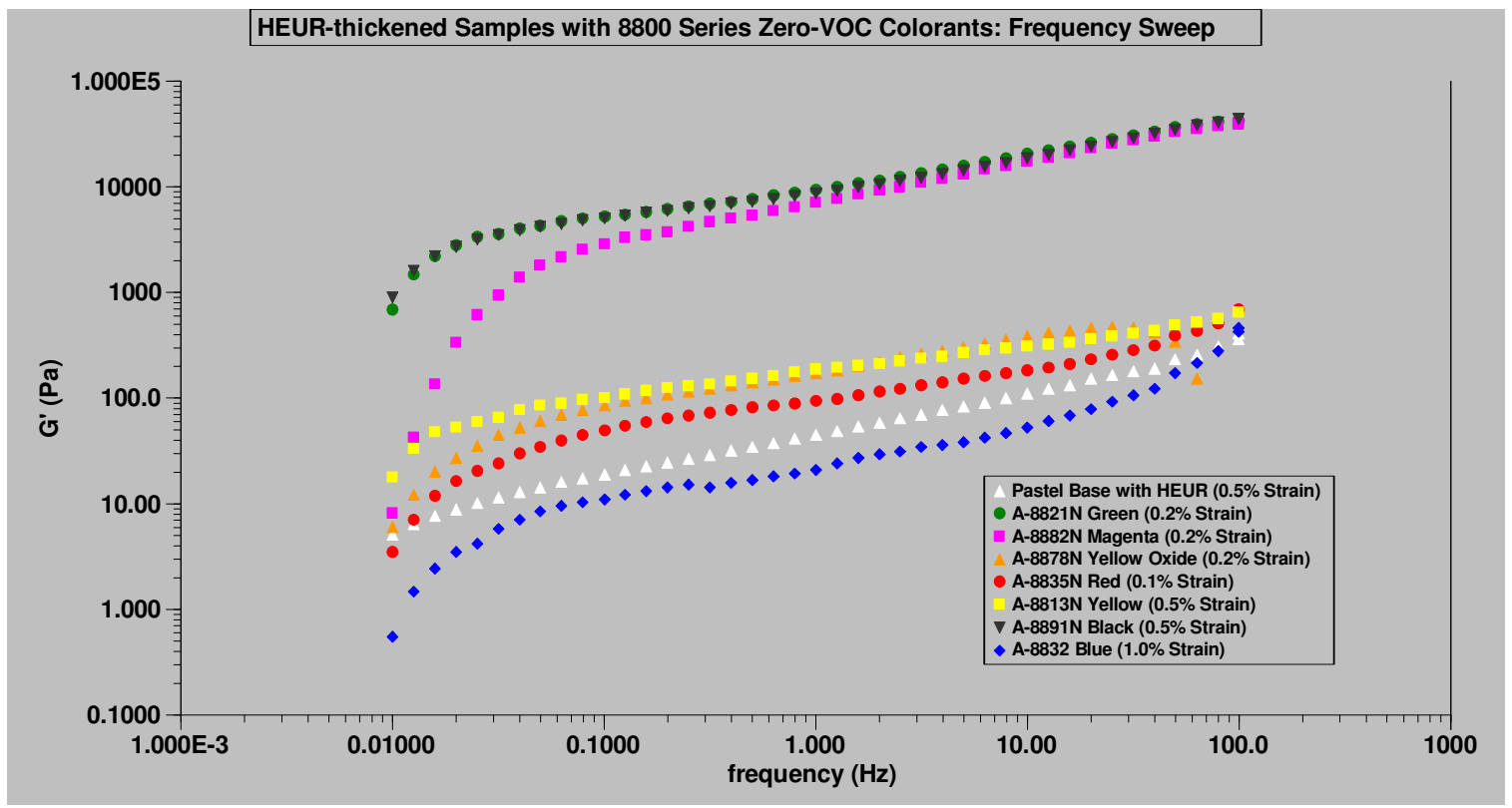

(d)

Figure 12. Frequency sweep of samples by thickener and colorant series: (a) Elastic moduli of HEC-thickened samples with 1900 series conventional colorants, (b) Elastic moduli of HECthickened samples with 8800 series zero-VOC colorants, and the same HEUR-thickened samples respectively (c), (d) 


\subsubsection{Frequency Sweep of Samples by Thickener and Color}

Except for the sample N-8813N yellow (shown in figure 13), the modulus curves of the HEC-thickened samples were all below that of the non-tinted base. It is possible that the additional surfactant provided upon addition of the colorant displaced some of the Tamol 850 polymeric dispersant from the surface of the $\mathrm{TiO}_{2}$ and/or latex particles, which possibly caused some flocculate formation within the system. Flocculation would be the reason for the small decrease in the elastic and viscous modulus curves. The strain sweep experiments were all run at the same frequency $(0.01 \mathrm{~Hz})$, however, the frequency sweeps of the HEUR-thickened samples were run at different percent strains according to the location of the linear viscoelastic region.

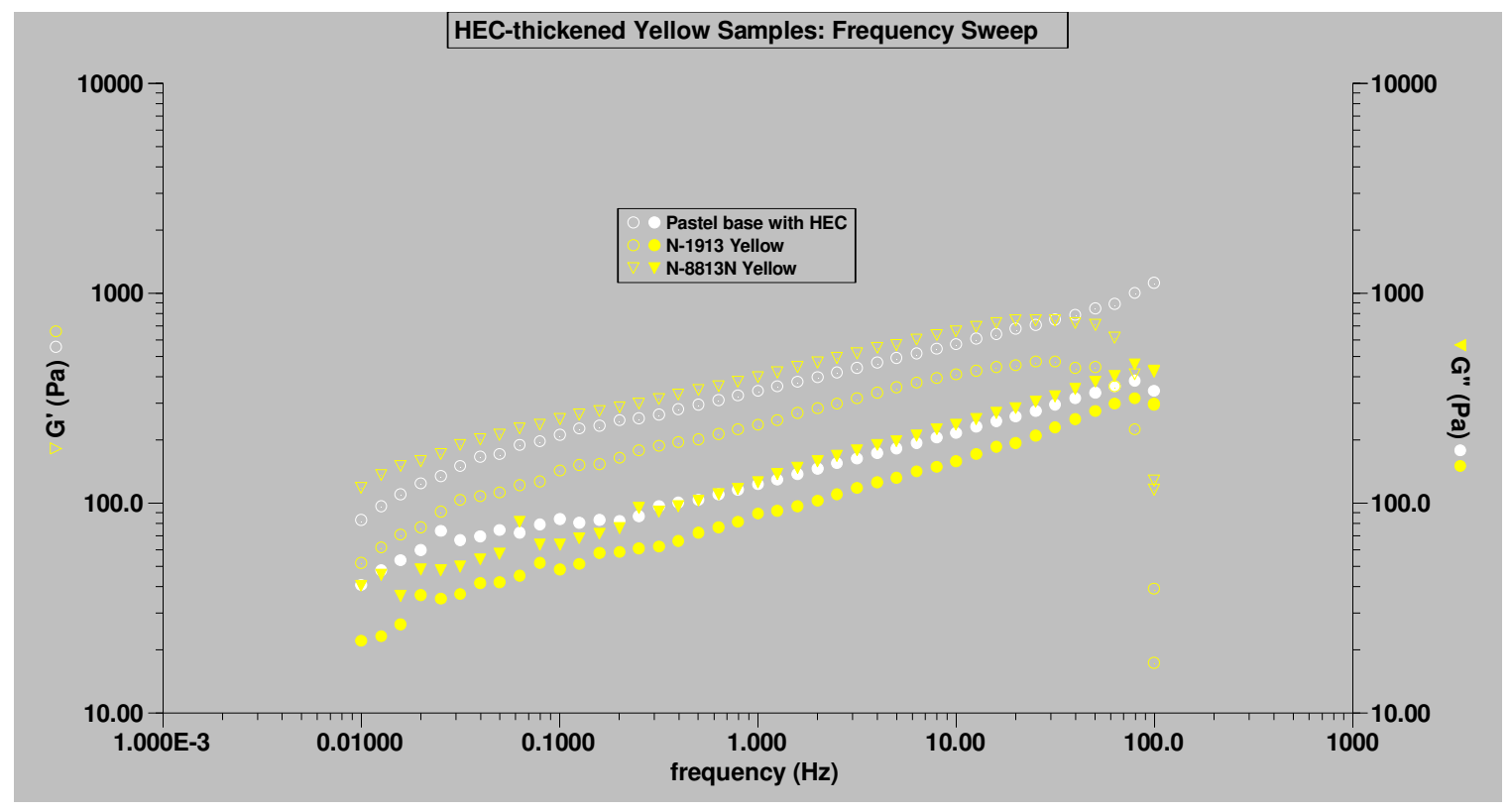

(a) 


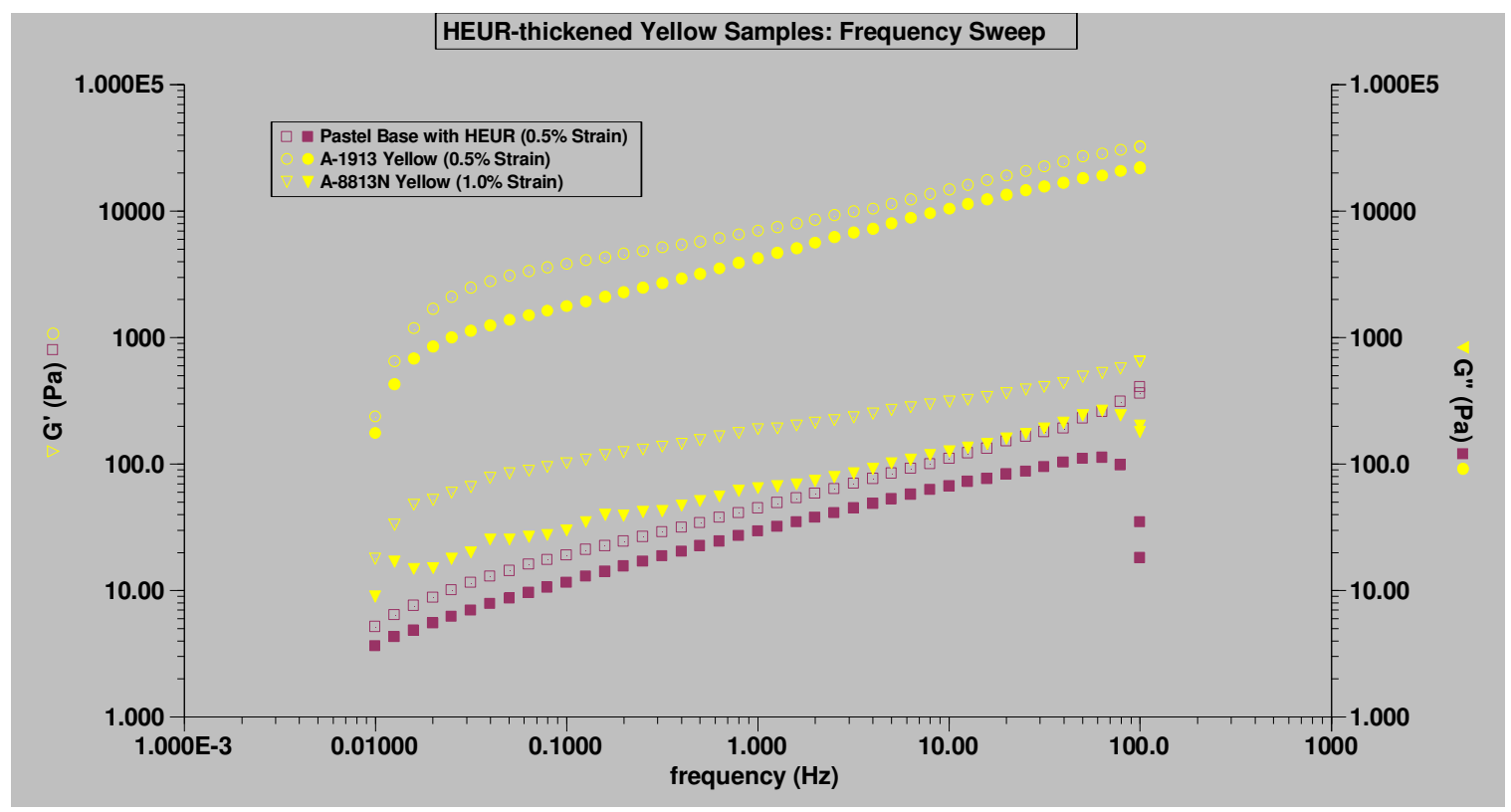

(b)

Figure 13. Frequency sweep of: (a) HEC-thickened yellow samples, (b) HEUR-thickened yellow samples

\subsubsection{0 $60^{\circ}$ and $85^{\circ}$ gloss of coated samples}

The prime factor in the determination of the gloss of a coating is pigmentation. This is quite evident here; a range of gloss values were measured for the coating samples, whose only difference was colorant and thickener. In general, the samples tinted with colorants from the zeroVOC line (8800) tended to give higher gloss (Figure 14). This result was unexpected, because latex coatings usually give lower gloss values than solventborne coatings. The reasons for this include the presence of surfactants at the surface, the presence of water-soluble dispersants and polymers that are not entirely soluble in the latex, and because latex films don't have the same opportunity to give a pigment-depleted thin layer at the surface as solventborne coatings during drying. One reason that solventborne coatings might give lower gloss values than waterborne coatings is that when solventborne coatings are dried, the film shrinks as the volatiles evaporate into the atmosphere. As this happens, pigment particles can be exposed, leaving an irregular 
surface and hence reducing gloss.

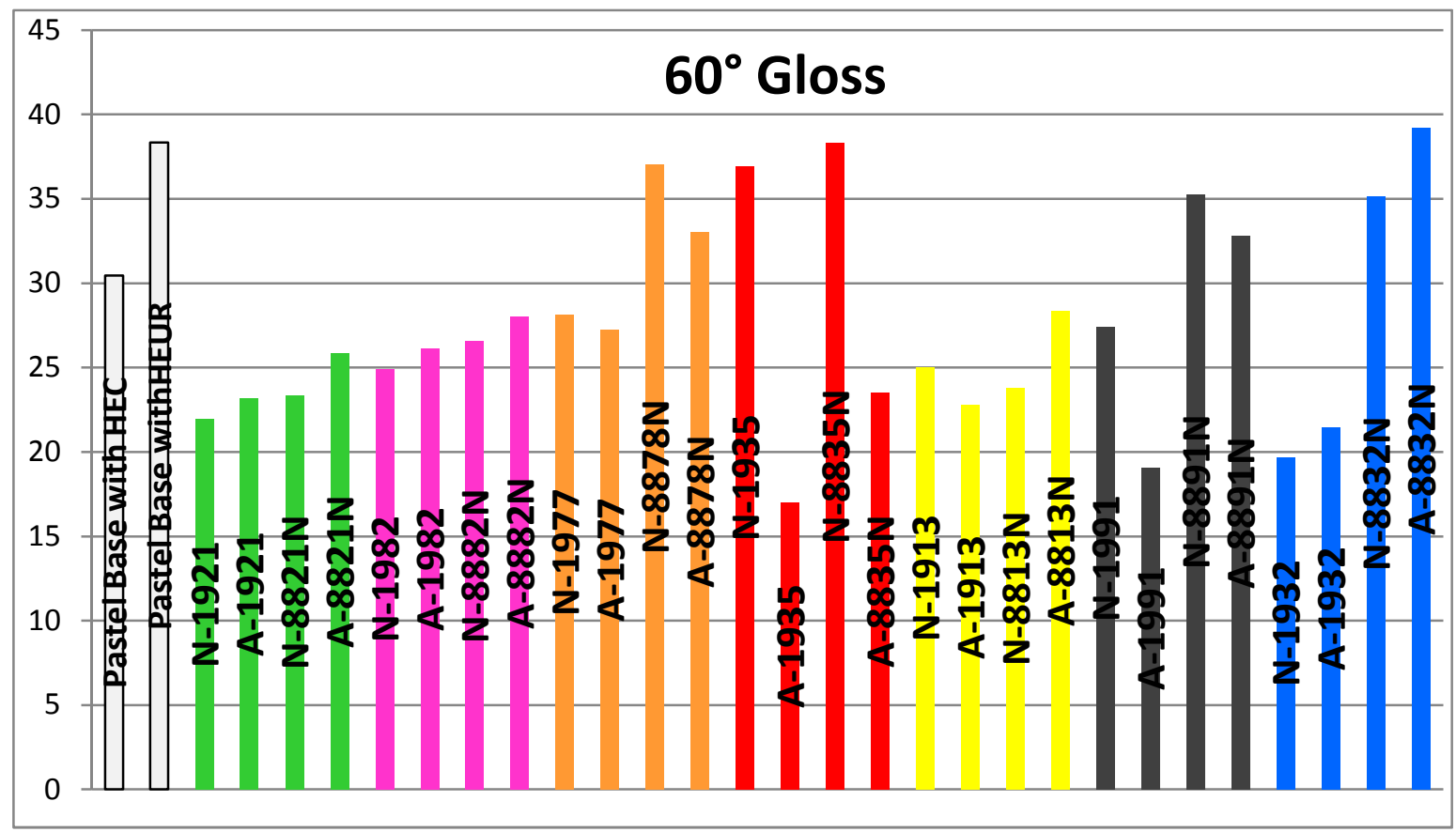

Figure $14.60^{\circ}$ gloss values of all pastel samples

The gloss values recorded at $85^{\circ}$ incidence were higher than those taken at $60^{\circ}$ because

the amount of light reflected at the specular angle increases as the angle of incidence increases

(Figure 15). The samples formulated with the HEUR thickeners gave consistently higher $85^{\circ}$

gloss values than those formulated with the HEC thickeners. This is because of the lower

viscosities of the HEUR-thickened samples. Coatings with relatively low viscosities level better

during drying than equivalent coatings of higher viscosities (the same cannot be said of the sag

properties however)! 


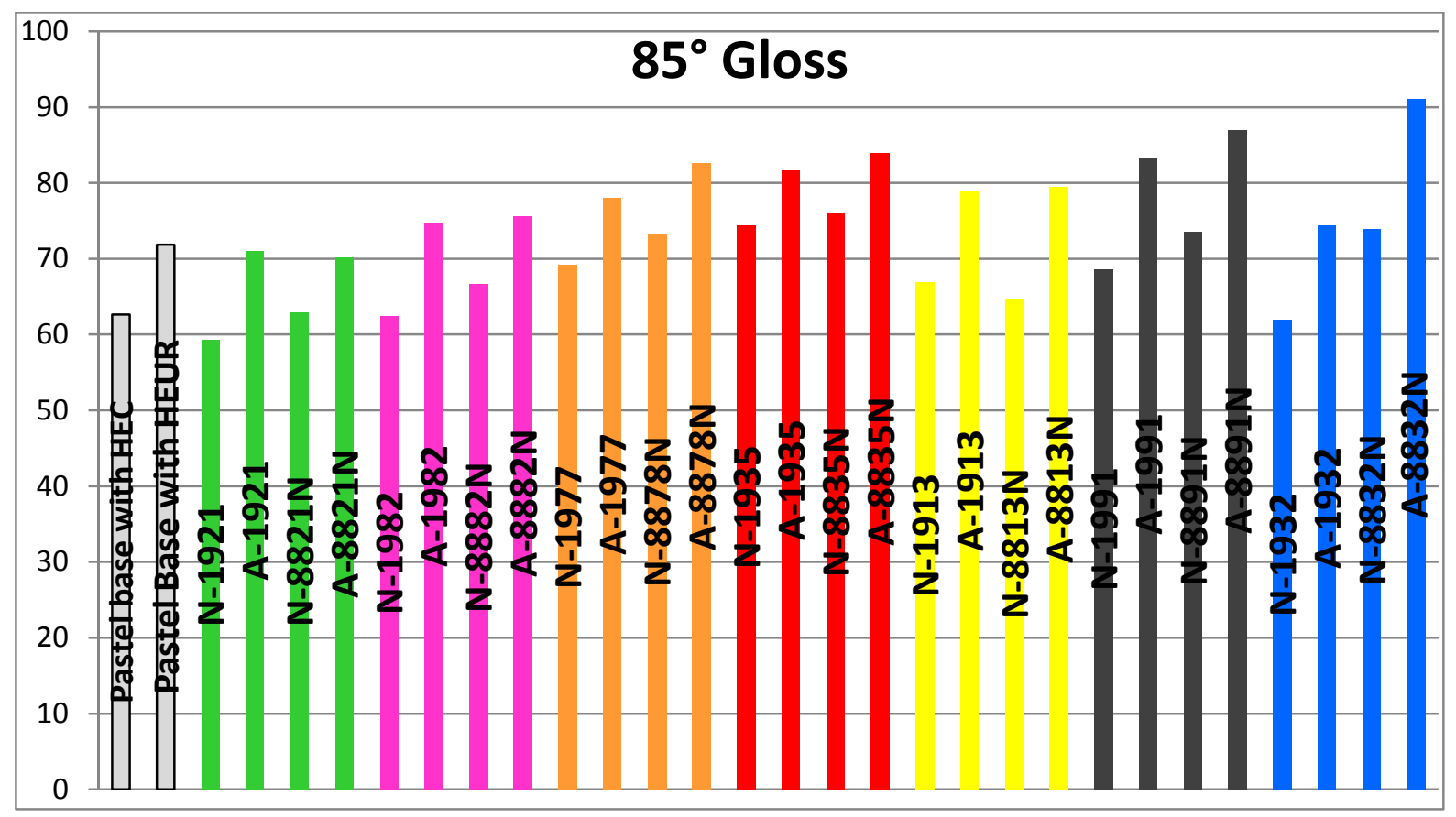

Figure $15.85^{\circ}$ gloss values of all samples

\subsubsection{Tinting Strengths of Pastel Samples}

In order to measure tint strength, the samples must be coated at a thickness sufficient to produce a contrast ratio of 0.98 . The contrast ratio of all of the samples was either above or very close to 1, which means that sufficient hiding power was obtained (Appendix 13). The results indicate that the samples with the non-associative HEC thickeners result in higher tinting strengths (Figure 16). The low tinting strength of the black samples comes from the high absorption coefficient of carbon black pigments. 


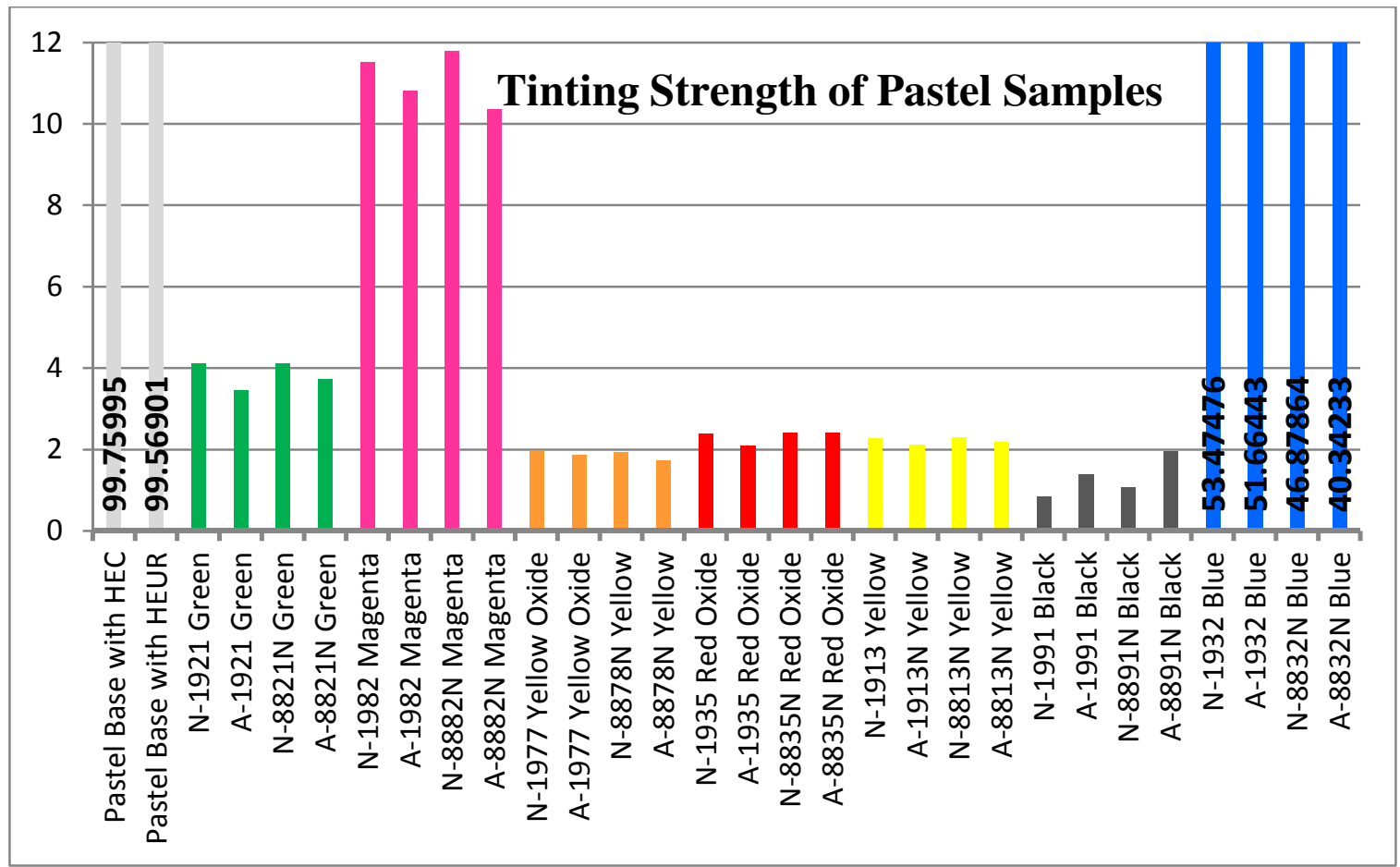

Figure 16. Tint strengths of pastel samples

\subsection{Effects of Colorant Addition on Rheology of Deeptone Bases with Associative and Non-Associative Thickeners}

\subsubsection{Viscosity Profiles of Nontinted Bases}

The deeptone bases showed similar results as the pastel bases. One of the only

differences (besides the amount of solid material in the bases) was the Stormer viscosity gap between the HEC and HEUR-thickened samples. The HEC-thickened sample had a lower Stormer viscosity, while the HEUR-thickened sample had a higher Stormer viscosity. The deeptone bases had much higher amounts of latex polymer, and so the same amount of associative thickener had a greater influence on the deeptone bases because of the higher amount of latex polymer available. The non-associative thickener chains did not have as many pigment particles to entangle around in the deeptone base vs. the pastel base, and that is why the HECthickened deeptone base has a lower viscosity. 
Table 9. Stormer viscosities and solids content of deeptone bases

\begin{tabular}{|l|c|c|c|}
\hline Sample & Initial Viscosity (KU) & 24 Hr. Viscosity (KU) & \%NV Content \\
\hline Deeptone Base with HEC & 77.8 & 78.0 & 33.2251 \\
\hline Deeptone Base with HEUR & 94.6 & 106.0 & 33.5568 \\
\hline
\end{tabular}

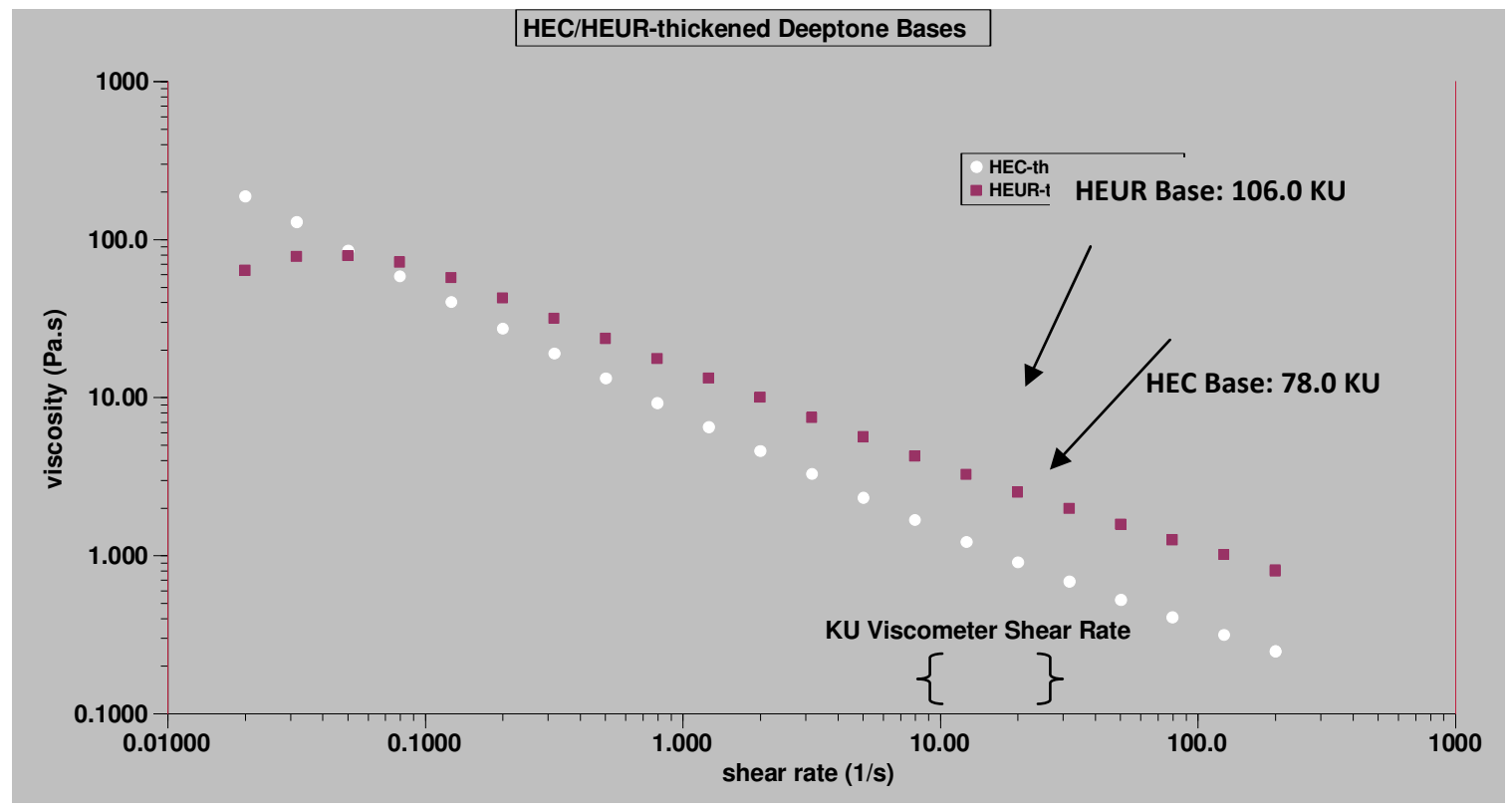

Figure 17. Viscosity profiles of deeptone bases

\subsubsection{Viscosity Profiles of Samples by Thickener and Colorant Series}

Just as with the pastel bases, the colorants seem to have no effect on the viscosity of the deeptone bases. Of the HEUR-thickened samples, both black and red oxide samples have the lowest viscosities. With the absence of $\mathrm{TiO}_{2}$ pigment, the mass of the pigment particles seems to play a greater part in influencing phase separation of the samples. This is one possible explanation, since the iron oxide red and carbon black pigments are two of the heaviest pigments in the set. 


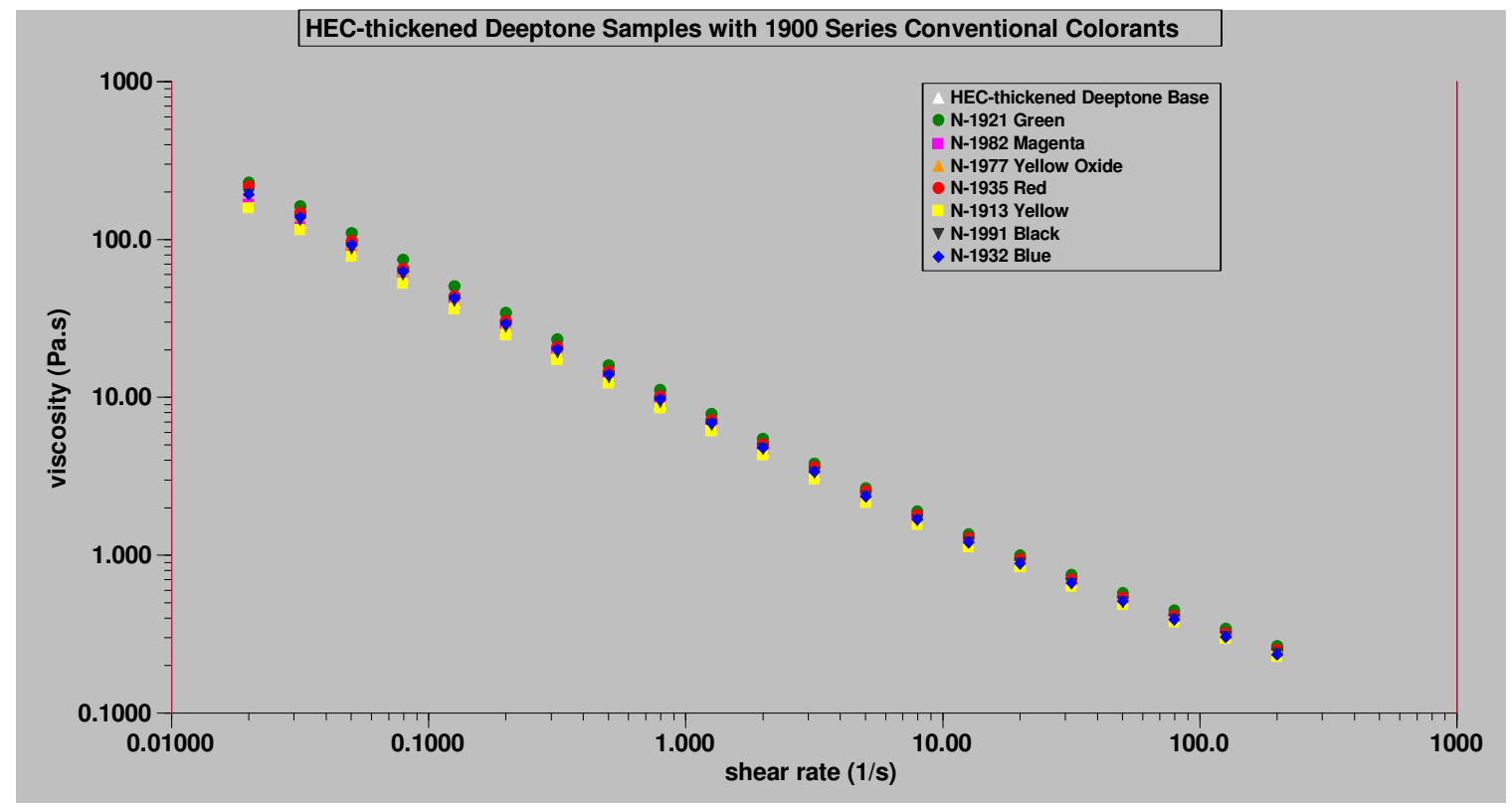

(a)

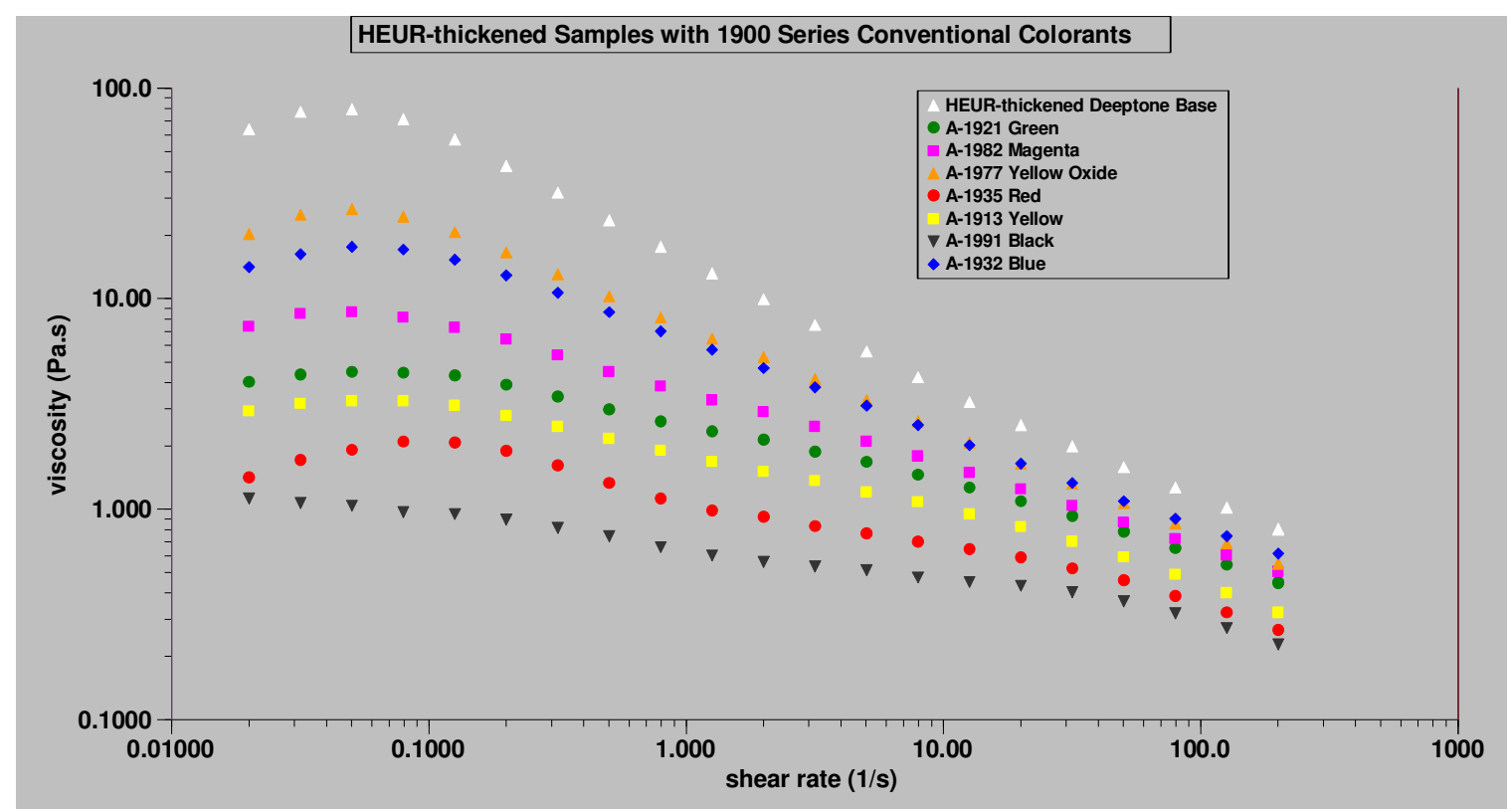

(b)

Figure 18. Viscosity profiles of: HEC-thickened samples with 1900 series conventional colorants and (b) HEUR-thickened samples with 1900 series conventional colorants

\subsubsection{Viscosity Profiles of Samples by Thickener and Color}

These graphs provide an additional view into the steadfastness of the HEC-thickened samples against colorant addition. In almost all of the HEUR-thickened samples (except for those shown in Figure 19), those with the 8800 series zero-VOC colorants had higher viscosities than 
those with the 1900 series conventional colorants. Currently, no explanation can be offered for this observation.

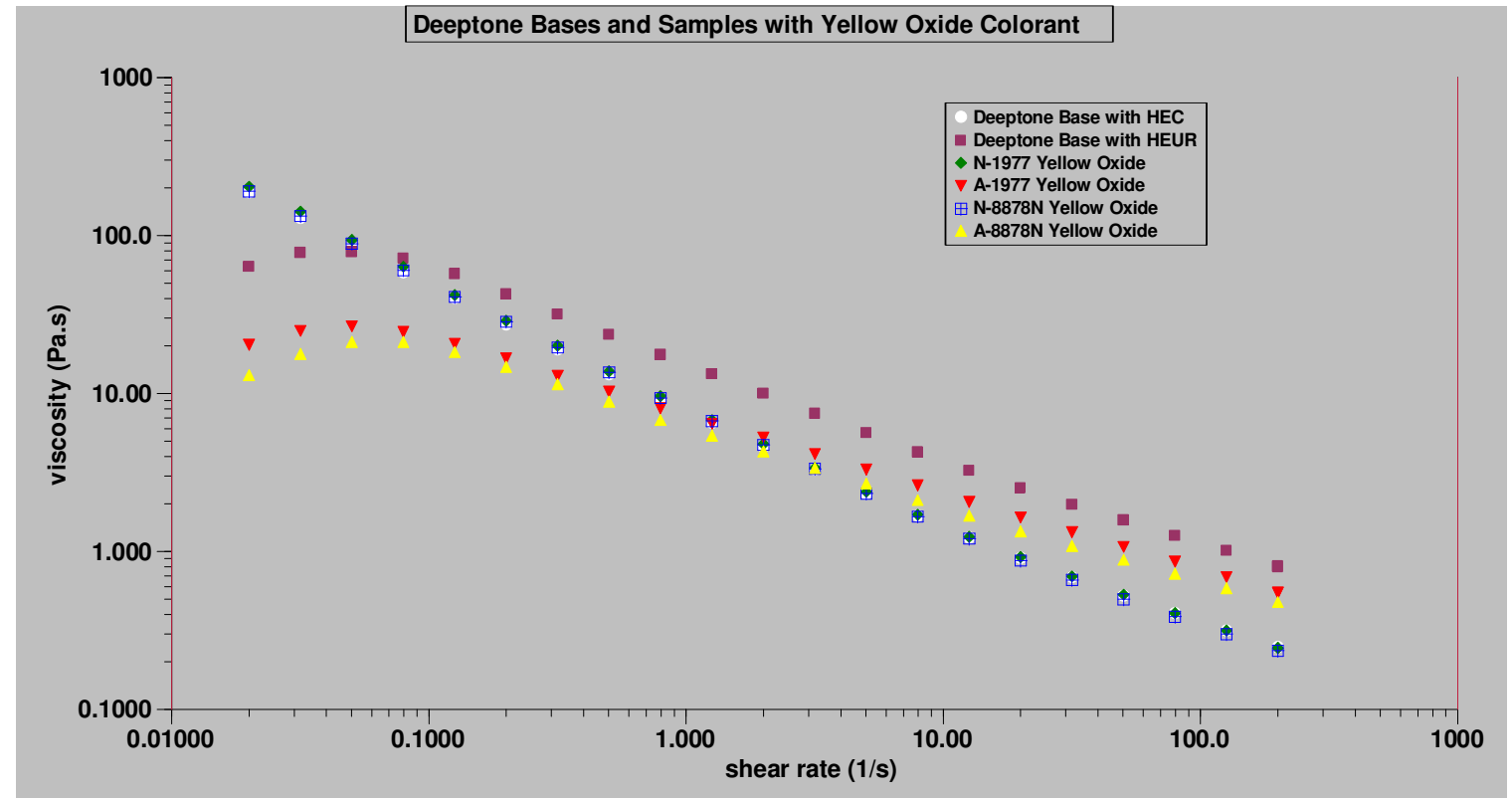

(a)

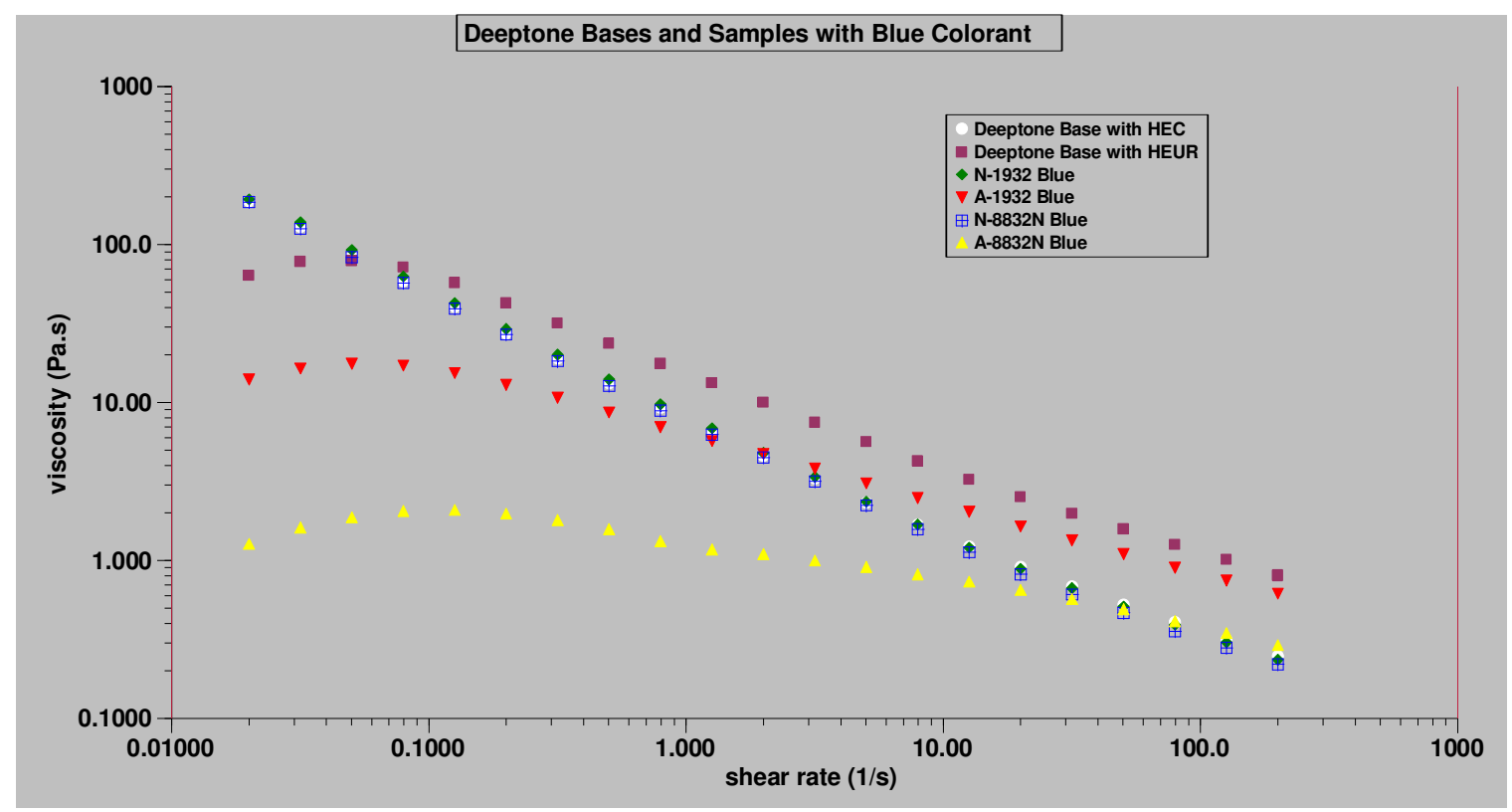

(b)

Figure 19. Viscosity profiles of: (a) deeptone bases and samples with yellow oxide colorant and (b) deeptone bases and samples with blue colorant (only two sets of samples where A-red is higher than A-yellow) 


\subsubsection{Strain Sweep of Nontinted Bases}

The results showed little difference between the pastel bases and the deeptone bases.

Again, because chain entanglements contribute the most to the modulus at low percent strains, the HEC-thickened base has higher elastic and viscous moduli.

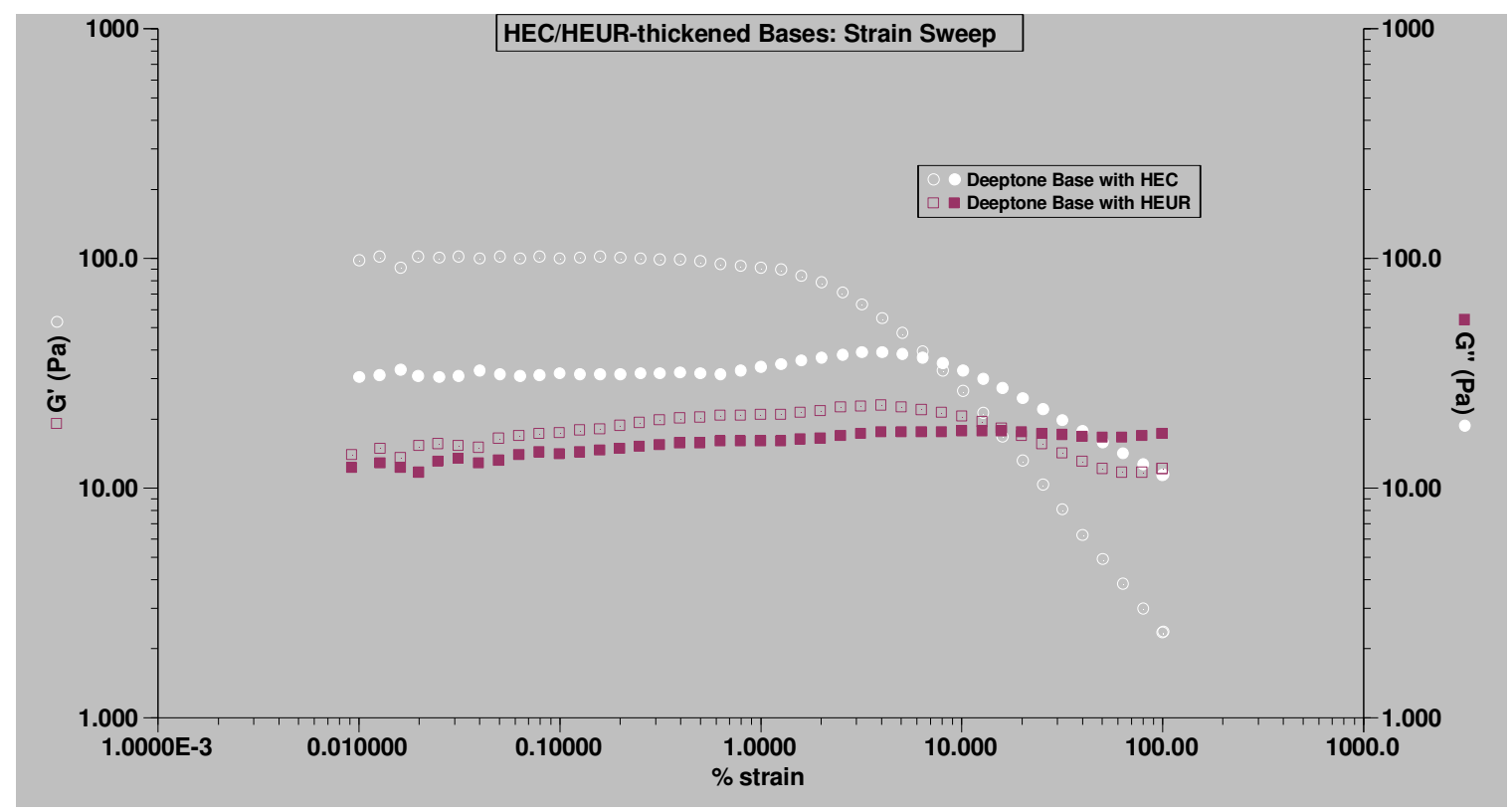

Figure 20. Strain sweep of nontinted deeptone bases

\subsubsection{Strain Sweep of Deeptone Samples by Thickener and Colorant Series}

There was little variation in between samples with HEC thickener, but not for the samples with HEUR thickener. Both elastic and viscous moduli dropped upon addition of colorant for all samples with HEUR thickener except for the one with yellow oxide colorant. Both elastic and viscous moduli of the HEUR-thickened sample with yellow oxide colorant actually increased, (albeit to a small extent) upon colorant addition. There were problems with the sister yellow oxide sample from the pastel base study, which could be the case here. Errors aside however, this was an astonishing result. Currently, no explanations can be offered for this observation. 


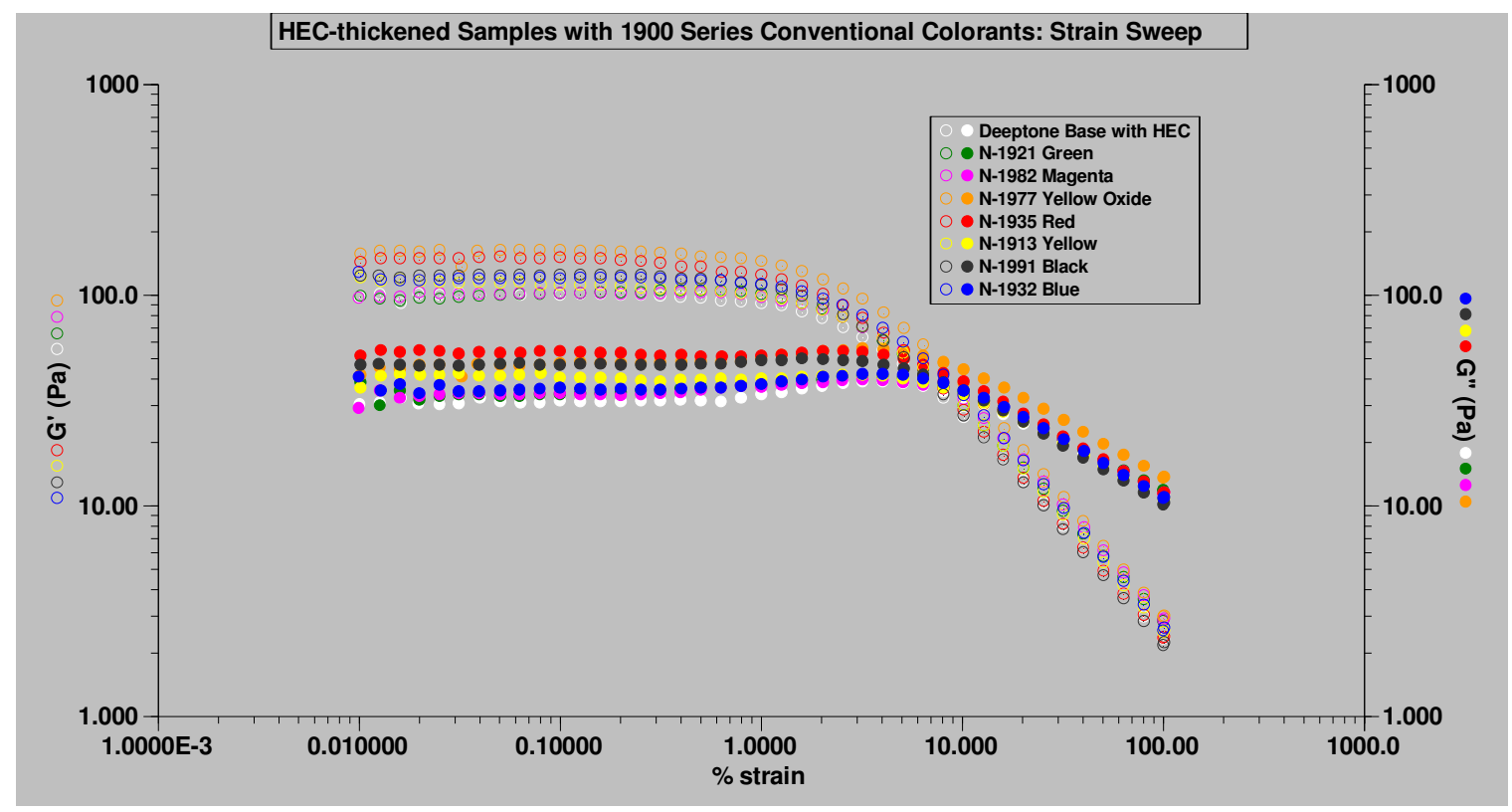

(a)

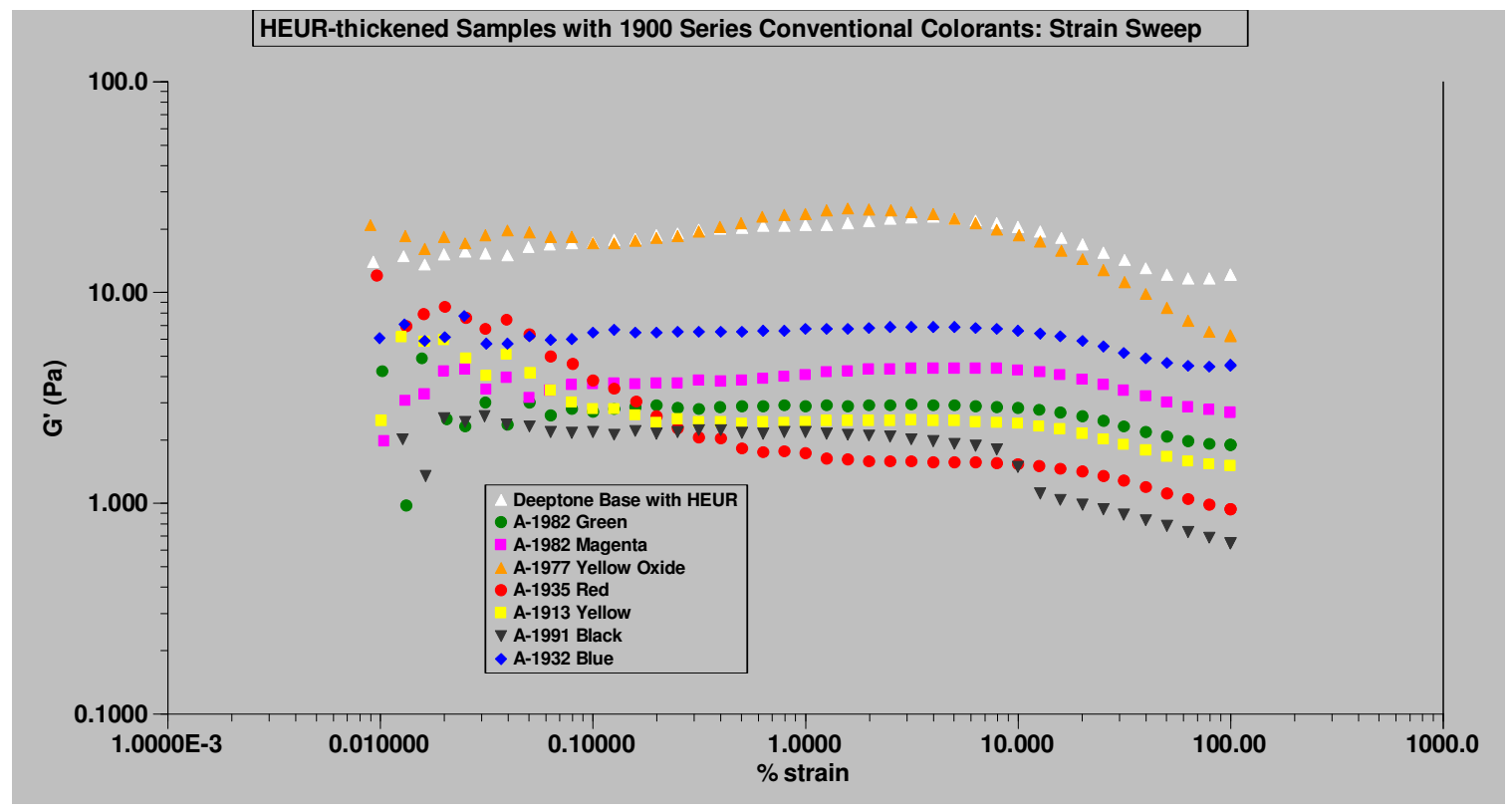

(b) 


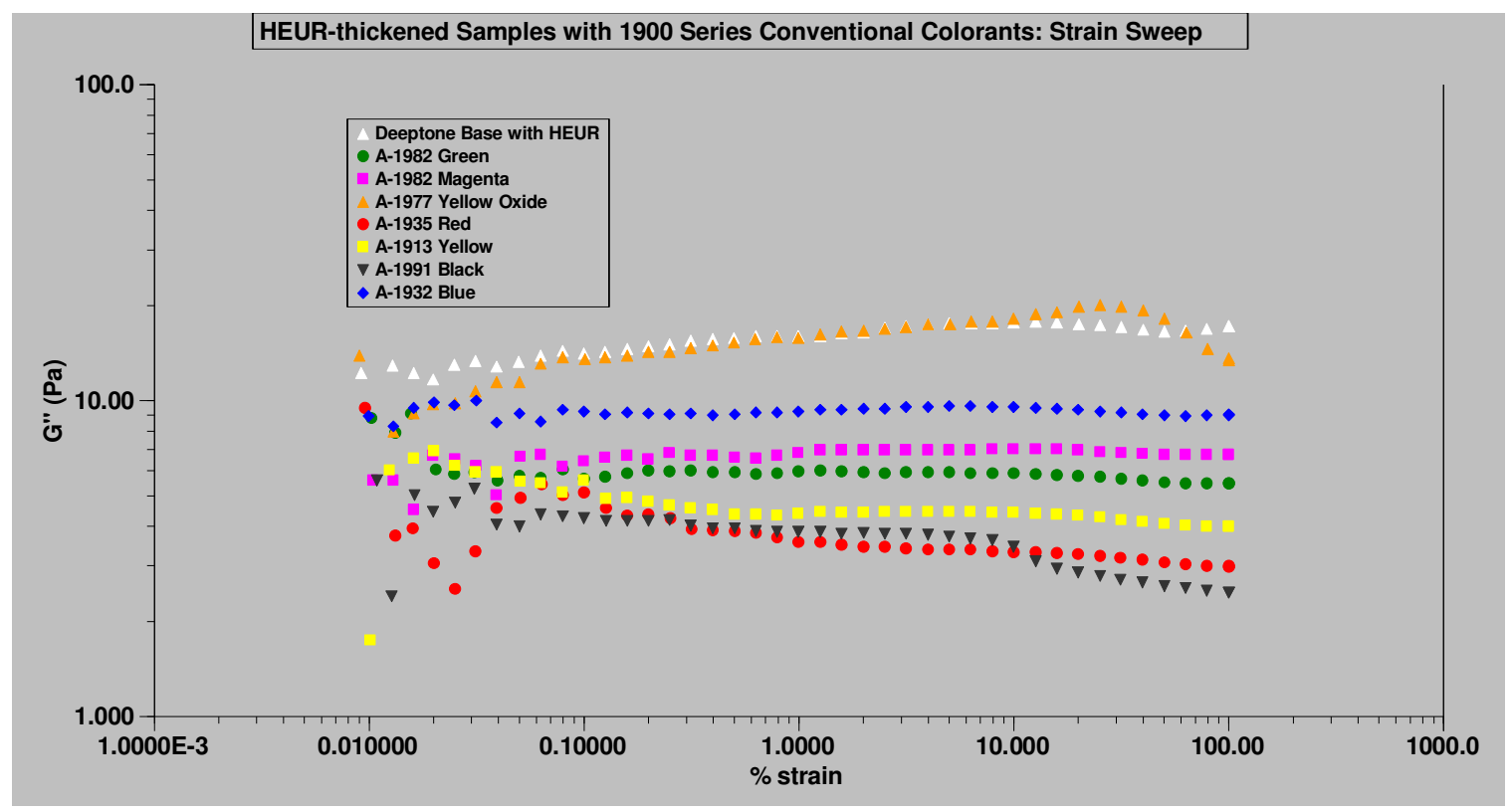

(c)

Figure 21. Strain sweep of: (a) HEC-thickened samples with 1900 series conventional colorants and elastic (b) and viscous (c) moduli of HEUR-thickened samples with 1900 series conventional colorants

\subsubsection{Strain Sweep of Samples by Thickener and Color}

These graphs provide additional insight into the peculiar result seen with the yellow oxide samples above. Both moduli increased in both HEC-thickened samples with yellow oxide, but only in the HEUR-thickened yellow oxide with zero-VOC colorant. Volume dilution effects probably caused the increase in moduli of the HEC-thickened samples. The only difference between the conventional and zero-VOC colorants is the amount of volatile organic content. The additional organic solvent somehow provided a reinforcing effect in the yellow oxide samples. There is no current explanation for this observation either, because in some of the HEURthickened samples, the moduli of the conventional colorants is higher, while in others the moduli of the zero-VOC colorant is higher. 


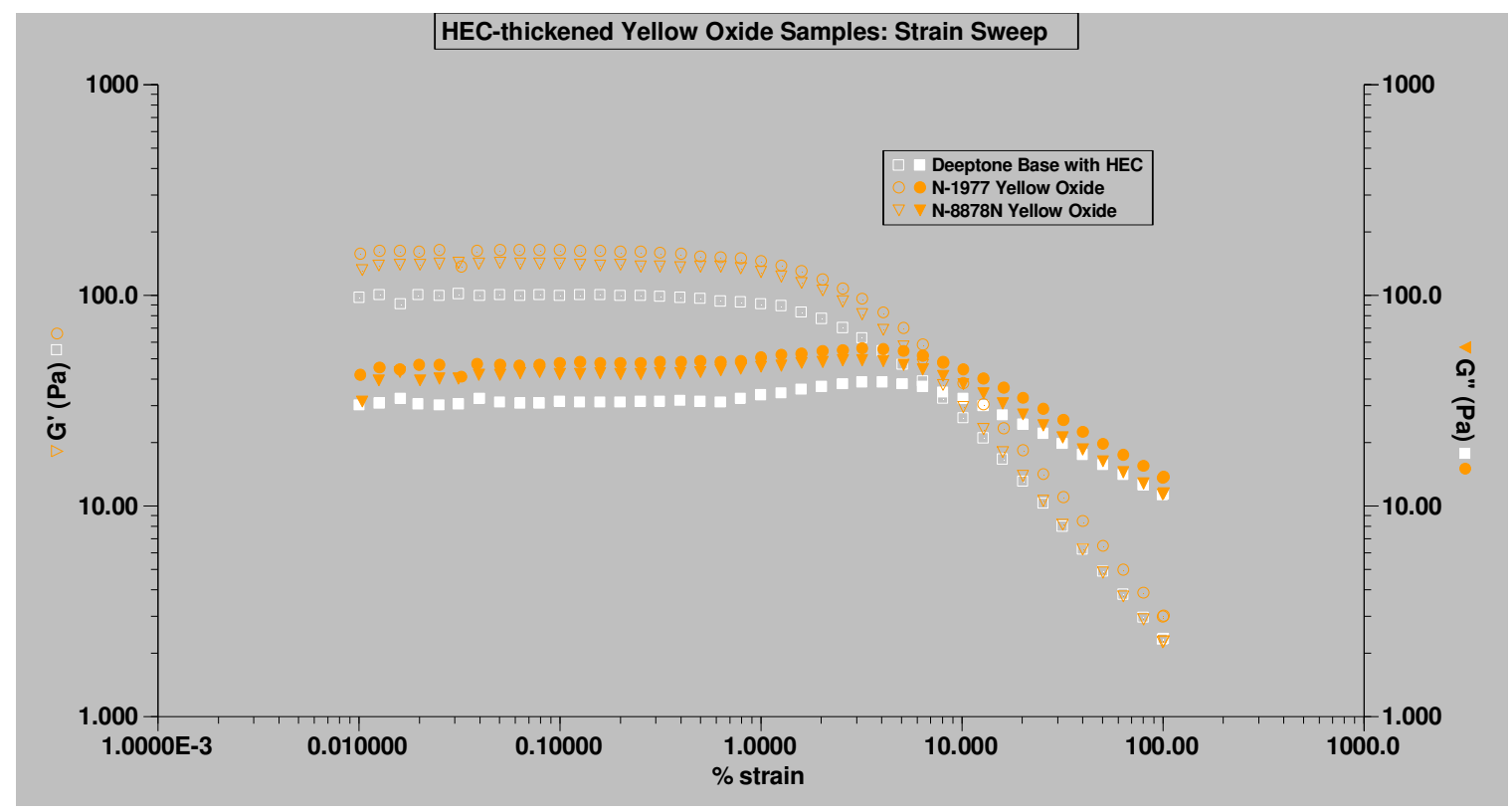

(a)

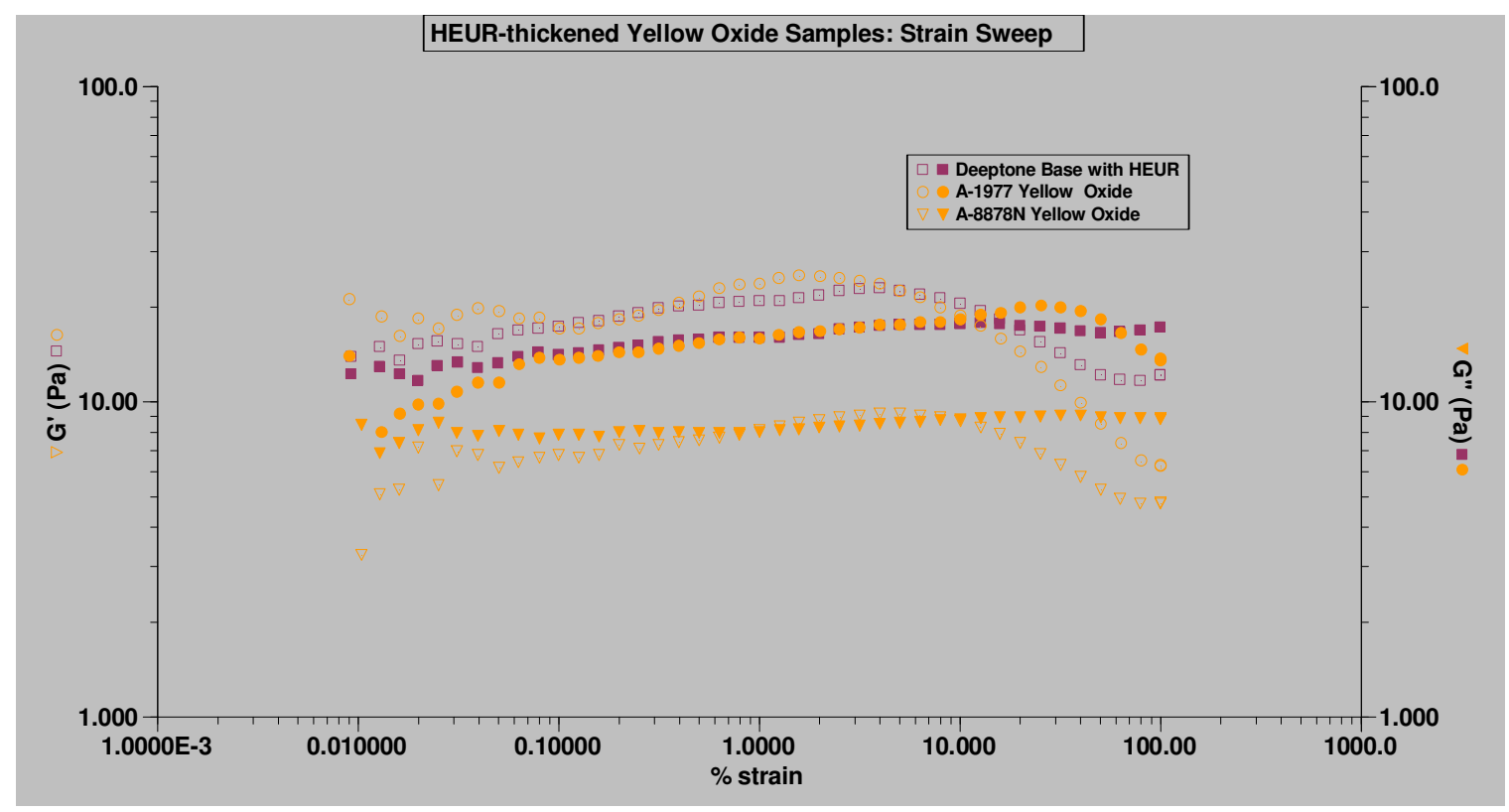

(b)

Figure 22. Strain sweep of: (a) HEC-thickened samples with yellow oxide colorant and (b) HEUR-thickened samples with yellow oxide colorant

\subsubsection{Frequency Sweep of Deeptone Bases}

The frequency sweep of the deeptone bases were exactly opposite of the pastel bases. The HEC-thickened base actually had lower moduli than the HEUR base, despite the opposite being true for the strain sweeps. Since the moduli of all of the HEUR-thickened strain sweep samples were essentially linear in nature, a common value of percent strain was chosen for all of the 
HEUR-thickened samples, $0.1 \%$ strain. This result is most likely due to the higher amount of latex polymer in the bases (vs. the pastel bases). Because the associative polymers thicken by adsorbing onto the latex particles, the presence of more latex particles would lead to more interactions, and the construction of a more durable hydrophobic network.

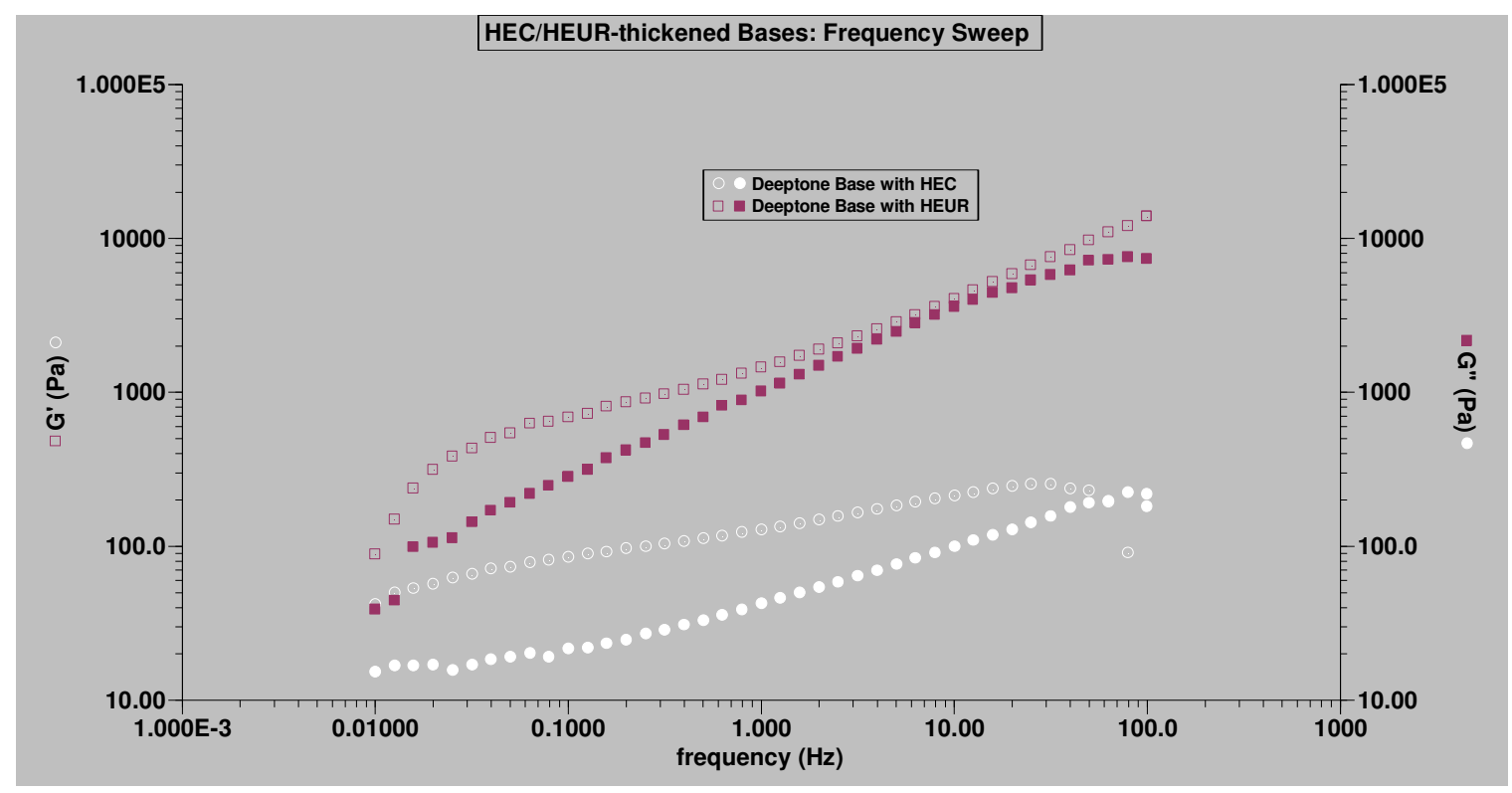

Figure 23. Frequency sweep of nontinted deeptone bases

\subsubsection{Frequency Sweep of Samples by Thickener and Colorant Series}

The moduli of the HEC-thickened samples with yellow oxide colorant are once again higher than the nontinted base. In addition, the moduli of the HEC-thickened red samples are also higher than the nontinted base. This is only true for those samples with conventional colorants however (Appendix 19). There is an interaction between the VOC components of the conventional colorants that seem to have an interaction with the dispersed red and yellow oxide pigments. This is true for the HEUR-thickened samples with yellow oxide colorants as well. One possible explanation for the HEUR-thickened samples is that the yellow oxide pigments have the smallest particle size and highest surface areas of the pigments used in this study. The majority of the surfactants and dispersants were being used to stabilize the high surface area yellow oxide particles, and only a small amount of excess surfactants displaced the associative thickeners from the latex particles. 


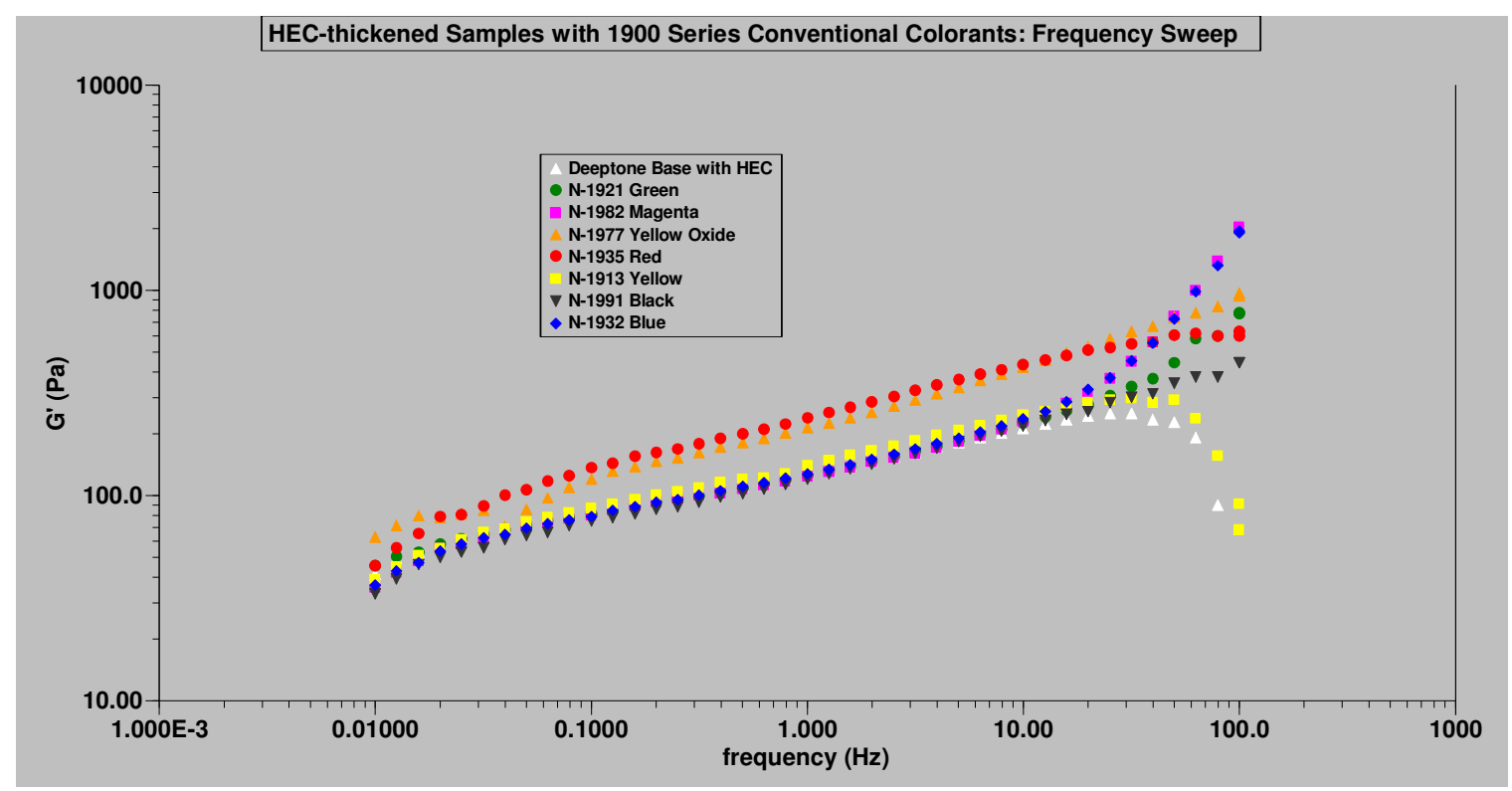

(a)

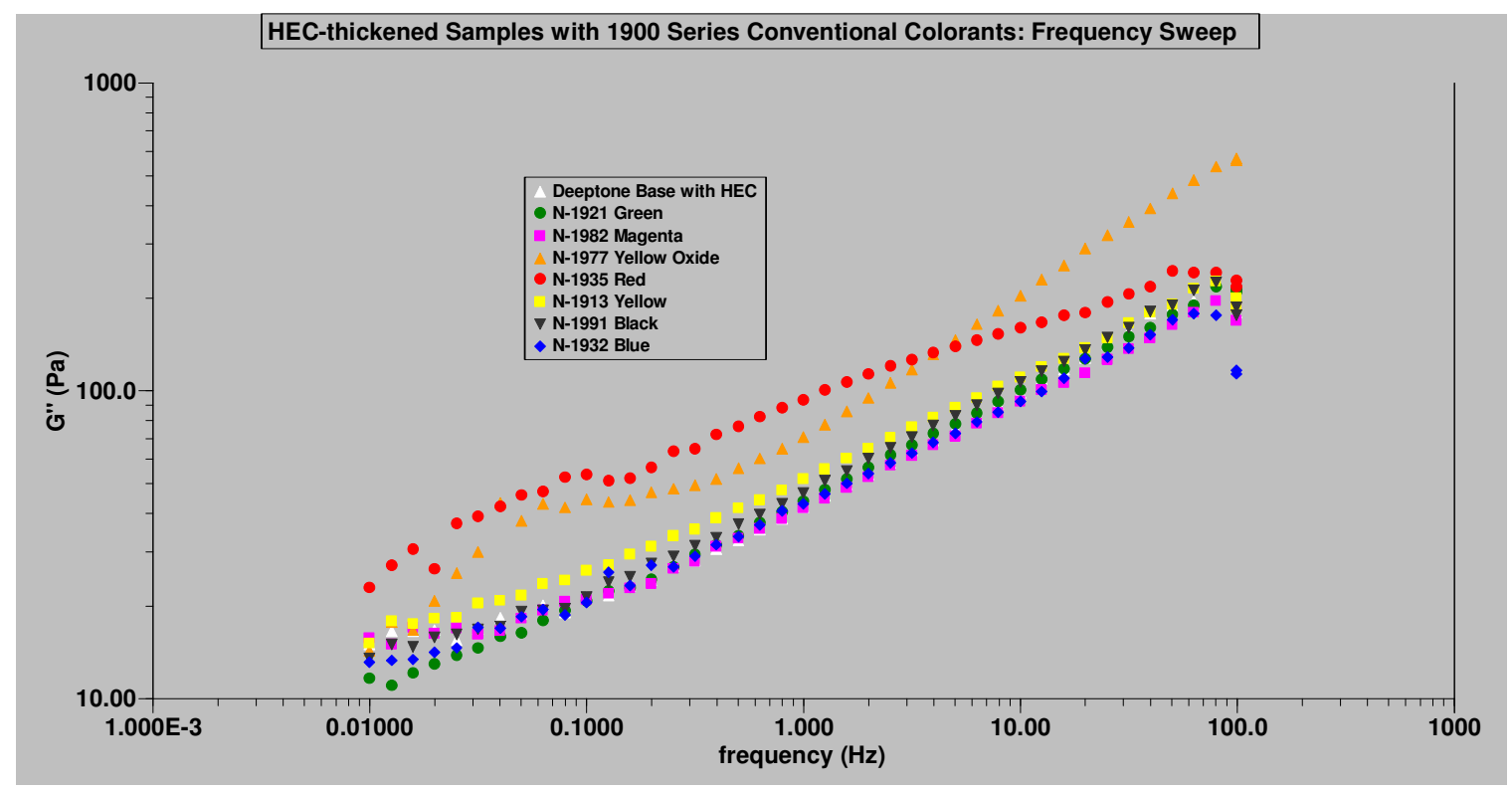

(b) 


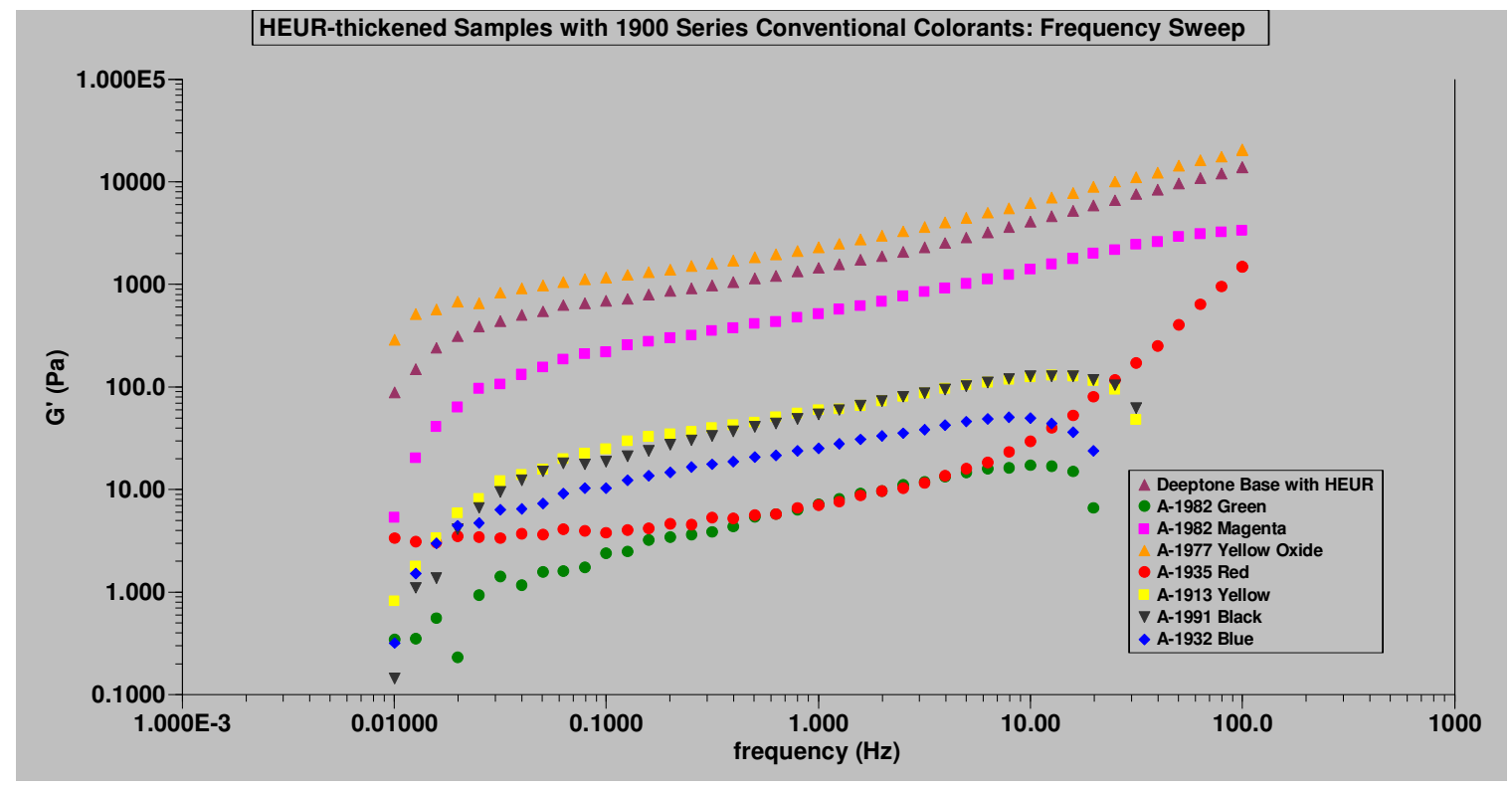

(c)

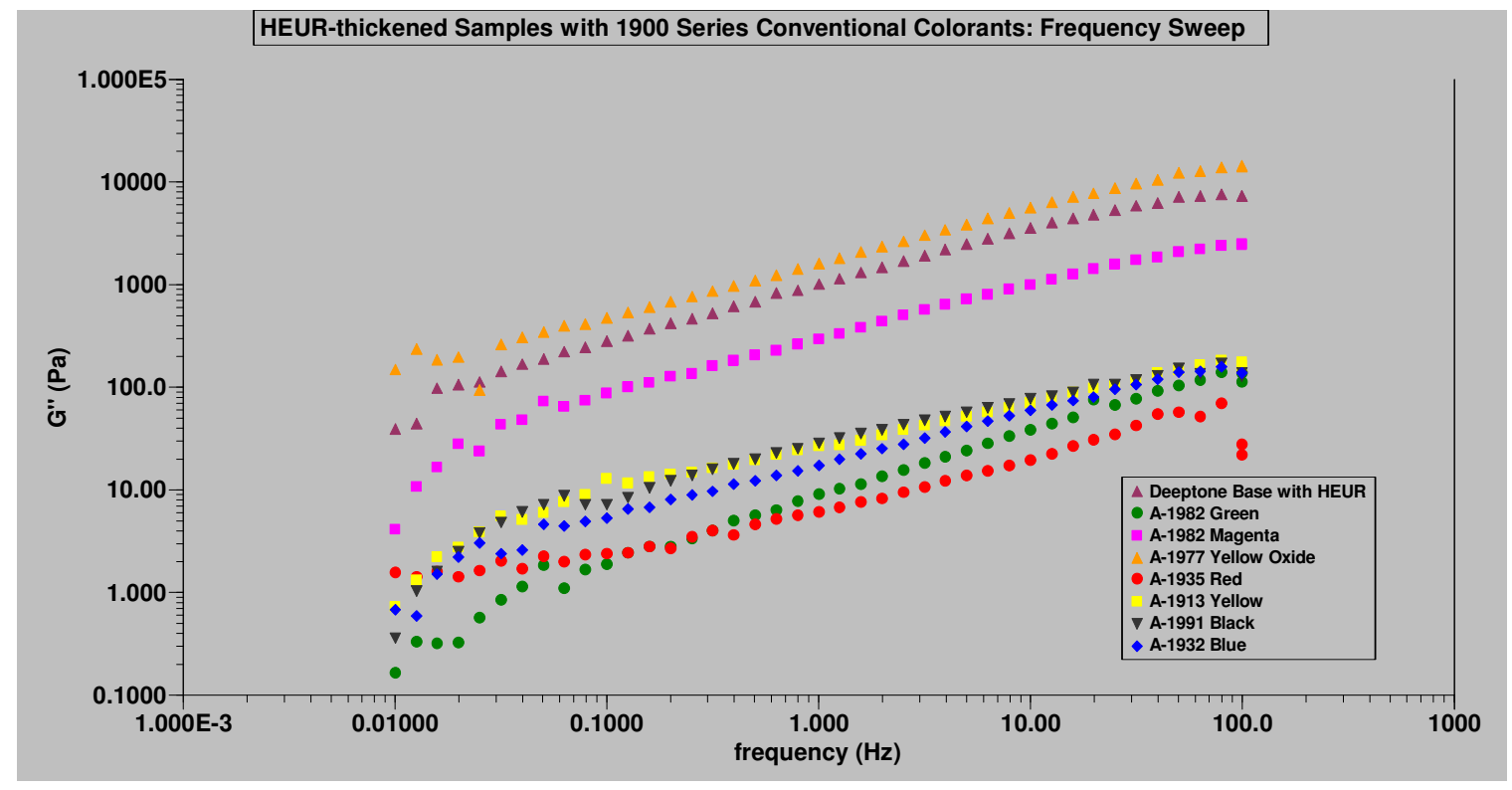

(d)

Figure 24. Frequency sweep of: HEC-thickened samples with 1900 series conventional colorants, elastic (a) and viscous (b) moduli, and HEUR-thickened samples with 1900 series conventional colorants, elastic (a) and viscous (b) moduli

3.2.9 Frequency Sweep of Samples by Thickener and Color

The HEC-thickened yellow, red, yellow oxide, and N-8821N green samples all had moduli clearly higher than the nontinted base. The increasing rate of deformation had a 
reinforcing effect in these samples. The moduli of all of the HEUR-thickened samples (except for the sample with yellow oxide conventional colorant) were lower than the base. Since the viscous moduli of each of the samples were higher than the elastic moduli in the strain sweep graphs, all of the HEUR-thickened samples are unstable toward flocculation and phase separation.

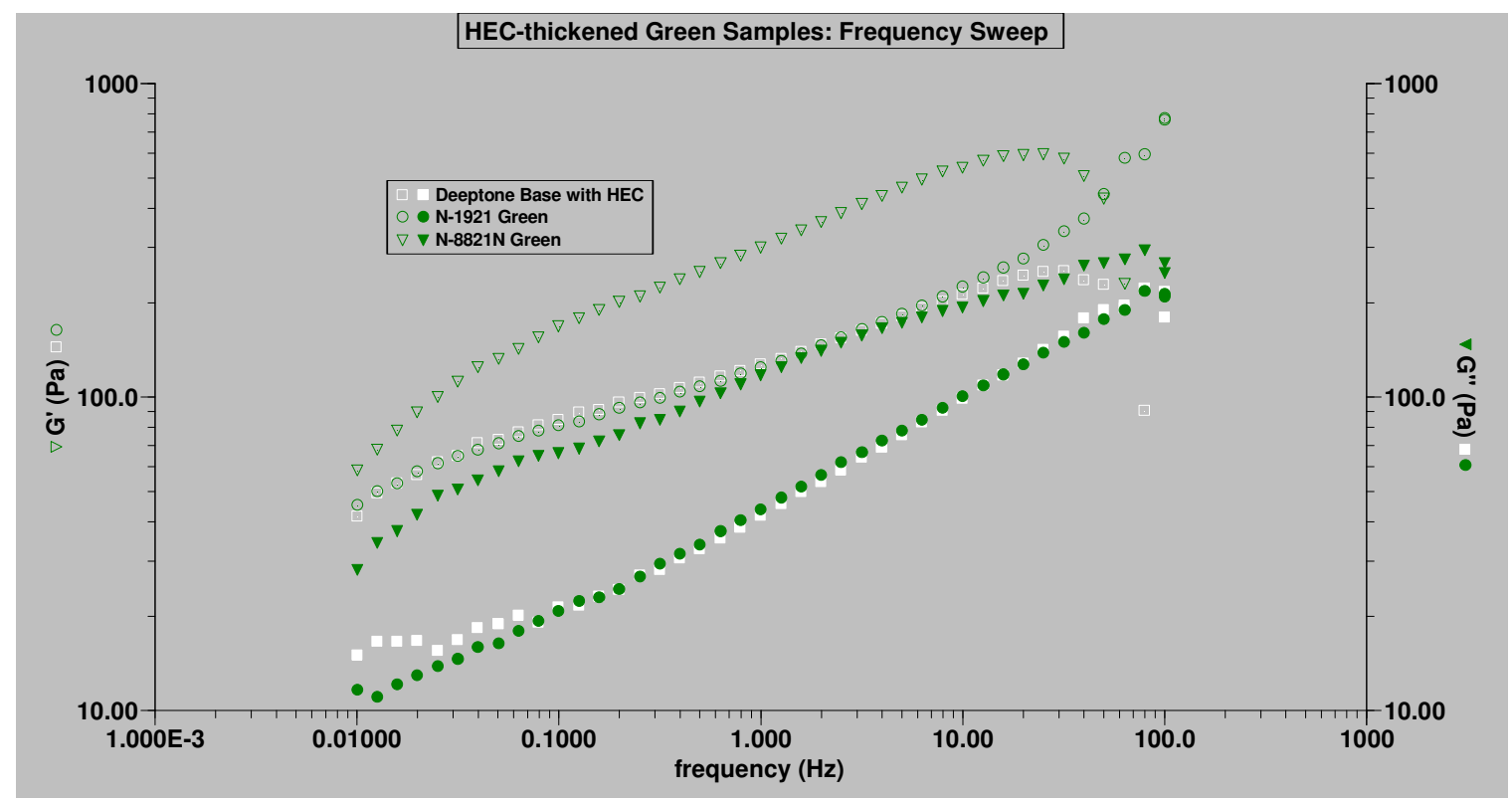

(a)

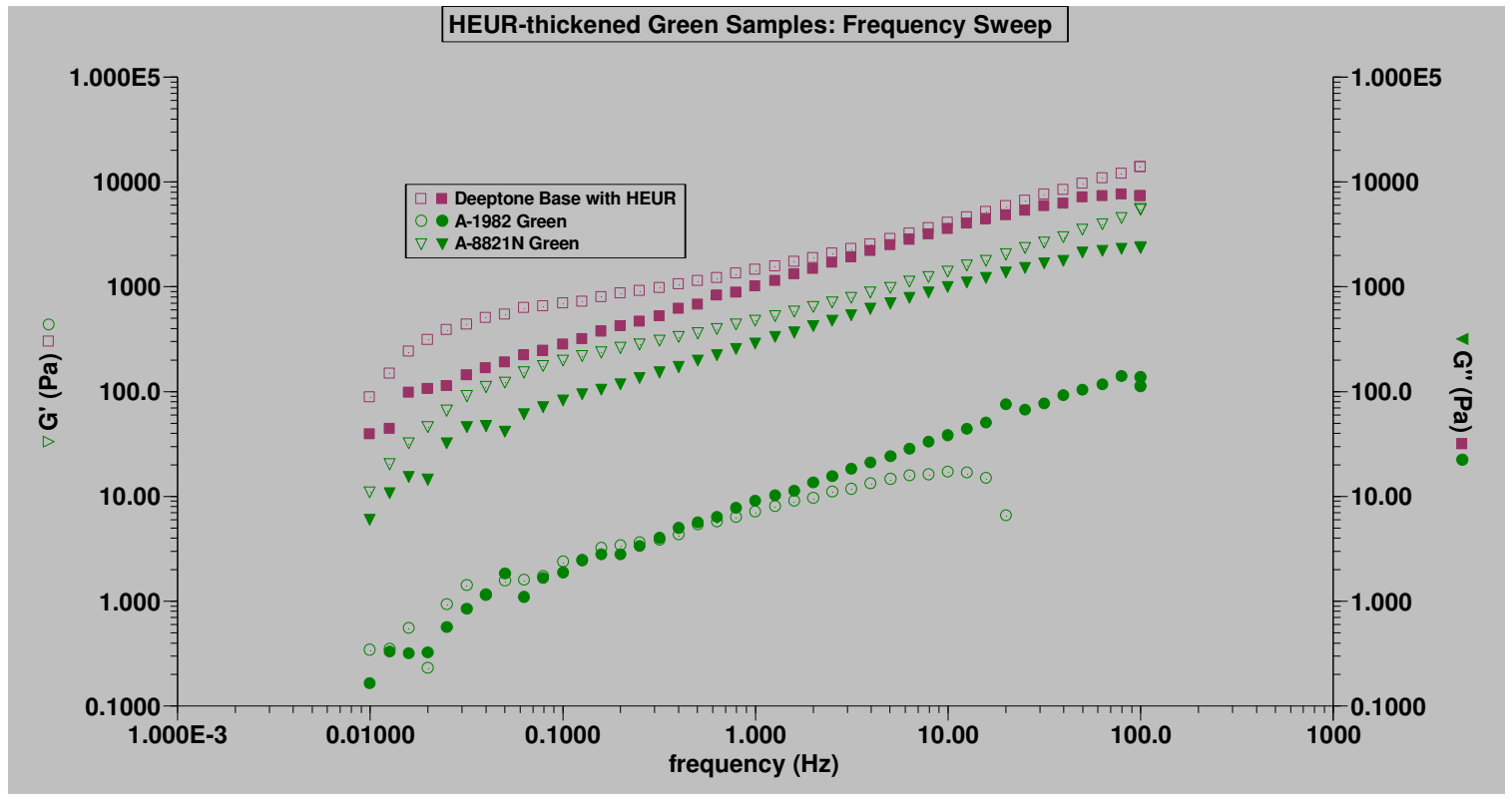

(b)

Figure 25. Frequency sweep of: (a) HEC-thickened samples with green colorant and (b) HEURthickened samples with green colorant 


\subsubsection{Gloss Analysis}

The gloss values of the deeptone bases very much reflect those of the pastel bases.

Almost every HEUR-thickened sample has a higher gloss than the sister HEC-thickened sample of the same colorant series. For most of the samples, the simple addition of the colorant reduced the gloss. This has nothing to do with the viscosity decrease, but the increase in pigmentation of the sample. The major factor controlling gloss is pigmentation; as coatings dry, the pigment particles can cause irregularities on the surface of the film. Therefore, when the pigment particles were added to the deeptone samples (in the form of colorants), the increase in pigment volume concentration (PVC) caused the reduction in gloss. Tint strength values were not able to be recorded for any of the deeptone samples. Coatings at 6, 15, and 21 millimeters thickness were made and the hiding power was not sufficient enough for tint strength measurement of any of these samples.

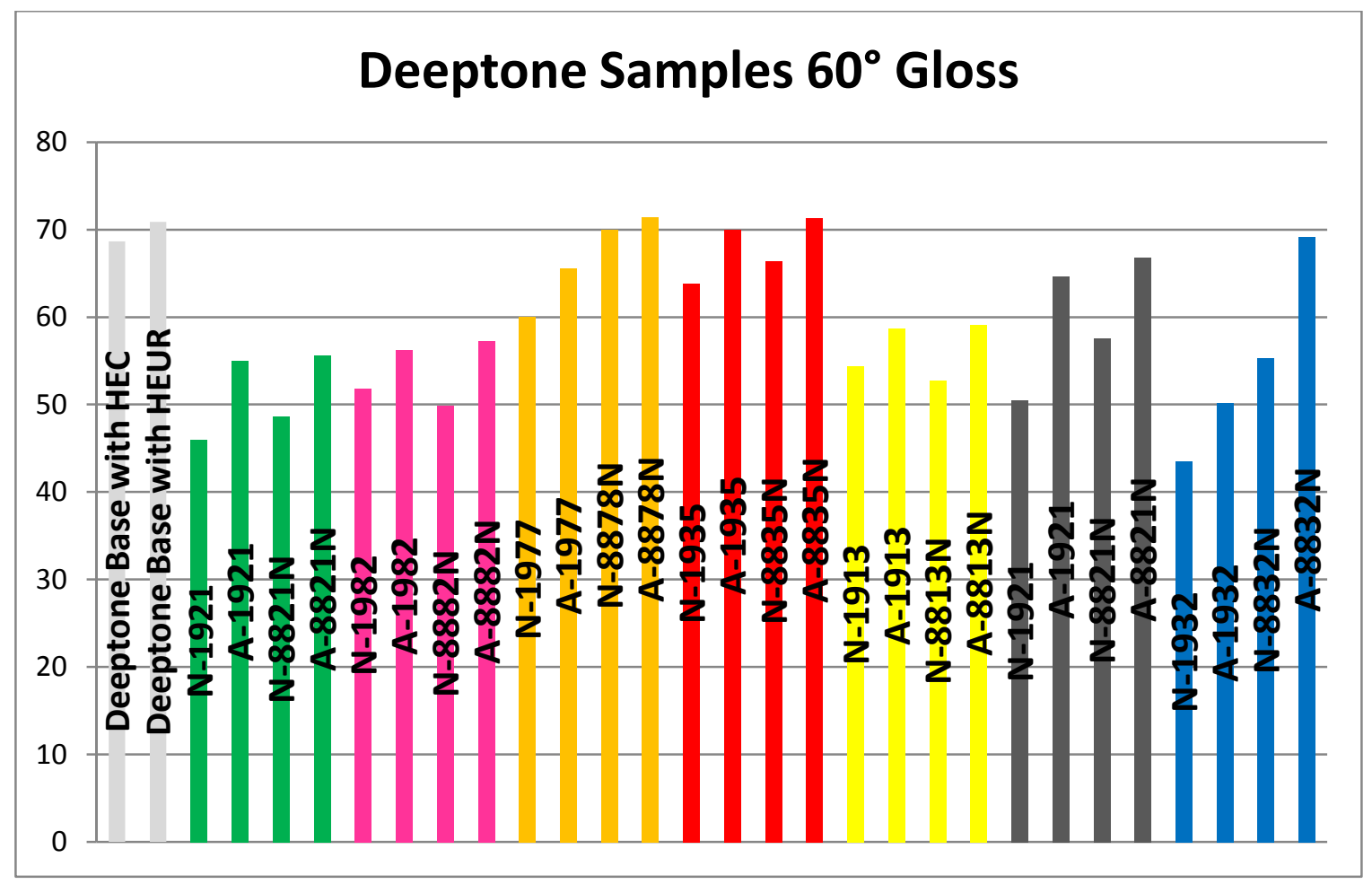

(a) 


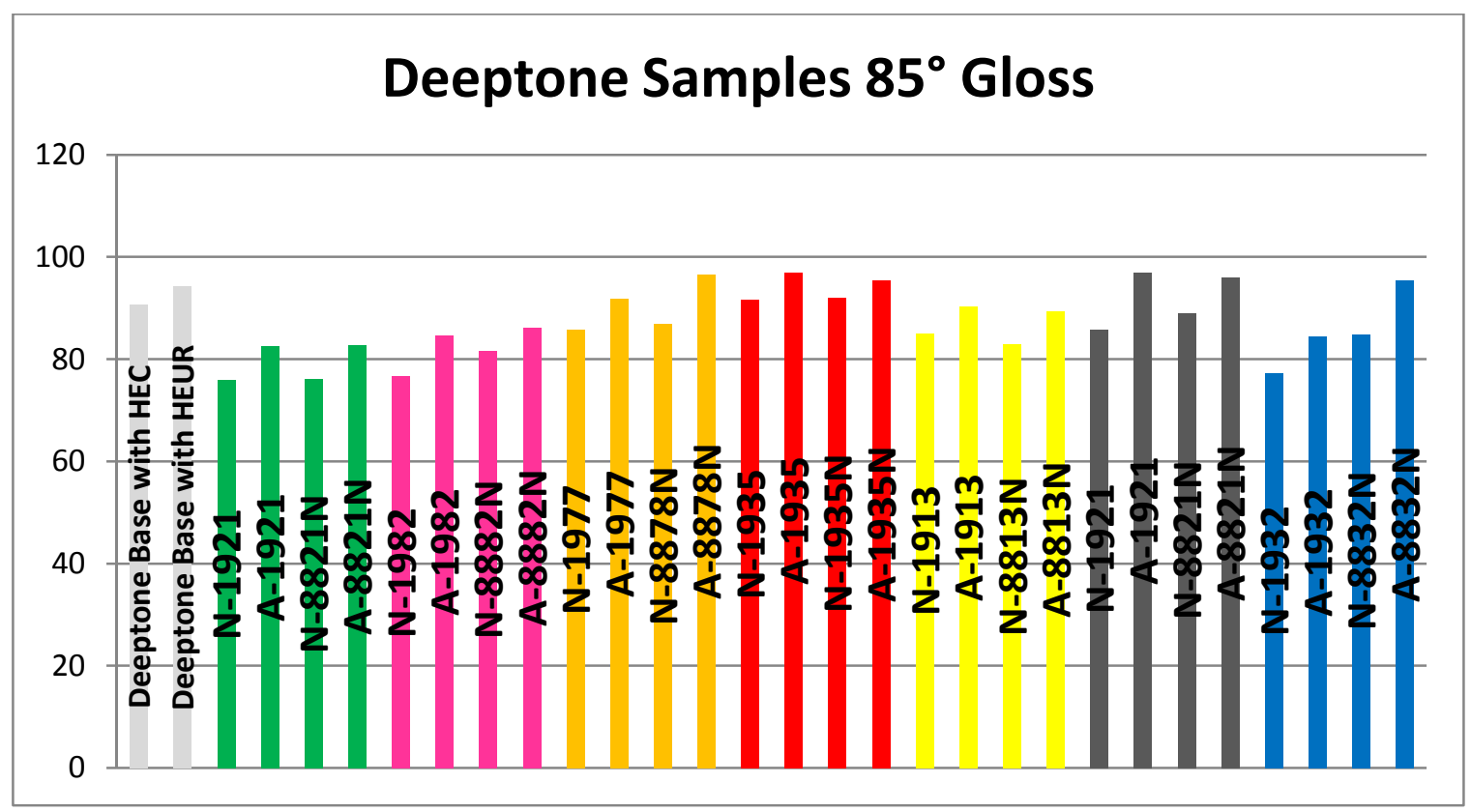

(b)

Figure 26. (a) $60^{\circ}$ gloss values of all deeptone samples and (b) $85^{\circ}$ gloss values of all deeptone samples

\subsection{Effect of Colorant Addition on Rheology of Paints with Different Coalescing Aids}

\subsubsection{Viscosity Profiles of Nontinted Bases}

The viscosity profiles of the nontinted bases are shown in Figure 27. All samples exhibited shearthinning behavior; with not much variation amongst them. The DOW formulation samples were just a little higher in Stormer viscosity than the Turk formulation samples. The Turk formulation is a little higher in density and solids content, which was unexpected based on the Stormer viscosity results (Table 5, 6). However, the difference between the Stormer viscosities of the paints was small enough to warrant this result. 


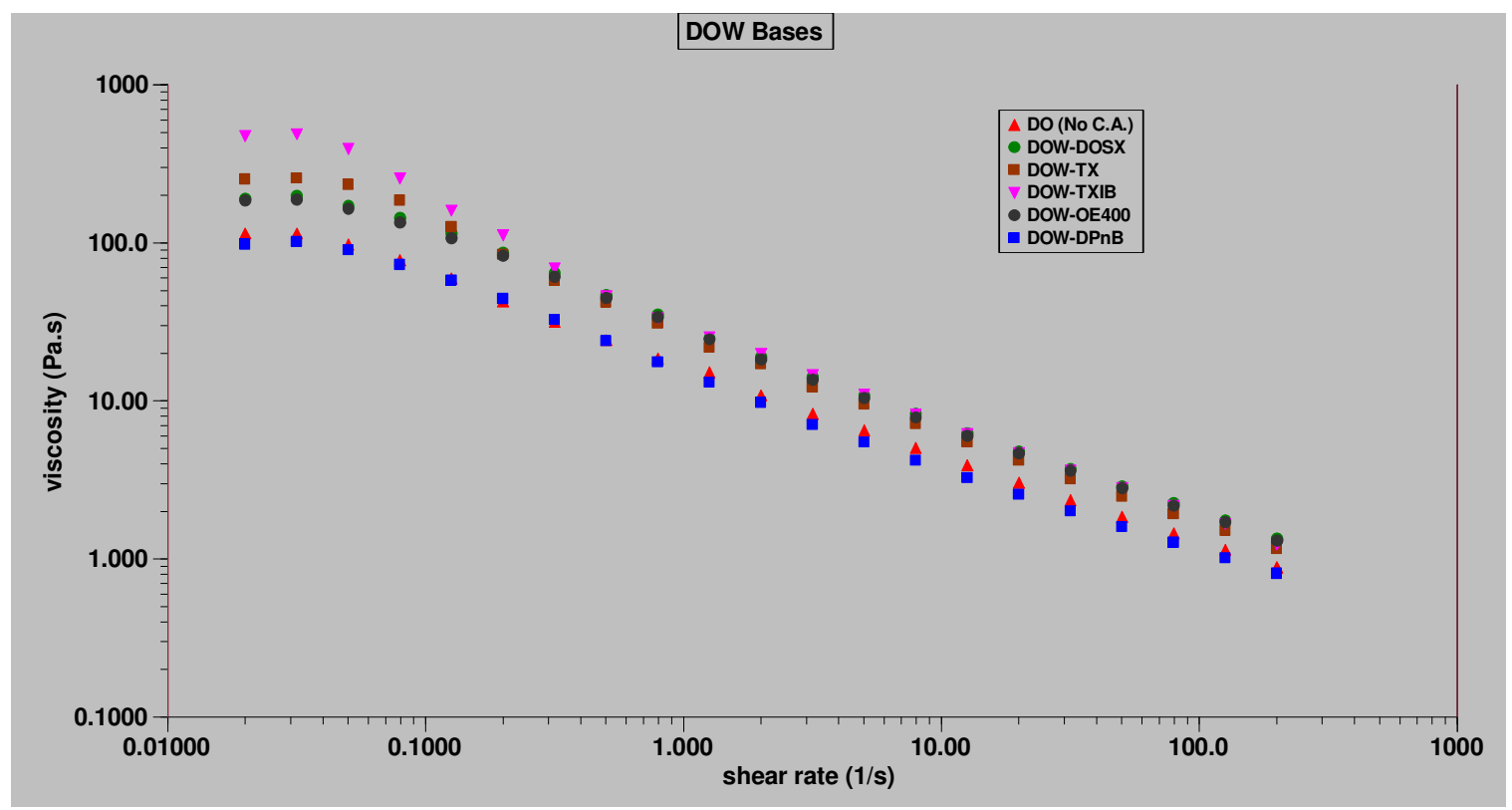

(a)

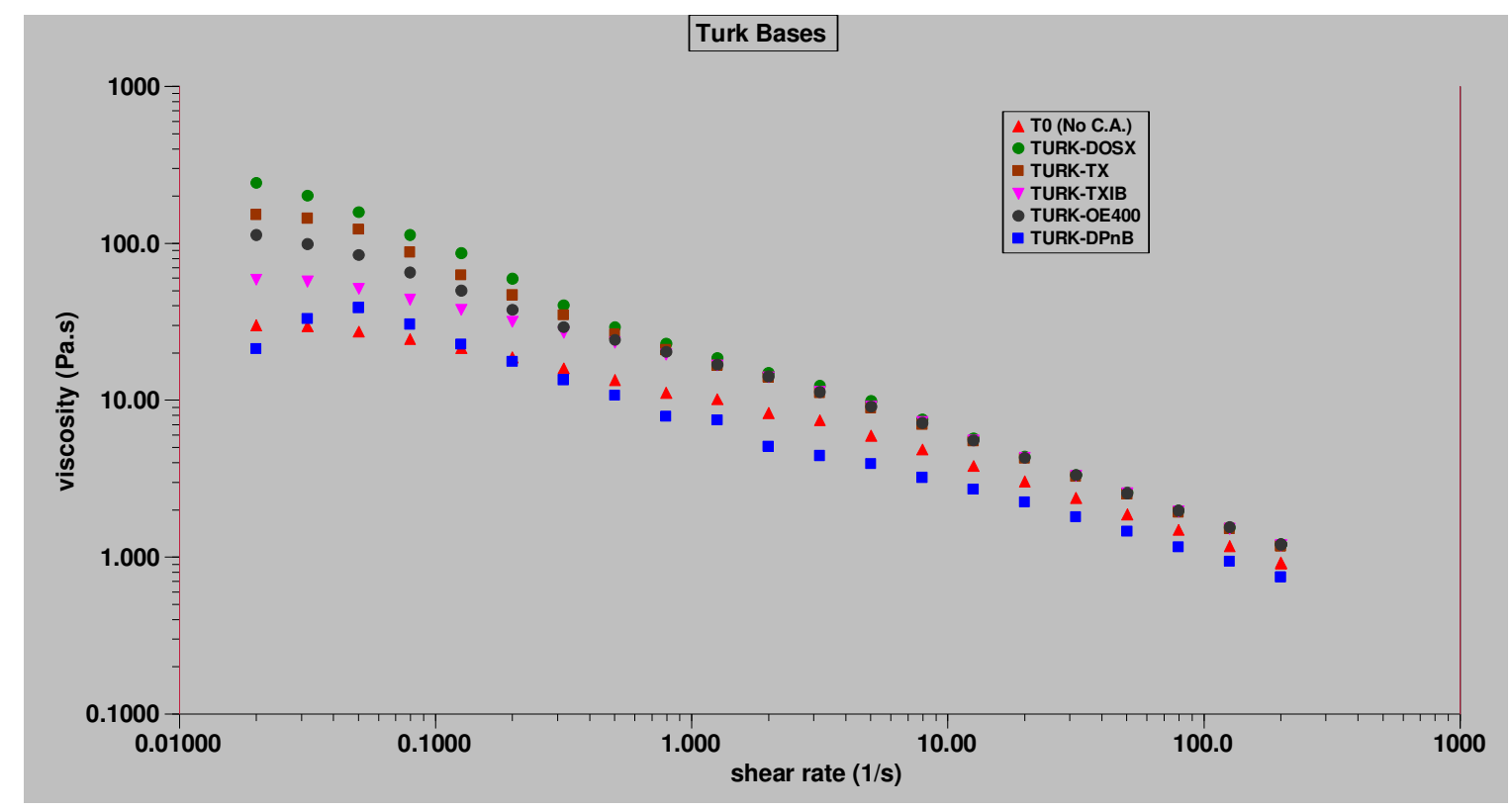

(b)

Figure 27. (a) DOW formulation nontinted bases, (b) Turk formulation nontinted bases 

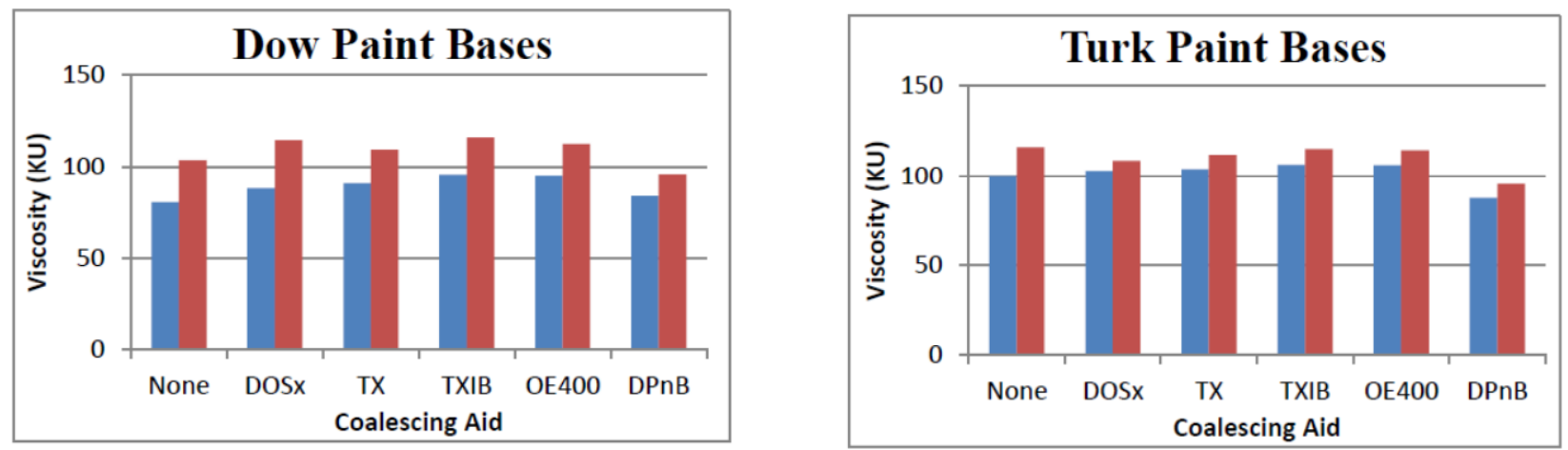

Figure 28. KU viscosities of Dow and Turk bases taken immediately after formulation ( $\square$ ), and

24 hours after formulation (

In both base formulations, the sample with dipropylene glycol n-butyl ether (DPnB) was

the lowest in viscosity. This was an interesting result considering that the only variation among

the bases was the type of coalescing aid used. It is possible that DPnB has a different rate of absorption into the latex particles, but this wouldn't cause a decrease of such magnitude seen here (Figure 6). Most likely, DPnB interferes with the associative thickener in such a way as to disrupt the viscosity-building hydrophobic interactions on a greater scale than the rest of the coalescing aids.

\subsubsection{Viscosity Profiles of Samples Arranged by Color}

When arranged by color, it is obvious that the rheological properties of the samples are similar as coalescing aid is varied, but not as the colorant is varied. Upon observation after 24 hours, the pigments of each of the Turk samples, and the Dow red samples showed a mild separation while the blue pigment of the Dow samples was severely flocculated. The yellow Dow samples were more or less completely homogeneous (Figure 29). 


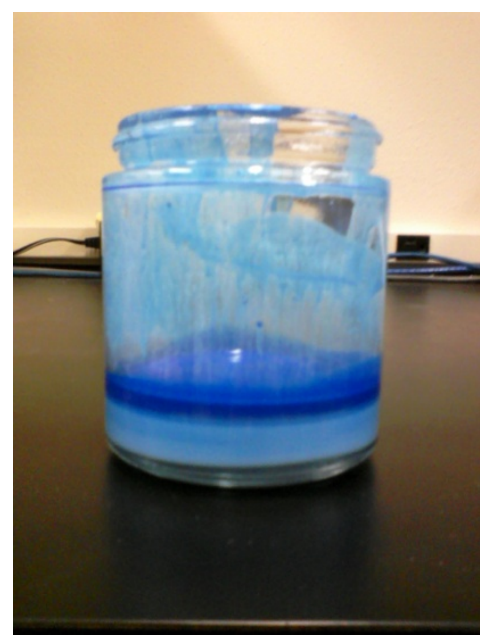

(a)

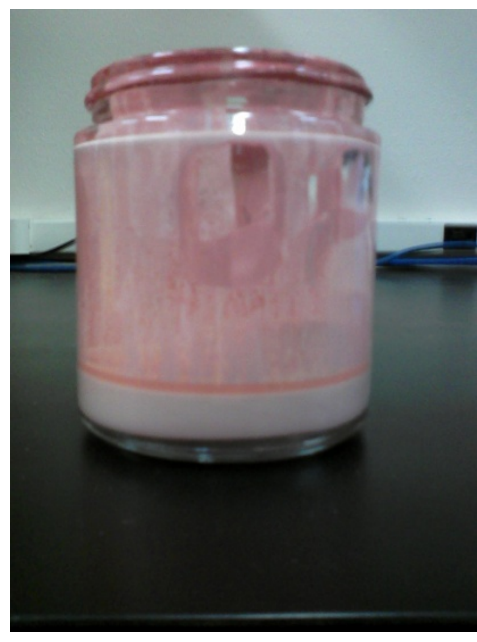

(b)

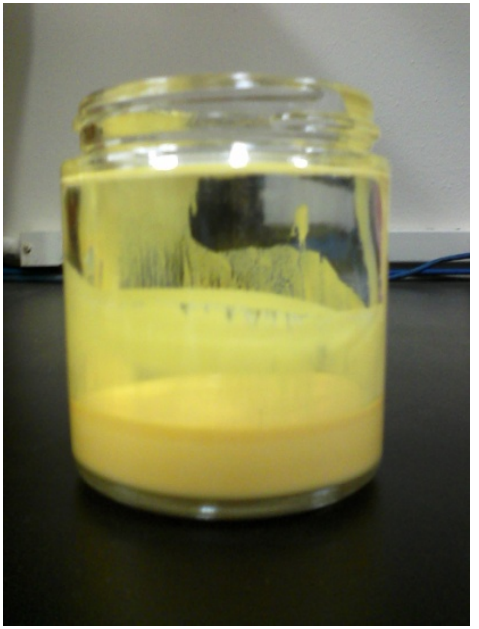

(c)

Figure 29. Tinted bases after storage; (a) DOW blue (DOSx), (b) DOW red (DOSx), (c) DOW yellow (DOSx)

Because each of the samples was mixed vigorously for 15 seconds with a spatula before testing, not much can be said to confirm the pigment flocculation from the rheology analysis. However, the blue samples of the Dow formulation had high low shear rate viscosities, and the readings were very erratic. This is typical rheological behavior of flocculated pigment dispersions. The blue pigment used in the CCA colorant was $\alpha$-phthalocyanine blue (PB15:2), which has been previously shown to be unstable with regards to flocculation during long periods of storage. The rest of the samples all exemplified typical shear-thinning behavior, with not much difference between those with different coalescing aids. Finally, the addition of a coalescing aid does not seem to have any effect on the rheological properties of the samples; the control sample curves (T0, D0) were more or less evenly matched with the rest (save for the DOW blue samples). 


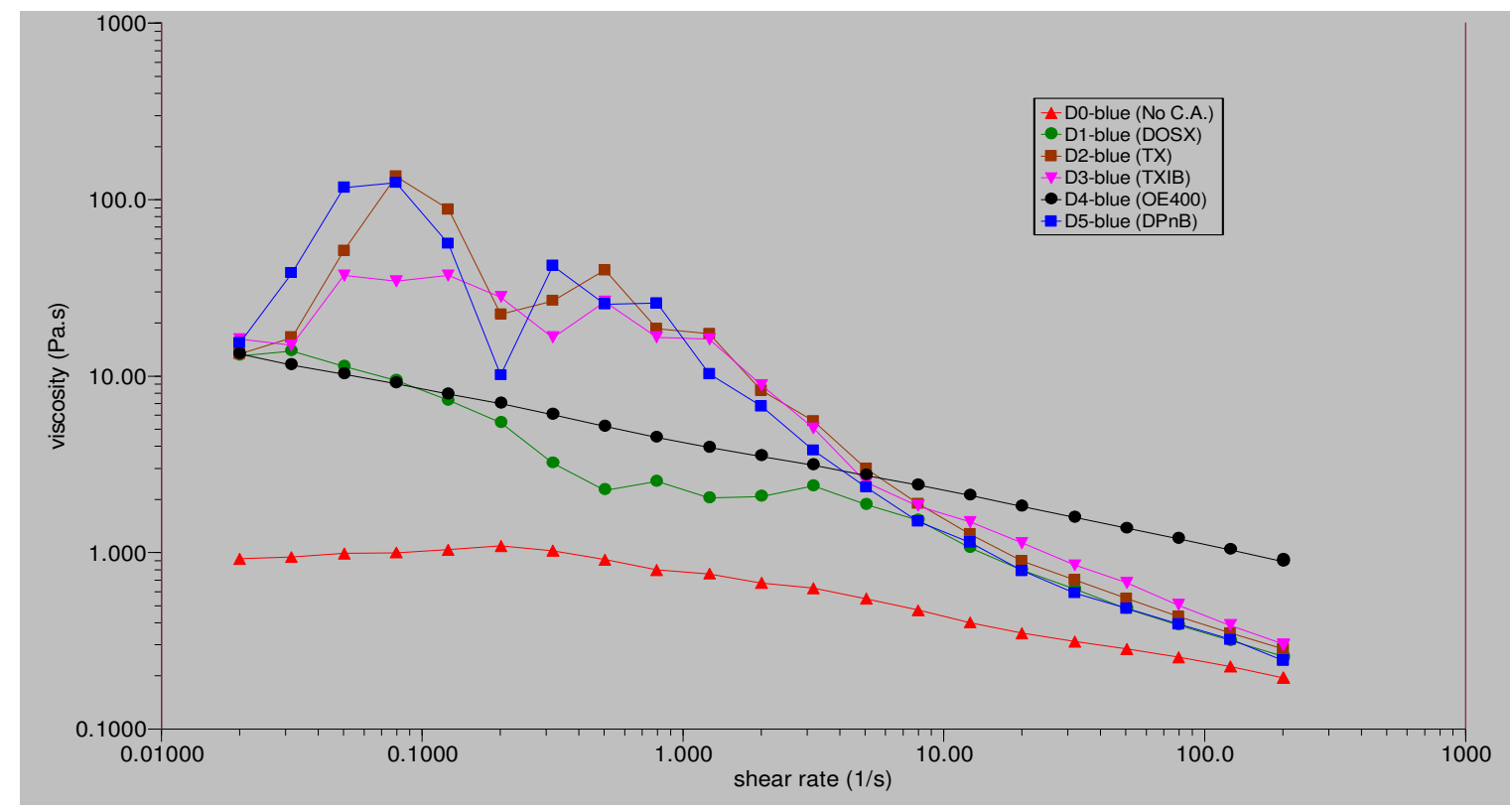

(a)

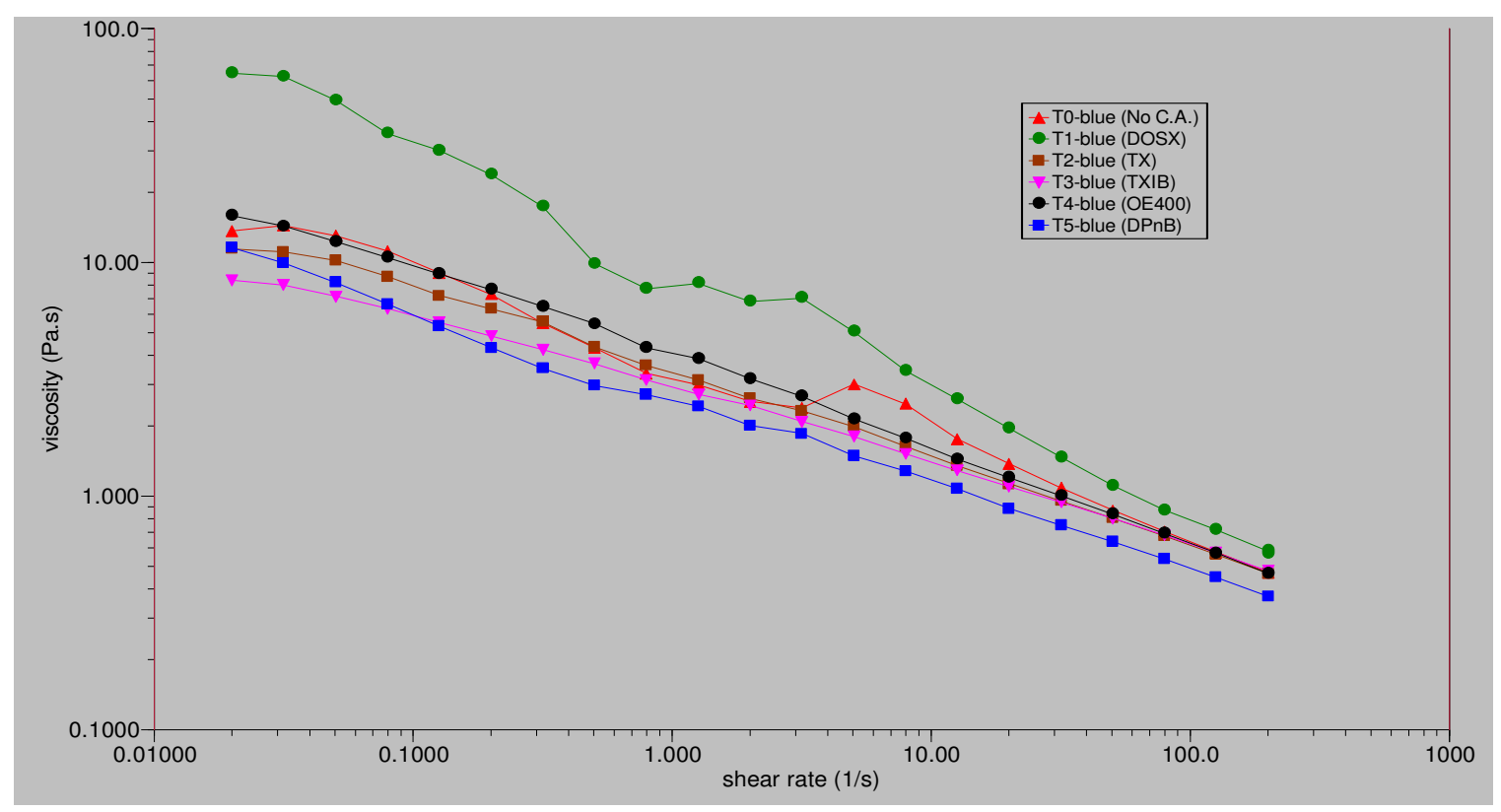

(b)

Figure 30. Viscosity profiles by color. (a) Dow formulation with blue colorant, (b) Turk formulation with blue colorant. 
3.3.3 Viscosity profiles of samples arranged by coalescing aid

The first thing that is noticed about each of these curves (Figure 31) is that the addition of colorant has a profound effect on the rheology of each sample; even those with no coalescing aid. All of the curves of the samples with colorant are shifted downward in regards to the samples with colorant. This direct evidence of viscosity loss may be ascribed to the network breakdown theory proposed by Landoll and Sau; there are certainly enough surfactants in the system to support this theory (Sau, 1989). The theory also makes intuitive sense, as the surfactants displace the hydrophobic interactions between the latex and associative thickeners, there would be a decrease in the effective volume fraction of the dispersed latex, reducing viscosity as a consequence. There have been previous studies that support this theory; Mahli, et al. tested the viscosities of solutions of HEUR-type thickeners with different concentrations of surfactants and found a Gaussian-type curve where there is an optimal concentration of surfactant that maximizes viscosity (Mahli, 2005). Because of the high concentration of surfactants in the tinted bases, it is believed that the samples tested here are on the far side of that curve. The high viscosity of the yellow Turk sample with no coalescing aid can be attributed to instrument error. The force necessary to produce oscillations at the low shear rate range is so small, erroneous values often occur as a result. 


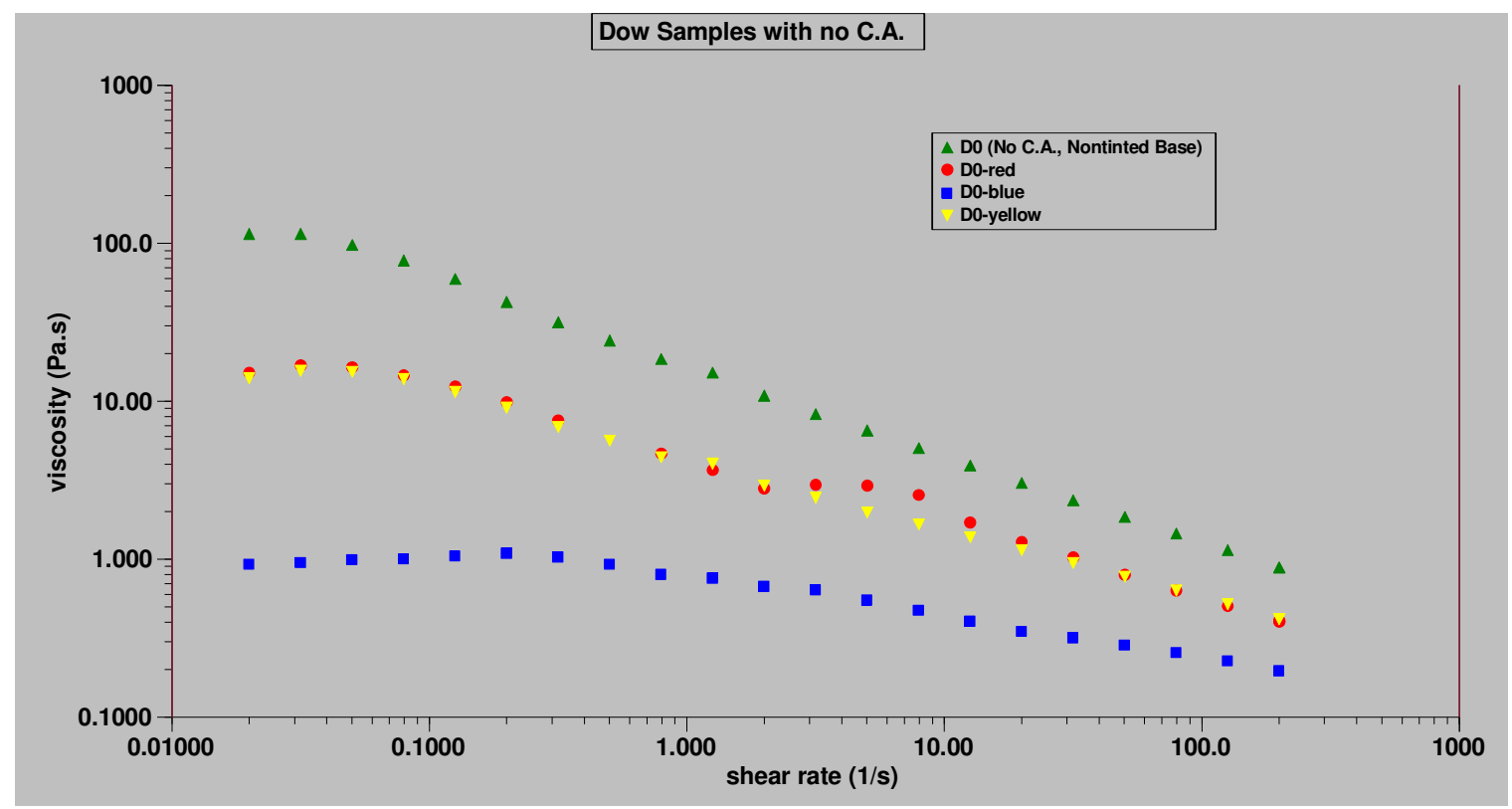

(a)

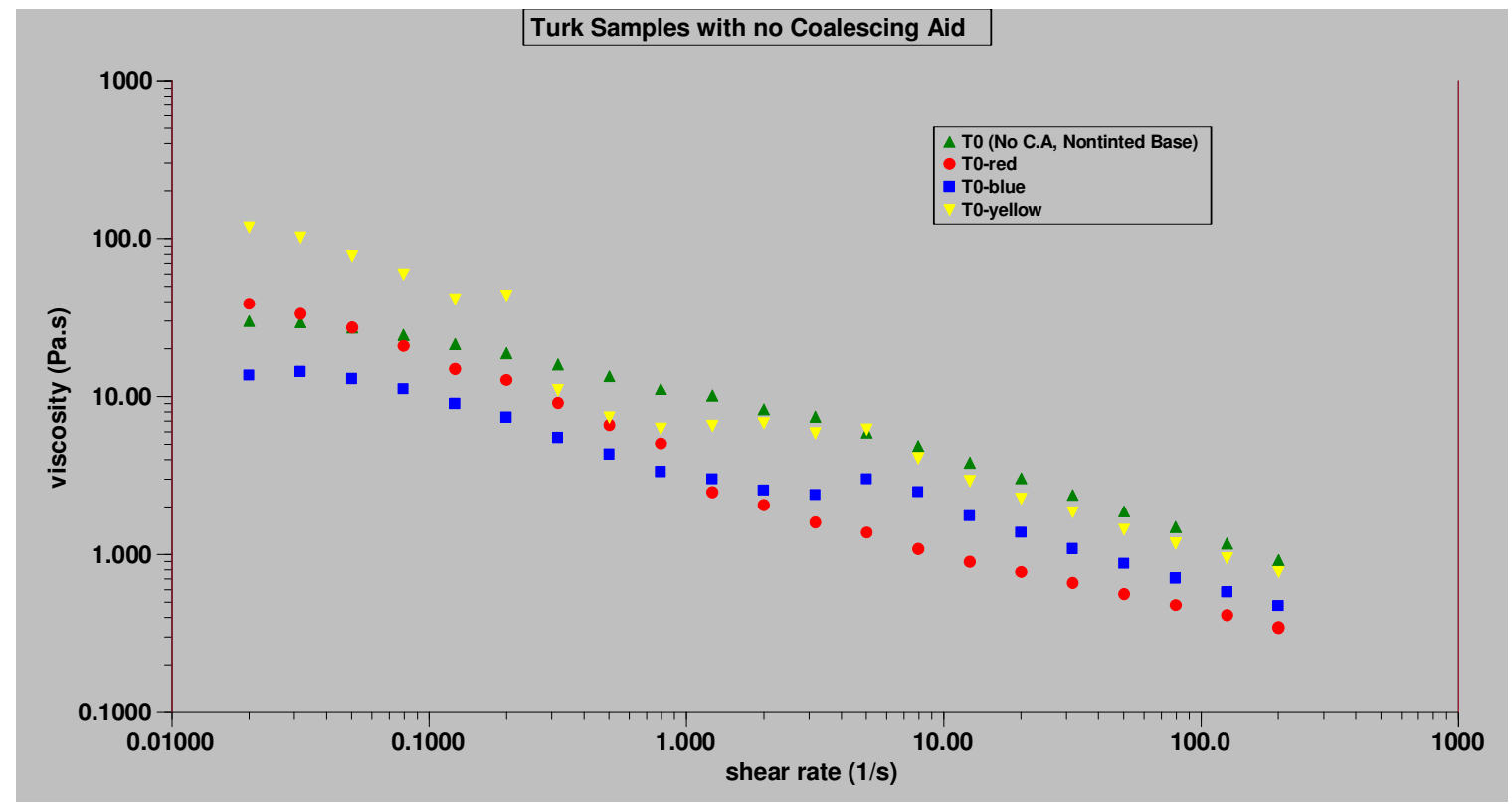

(b) 


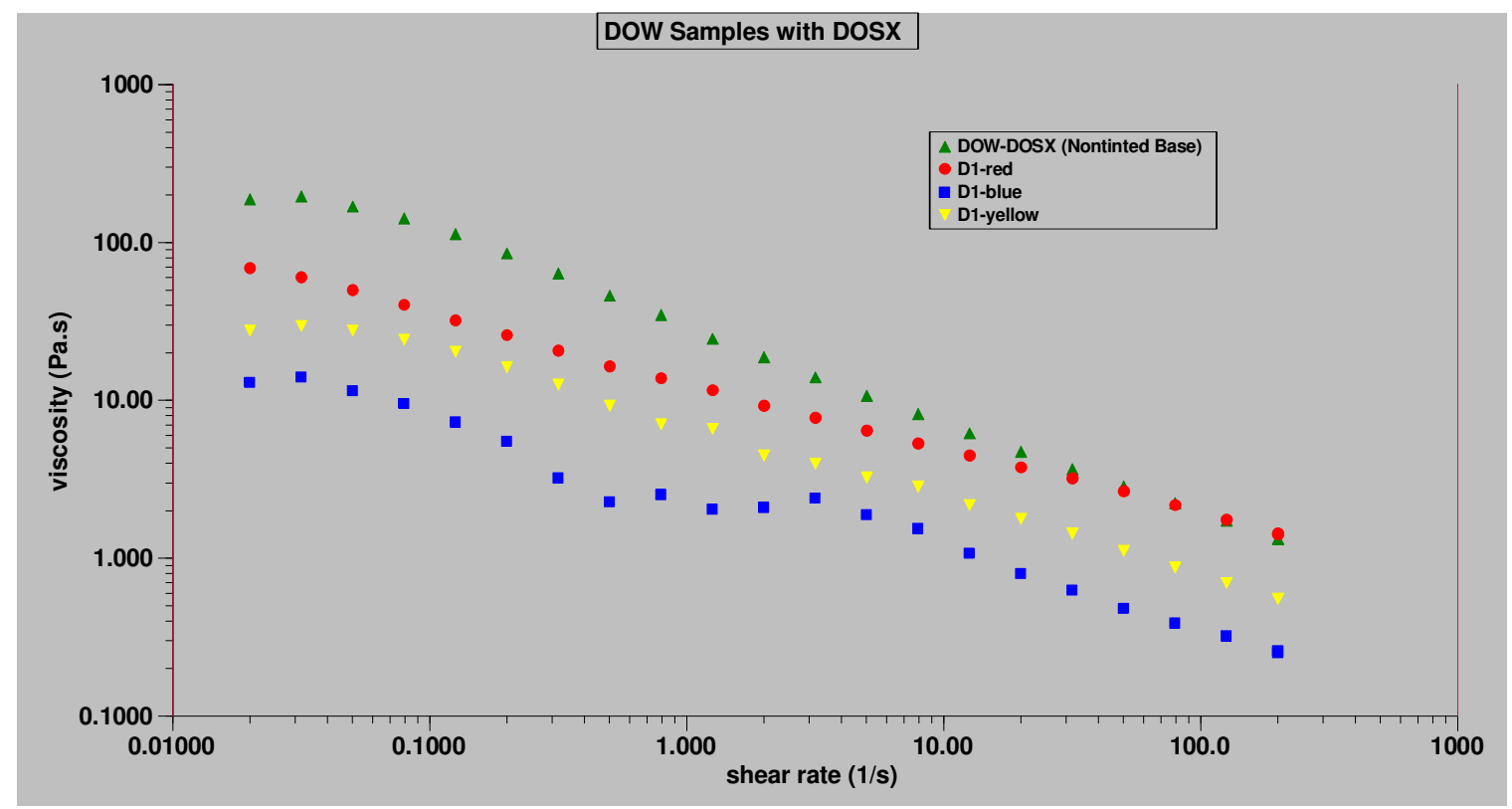

(c)

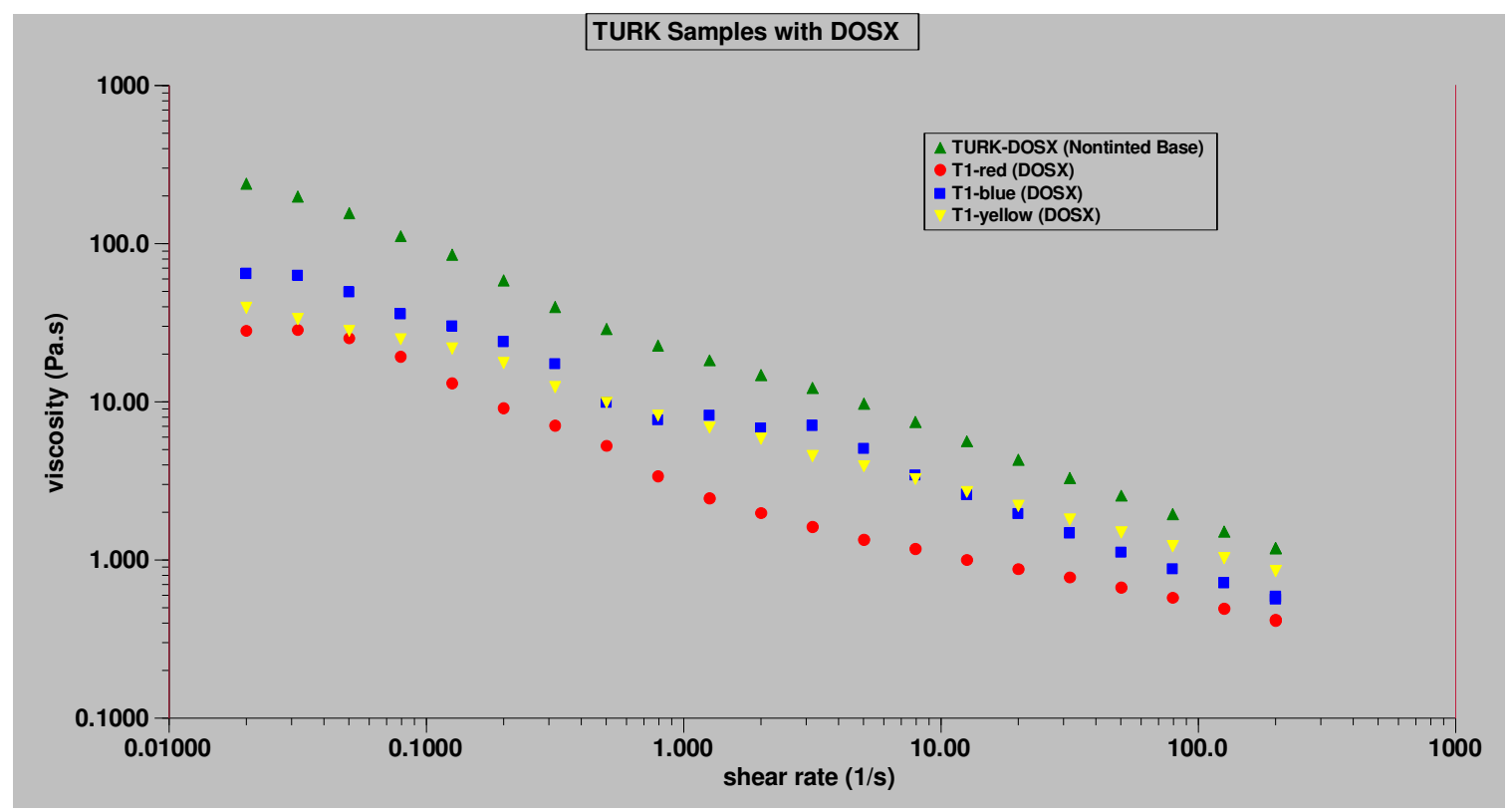

(d)

Figure 31. Viscosity profiles by coalescing aid. (a), (b) DOW and Turk formulations with no coalescing aid respectively, (c), (d) DOW and Turk formulations with DOSx respectively.

One important observation already noted above concerns the samples with blue colorant, which are consistently the lowest in viscosity of the group. This was attributed to the instability of the copper phthalocyanine blue pigment used in the blue colorants. Another peculiar result was 
that the DOW formulation red samples are all higher in viscosity than the DOW yellow samples, but the opposite is true for those of the Turk formulation. Without the knowledge of the composition of the colorants, it is difficult to pinpoint the exact cause for this phenomenon. It could be possibly because of the differences in the two different formulations; the Turk formulation uses a hydrophobic surfactant with an HLB of 1.6 (Dee Fo 3010A). Using a hydrophobic surfactant is a good thing; a hydrophobic surfactant will adsorb strongly onto a hydrophobic pigment. However, the short hydrophilic chain provides poor steric stabilization. In summary, the results have proven that the addition of colorant to a paint base results in a significant decrease in the viscosity of the system.

\section{Conclusion}

The goal of this study was to examine the phenomenon of viscosity decrease in tinted bases through rheological analysis. The results indicate that upon addition of colorant to a base, the viscosity is indeed reduced, but only in bases with HEUR associative thickeners. The HEUR associative thickeners build viscosity by forming a network system; the hydrophobic regions on the thickener polymers adsorb onto the surface of the latex particles and interact with the hydrophobic regions of other HEUR polymers. The surfactants compete with associative thickeners for adsorption sites on the latex polymer particles. As more and more surfactants are added, they eventually break down the network of hydrophobic interactions between the thickener and latex particles. Although this theory has not been verified, experimental evidence supports this mechanism.

It was shown that colorants with different pigments also have an effect on dispersion rheology. The size, shape (cylindrical, rod, etc.), concentration, and the chemical nature (organic vs. inorganic pigments, surface treatment, etc.) of different pigments can have a remarkable effect on dispersion rheology. Among all of the samples tested, the samples that were the most sensitive 
to colorant addition were those tinted with colorants with phthalocyanine blue, green, iron oxide red, and carbon black pigments. Across the board, the samples that were the most resistant to changes in viscosity upon colorant addition were those tinted with colorants containing yellow oxide pigments.

Finally, the pigment volume concentration plays a big role in the extent to which colorant addition affects the rheology of tint bases. In this study, the deeptone bases had much lower PVC's than the pastel bases, but higher latex polymer concentrations. The addition of the associative thickener had much more of an impact on the change in viscosity upon colorant addition to the deeptone base as opposed to the pastel base. This is because of the higher concentration of latex particles available for network formation. The larger concentration of latex particles in the deeptone base vs. the pastel base provided the surfactants from the colorant with more adsorption sites. Therefore, less thickener molecules were displaced from the latex polymer surfaces upon colorant addition in the deeptone base compared to the pastel base, and the viscosities of the deeptone formulations were not lowered as much as the viscosities of the pastel formulations. Future work on this project would involve colorants of a known formulation so that the actual concentration and species of surfactants in the system could be varied. Specific rheological behavior could be attributed directly to the concentration or species of surfactant. There are many other variables (latex particle size, latex concentration, latex species, and dispersant for example) that could also be included in the study. 


\section{References}

Annable, T.; Buscall, R.; Ettelaie, R.; Whittlestone, D. The Rheology of Solutions of Associating Polymers: Comparison of Experimental Behavior with Transient Network Theory. $J$. Rheol. 1992, 37, 695-725.

Coleman, M.M.; Painter, P.C. Fundamentals of Polymer Science. 2nd Ed.; CRC Press LLC. Boca Raton, Flordia. ㄷ 1997.

Dziwok, K. Associative Thickeners for Viscosity Retention Upon Thickening. Paint and Coatings Industry. [Online]. http://www.pcimag.com/articles/associative-thickeners-forviscosity-retention-upon-tinting

English, R.J.; Laurer, J.H.; Spontak, R.J.; Khan, S.A. Hydrophobically Modified Associative Polymer Solutions: Rheology and Microstructure in the Presence of Nonionic Surfactants. Ind. Eng. Chem. Res. 2002, 41, 6425-6435.

Erbil, H.Y. Interfacial Interactions of Liquids, Somasundaran, P. et al. Colloid Systems and Interfaces-Stability of Dispersions through Polymer and Surfactant Adsorption. In Handbook of Surface and Colloid Chemistry; Birdi, K.S., Ed.; CRC Press LLC: Florida, 1997; Ch. 2, 14.

Fernando, R.H. Rheology Modifiers. Chemistry 550 Presentation. Cal Poly San Luis Obispo Polymers and Coatings. Spring 2011

Fernando, R.; Johnson, L.; Manion S.; Shear-Thickening in Aqueous Surfactant-Associative Thickener Mixtures. J. Coat. Technol. Res. 2011, 8 (3), 299-309.

Fernando, R.H. Rheological performance of Associative Thickeners and Cellulose Ethers in Architectural Coatings. Presented at Asia Pacific Coatings Conference, Kuala Lumpur, Malaysia (2003).

Ferry, J.D. Viscoelastic Properties of Polymers, 3rd Ed.; John Wiley \& Sons, Inc., 1980.

Glass, J.E. Advances in Colloid and Interface Science, 1999, 99, 123-148.

Glass, J.E. Influence of Water-Soluble Polymers on Rheology of Pigmented Latex Coatings. Water-Soluble Polymers, 1986, 391-416.

Hester, R.D.; Squire Jr.; D.R. Rheology of Waterborne Coatings. J. Coat. Tech. 1997, 69 (864), 109-114.

Jones, F.; Pappas, S.; Wicks, D.; Wicks Jr., Z. Organic Coatings: Science and Technology. $3^{\text {rd }}$ ed.; Wiley-Interscience. John Wiley \& Sons, Inc. Hoboken, New Jersey. @ 2007.

Ma, Z.; Chen, M.; Glass, J.E. Adsorption of Nonionic Surfactants and Model HEUR Associative Thickeners on Oligomeric Acid-Stabilized Poly(Methyl Methacrylate) Latices. Colloids and Surfaces A. 1996, 112, 163-184. 
Mahli, D. M.; Wegner, J. M.; Glass, J. E.; Dakota, N.; Phillips, D. G.; \& Corp, D. Waterborne Latex Coatings of Color: II. Surfactant Influences on Color Development And Viscosity. J. Coat. Technol. Res. 2005, 2 (8), 635-647.

Mahli, D.M.; Steffenhagen, M.J.; Xing, L.; Glass, J.E. Surfactant Behavior and its Influence on the Behavior of Associative Thickeners, Solutions, Thickened Latex Dispersions, and Waterborne Latex Coatings. J. Coat. Tech. 2003, 75, 39-51.

Malvern. Derjaguin, Landau, Verwey, and Overbeek (DLVO) Theory. http://www.malvern.com/labeng/industry/colloids/dlvo_theory.htm (accessed Nov 11, 2012).

Mezger, T.G. The Rheology Handbook, $2^{\text {nd }}$ Ed.; Vincentz Network, Hannover, Germany, 2006.

Mysels, K.J. Introduction to Colloid Chemistry, Interscience, New York, 1959.

Nisogi, H.; Bousfield, D.W.; Lepoutre, P.F. Influence of Coating Rheology on Final Coating Properties. Tappi Journal. 83 (2), 100-106, (2000).

Nisogi, H.; Bousfield, D.W.; Lepoutre, P.F. The Influence of Dynamic Rheological Behavior on Final Coating Properties: Part 1-Relationships Between Suspension Rheology at Equilibrium State and Dry Coating Bulk. Tappi Journal. 1999 Coating Fundamentals Symposium Proceedings, (1999).

Palierne, J. Rheothermodyamics of Transient Networks. 2000, http://arxiv.org/pdf/condmat/0007368.pdf

Paranthaman, Ashokkumar. Why Grind It? Paint and Coatings Industry. [Online]. http://www.pcimag.com/articles/why-grind-it-1

Parfitt, G.D. Ed. Dispersion of Powders in Liquids. 2nd ed.; Applied Science Publishers Ltd: London 1973.

Pascal, R.H.; Reig, F.L. Off. Dig. Federation Soc. Paint Technol., 1964, 36, 839.

Patton, T.C. Ed. The Pigment Handbook. Volume 1; Wiley Interscience: New York, 1973.

Quemada, D. Rheological Modeling of Complex Fluids. 1. The Concept of Effective Volume Fraction Revisited. Eur. Phys. J. 1998, 1, 119-127.

Sau, A., Landoll, L.M. Polymers in Aqueous Media, Performance Through Association, Advances in Chemistry 223, Chap. 18, Glass, J.E. (Ed.), American Chemical Society, Washington, D.C., 1989.

Saucy, D. Avoiding Viscosity Loss on Tinting. Paint \& Coatings Industry, August 2008, pp 3438. 
Schmitdz, J.; Frommelius, H.; Pegelow, U.; Schulte, H.G.; Höfer, R. A New Concept of Dispersing Agents for Aqueous Coatings. Progress in Organic Coatings. 1999, 35, 191196.

Shay, G.D. Thickeners and Rheology Modifiers In Paint and Coating Testing Manual; Koleske, J.V., Ed.; ASTM: Michigan, 1995; $14^{\text {th }}$ Edition; p 271-273.

Stollmaier, F.; Flink, P.; Forsberg, S.; Gradin, P.A. Influence of Latex Modifications on Viscoelastic Properties of Coating Colours. Tappi Journal. 1998 Coating Conference Proceedings, (1998).

Svanholm, T., Kronberg, B., Molennar, F. Progress in Organic Coatings, 1997, 30, 167-171.

TA Instruments. Reference Manual: Rheology of Fluids Using the AR2000ex Advanced Rheometer System. http://www.docstoc.com/docs/49619422/Reference-ManualRheology-of-Fluids-Using-the-AR2000ex-Advanced-Rheometer-System-Section-1Theory-of-Rheology-Part-1-Introduction-and-General-Theory-of-Rheology-Part-2Viscometric-Theo

Wicks Jr. Z.; Jones, F.; Pappas, S.; Wicks, D. “Organic Coatings: Science and Technology, Third Edition.” Wiley-Interscience. (C) 2007 by John Wiley \& Sons, Inc.

Zollinger, H. Color Chemistry, $2^{\text {nd }}$ Ed.; VCH, New York, 1991. 


\section{Appendix 1}

Viscosity Profiles of Pastel Samples Arranged by Thickener and Colorant Series
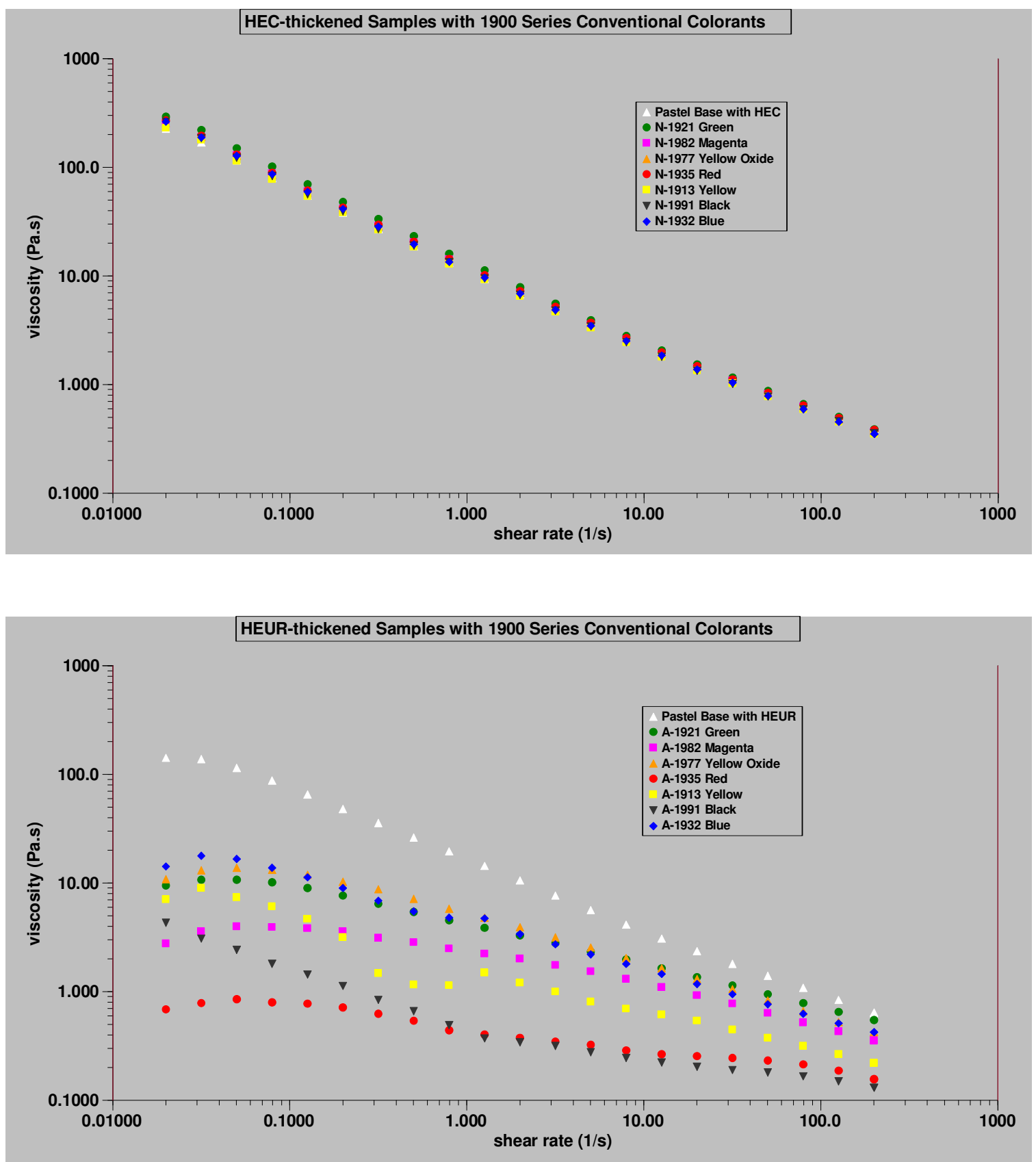


\section{Appendix 2}

\section{Viscosity Profiles of Pastel Samples Arranged by Color}
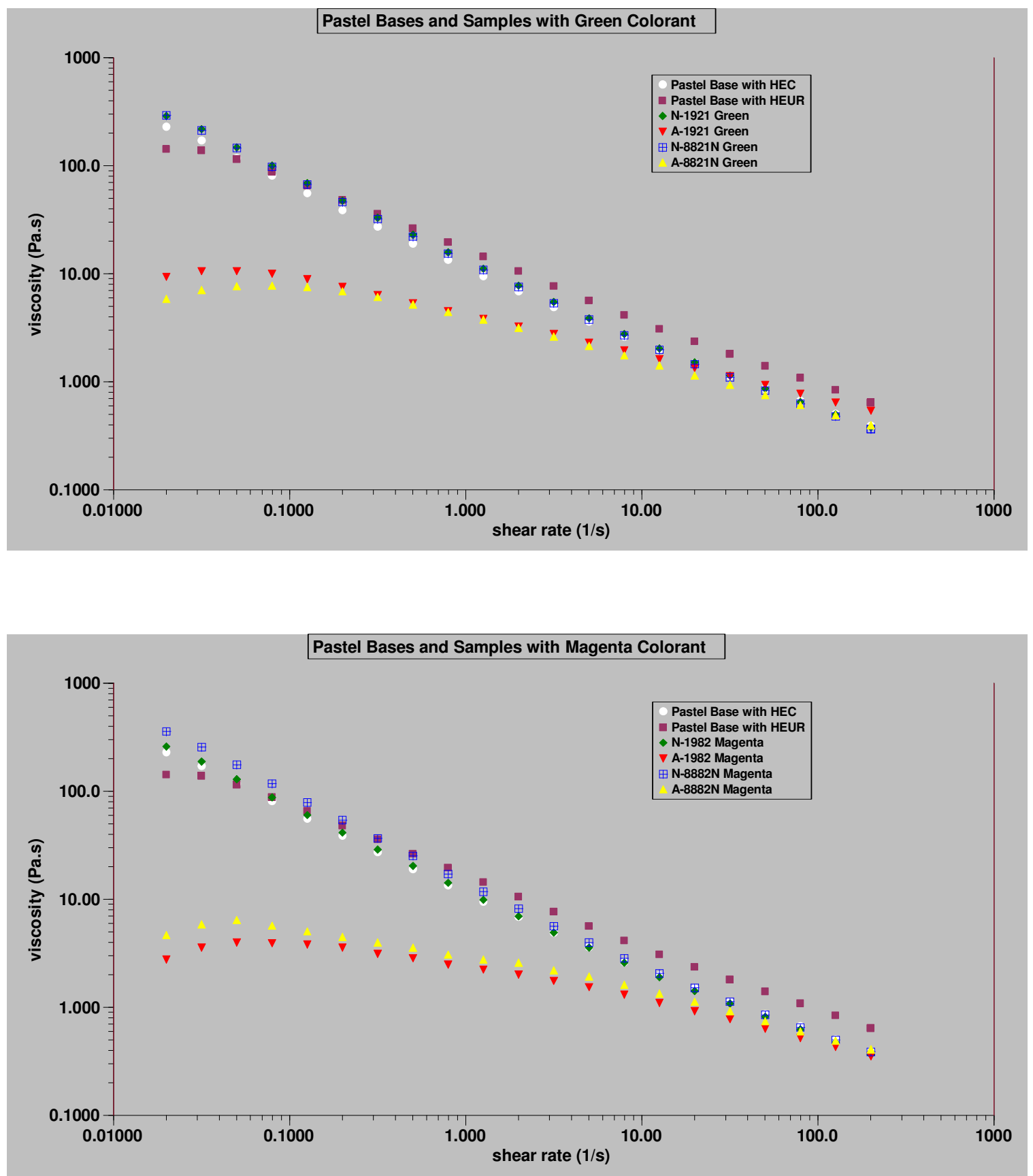
Pastel Bases and Samples with Red Colorant

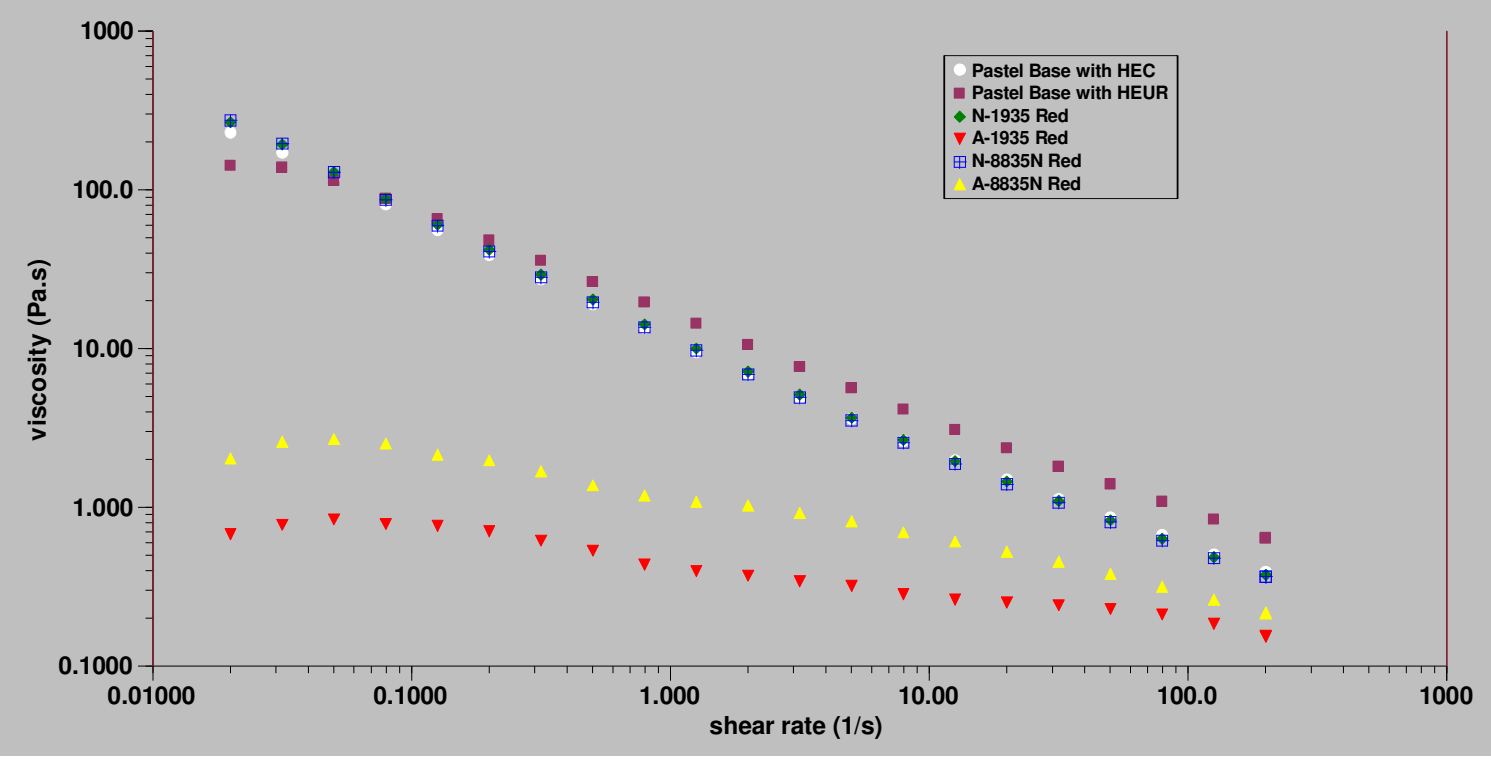

Pastel Bases and Samples with Yellow Colorant

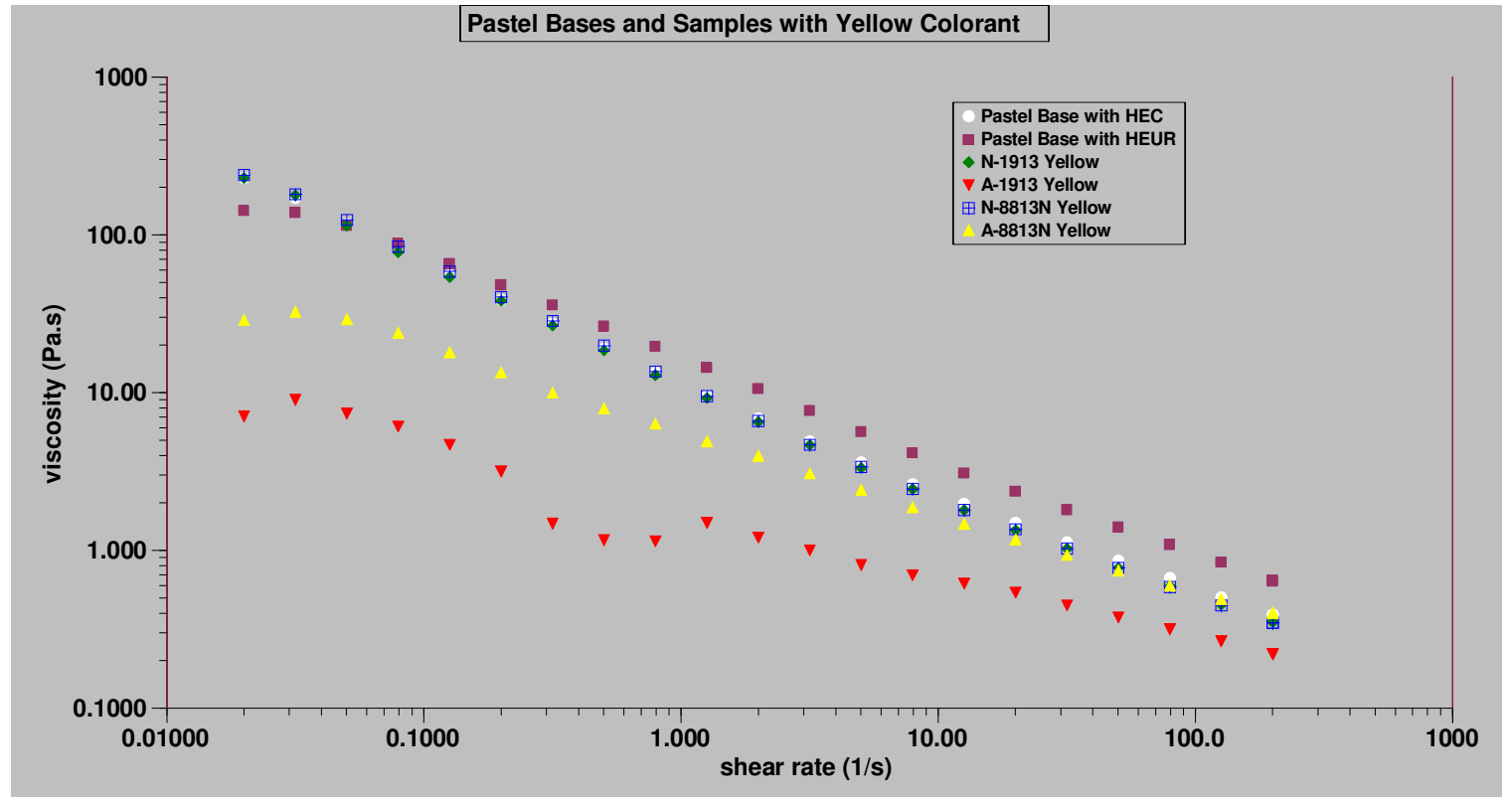




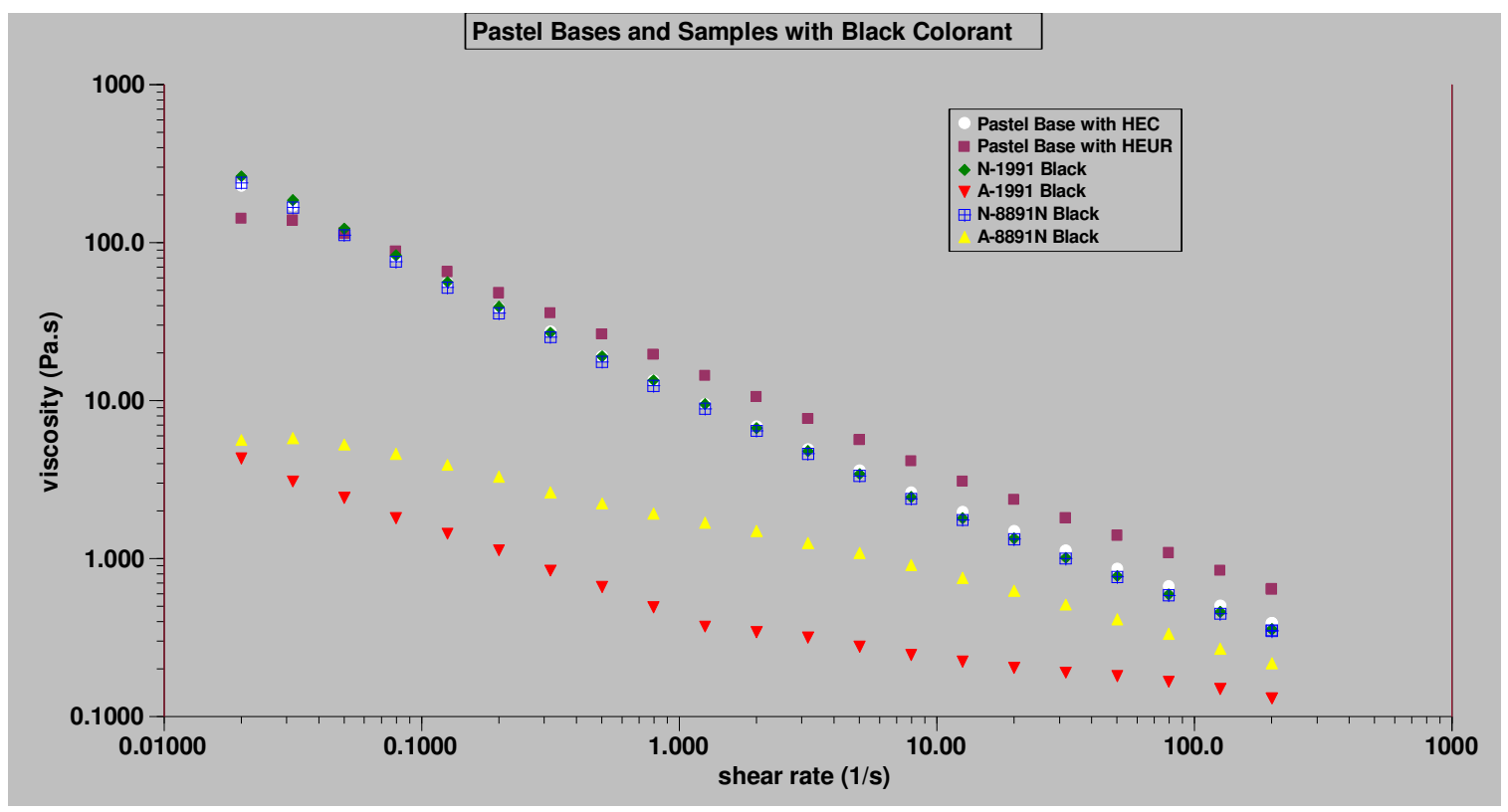




\section{Appendix 3}

\section{Strain Sweep of Pastel Samples Arranged by Thickener and Colorant Series}
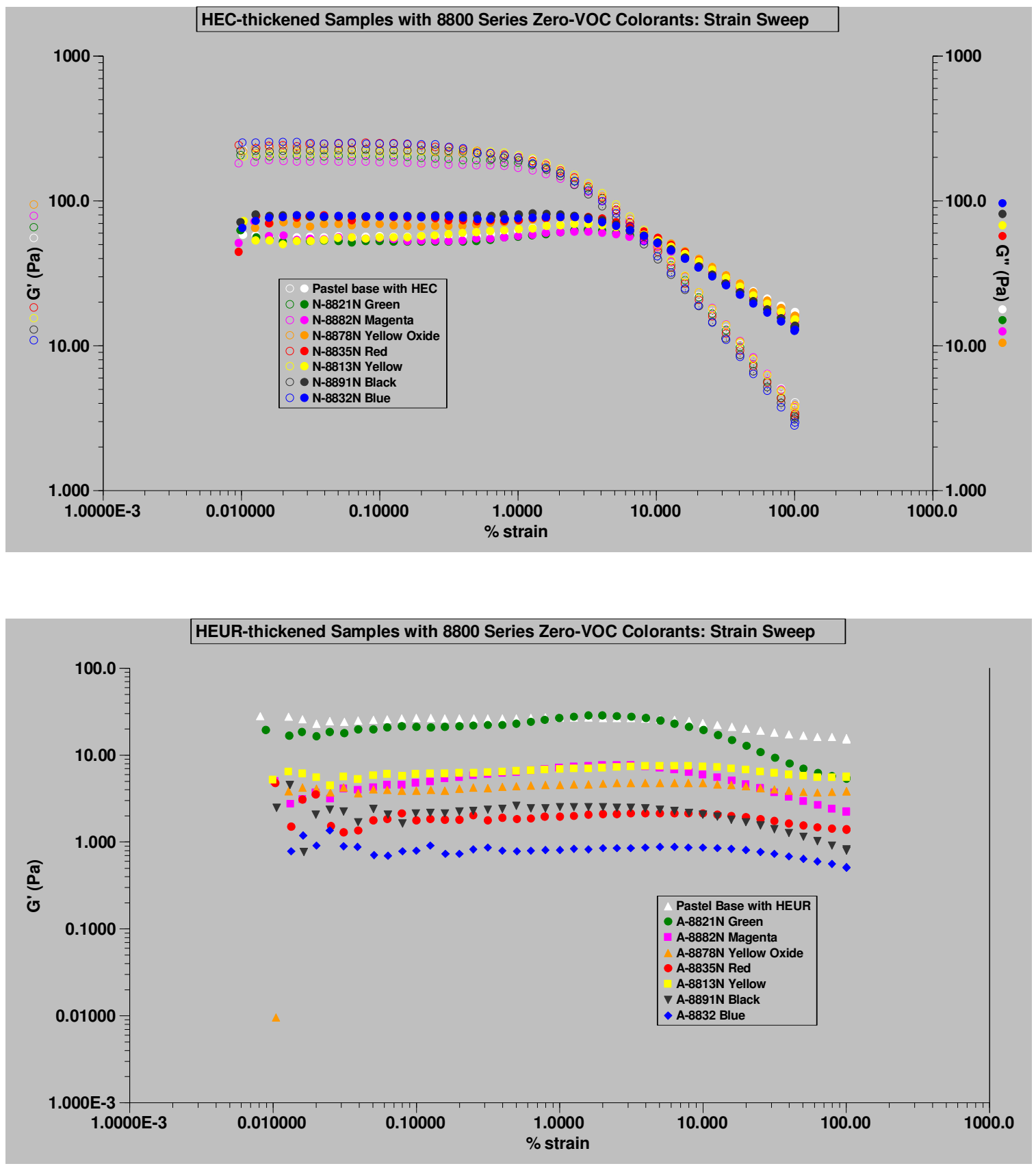


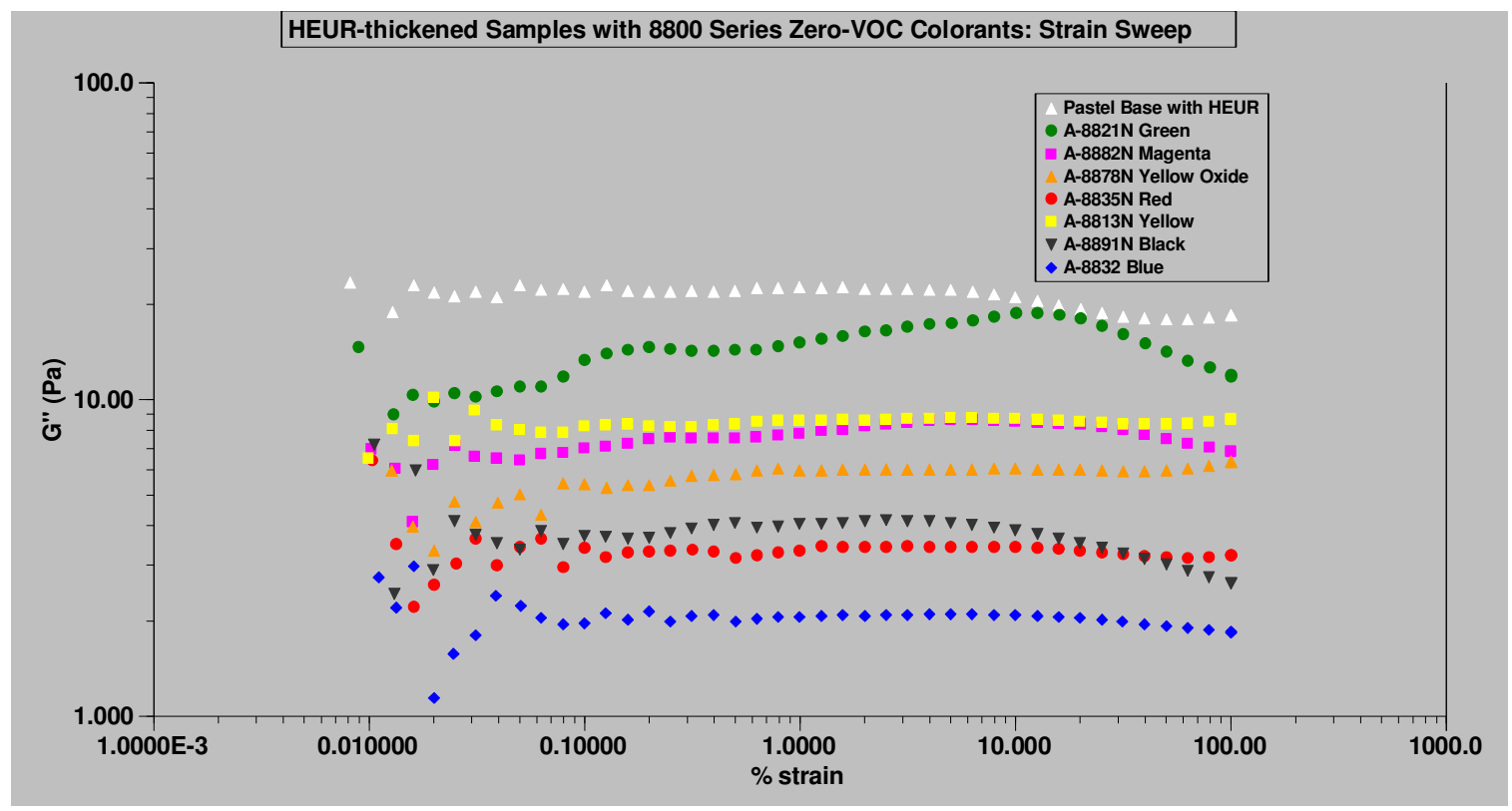




\section{Appendix 4}

\section{Strain Sweep of Pastel Samples by Thickener and Color}
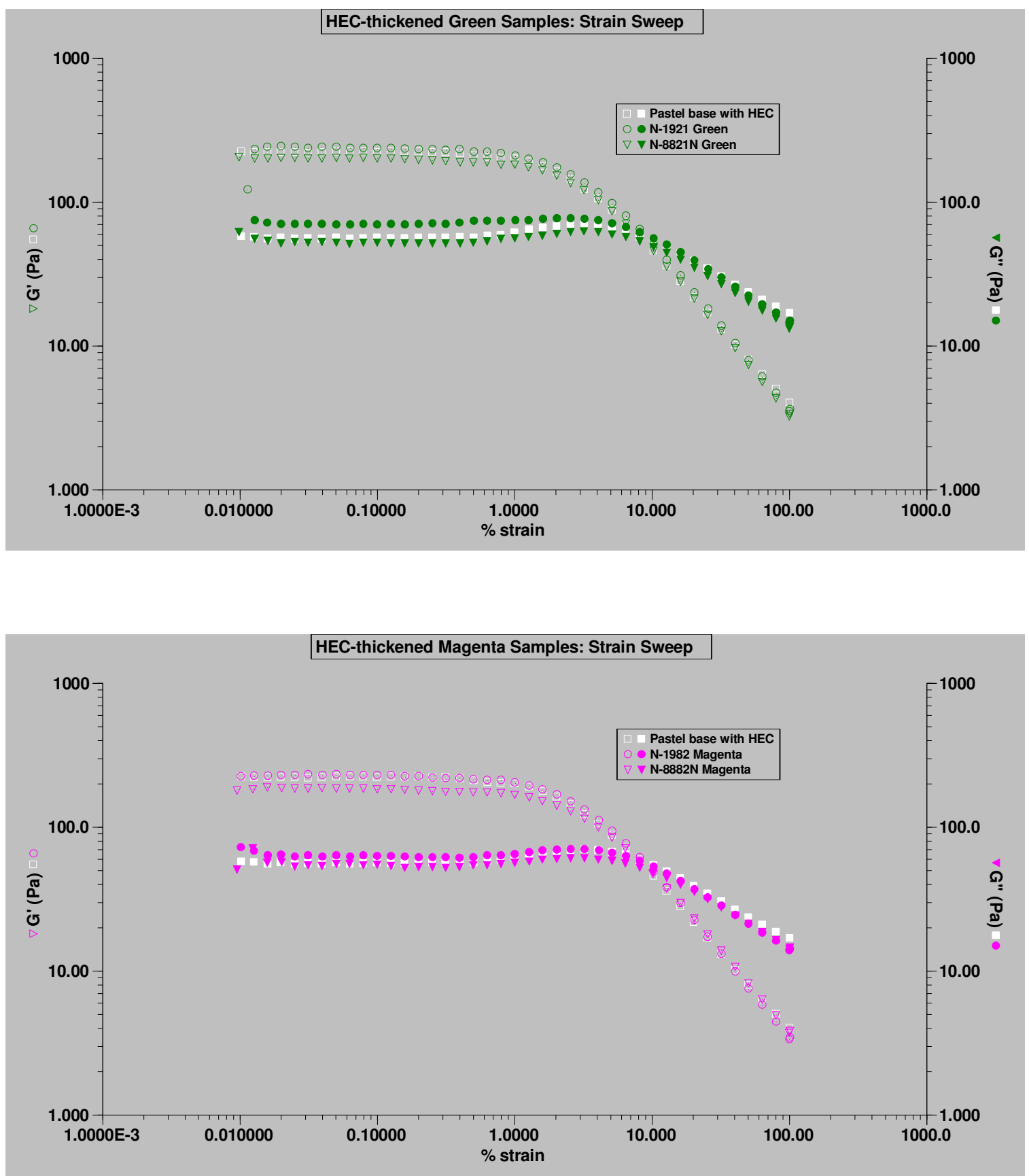

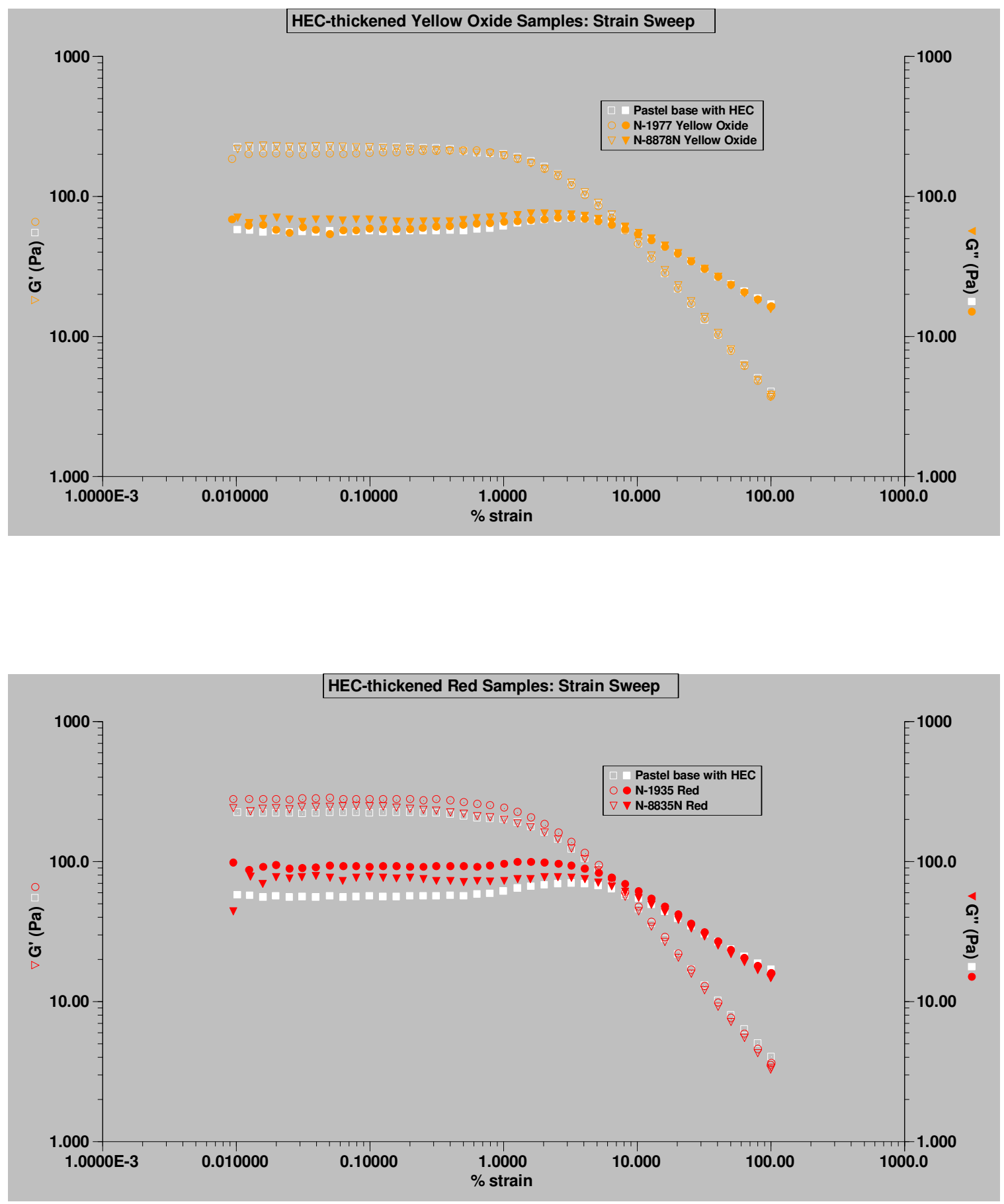

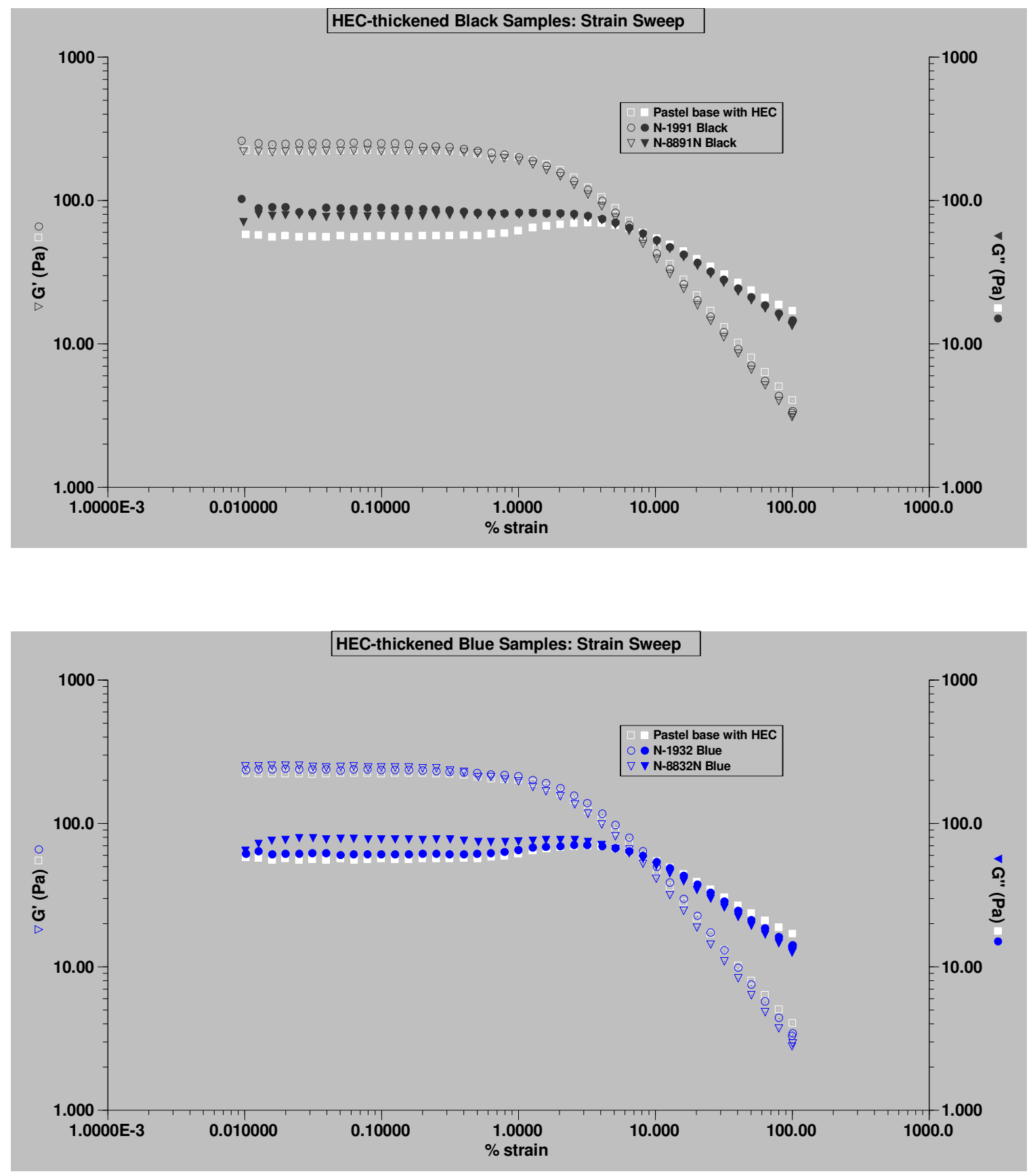


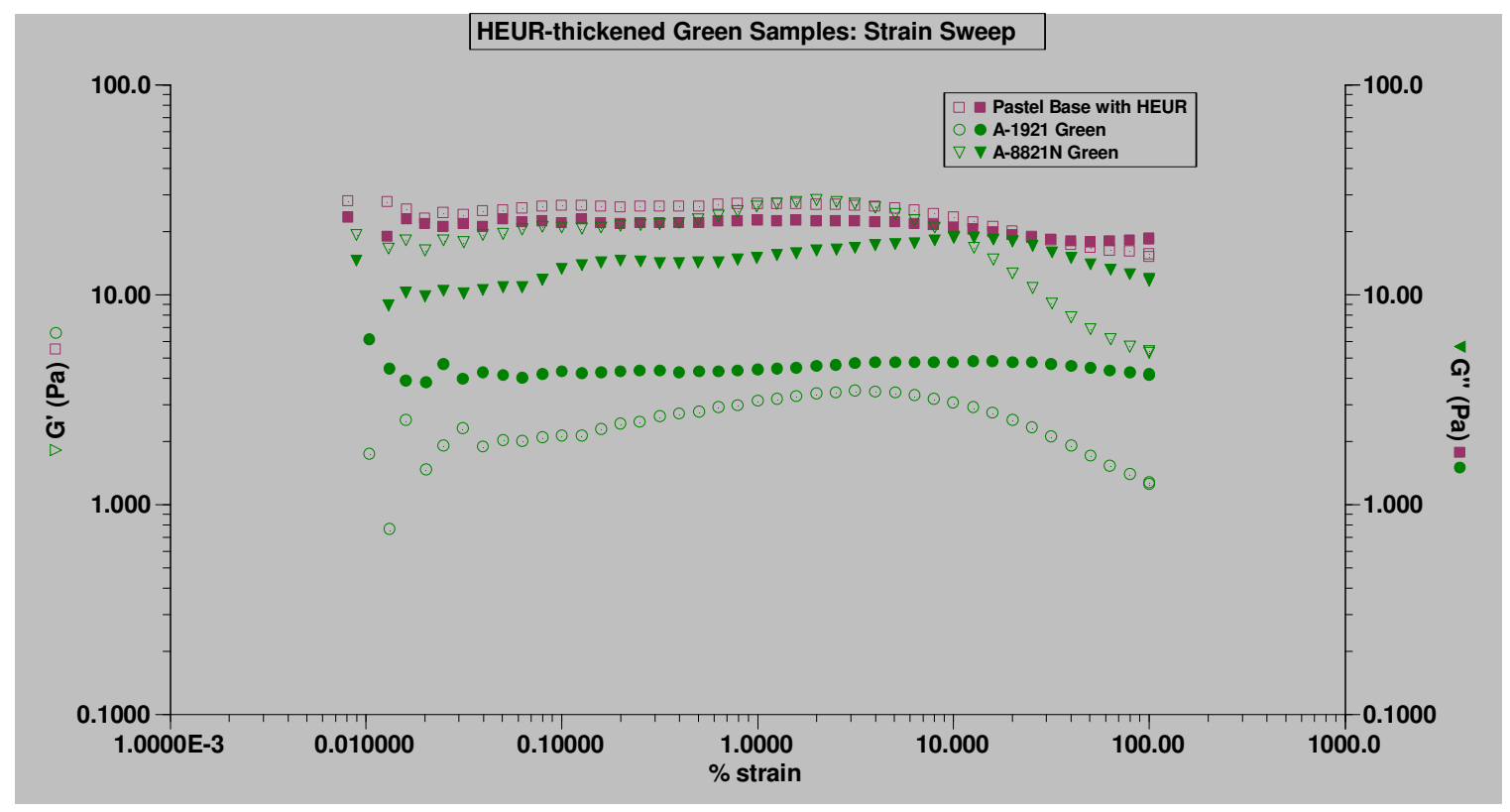

HEUR-thickened Magenta Samples: Strain Sweep

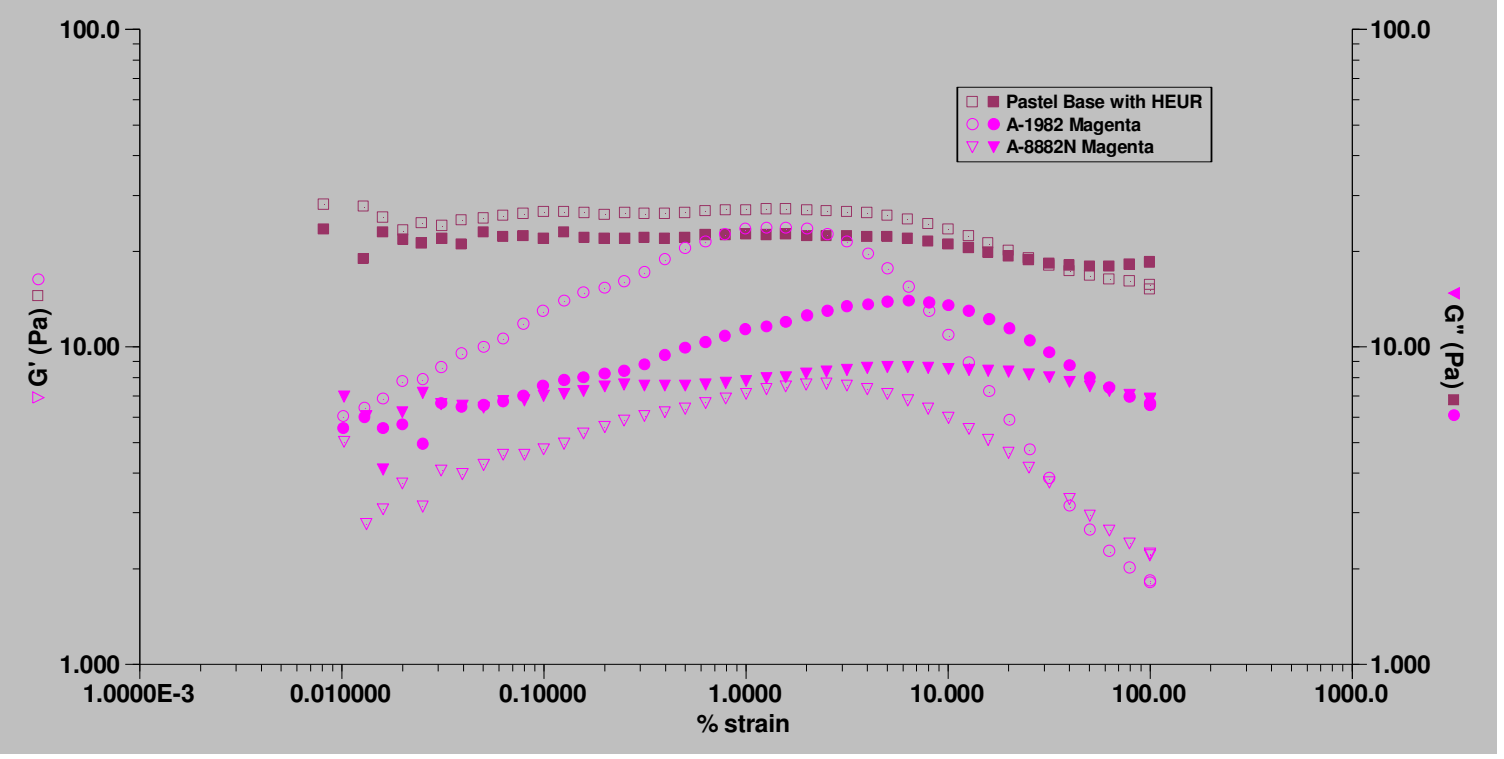



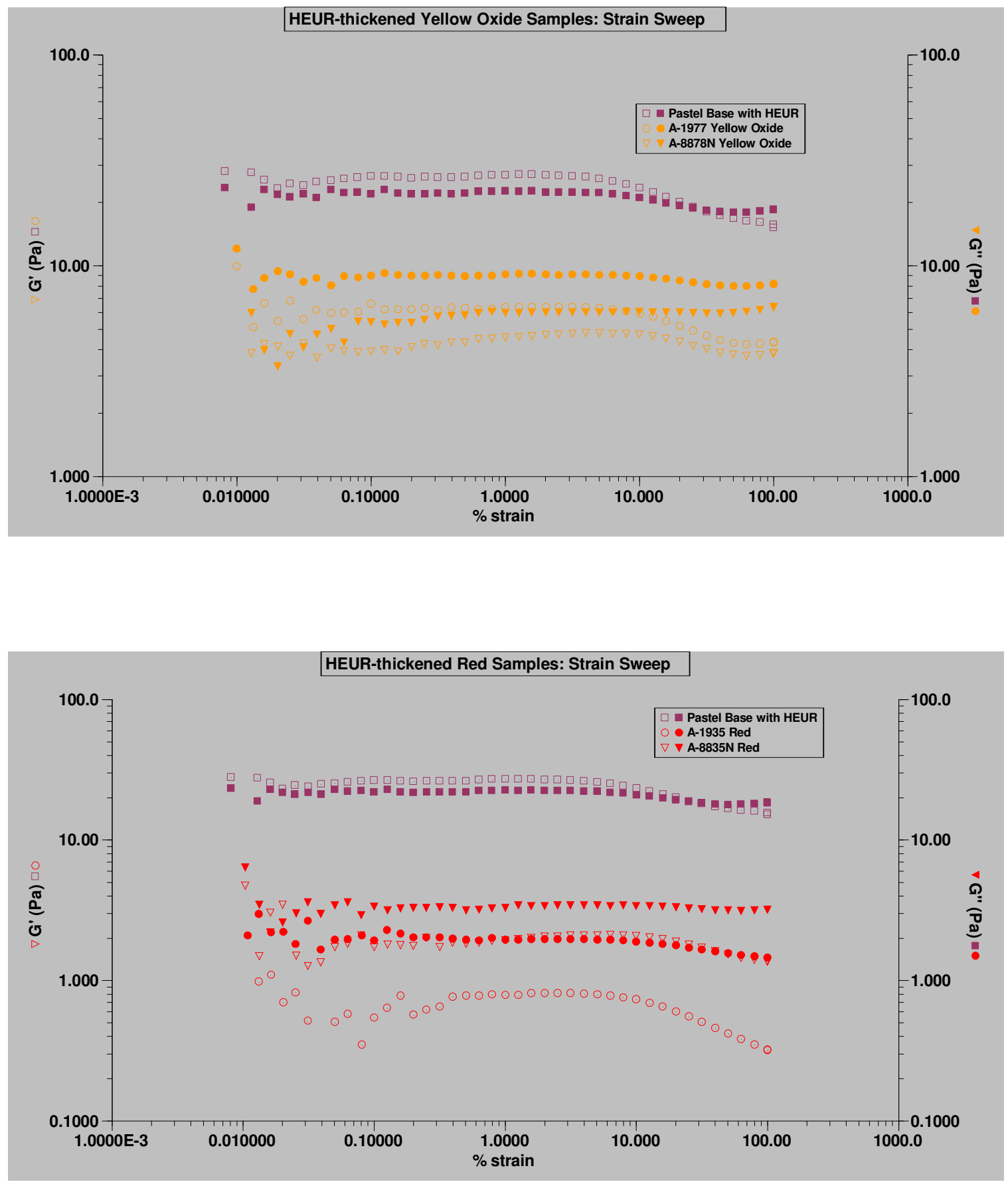

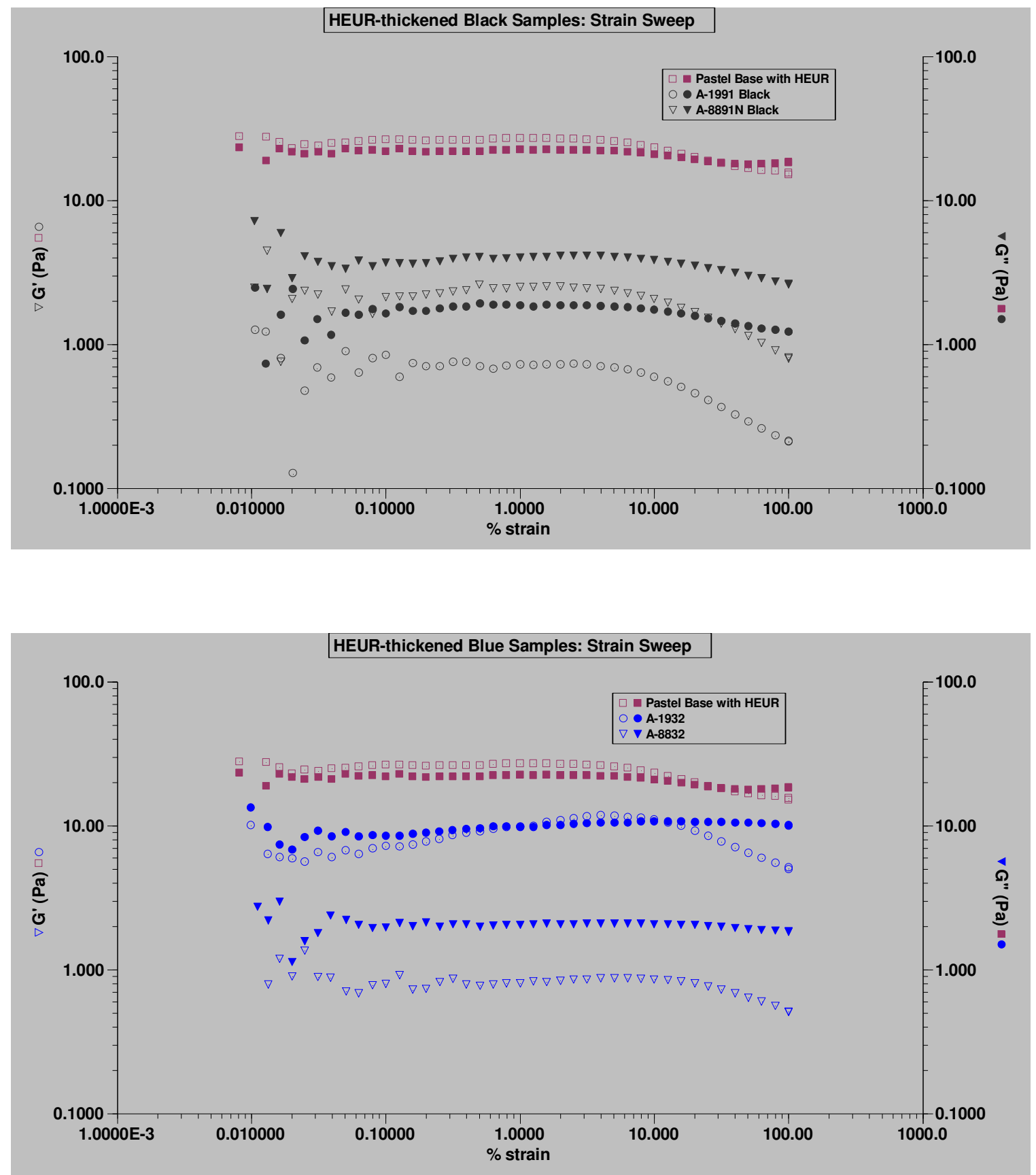


\section{Appendix 5}

Frequency Sweep of Pastel Samples Arranged by Thickener and Colorant Series
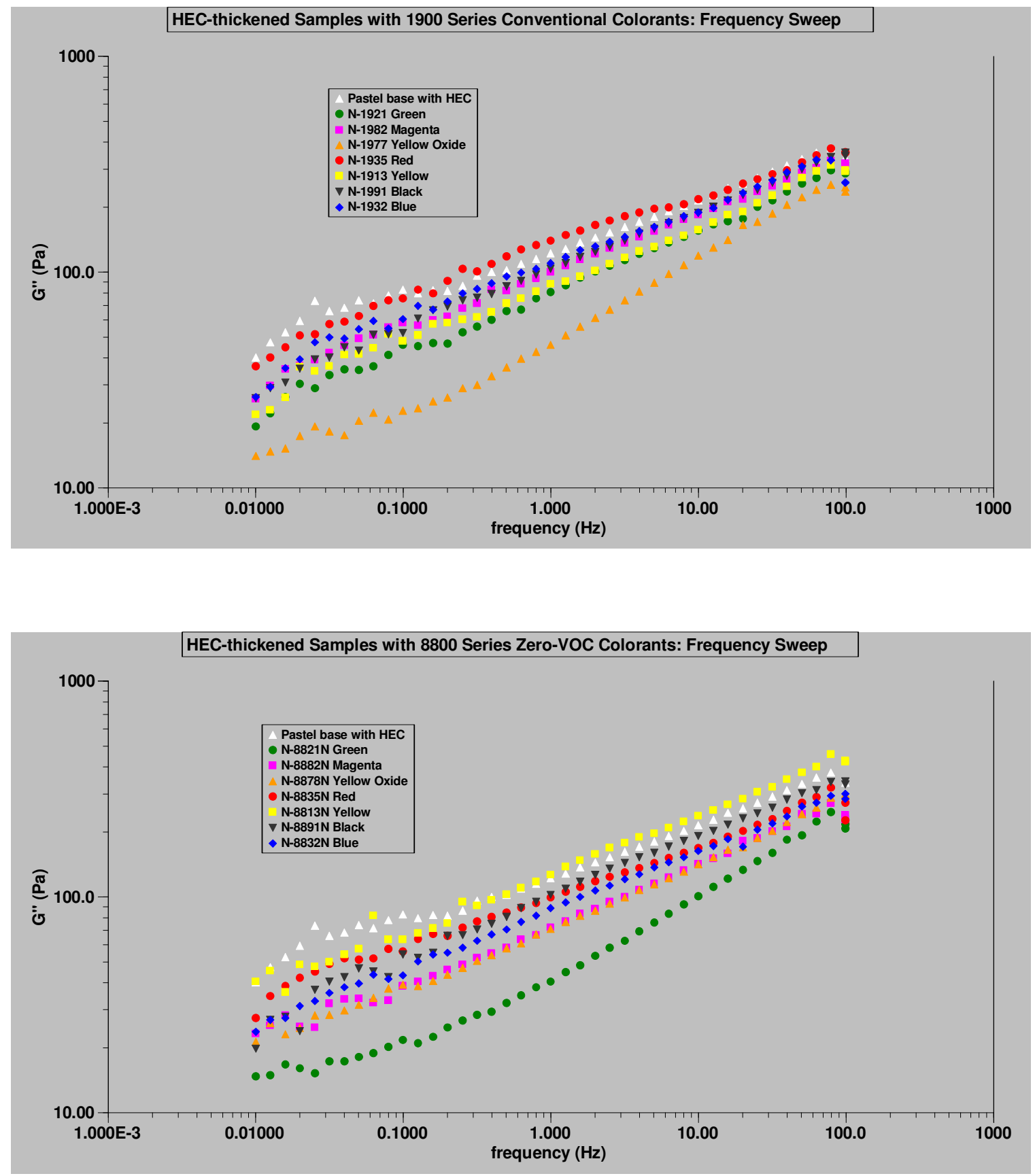

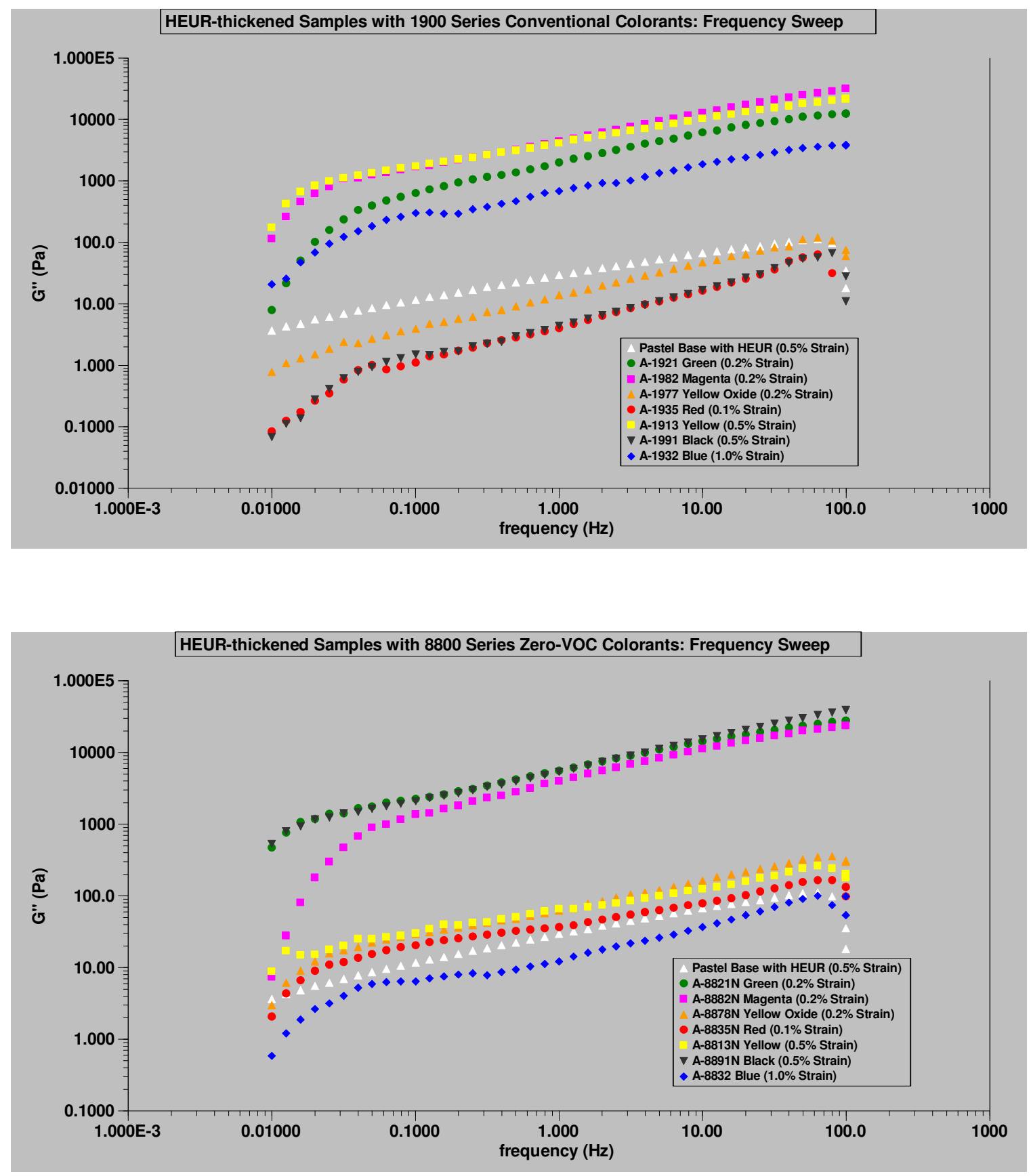


\section{Appendix 6}

Frequency Sweep of Pastel Samples Arranged by Thickener and Color
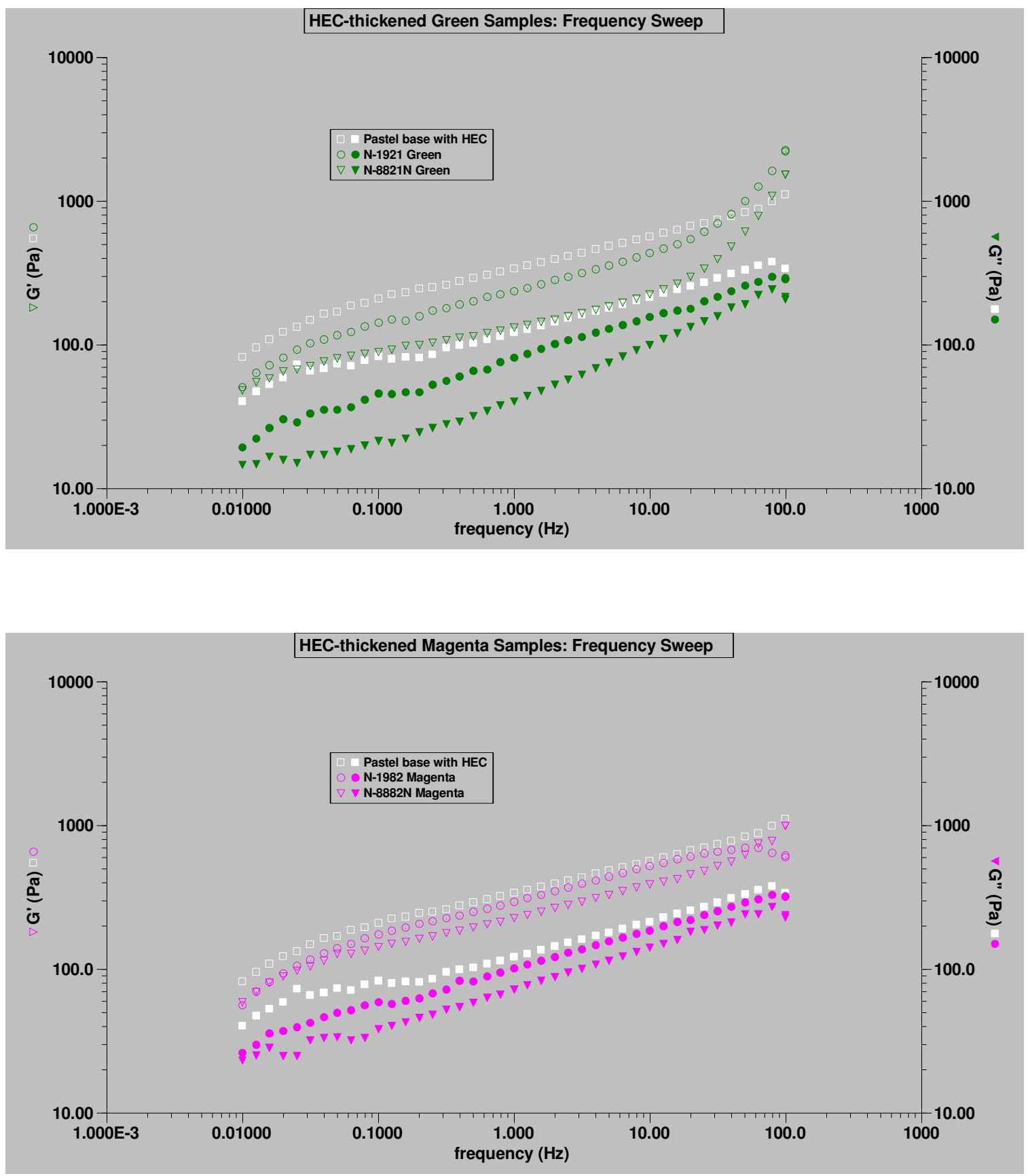

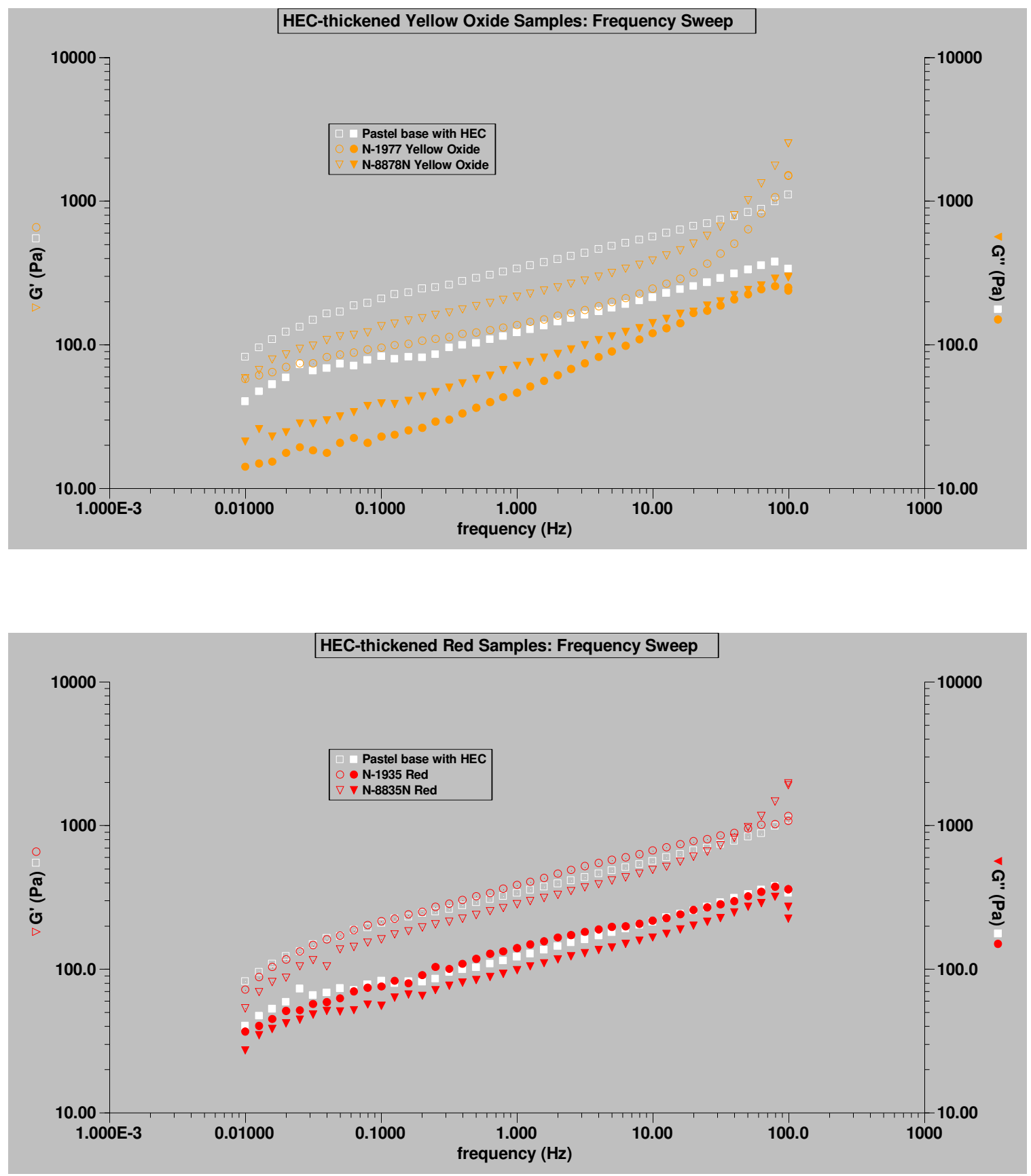

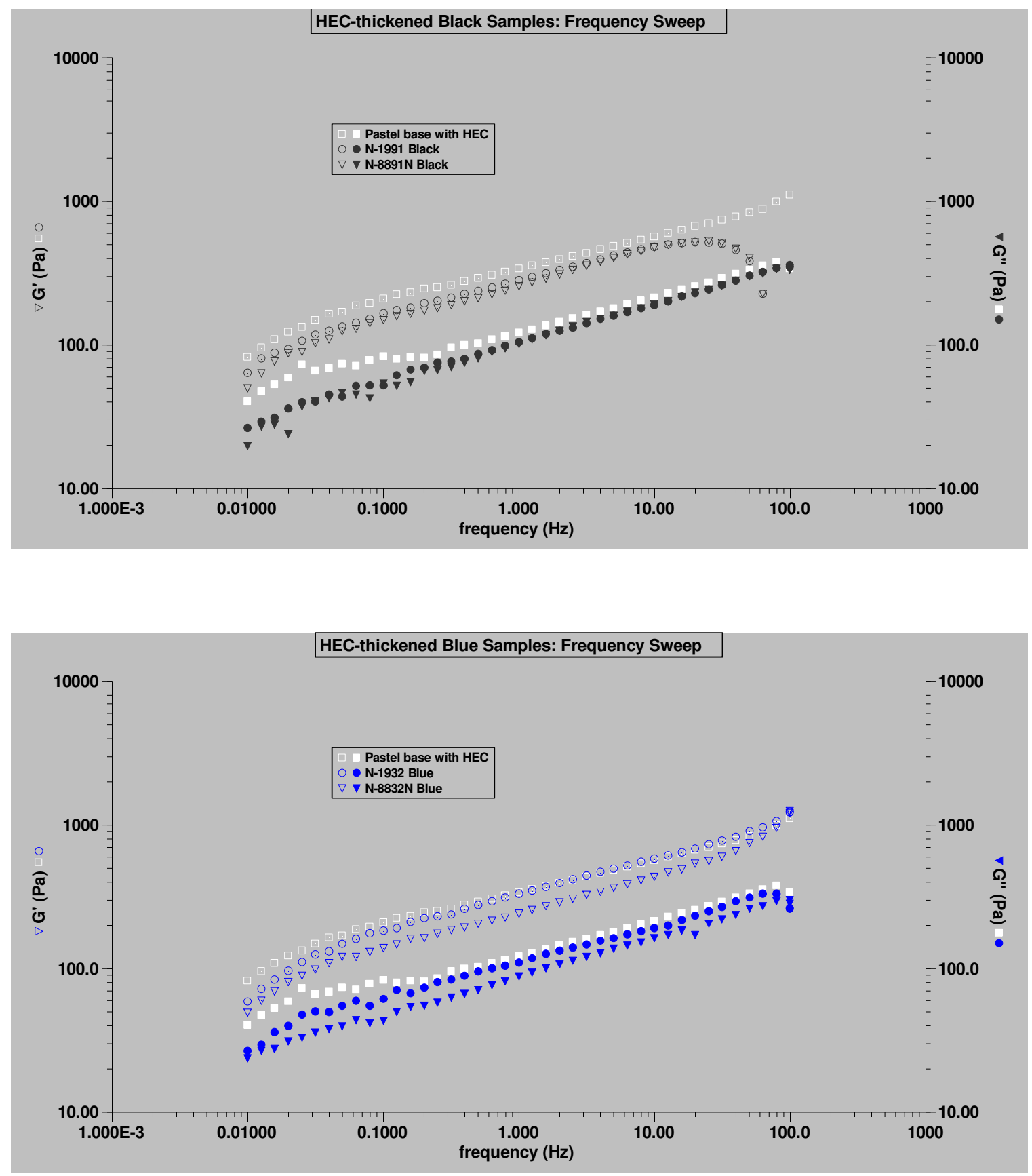

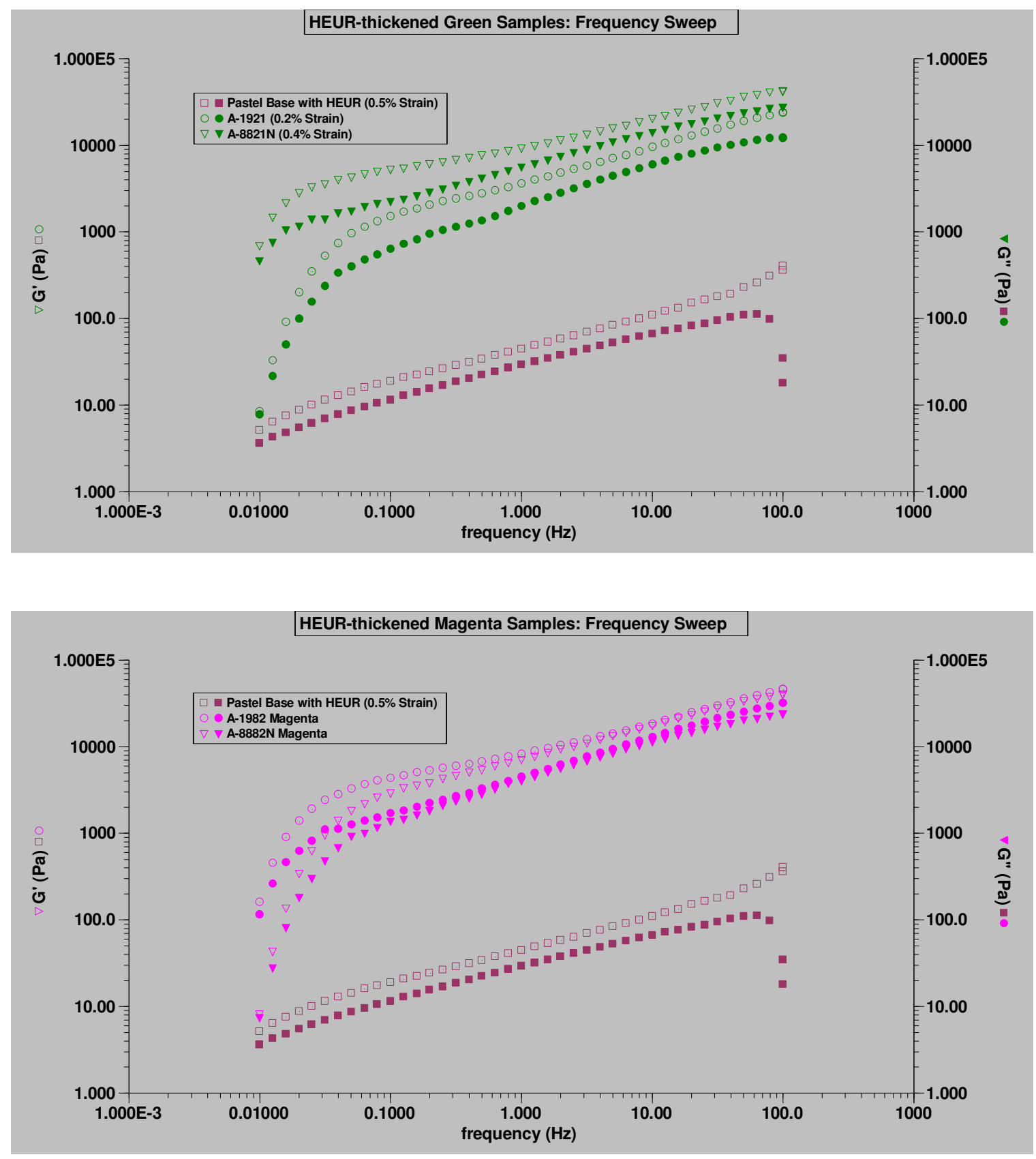

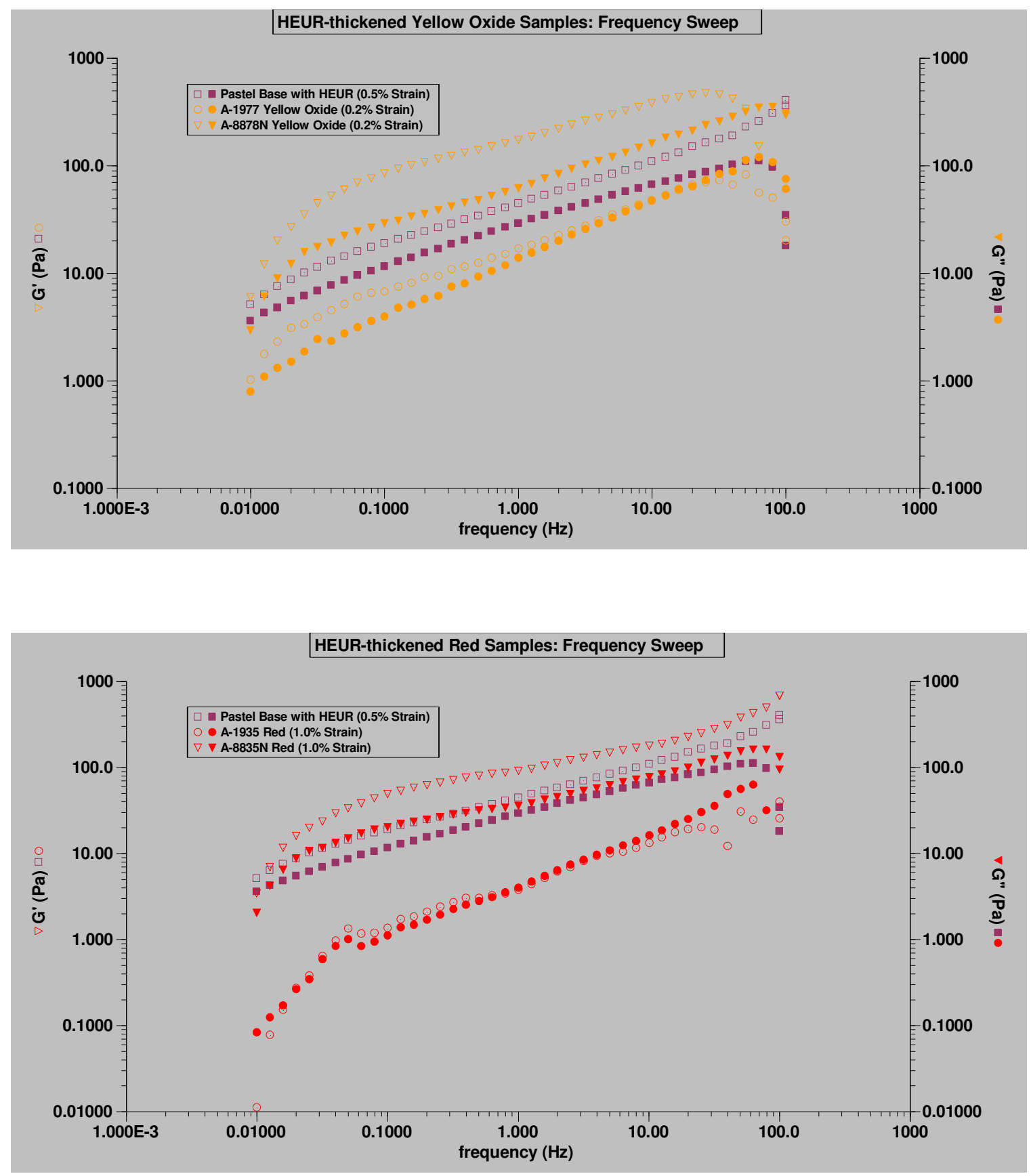

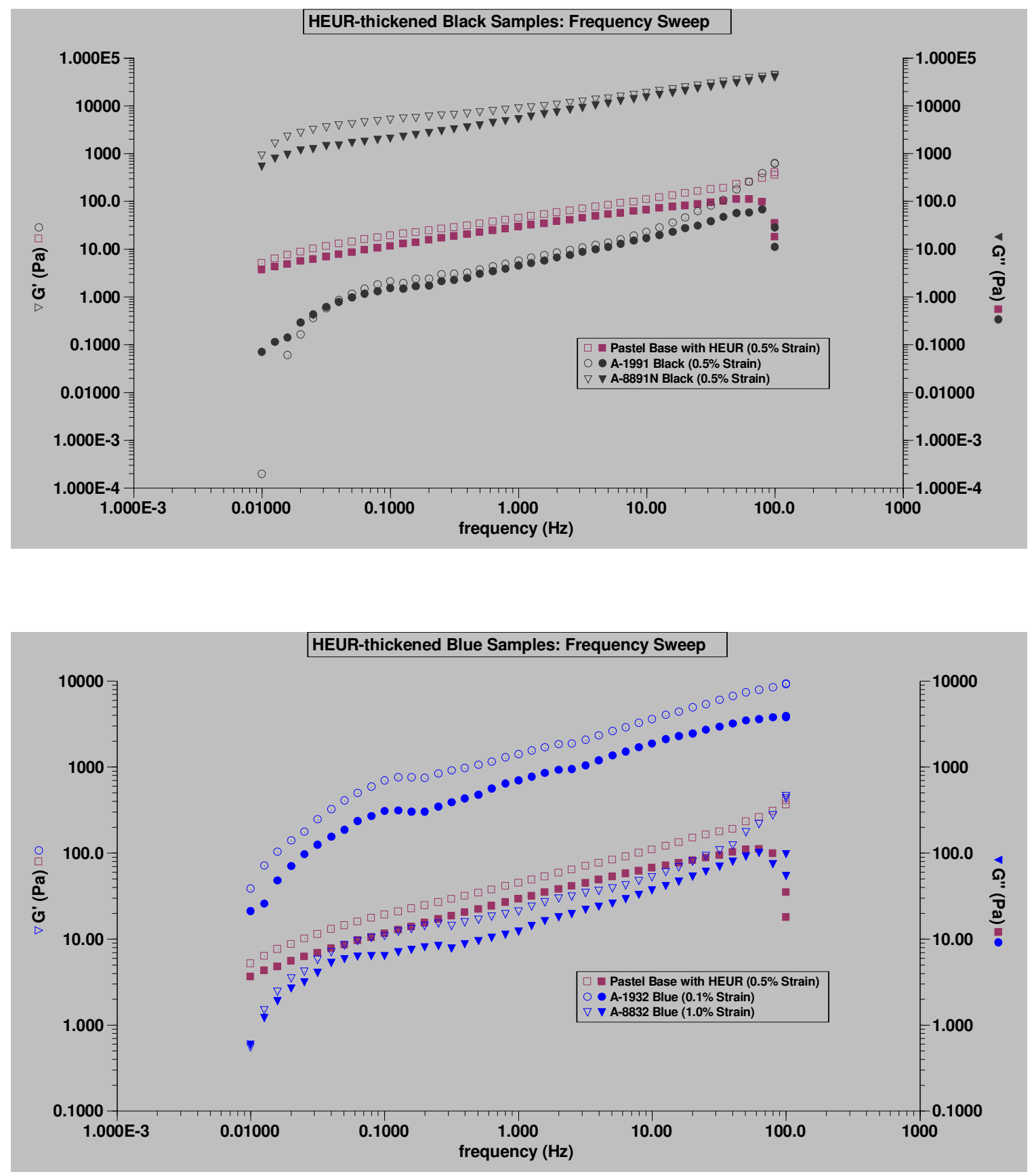


\section{Appendix 7}

Viscosity Profiles of Deeptone Samples Arranged by Thickener and Colorant Series
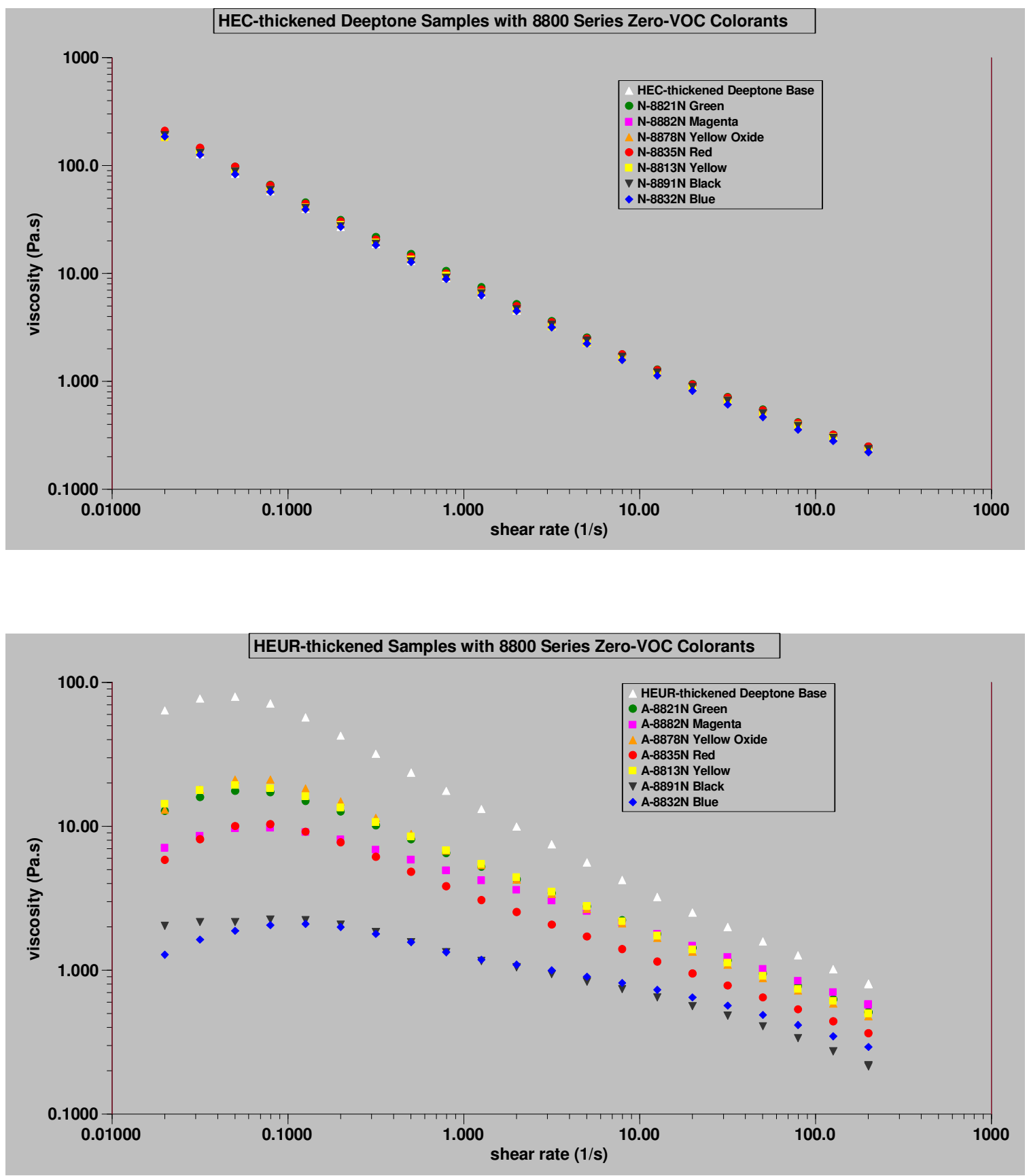


\section{Appendix 8}

\section{Viscosity Profiles of Deeptone Samples Arranged by Color}
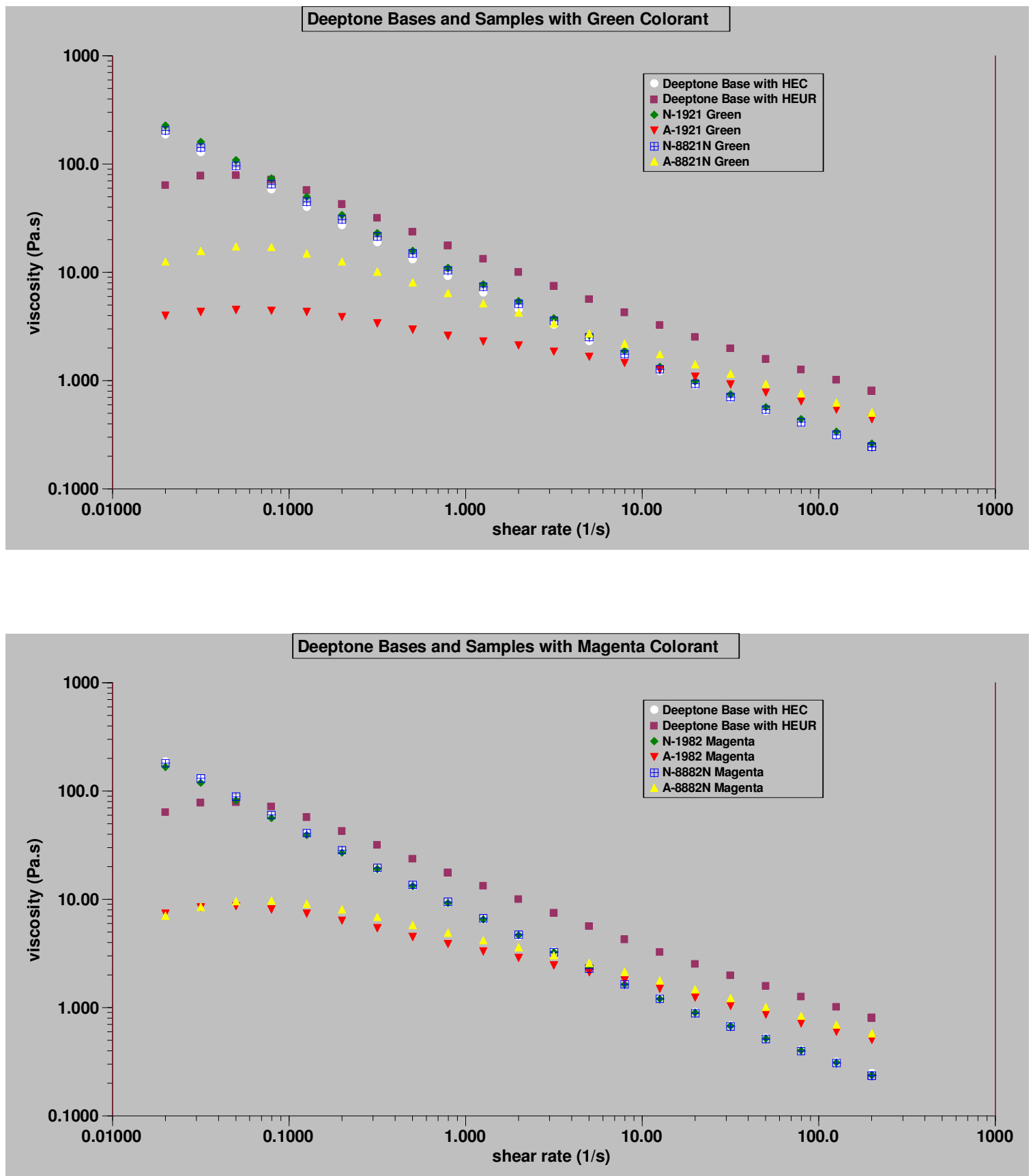

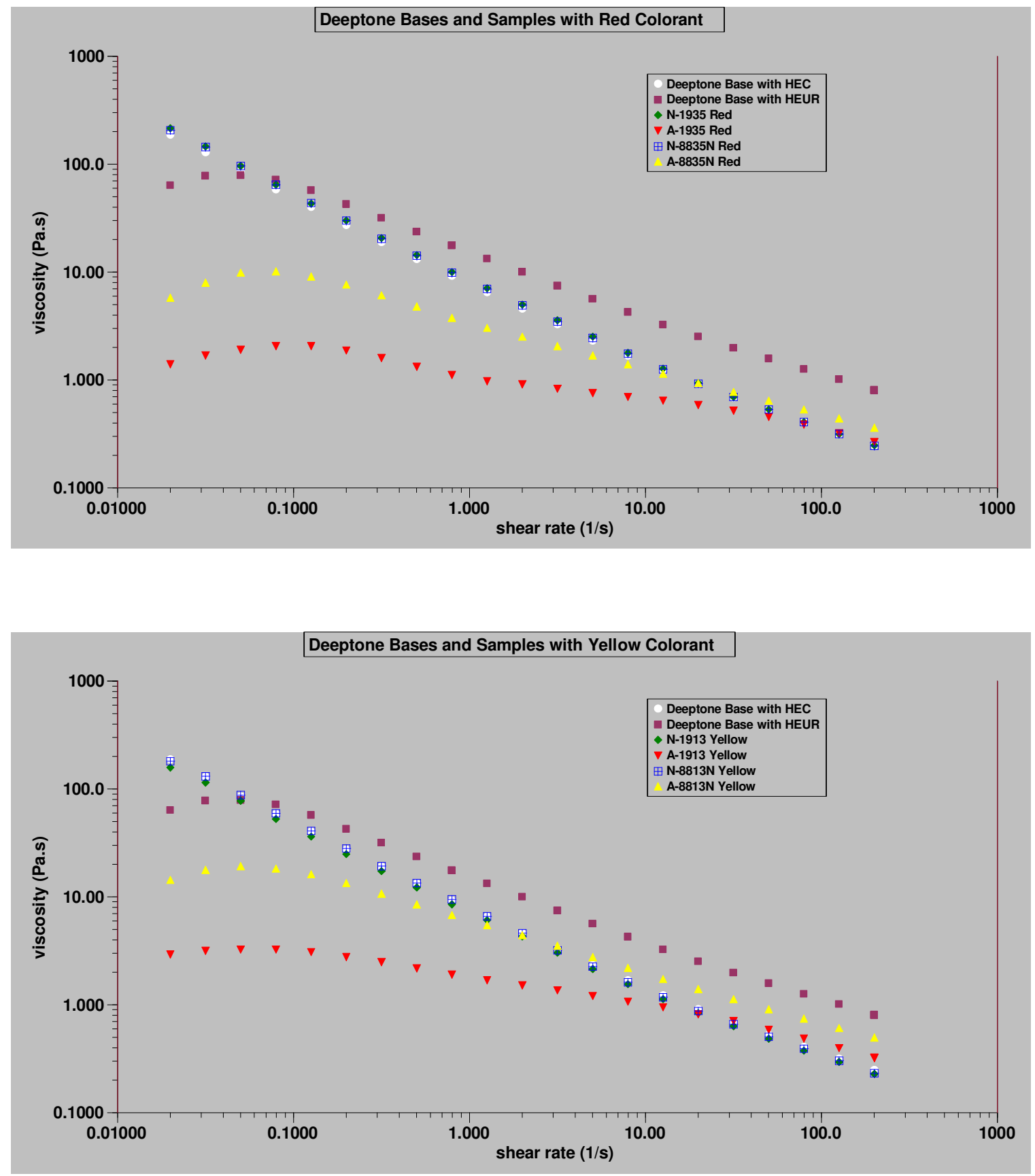


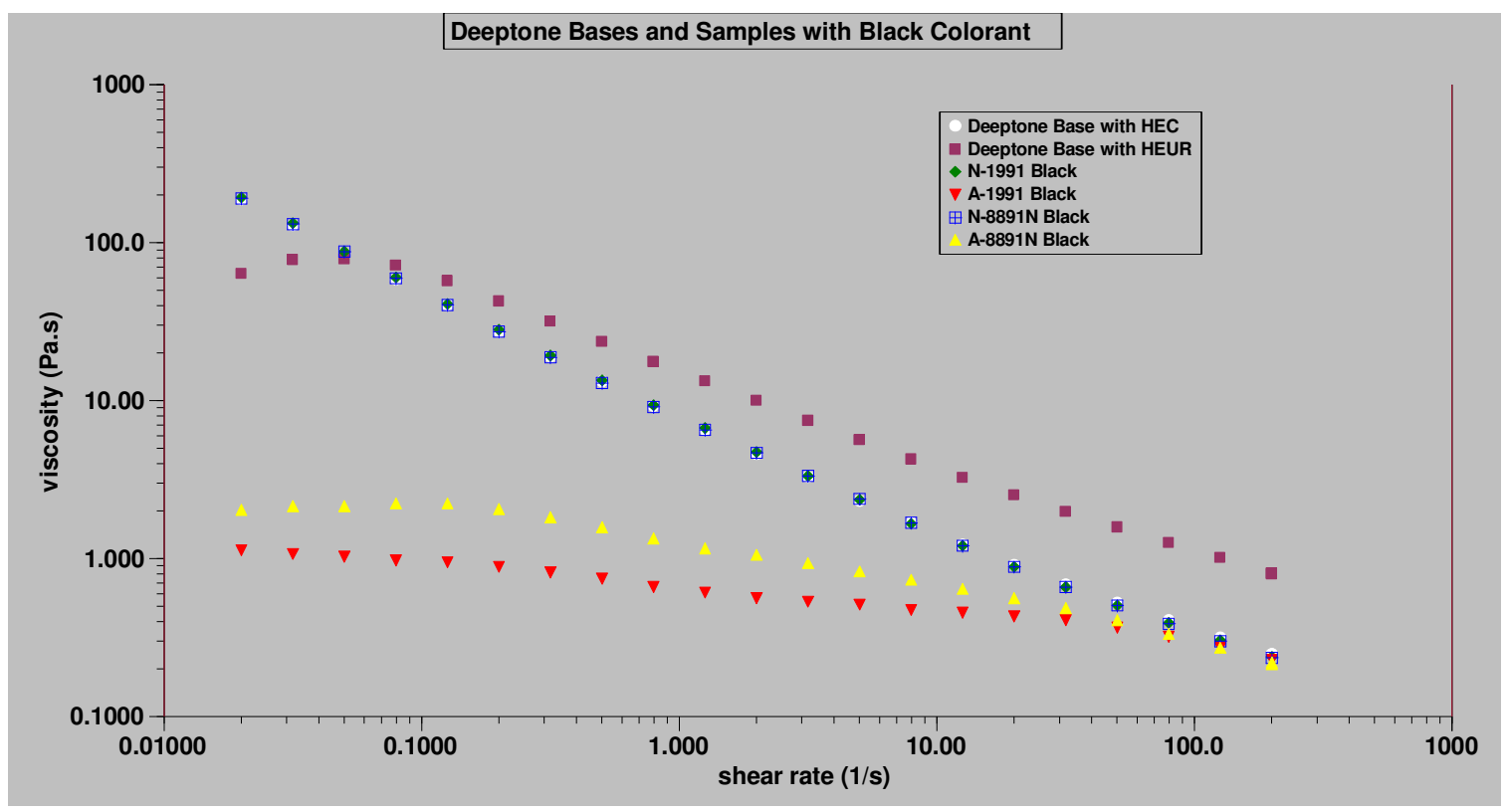




\section{Appendix 9}

\section{Strain Sweep of Deeptone Samples Arranged by Thickener and Colorant Series}
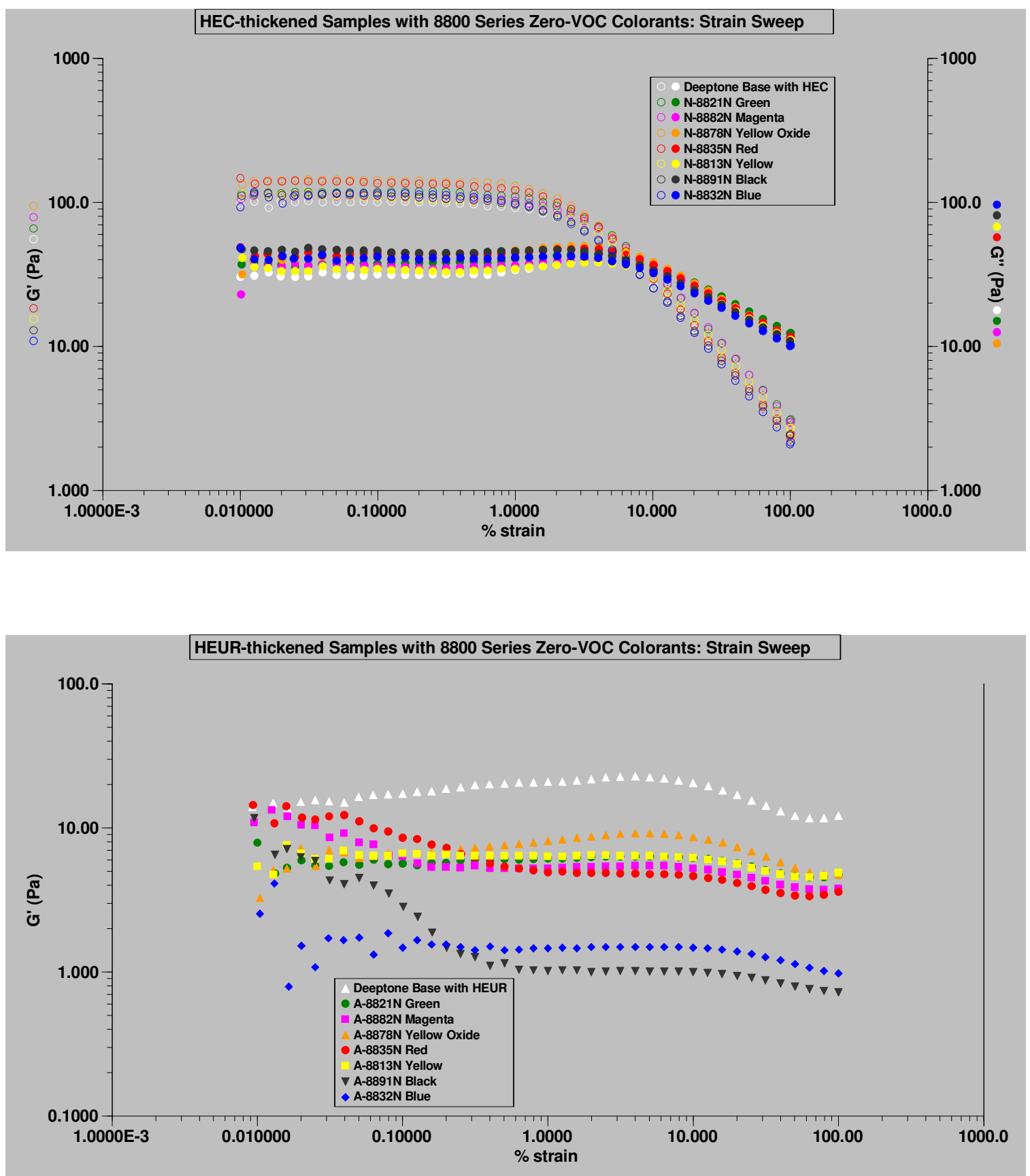


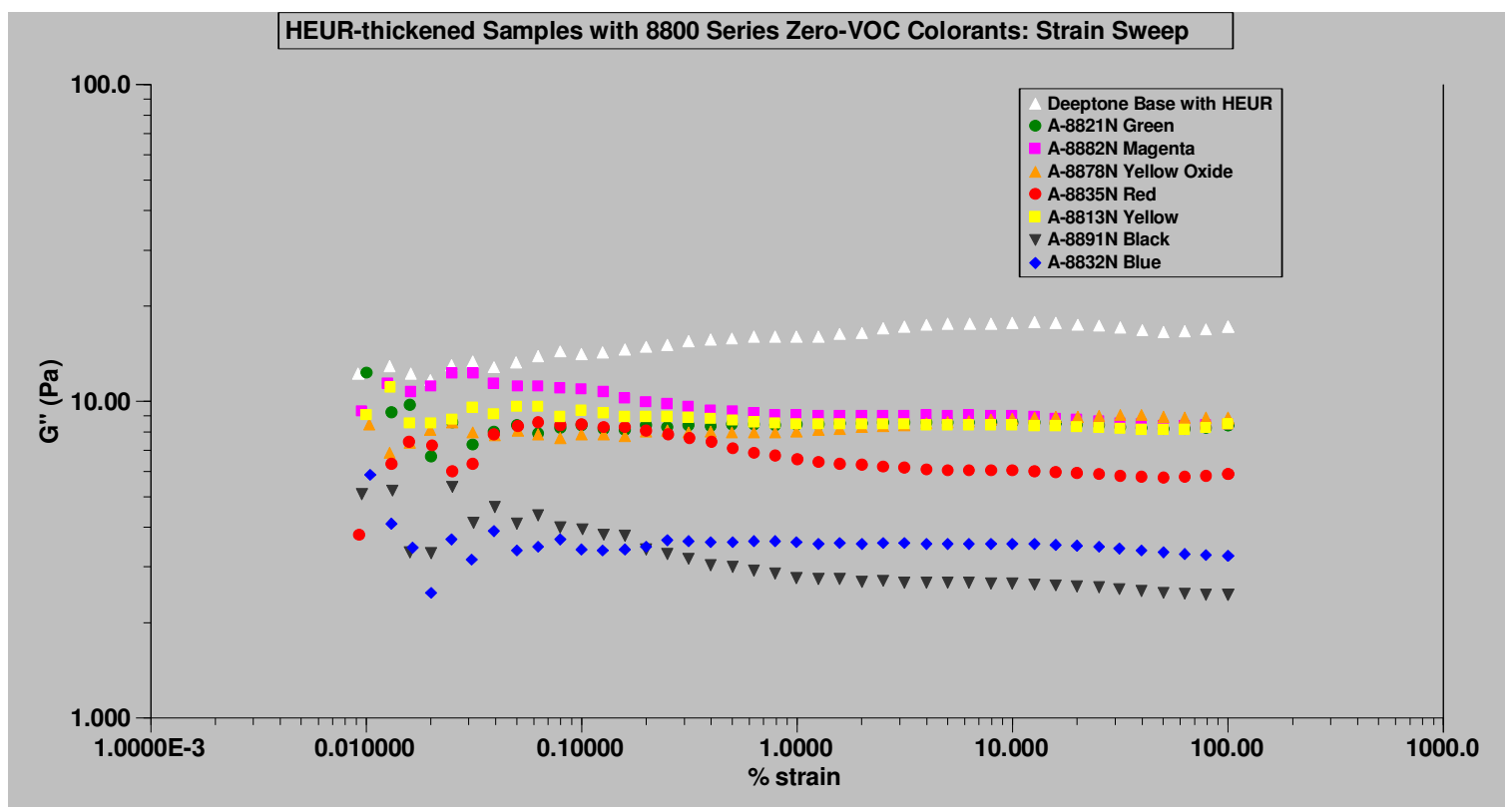




\section{Appendix 10}

\section{Strain Sweep of Deeptone Samples Arranged by Thickener and Color}
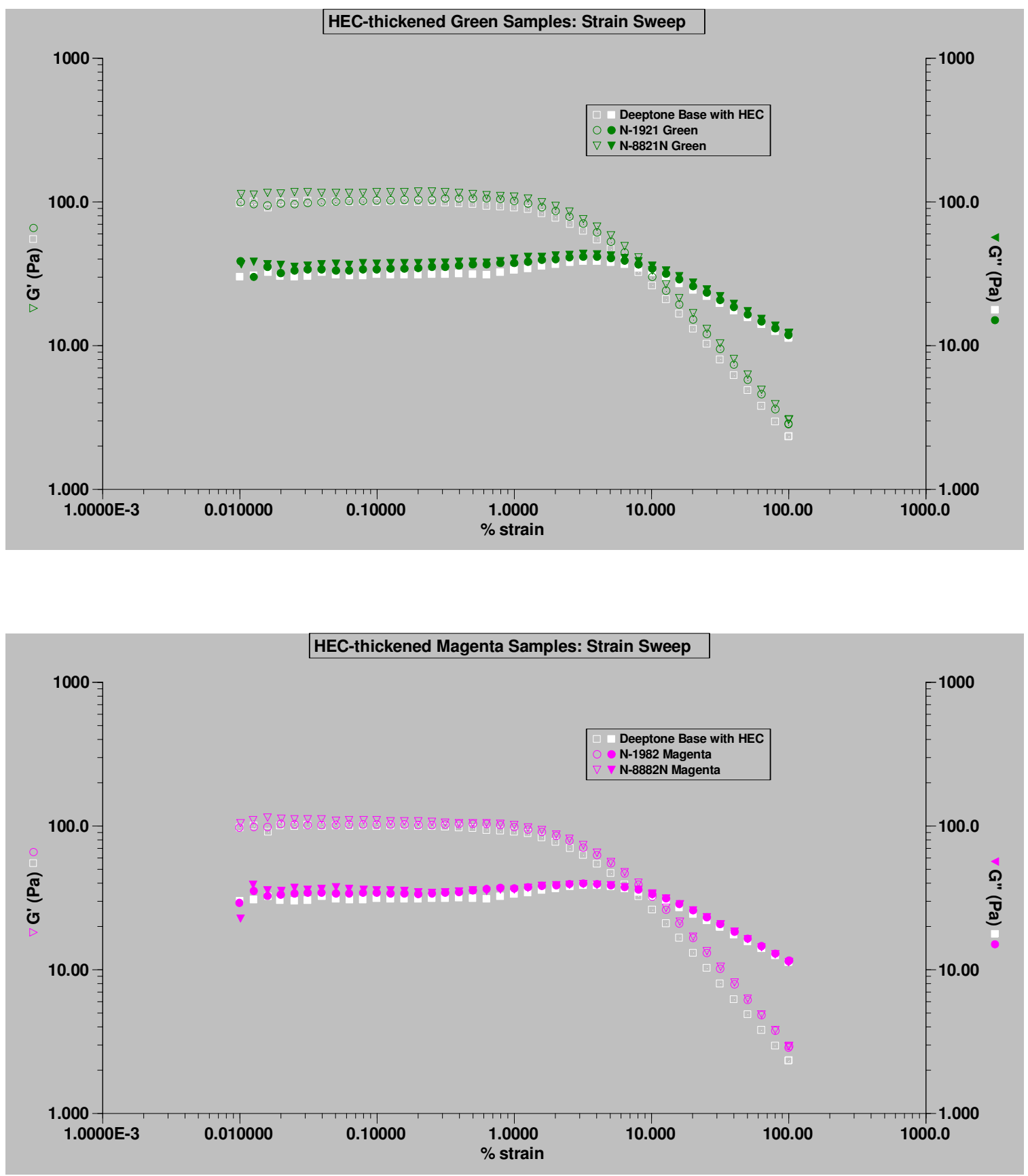
HEC-thickened Red Samples: Strain Sweep

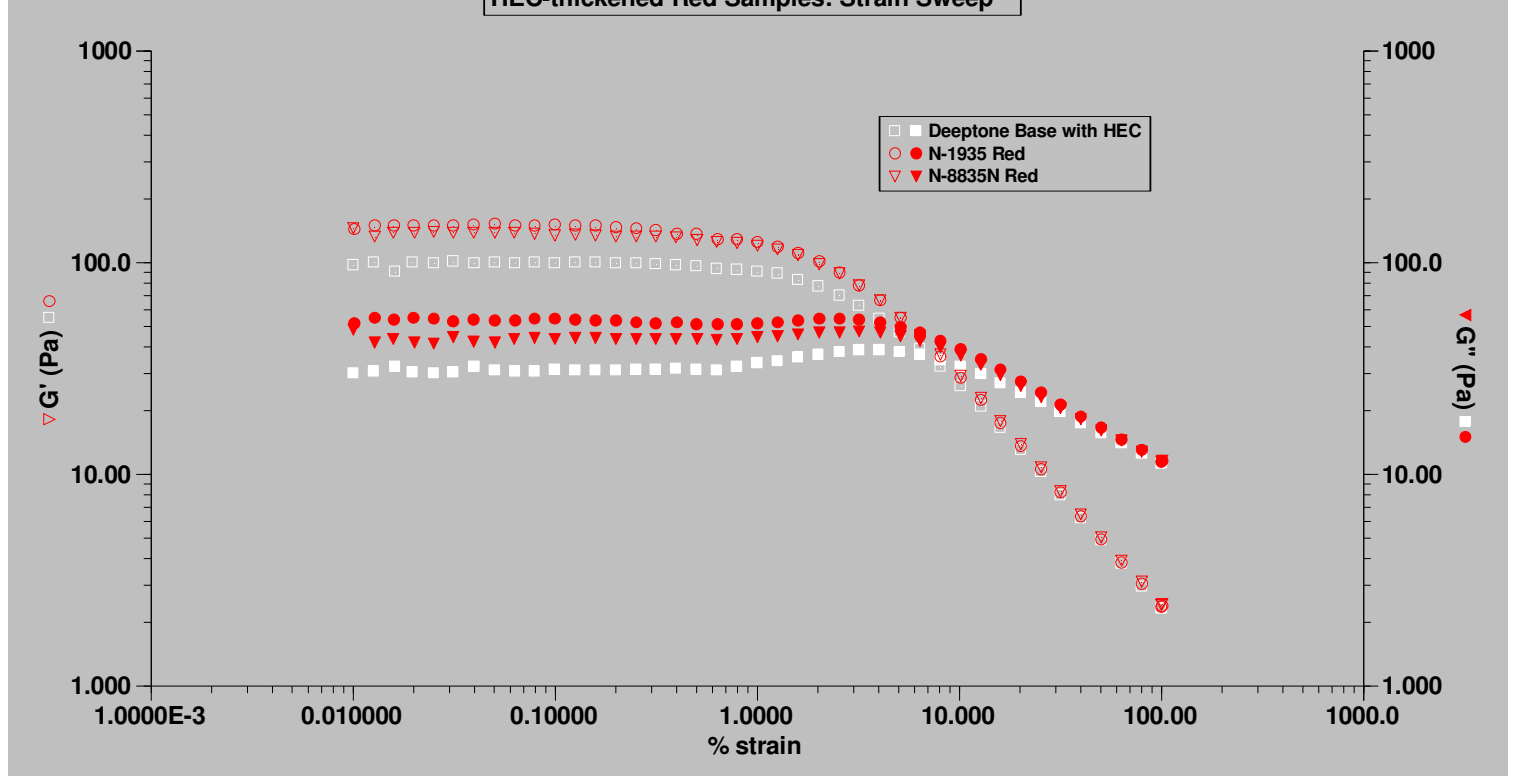

HEC-thickened Yellow Samples: Strain Sweep

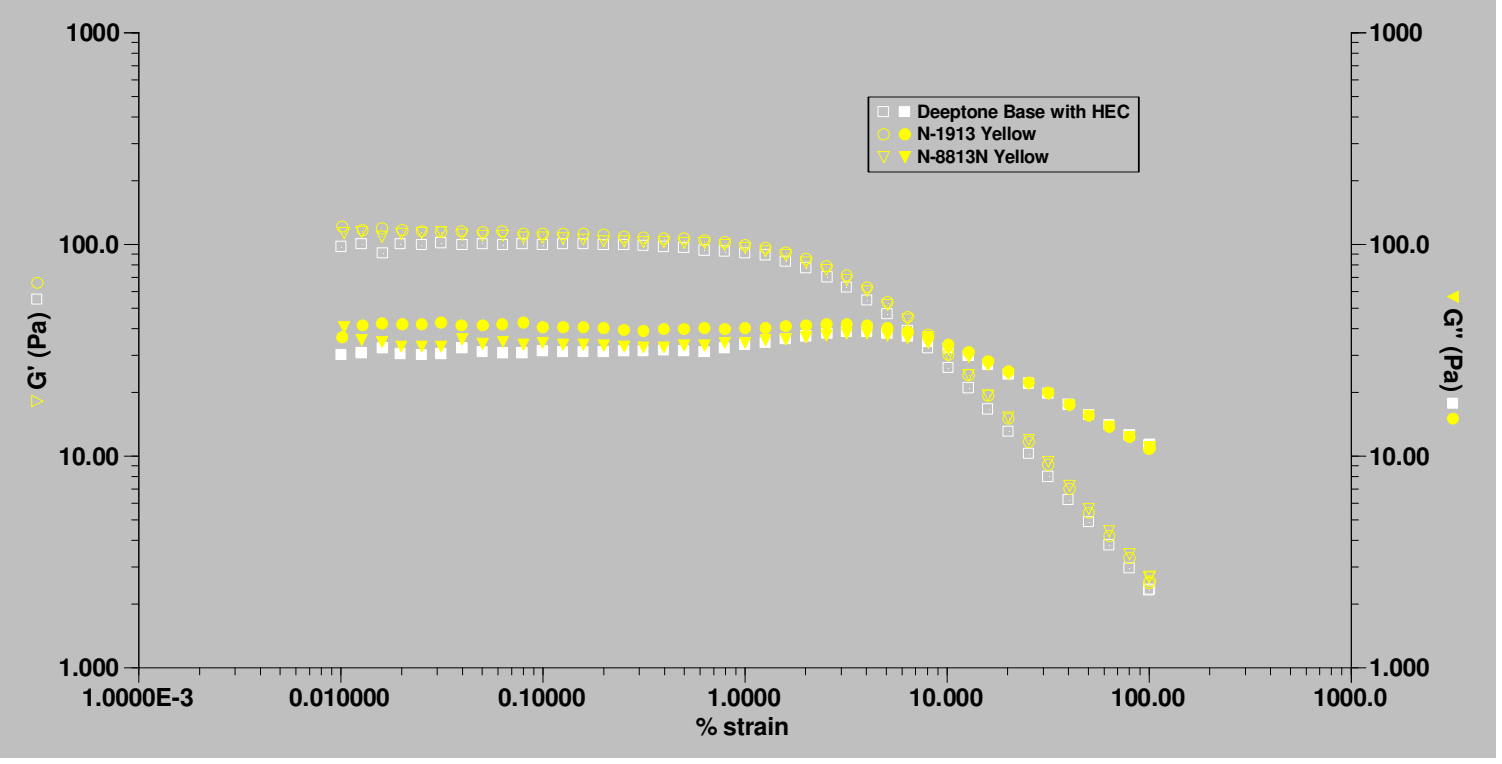


HEC-thickened Blue Samples: Strain Sweep
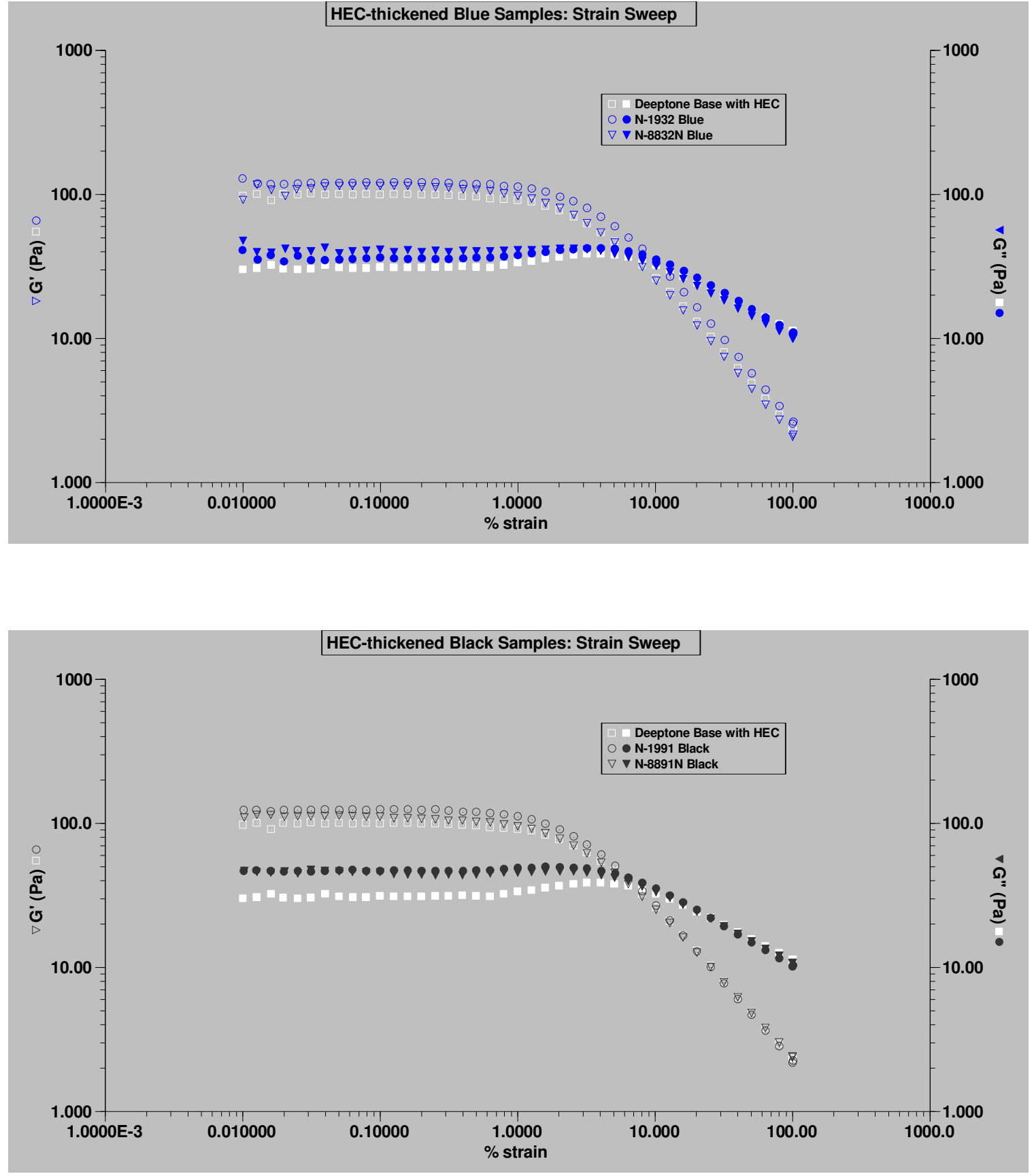

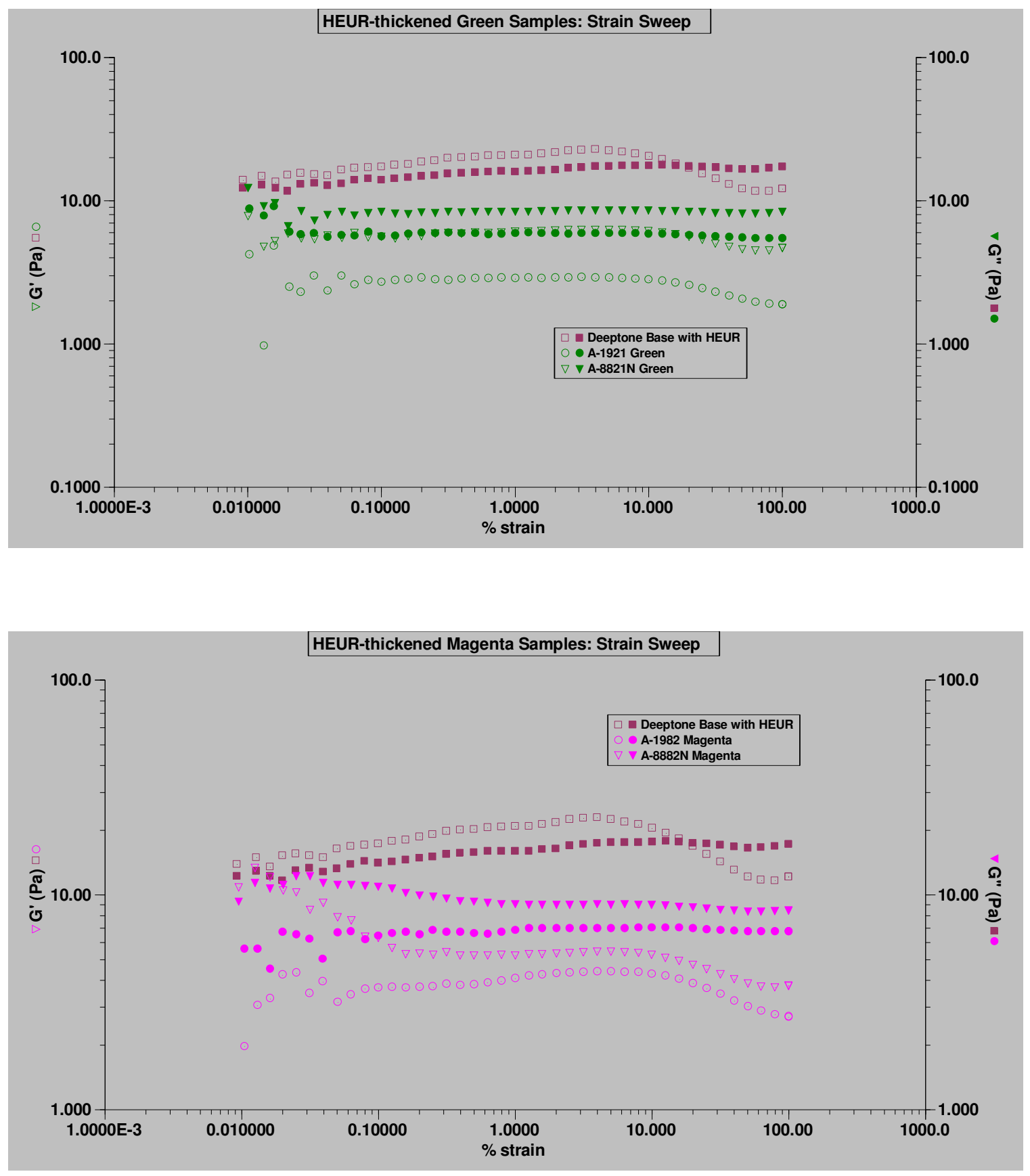

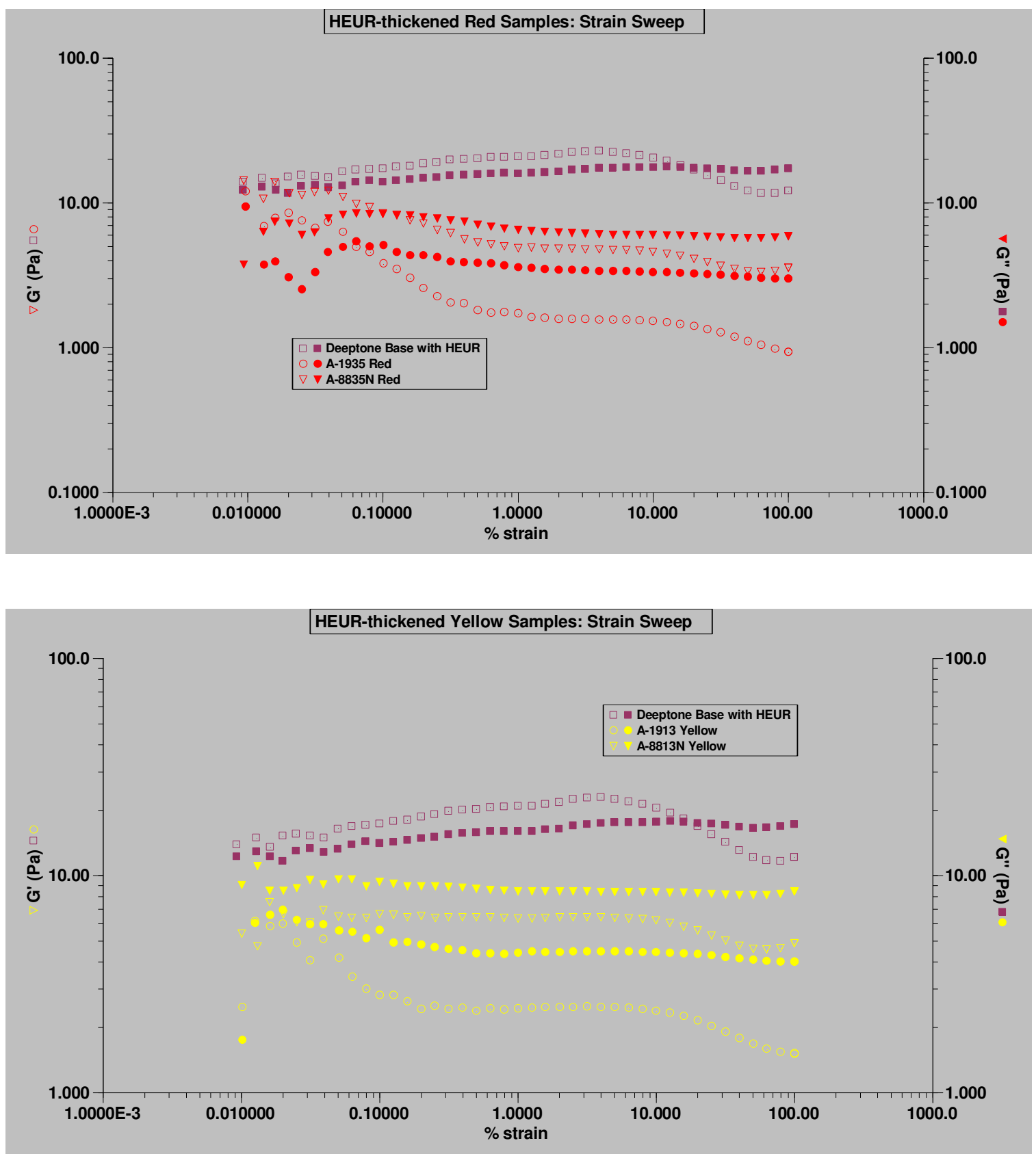

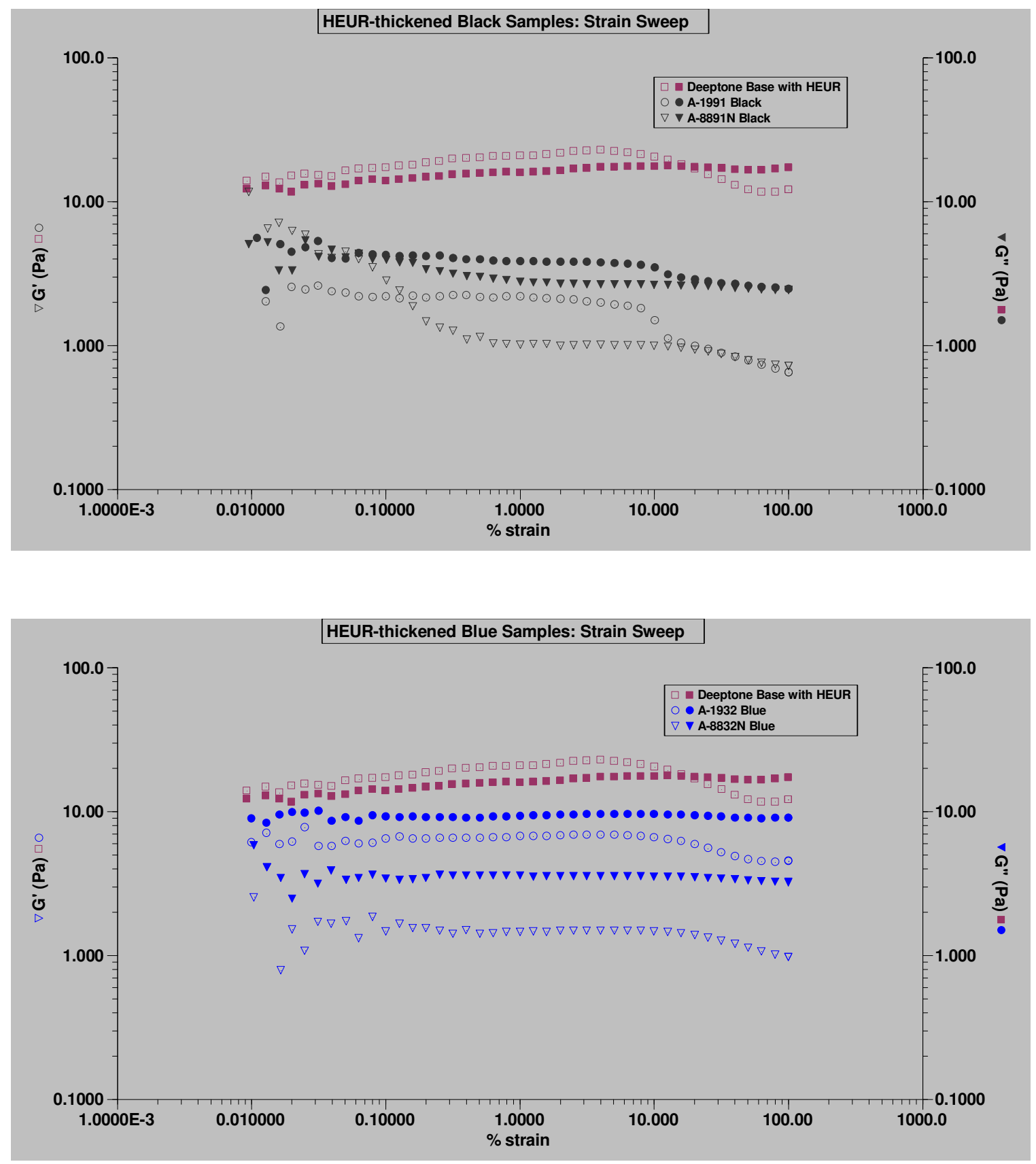


\section{Appendix 11}

Frequency Sweep of Deeptone Samples Arranged by Thickener and Colorant Series
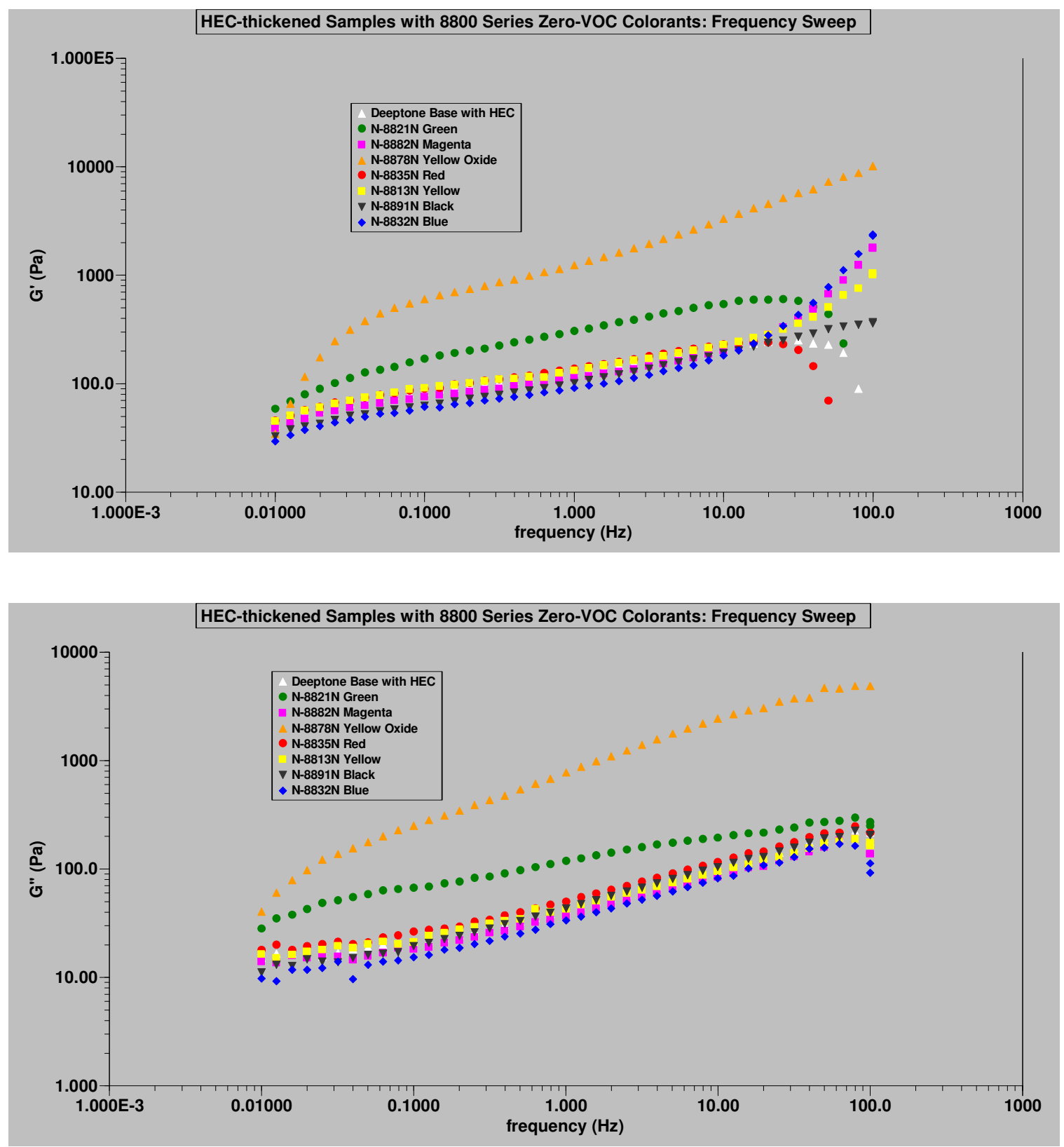

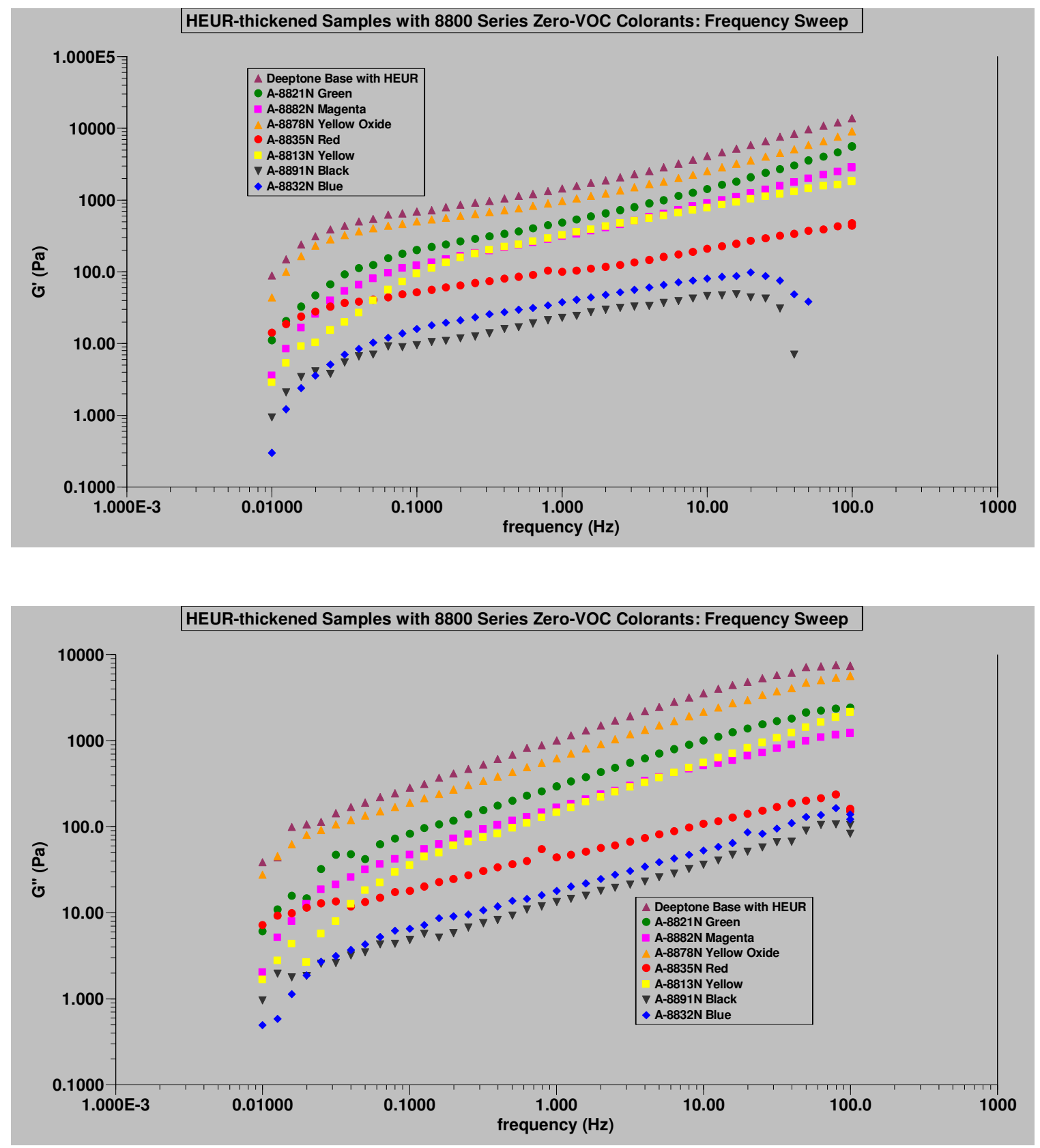


\section{Appendix 12}

Frequency Sweep of Deeptone Samples Arranged by Color
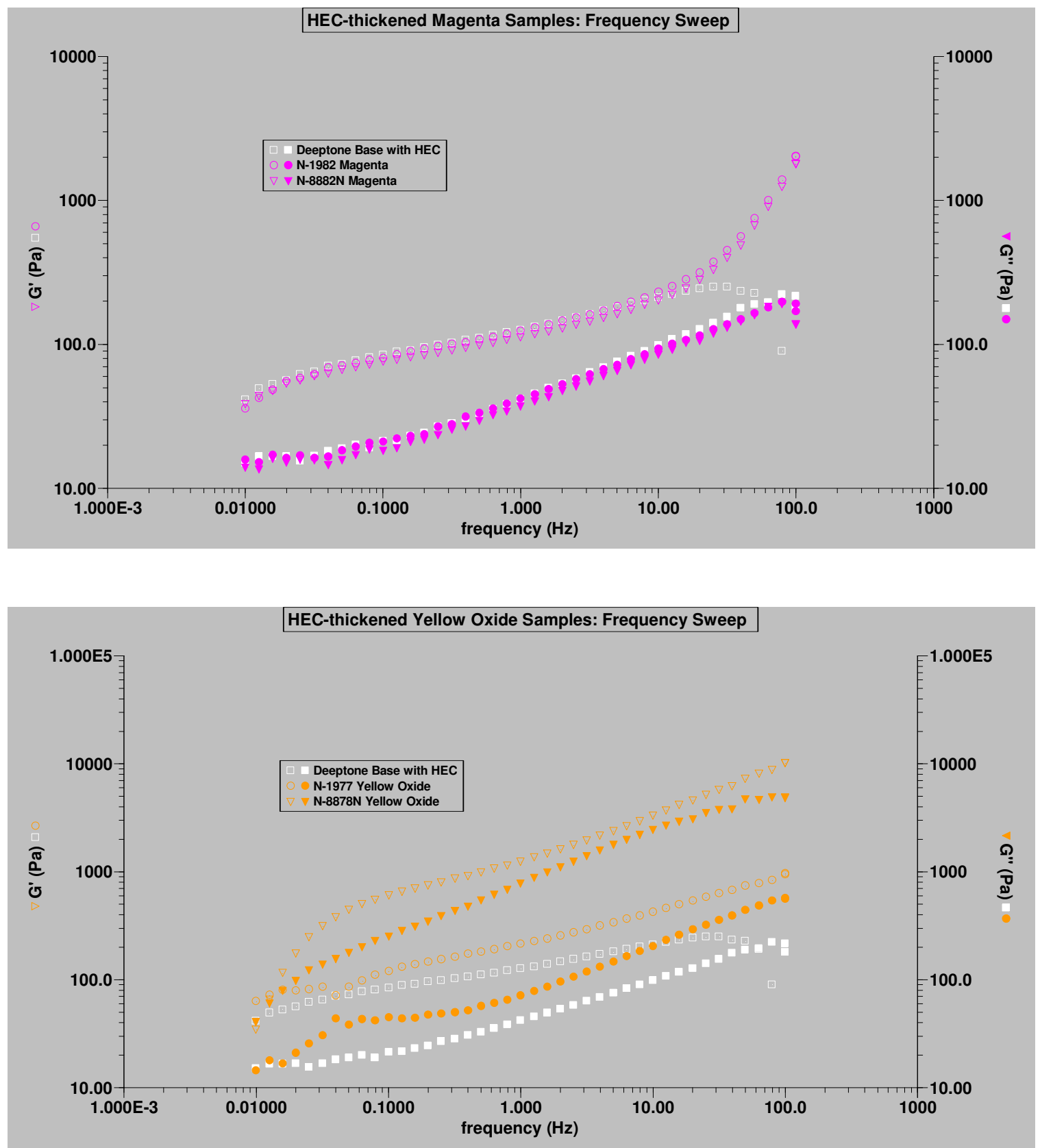

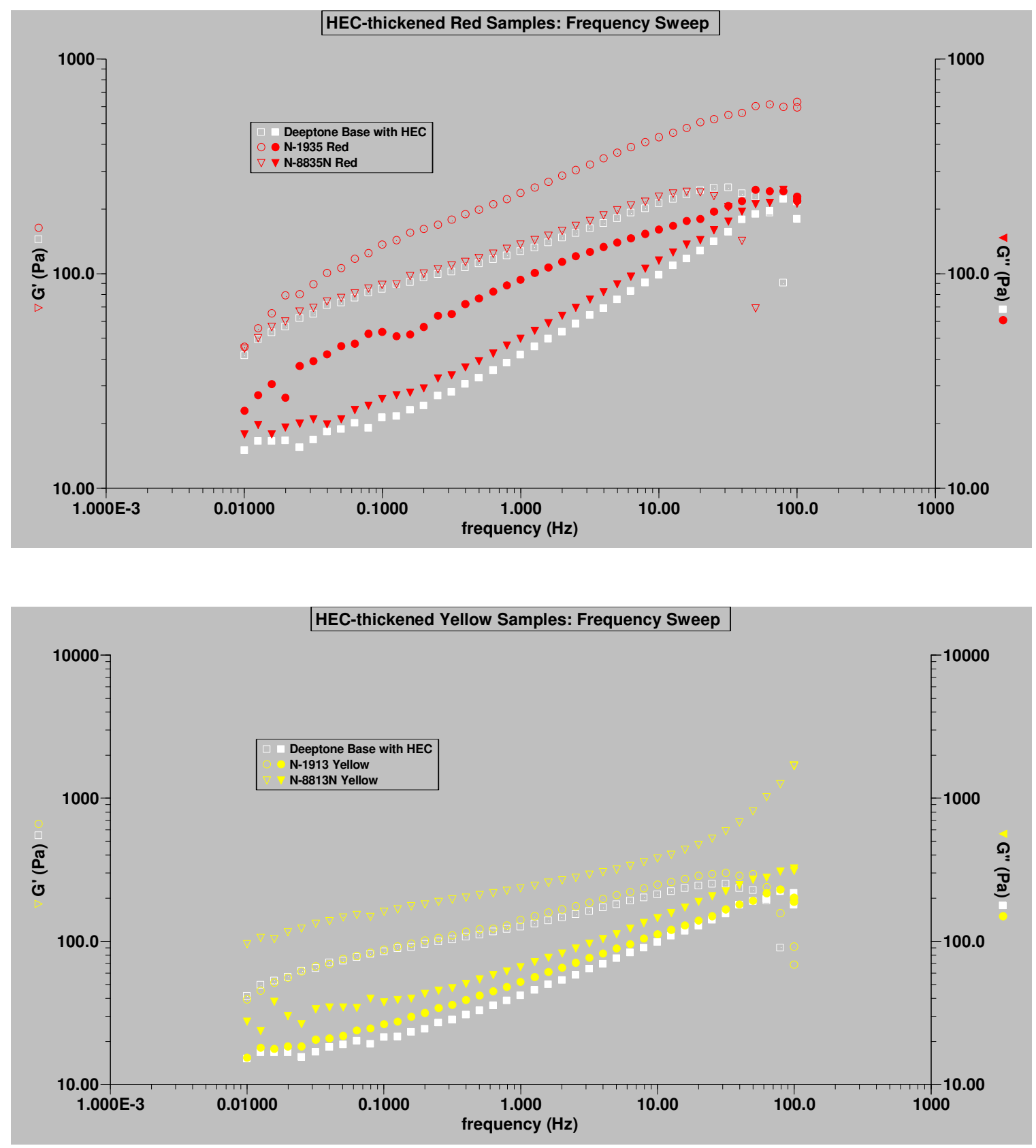

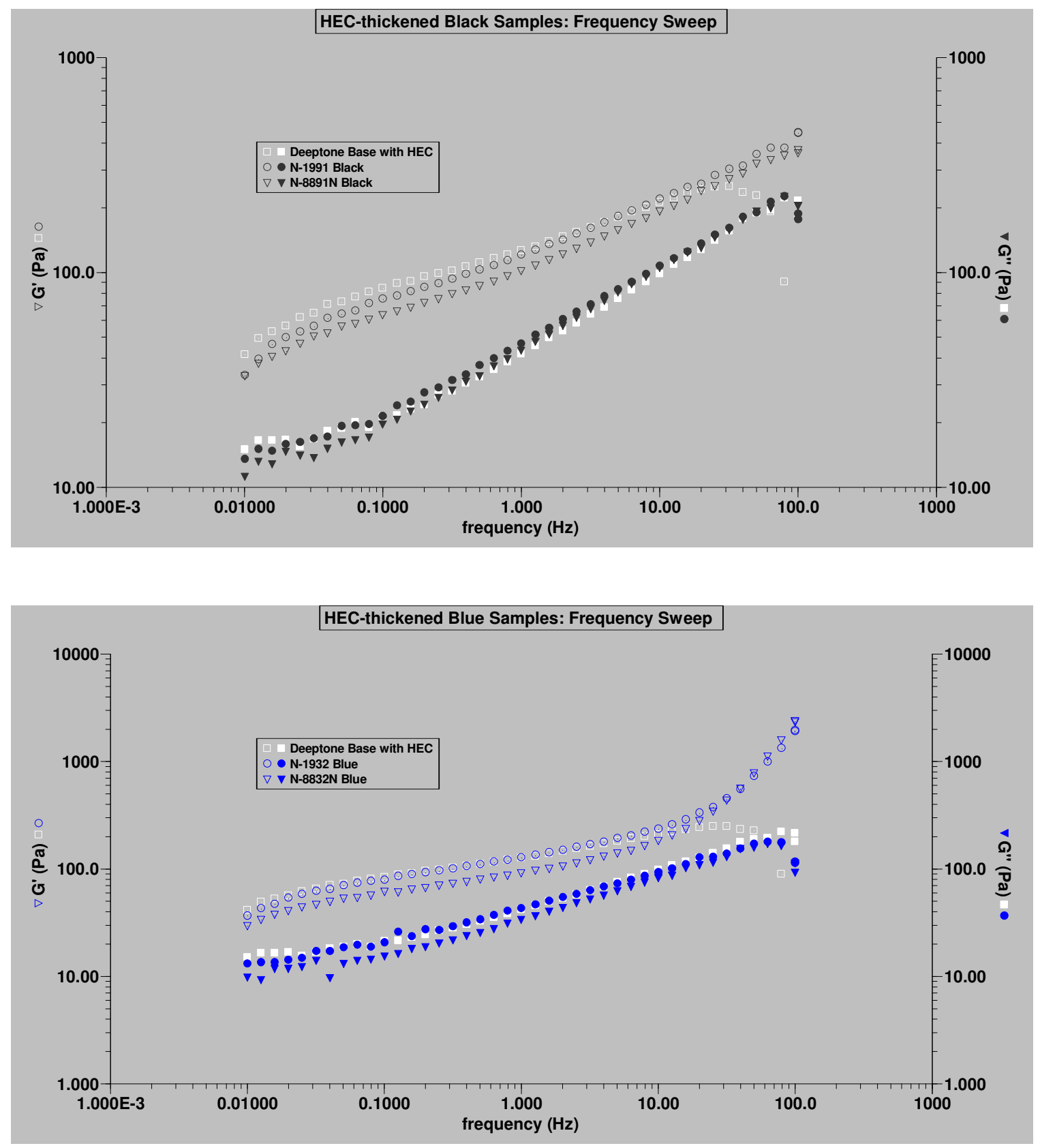

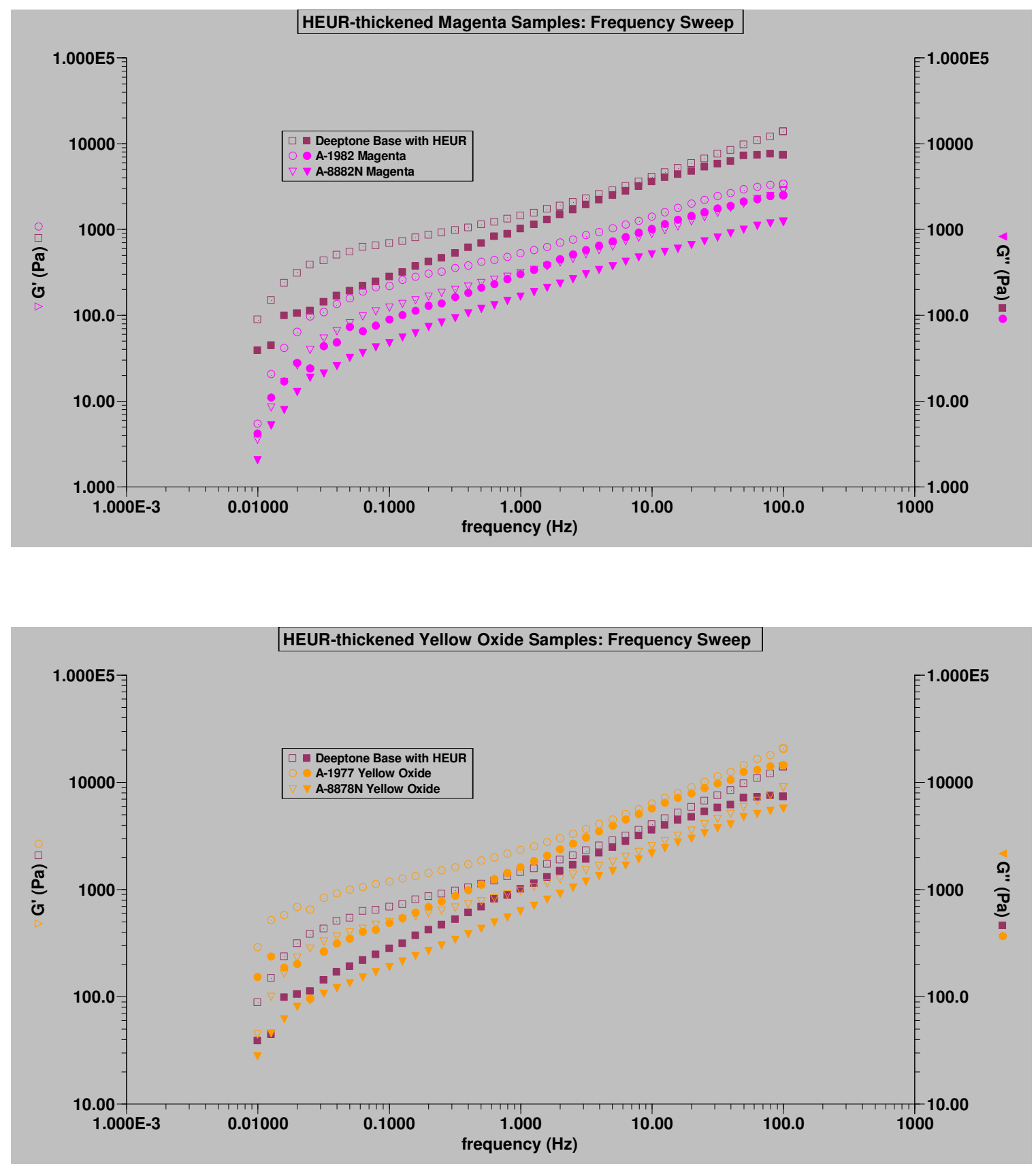

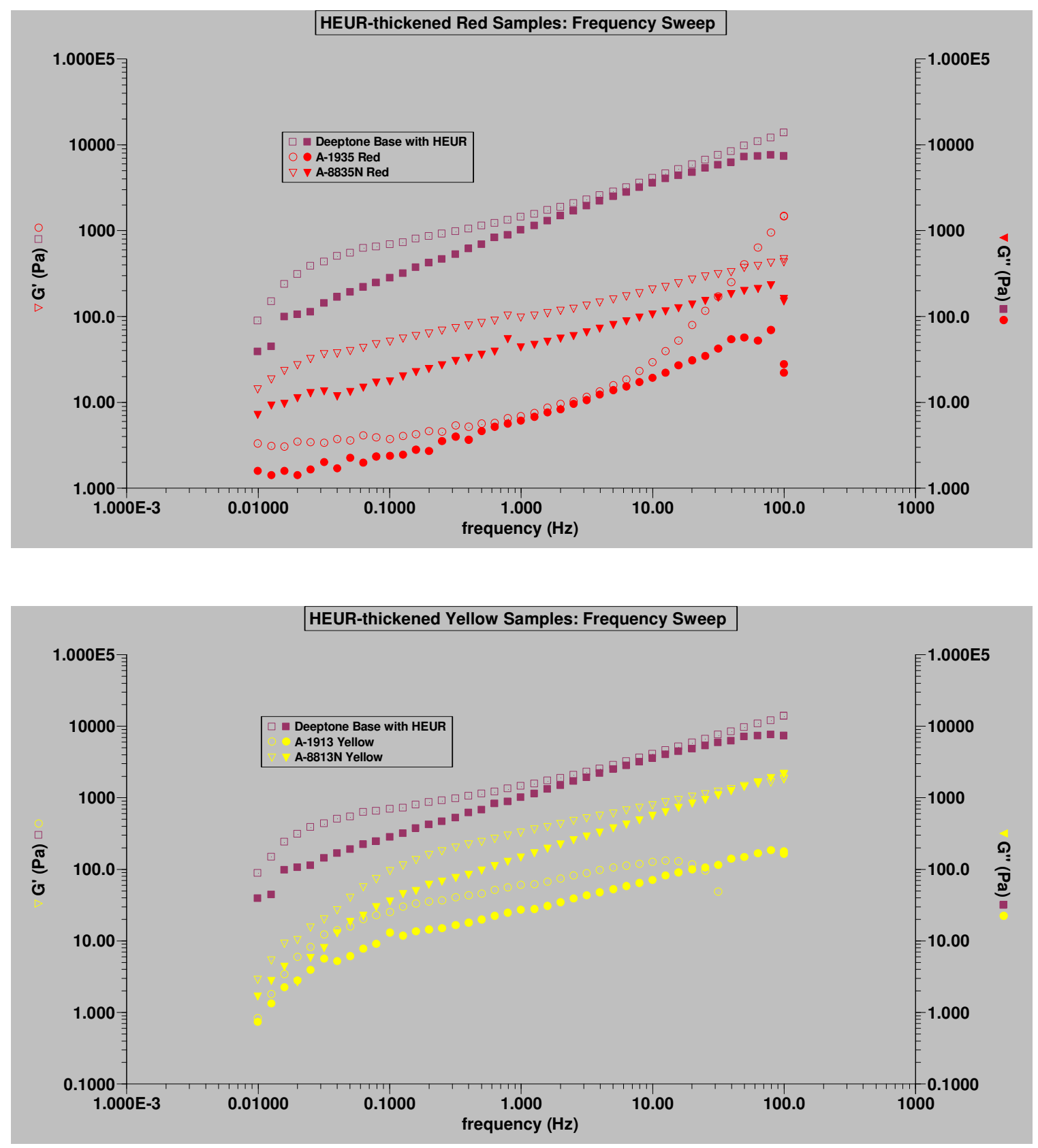

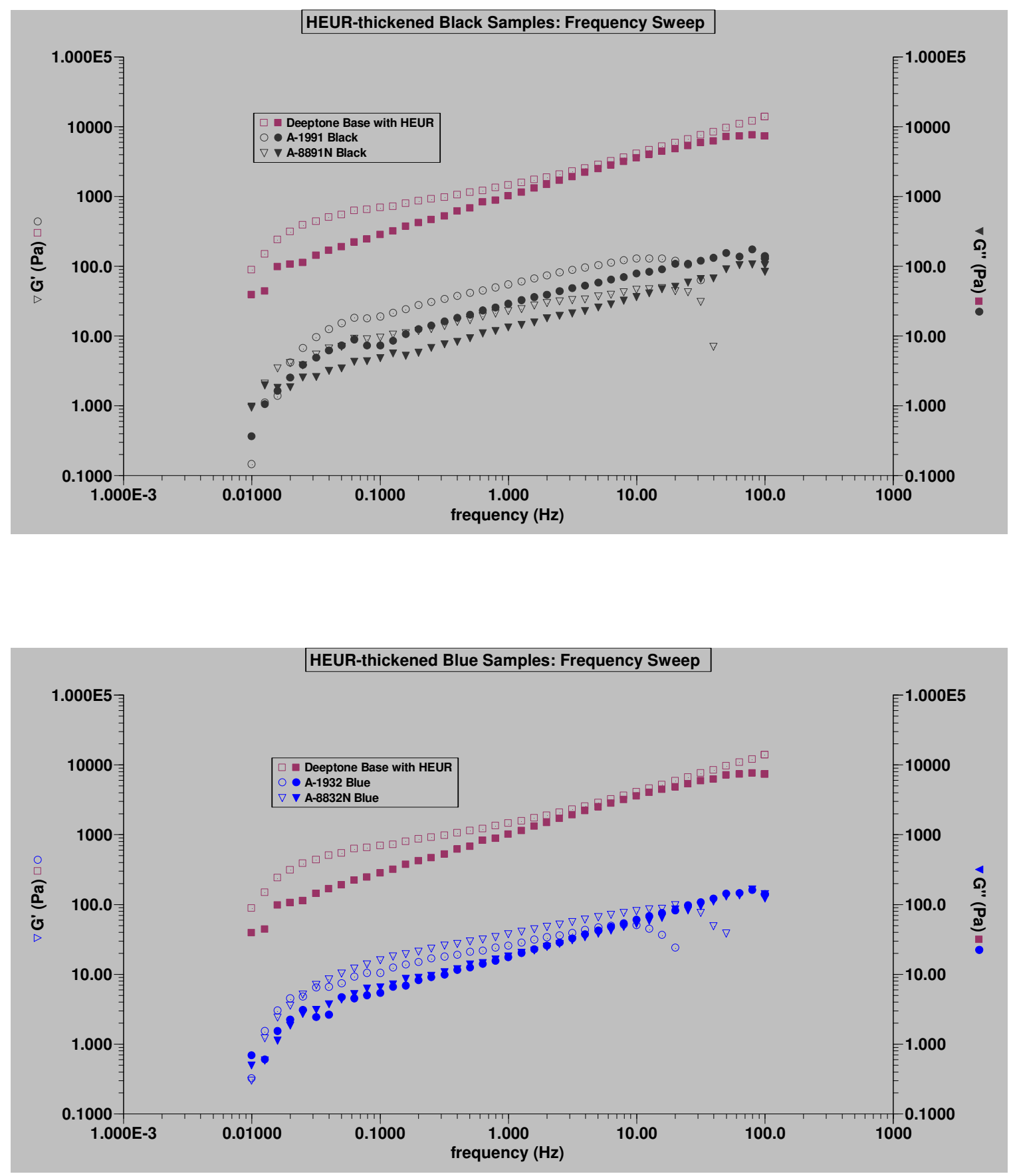


\section{Appendix 13}

Gloss, Tint Strengths, and Contrast Ratios for Pastel Samples

\begin{tabular}{|c|c|c|}
\hline Sample & Tint Strength & Contrast Ratio \\
\hline $\begin{array}{c}\text { Pastel Base with } \\
\text { HEC }\end{array}$ & 99.75995 & 0.99320297 \\
\hline $\begin{array}{c}\text { Pastel Base with } \\
\text { HEUR }\end{array}$ & 99.56901 & 0.993875856 \\
\hline N-1921 Green & 4.103418 & 0.999763761 \\
\hline A-1921 Green & 3.454331 & 0.999266145 \\
\hline N-8821N Green & 4.103362 & 1.004470588 \\
\hline A-8821N Green & 3.731881 & 1.004321729 \\
\hline N-1982 Magenta & 11.52047 & 1.000275406 \\
\hline A-1982 Magenta & 10.80054 & 0.997745209 \\
\hline N-8882N Magenta & 11.79019 & 1.00219901 \\
\hline A-8882N Magenta & 10.35447 & 1.000285633 \\
\hline $\begin{array}{c}\text { N-1977 Yellow } \\
\text { Oxide }\end{array}$ & 1.944938 & 1.000387522 \\
\hline $\begin{array}{c}\text { A-1977 Yellow } \\
\text { Oxide }\end{array}$ & 1.866136 & 1.000393546 \\
\hline N-8878N Yellow & 1.934603 & 1.001536688 \\
\hline A-8878N Yellow & 1.722485 & 1.00235248 \\
\hline N-1935 Red Oxide & 2.380671 & 0.998620055 \\
\hline A-1935 Red Oxide & 2.080304 & 1.009694619 \\
\hline $\begin{array}{l}\text { N-8835N Red } \\
\text { Oxide }\end{array}$ & 2.407932 & 1.000919118 \\
\hline $\begin{array}{l}\text { A-8835N Red } \\
\text { Oxide }\end{array}$ & 2.400016 & 1.011054813 \\
\hline N-1913 Yellow & 2.277169 & 0.993317362 \\
\hline A-1913N Yellow & 2.110607 & 0.992860785 \\
\hline N-8813N Yellow & 2.295555 & 0.996332364 \\
\hline A-8813N Yellow & 2.183737 & 0.99618854 \\
\hline N-1991 Black & 0.836924 & 1.001203369 \\
\hline A-1991 Black & 1.379818 & 1.009302326 \\
\hline N-8891N Black & 1.079744 & 1 \\
\hline A-8891N Black & 1.960975 & 0.997946612 \\
\hline N-1932 Blue & 53.47476 & 1.00125 \\
\hline A-1932 Blue & 51.66443 & 0.998412698 \\
\hline N-8832N Blue & 46.87864 & 1.000672721 \\
\hline A-8832N Blue & 40.34233 & 0.998897059 \\
\hline
\end{tabular}




\section{Appendix 14}

\section{Gloss and Contrast Ratios for Deeptone Samples}

\begin{tabular}{|c|c|c|c|}
\hline Sample & $60^{\circ}$ Gloss & $85^{\circ}$ Gloss & Contrast Ratio \\
\hline Pastel Base with HEC & 68.675 & 90.6 & 0.06112 \\
\hline Pastel Base with HEUR & 70.875 & 94.26 & 0.06053 \\
\hline N-1921 Green & 45.96667 & 75.86667 & 1.05027 \\
\hline A-1921 Green & 54.96667 & 82.5 & 0.919929 \\
\hline N-8821N Green & 48.6 & 76.025 & 0.906646 \\
\hline A-8821N Green & 55.56667 & 82.675 & 0.93554 \\
\hline N-1982 Magenta & 51.86667 & 76.55 & 0.789668 \\
\hline A-1982 Magenta & 56.26667 & 84.5 & 0.78564 \\
\hline N-8882N Magenta & 49.9 & 81.53333 & 0.78941 \\
\hline A-8882N Magenta & 57.23333 & 86.13333 & 0.786683 \\
\hline N-1977 Yellow Oxide & 60.03333 & 85.66 & 0.995698925 \\
\hline A-1977 Yellow Oxide & 65.56667 & 91.83333 & 0.998759305 \\
\hline N-8878N Yellow Oxide & 70 & 86.83333 & 0.996952149 \\
\hline A-8878N Yellow Oxide & 71.4 & 96.575 & 0.99421261 \\
\hline N-1935 Red & 63.76667 & 91.53333 & 1 \\
\hline A-1935 Red & 70.03333 & 96.86667 & 1.010791367 \\
\hline N-8835N Red & 66.43333 & 92 & 0.997601918 \\
\hline A-8835N Red & 71.3 & 95.38 & 1.001650165 \\
\hline N-1913 Yellow & 54.36667 & 85 & 0.7680111 \\
\hline A-1913N Yellow & 58.63333 & 90.3 & .6768713 \\
\hline N-8813N Yellow & 52.8 & 82.85 & .6305473 \\
\hline A-8813N Yellow & 59.1 & 89.3 & .6358455 \\
\hline N-1991 Black & 50.5 & 85.8 & 0.997701149 \\
\hline A-1991 Black & 64.63333 & 96.8 & 0.988151659 \\
\hline N-8891N Black & 57.53333 & 89.03333 & 1.002336449 \\
\hline A-8891N Black & 66.83333 & 95.83333 & 1 \\
\hline N-1932 Blue & 43.46667 & 77.25 & 0.964844 \\
\hline A-1932 Blue & 50.13333 & 84.33333 & 0.924901 \\
\hline N-8832N Blue & 55.33333 & 84.83333 & 0.957265 \\
\hline A-8832N Blue & 69.16667 & 95.5 & 0.932203 \\
\hline
\end{tabular}




\section{Appendix 15}

Viscosity Profiles of DOW and Turk Samples Arranged by Color
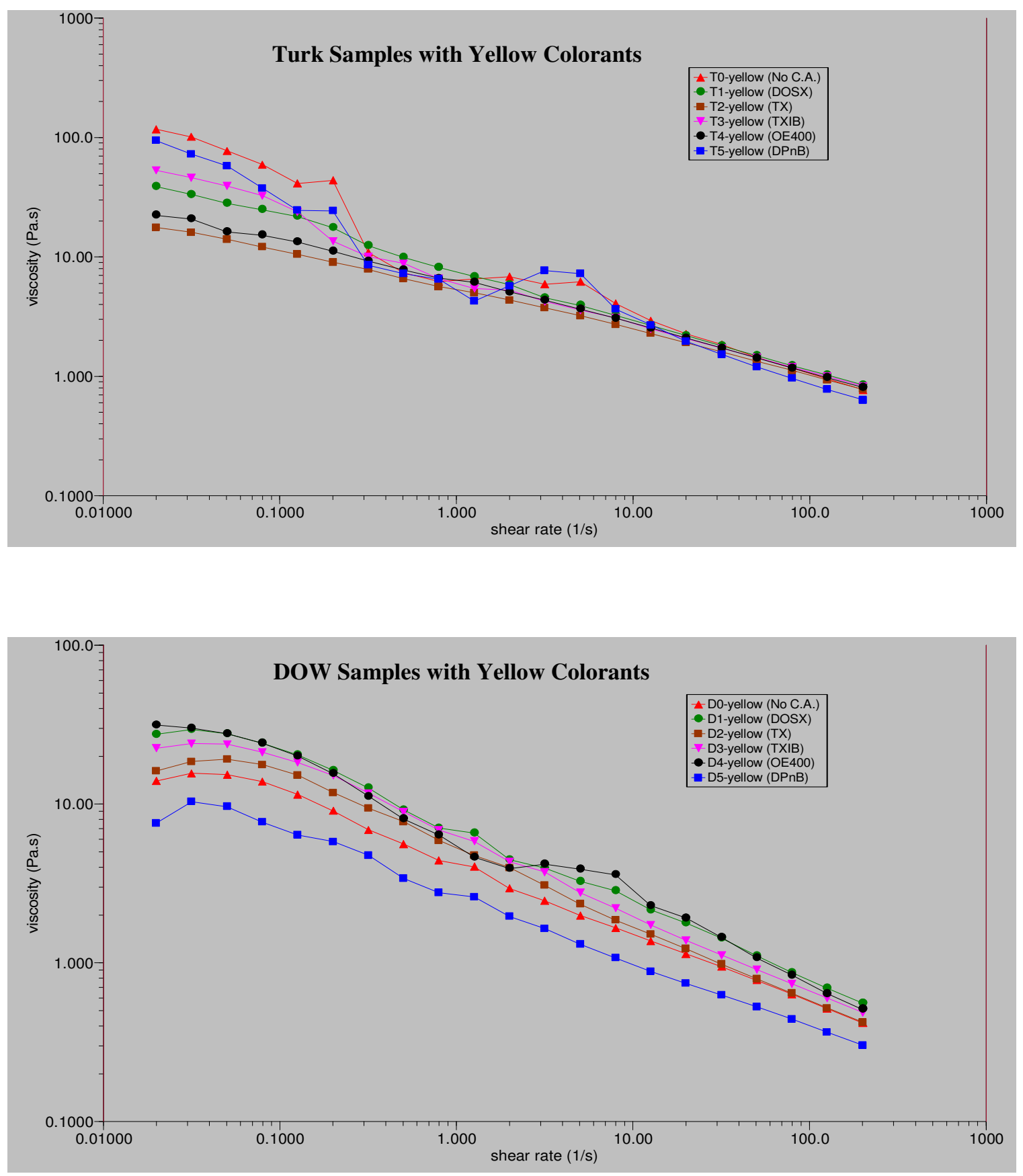

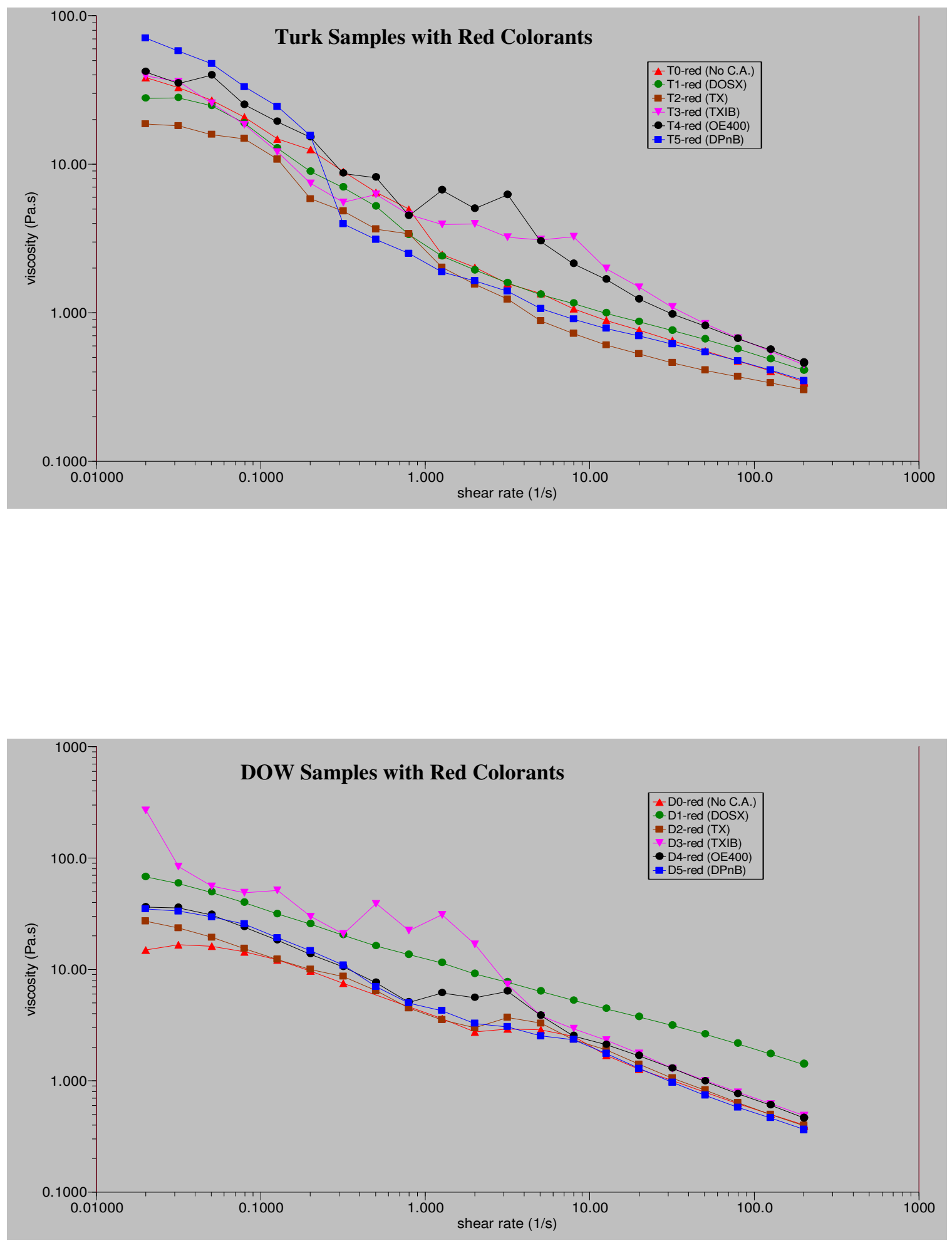


\section{Appendix 16}

Viscosity Profiles of DOW and Turk Samples Arranged by Coalescing Aid
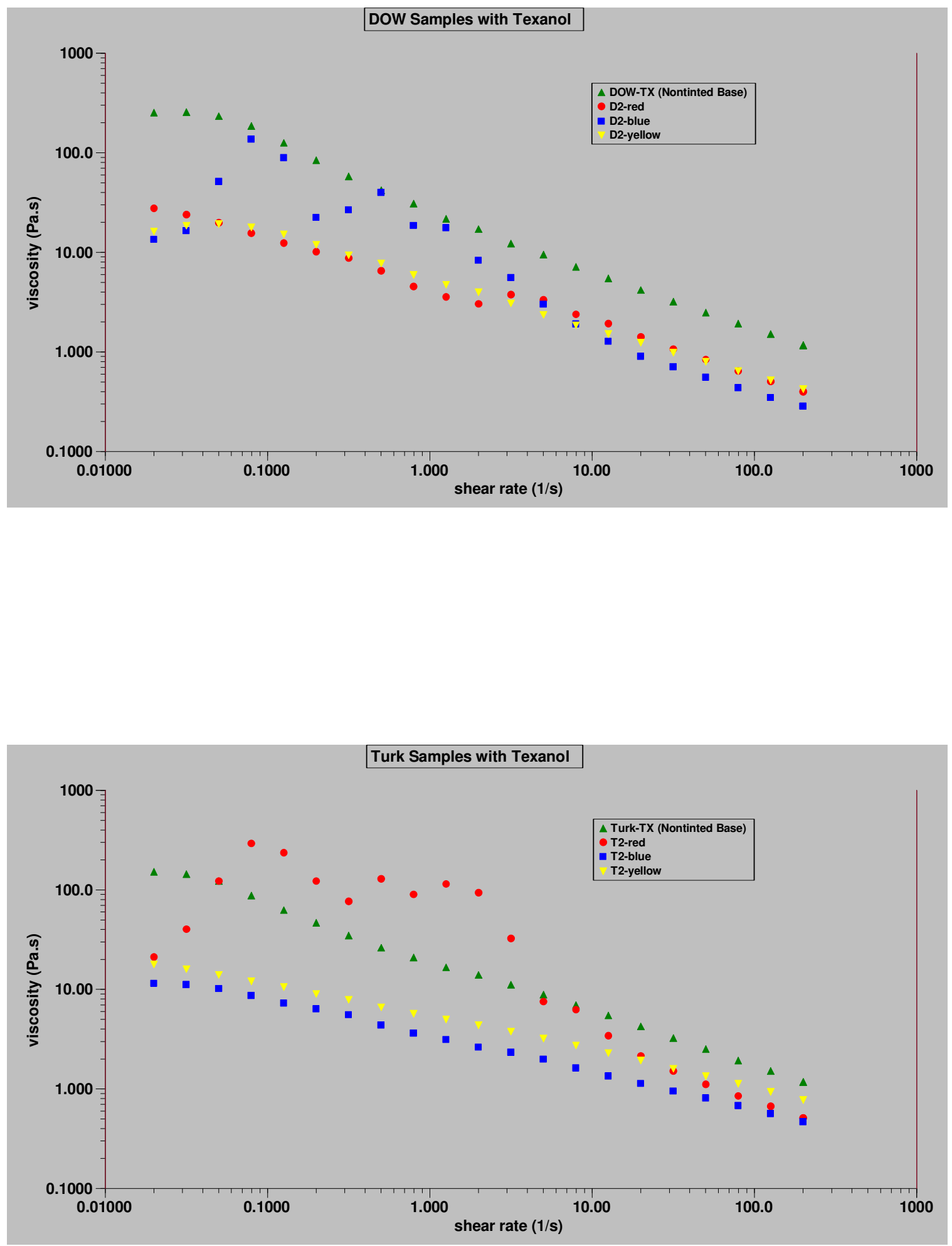

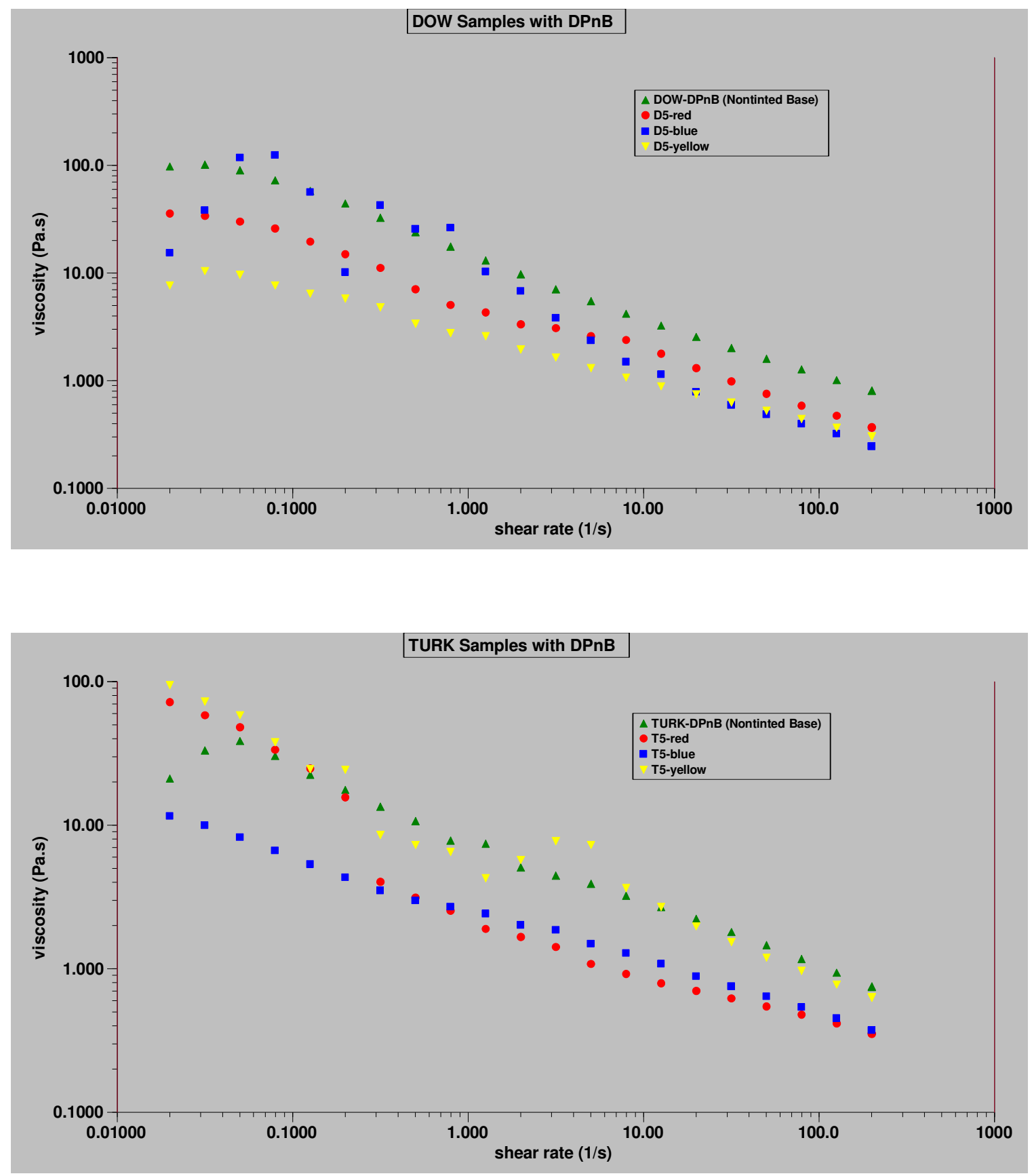

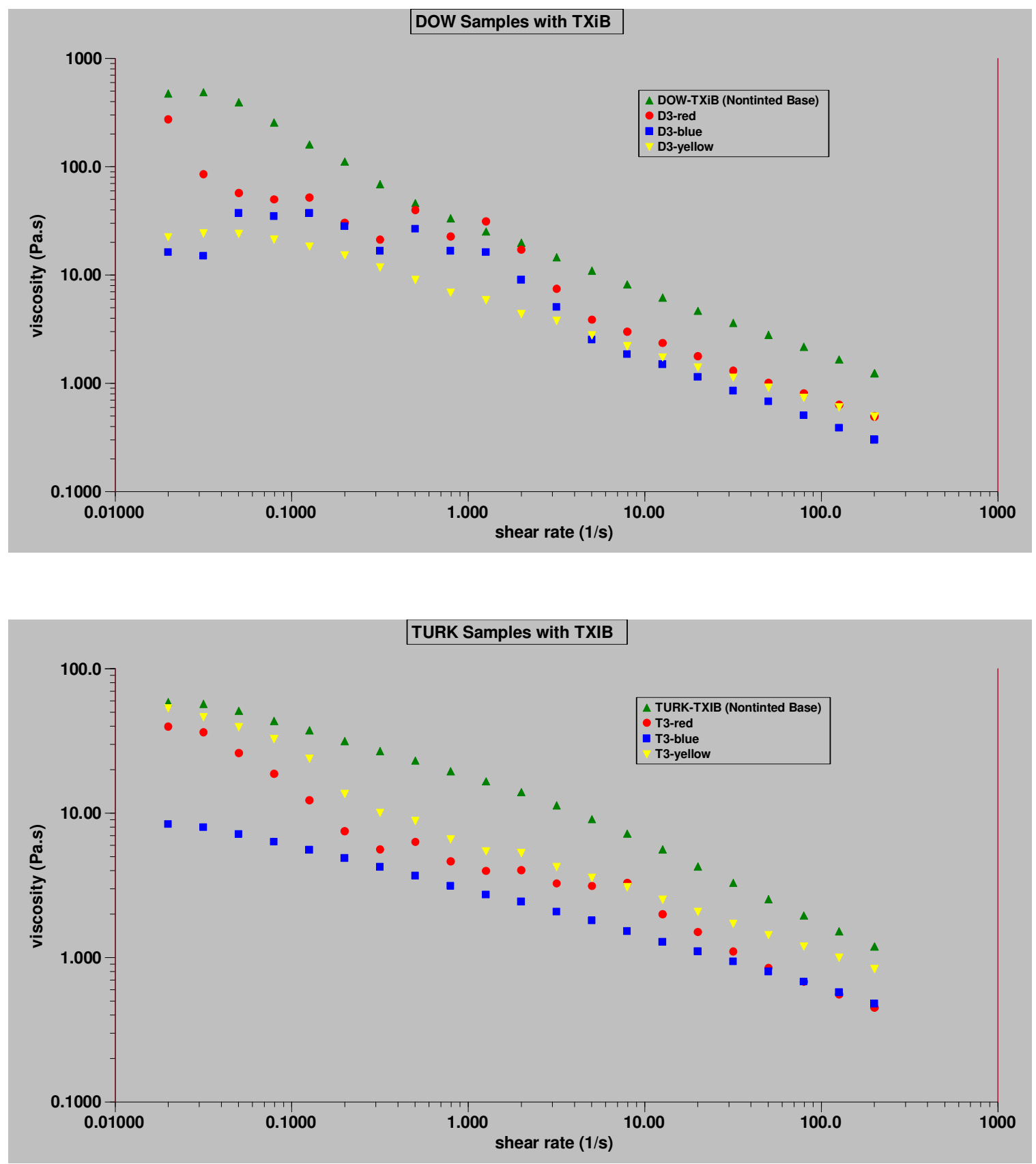

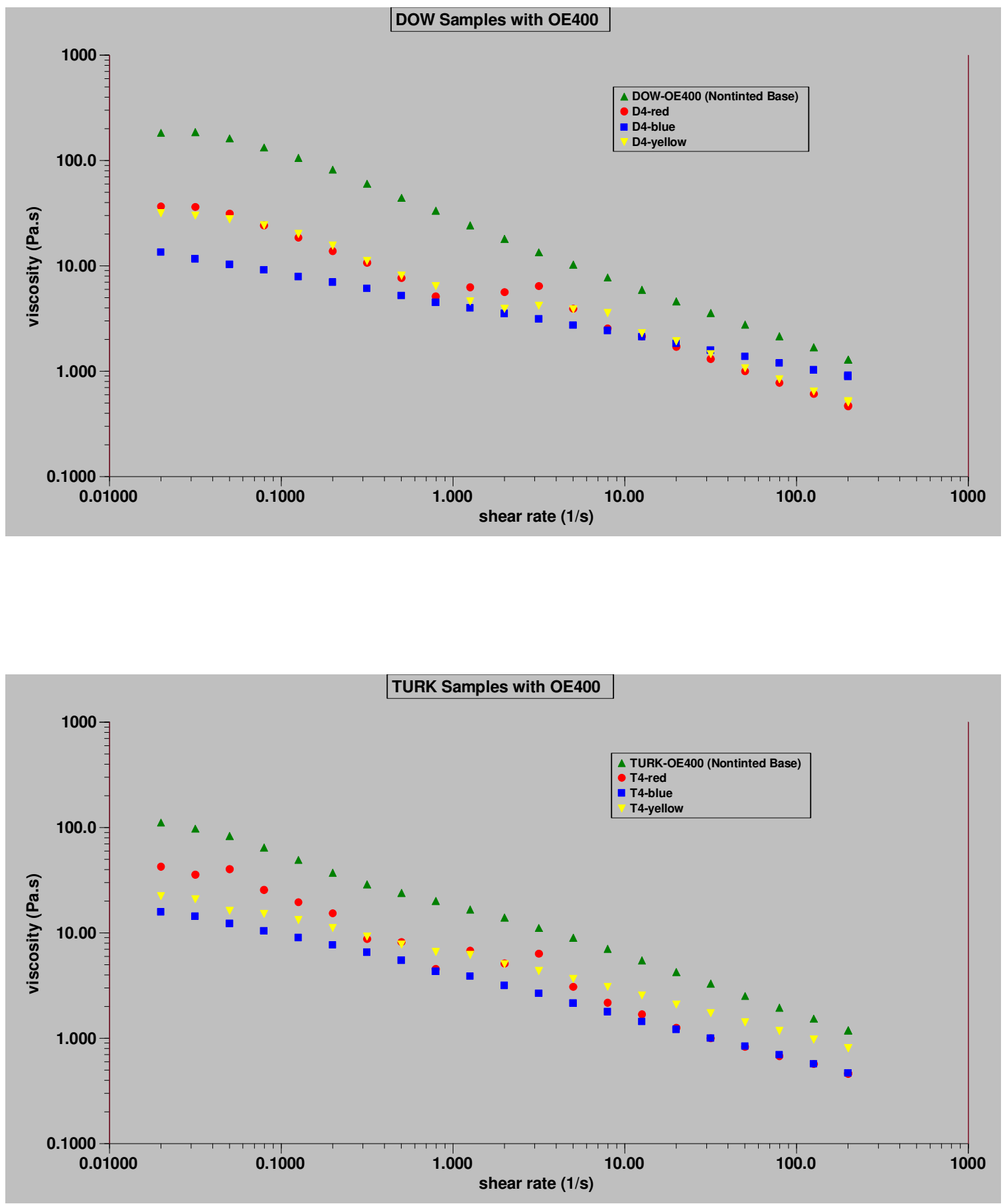